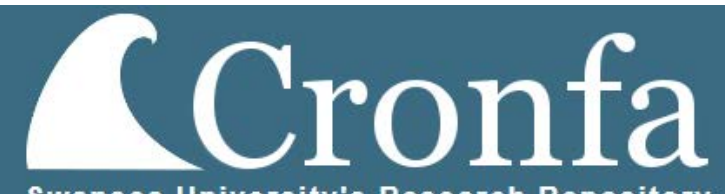

Swansea University's Research Repository

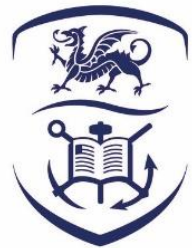

Swansea University

Prifysgol Abertawe

\title{
Opto-Electrical Interactions in Next Generation Semiconductor Thin Films and Devices
}

Robin Kerremans

A thesis submitted for the degree of Doctor of Philosophy at Swansea University

Faculty of Science and Engineering

Department of Physics

2021

Copyright: The author, Robin Kerremans, 2021. 


\section{Acknowledgements}

I would like to thank my two supervisors, Paul Meredith and Ardalan Armin, for their continued support, their enthusiasm, and their guidance in the creation of this thesis. None of this would have been possible without them.

I also want to thank all the members of our group, for their willingness to always extend a helping hand and for all their work together with me. The projects described in this thesis wouldn't have been nearly as enjoyable without such a great group to fall back on.

Some more deep gratitude goes out to the rock-climbing gyms in Swansea and the beautiful Welsh cliffs for providing me with the distractions of rock climbing, and to the De La Beche Chess club for taking up some of my spare brain capacity.

Finally, I'm grateful to all my international friends in the physics department and all my local Welsh friends, not in the least my housemates, for helping me get through the harder times and most importantly for making these past three years as unforgettable as they were.

Thank you, it's been a blast. 


\section{Definition of general constants, variables and common materials}

\begin{tabular}{|c|c|c|c|}
\hline symbol & name & value & units \\
\hline \multicolumn{4}{|l|}{ Constants } \\
\hline$h$ & Planck's constant & $6.63 \mathrm{E}-34$ & $\mathrm{~m}^{2} \mathrm{~kg} \mathrm{~s}^{-1}$ \\
\hline c & velocity of light & $3 \mathrm{E} 8$ & $\mathrm{~m} \mathrm{~s}^{-1}$ \\
\hline$k$ & Boltzmann constant & $1.38 \mathrm{E}-23$ & $\mathrm{~m}^{2} \mathrm{~kg} \mathrm{~s}^{-2} \mathrm{~K}^{-1}$ \\
\hline$q$ & elementary charge of an electron/hole & $1.6 \mathrm{E}-19$ & coulombs \\
\hline$\varepsilon_{0}$ & permittivity of vacuum & $8.85 \mathrm{E}-12$ & $m^{-3} k^{-1} s^{4} A^{2}$ \\
\hline$\mu_{0}$ & permeability of vacuum & $1.26 \mathrm{E}-6$ & $m \mathrm{~kg} \mathrm{~s}^{-2} A^{-2}$ \\
\hline$e$ & Euler's constant & 2.72 & \\
\hline$\pi$ & $\mathrm{Pi}$ & 3.14 & \\
\hline \multicolumn{4}{|l|}{$\begin{array}{l}\text { Common } \\
\text { variables }\end{array}$} \\
\hline$T$ & temperature & & ${ }^{\circ} \mathrm{K}$ \\
\hline$v / f$ & frequency & & $\mathrm{s}^{-1}(\mathrm{~Hz})$ \\
\hline$J$ & current density & & $\mathrm{mA} \mathrm{cm}{ }^{-2}$ \\
\hline$V$ & voltage & & $\mathrm{V}$ \\
\hline EQE & external quantum efficiency & & arbitrary units (\%) \\
\hline IQE & internal quantum efficiency & & arbitrary units (\%) \\
\hline$\varepsilon$ & Dielectric constant / relative permittivity & & $\mathrm{F} \mathrm{m}^{-1}$ \\
\hline $\begin{array}{l}\text { Common } \\
\text { materials }\end{array}$ & Full name & & \\
\hline PCDTBT & $\begin{array}{l}\text { Poly[N-9'-heptadecanyl-2,7-carbazole-alt-5,5- } \\
\left(4^{\prime}, 7^{\prime} \text {-di-2-thienyl-2',1',3'-benzothiadiazole)] }\right.\end{array}$ & & \\
\hline $\mathrm{PC}_{61} /{ }_{71} \mathrm{BM}$ & Phenyl-C61/71-butyric acid methyl ester & & \\
\hline PDINO & $\begin{array}{c}\text { 2,9-Bis[3- } \\
\text { (dimethyloxidoamino)propyl]anthra[2,1,9- } \\
\text { def:6,5,10-d'e'f']diisoquinoline-1,3,8,10(2H,9H)- } \\
\text { tetrone }\end{array}$ & & \\
\hline ITO & indium tin oxide & & \\
\hline PEDOT:PSS & $\begin{array}{l}\text { poly(3,4-ethylenedioxythiophene) polystyrene } \\
\text { sulfonate }\end{array}$ & & \\
\hline FTO & Fluoride tin oxide & & \\
\hline MaPbl3 & $\begin{array}{c}\text { Methylammonium Lead lodide (a type of } \\
\text { perovskite) }\end{array}$ & & \\
\hline Y6 & $\begin{array}{l}\text { 2,2'-((2Z,2'Z)-((12,13-bis(2-ethylhexyl)-3,9- } \\
\text { diundecyl-12,13-dihydro-[1,2,5]thiadiazolo[3,4- } \\
\left.\text { e]thieno[2",3'": } 4^{\prime}, 5^{\prime}\right] \text { thieno[2',3':4,5]pyrrolo[3,2 } \\
\text {-g]thieno[2',3':4,5]thieno[3,2-b]indole-2,10- } \\
\text { diyl)bis(methanylylidene))bis(5,6-difluoro-3- }\end{array}$ & & \\
\hline
\end{tabular}




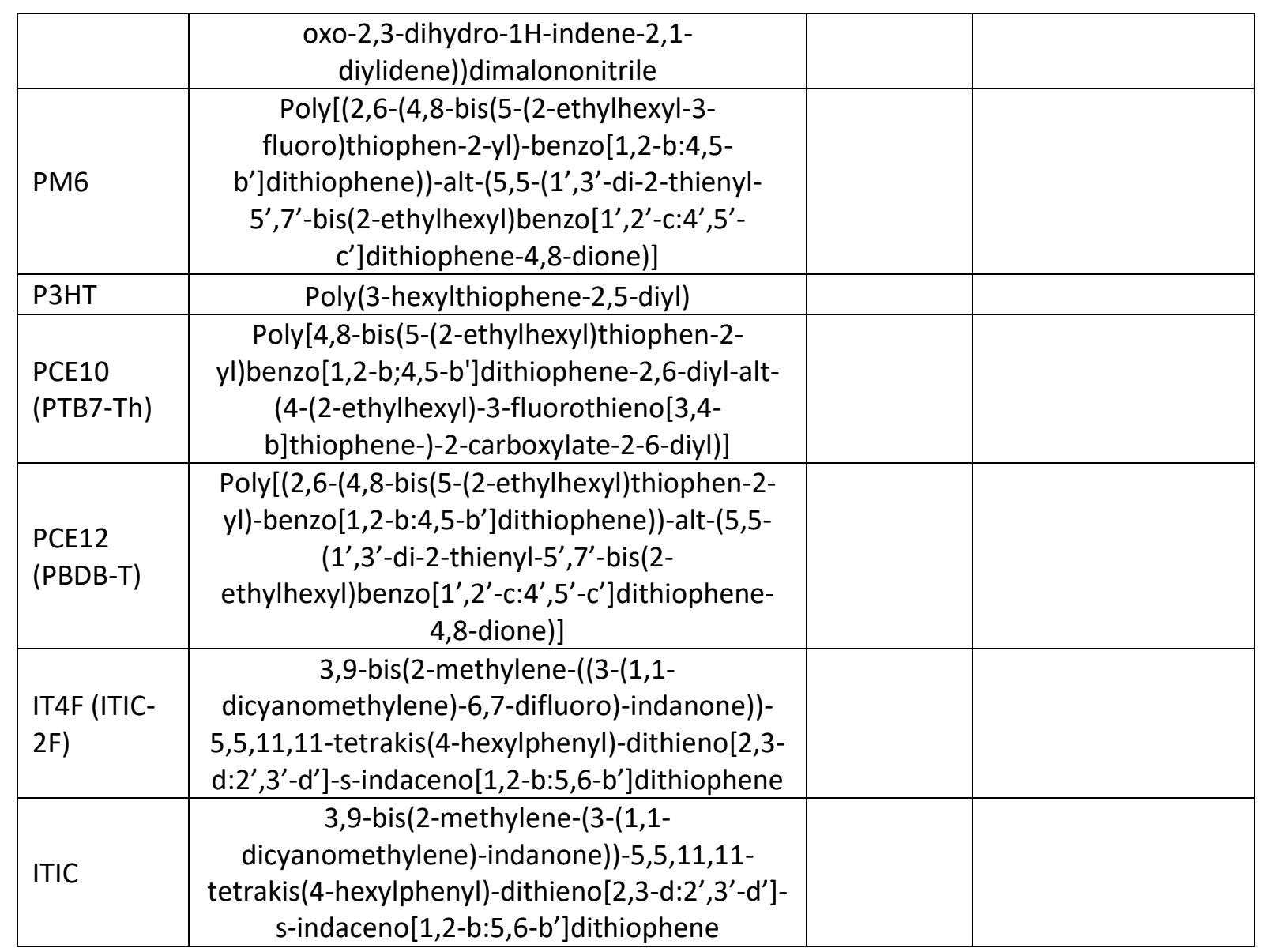




\section{Declarations}

This work was funded by the Welsh Government's Sêr Cymru II Program (Sustainable Advanced Materials) through the European Regional Development Fund and Welsh European Funding Office. The author is recipient of the EPSRC DTP postgraduate award.

This work has not previously been accepted in substance for any degree and is not being concurrently submitted for any degree.

Signed:

Date: $31 / 03 / 2021$

\section{Statement 1}

This thesis is the result of my own investigations, except where otherwise stated. Where correction services have been used, the extent and nature of the correction is clearly marked in a footnote(s). Other sources are acknowledged by footnotes giving explicit references. A bibliography is appended.

Signed:

Date: $31 / 03 / 2021$

Statement 2

I hereby give consent for my thesis, if accepted, to be available for photocopying and for inter-library loan, and for the title and summary to be made available to outside organizations.

Signed:

Date: $31 / 03 / 2021$ 


\section{List of Figures}

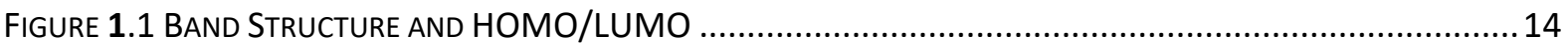

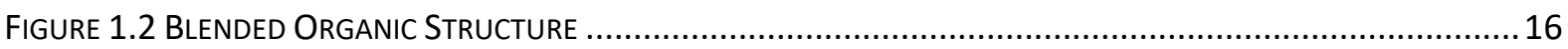

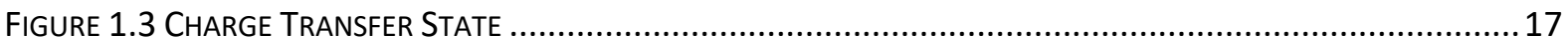

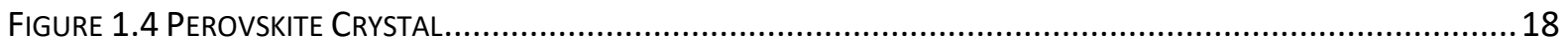

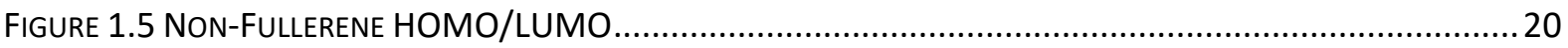

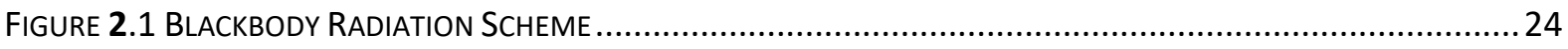

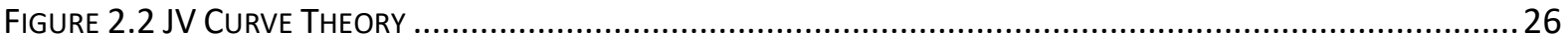

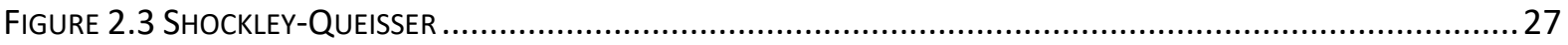

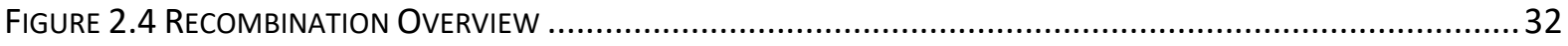

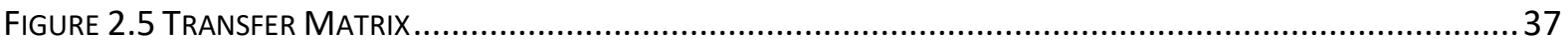

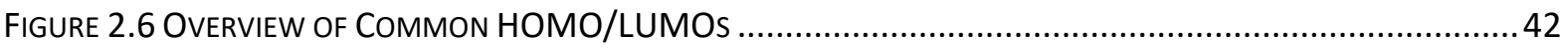

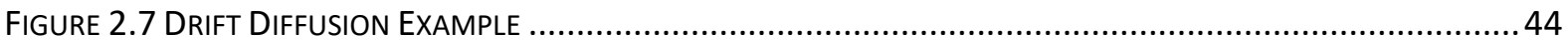

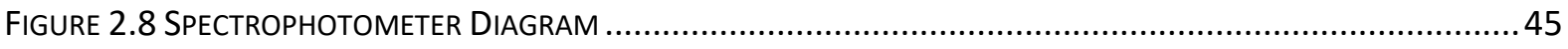

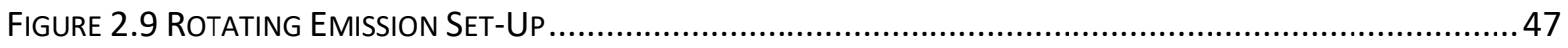

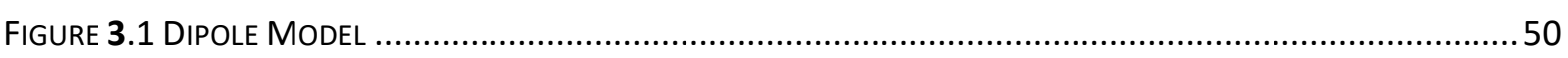

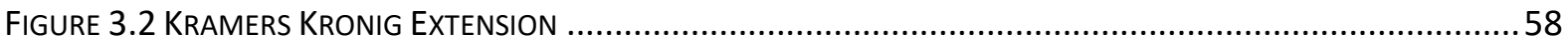

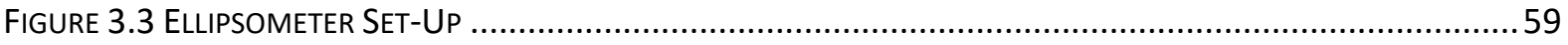

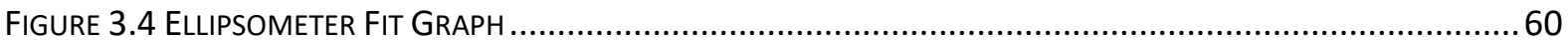

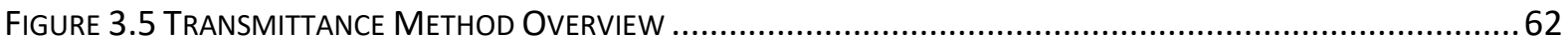

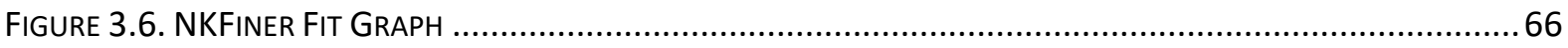

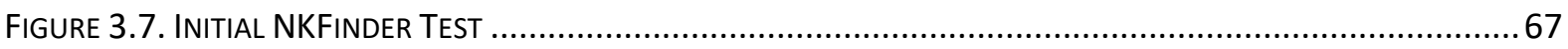

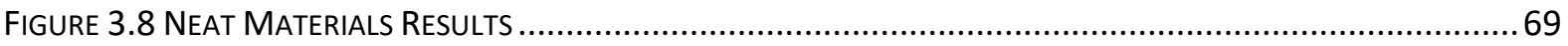

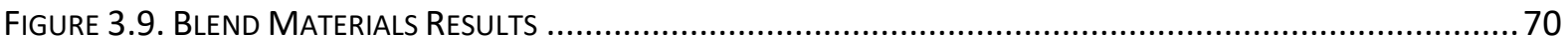

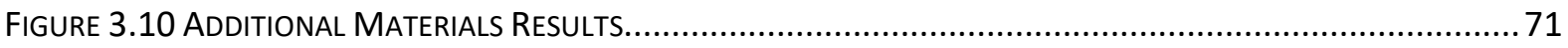

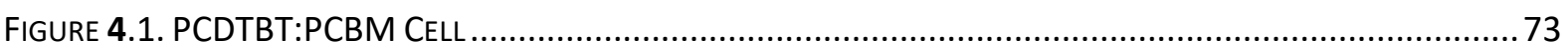

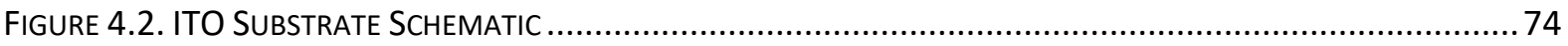

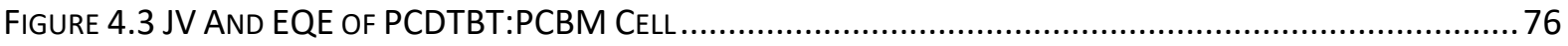

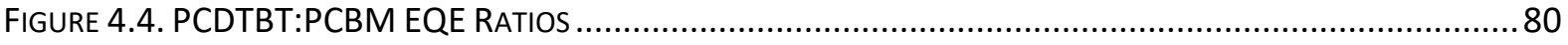

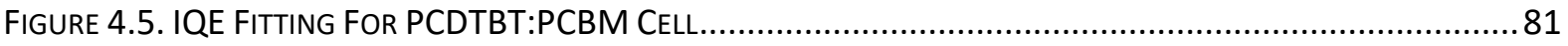




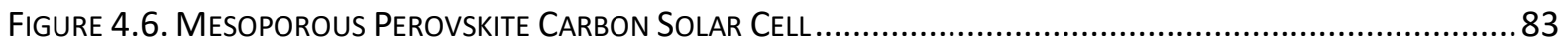

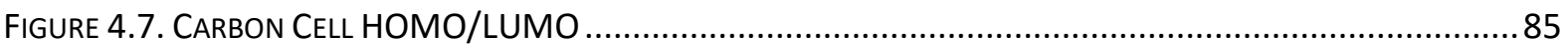

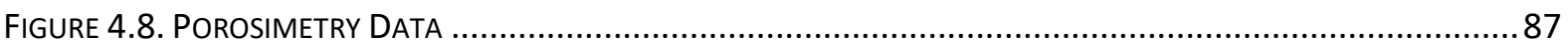

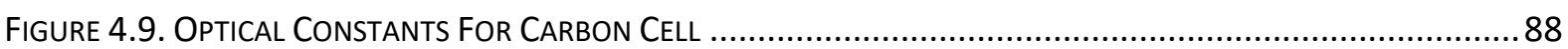

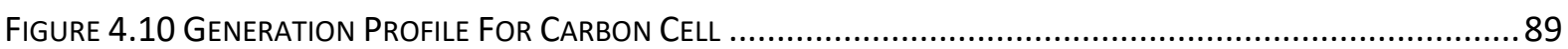

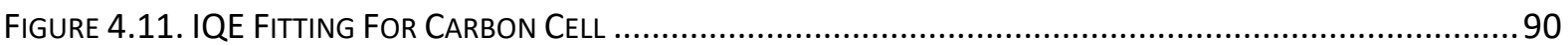

FIGURE 4.12. Simulated THICKNESS DePENDENCE For CARBon CELL JV....................................................... 92

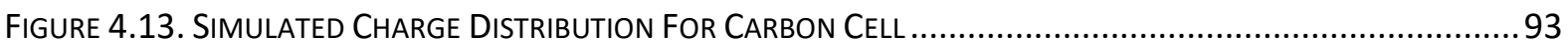

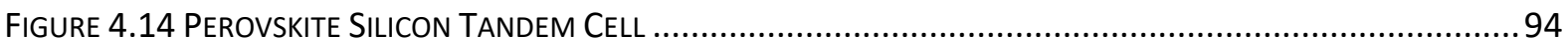

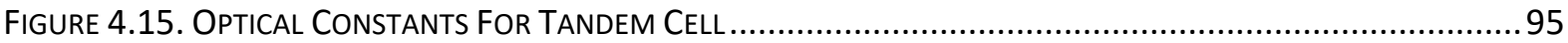

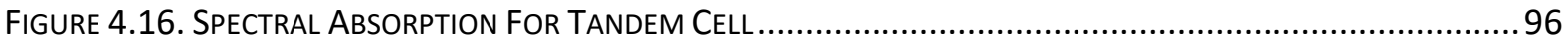

FIGURE 4.17 GeNERATION PROFILE FOR TANDEM CELL............................................................................ 97

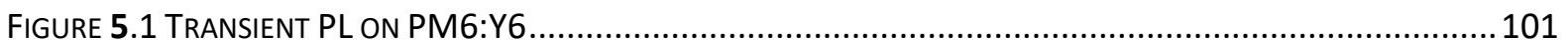

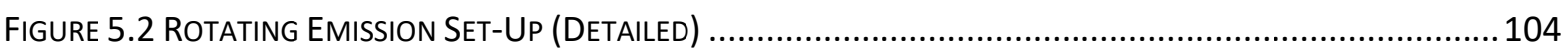

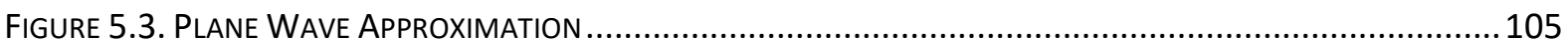

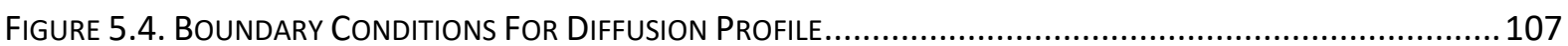

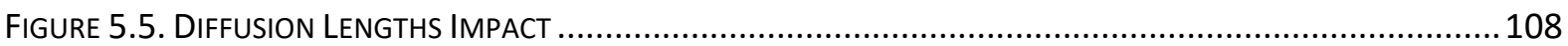

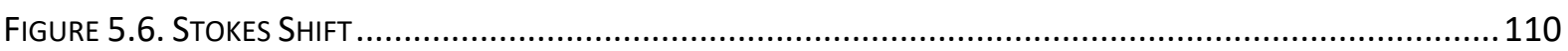

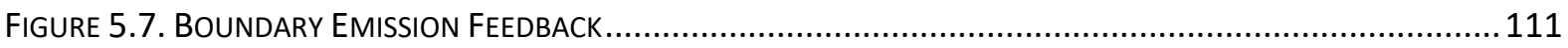

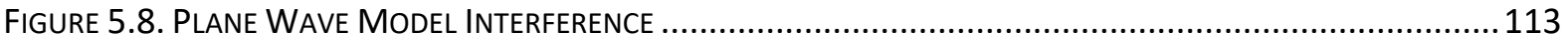

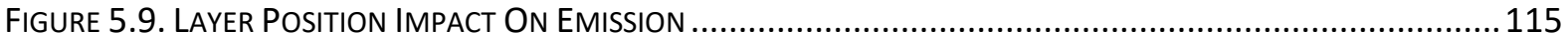

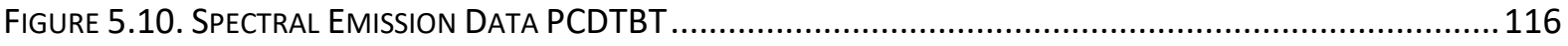

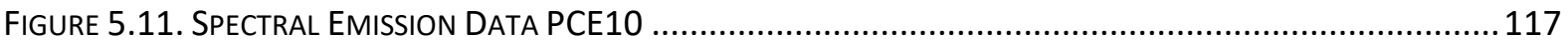

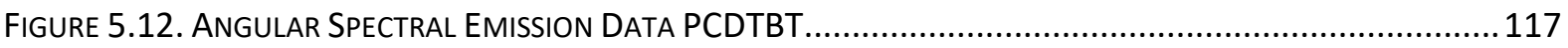

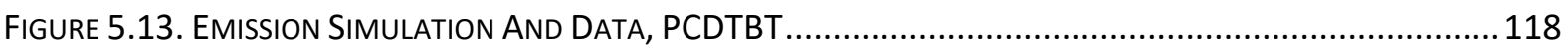

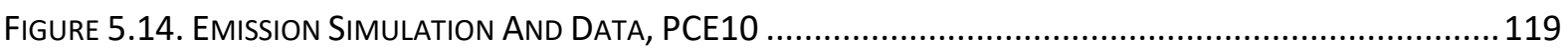

Figure 5.15. Averaged Emission Data, Measured And CorreCted..................................................120 


\section{List of Flowcharts}

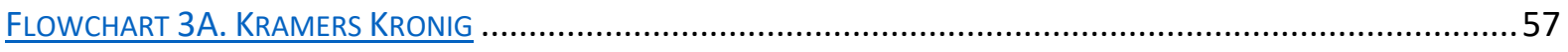

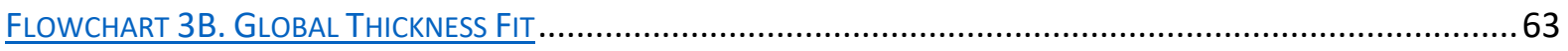

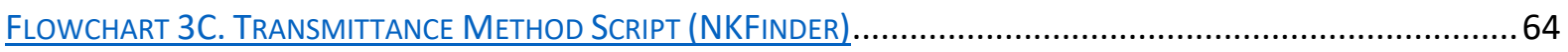

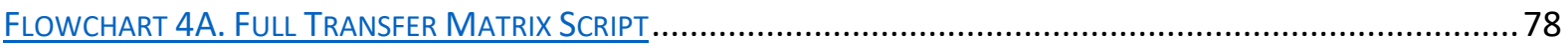

\section{List of Tables}

TABLE 5A. Details on the Marcus Fit of Averaged Emission in 5.15. 


\section{Full Table of Contents}

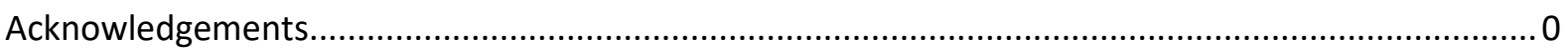

Definition of general constants, variables and common materials .................................................... 3

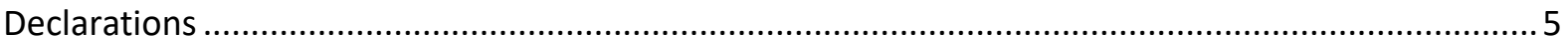

\section{ABSTRACT}

\section{INTRODUCTION}

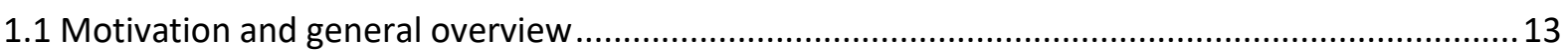

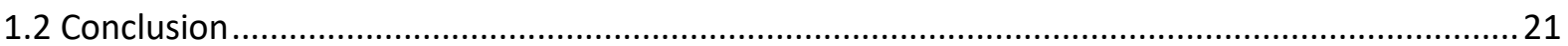

II. THEORY OF OPTO-ELECTRICAL DEVICES

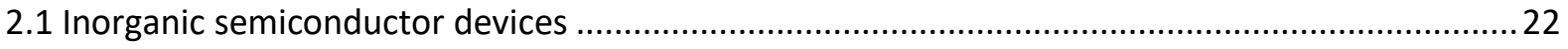

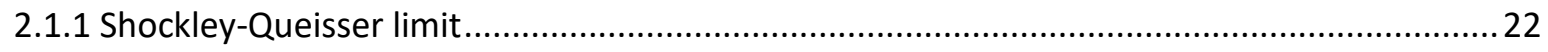

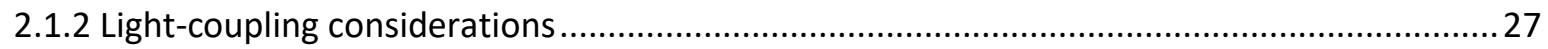

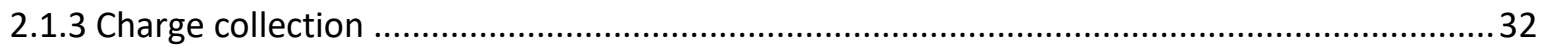

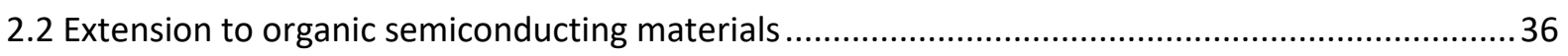

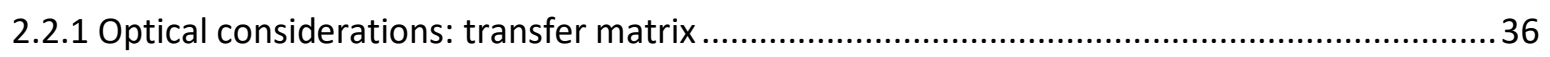

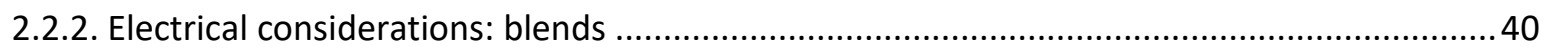

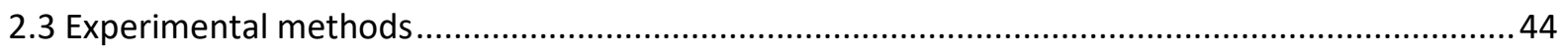

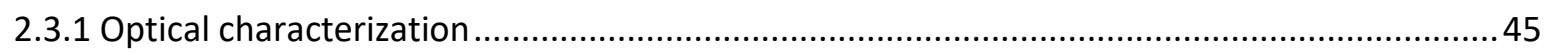

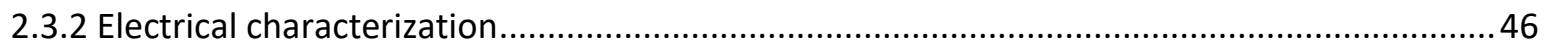

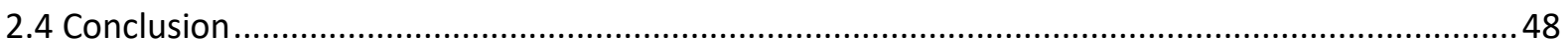

\section{DETERMINATION OF OPTICAL CONSTANTS}

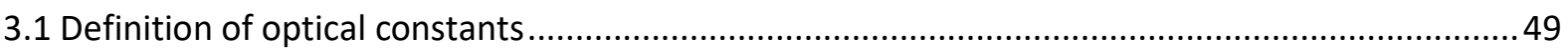

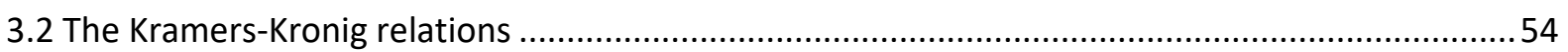

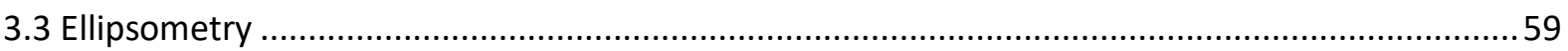

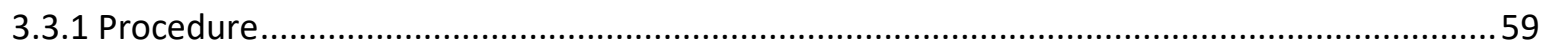

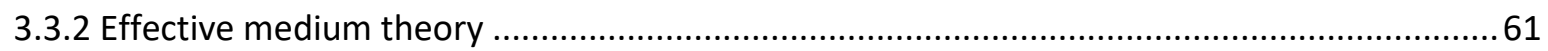

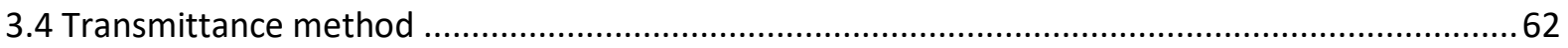

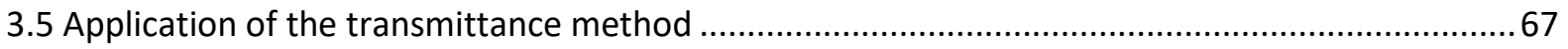

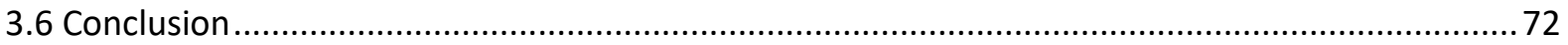

IV. APPLICATION TO THIRD-GENERATION DEVICES IN PHOTOVOLTAICS

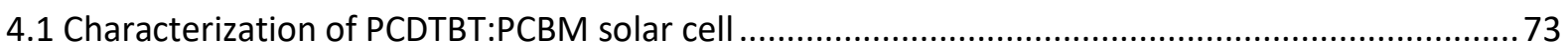

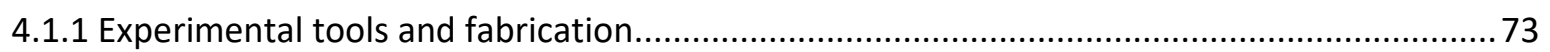

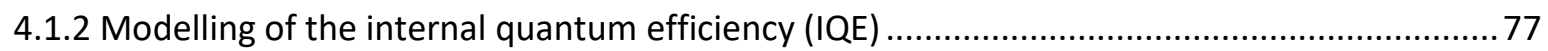




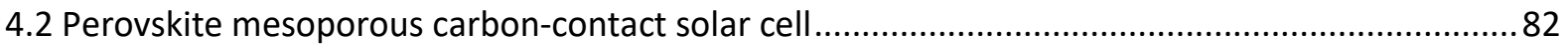

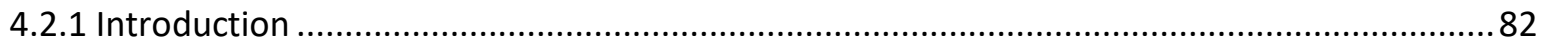

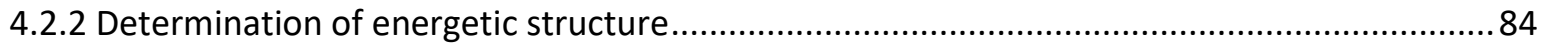

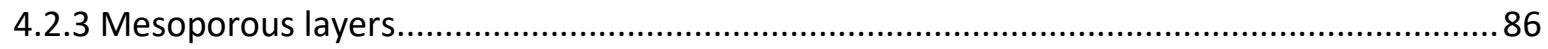

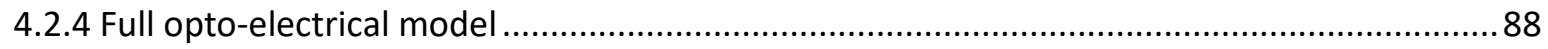

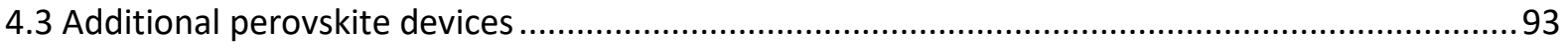

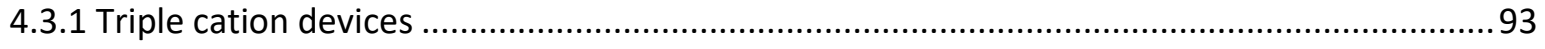

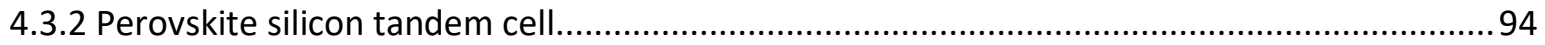

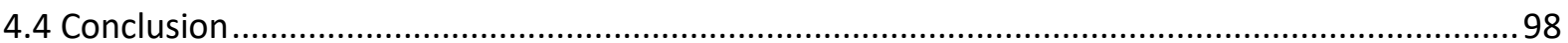

\section{OUT-COUPLING MODEL FOR ACTIVE LAYER PHOTOLUMINESCENCE}

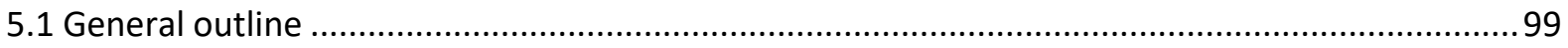

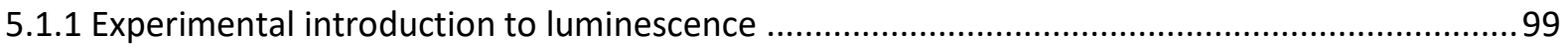

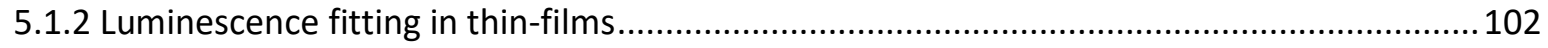

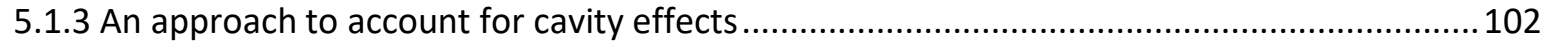

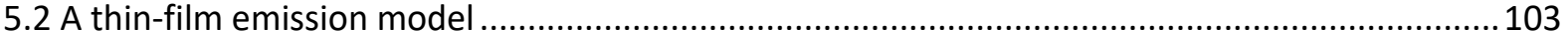

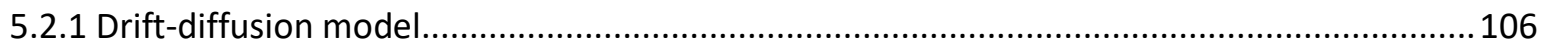

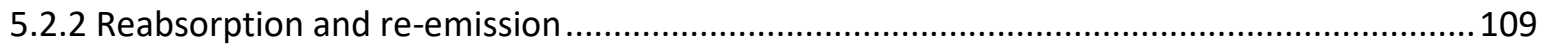

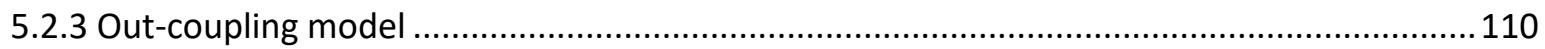

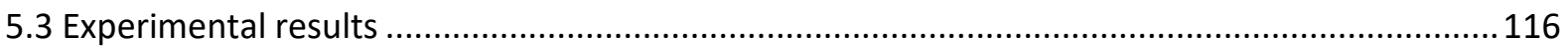

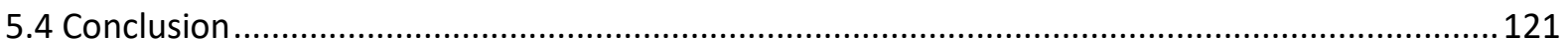

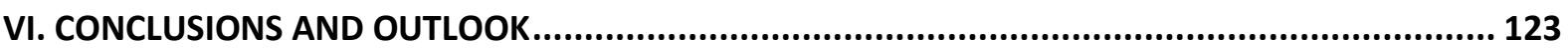

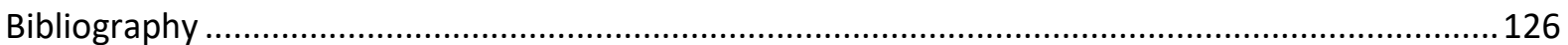

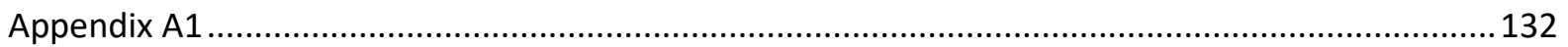

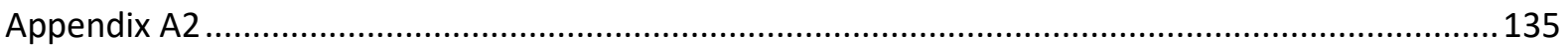




\section{Abstract}

The processes by which optical and electrical energies are transduced are at the heart of many modern technologies such as solar cells, light emitting diodes, photodetectors, imaging systems and displays. The basic functional element of these 'opto-electrical' devices are semiconductors, and the underpinning physics of how they transduce light and electricity is well understood for conventional inorganic materials such as silicon and gallium arsenide. However, new semiconductors such as the organics and the organohalide perovskites present additional opto-electrical questions and challenges since they are molecular solids with varying degrees of disorder and crystallinity.

The work described in this thesis addresses these new questions and challenges, particularly in relation to how existing solid-state physics concepts must be adapted to reliably predict and model material-and-device-level structure-property relationships and performance. Two basic technology platforms are examined in detail - solar cells and light emitting diodes, with particular reference to so-called reciprocity. A second focus of the discussion is accurate determination of optical constants for these new semiconductors - a challenging endeavour due to factors such as morphological heterogeneity. Transfer matrix and drift diffusion formalisms are relied heavily upon to model, simulate and explain multi-layer device performance, and ellipsometry and spectrophotometry are utilised as the primary analysis and characterisation methodologies.

A new approach to optical constant determination is presented and validated, as is an adapted reciprocity framework for the linking of absorption, emission and charge transfer state characterisation in the presence of cavity interference. Several 'difficult' solar cell systems are analysed in detail - in particular the previously mysterious working principles of the so-called carbon-stack perovskite system are elucidated for the first time. These findings explain how an electrically non-selective contact can still function as an effective photovoltaic electrode dependent upon the local minority and majority carrier concentration profile.

The research described herein advances our understanding of next generation semiconductor opto-electrical physics and provides more practical means for the community to analyse optical constants. 


\section{Introduction}

This Chapter will discuss the motivation of the work described in this thesis, providing the context of the research as well as the general outline, introducing organic semiconductors, excitons, and the concept of second-generation and third-generation opto-electronic devices.

\subsection{Motivation and general overview}

The main focus of the work described in this thesis will be to the use of novel, organic and hybrid inorganic-organic (perovskite) semiconductors as the main active material in optoelectrical devices. The exploration of these materials is justified by certain salient features inherent to these systems such as elemental abundancy, suitability for flexible form factors, tuneable band gaps, and certain environmental and efficiency considerations 1234 .

The rise of atmospheric $\mathrm{CO}_{2}$ levels and the resultant effect on global warming ${ }^{5}$ has prompted scientists across the globe to look for more sustainable, alternative and renewable energy sources. One of the most promising candidates for additional energy sources is solar power, the conversion of solar radiation into useful electrical power. Much of the work discussed in this thesis will focus on next generation semiconductor use in solar cells, though it is also applicable to other opto-electrical semiconductor devices such as LEDs ${ }^{6}$, light emitting field effect transistors ${ }^{7}$, photodiodes and other optical sensors; indeed any device where the conversion of photons to charge carriers (or the reverse) is relevant.

The focus will be on certain organic and perovskite semiconductors (collectively next generation semiconductors) - quite different material systems but sharing some common features. Currently, the most widespread and used semiconductor materials are purely inorganic, most prominently silicon and gallium arsenide. Relatively abundant, tuneable by doping $^{8}$ and with efficient opto-electronics ${ }^{9}$, it is easy to see why these are dominant commercial materials. However, inorganic semiconductors do not come without their limitations, such as the weight and rigidity, the energy-intensive manufacturing process ${ }^{10}$, and the importance of the type of crystallinity when it comes to efficiency ${ }^{11}$. Many of these limitations in comparison to organics arise from the inorganic materials' crystalline nature.

Next generation semiconductor materials (which can be purely organic or hybrid materials which incorporate properties from both) tend to be more structurally disordered, though they 
sometimes exhibit low degrees of short-range crystallinity. This makes them excellent candidates for light or flexible devices ${ }^{12}{ }^{13}$, which have garnered increasing interest due to the increasing digitization and abundance of sensors and interactive elements in our everyday living space, a phenomenon which some are already calling the fourth industrial revolution ${ }^{14}$. Concrete examples include solar cells on clothing ${ }^{15}$, organic sensors ${ }^{16}$ and visible light transparent solar cells for use as windows ${ }^{17}$.

Another consequence of the disordered nature of these next generation semiconductor materials is a significant alteration in the electronic energetic structure on the molecular level. Rather than forming bands for charge carriers, which appear in crystalline materials due to the periodicity of the lattice (see for example the work of Kittel ${ }^{18}$ for a comprehensive explanation), disordered materials consist energetically of more separate, localized molecular orbitals. See Figure 1.1 for a visualization of the difference between the two situations banded semiconductors and so called molecular semiconductors.
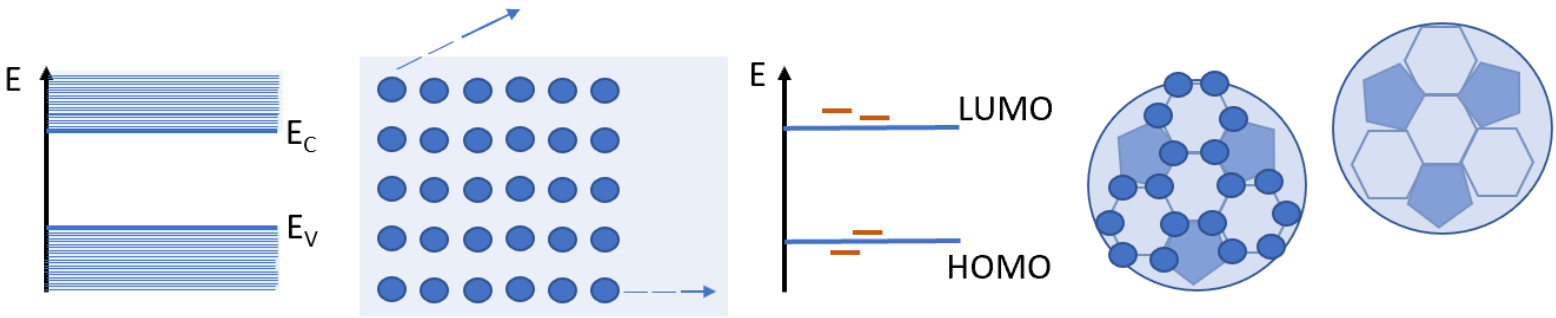

Figure 1.1 Band Structure and HOMO/LUMO

Figure 1.1. Left: Solid crystal with quasi infinite periodicity and electron wave function extending over the entire crystal. $E_{c}$ and $E_{v}$ are the energy levels delineating the conduction and valence band. Right: localized molecular orbitals such as in fullerene molecules $\left(\mathrm{C}_{60}\right)$. HOMO and LUMO are the highest occupied and lowest unoccupied molecular levels. The additional available levels for the HOMO and LUMO are vibrational states.

Charge transport in organic semiconductors in particular (more molecular in nature than the perovskites) therefore happens mainly through a mechanism called "hopping" ${ }^{19}$, where a charge carrier moves from orbital to orbital by energetic jumps. These jumps are usually well within the average energy available due to ambient temperature $(E=k T)$ at room temperature, which means they can be thermally assisted at a significantly high rate. Due to this high transfer rate from molecule to molecule, disordered semiconductors can still be 
practically treated as having a pseudo energetic band structure, by treating the highest occupied molecular orbital (HOMO) as if it were a conduction band, and the lowest unoccupied molecular orbital (LUMO) as a valence band ${ }^{20}$.

At this point it becomes important to discuss the charge carriers themselves. When a photon of sufficient energy impinges on a semiconductor material, an electron will be excited to a higher available energy level and take on the form of an "exciton", a quasi-particle that consists of an electron and a hole (the absence left by the electron). These two will be attracted to each other by the Coulomb force ${ }^{21}$, and behave as one neutral particle. To have net charge transport, it is therefore a requirement that the exciton is separated into its nonneutral components.

In the exciton dissociation process, the electron and hole must generally overcome their mutual Coulomb attraction, determined by the energy ${ }^{21}$ :

$$
E_{c}=\frac{q^{2}}{4 \pi \varepsilon_{r} \varepsilon_{o} r}
$$

Where $q$ is the elementary charge, $\varepsilon_{r}$ the dielectric constant (also called relative permittivity, this will be discussed more in-depth in chapter 3 ), and $r$ the average space between the electron and hole.

Excitons can be classified as two types, which can be considered edge cases depending on the dielectric constant of the semiconductor. If the dielectric constant is low, the electron and hole will be strongly attracted to each other and hard to separate. This is the so-called Frenkel exciton, which occurs for example in fullerenes, with a typical $\varepsilon_{r}$ of $2-3 \mathrm{~F} / \mathrm{m}$. However, if the dielectric constant is high, as in crystalline silicon (around $11.7 \mathrm{~F} / \mathrm{m}$ ), the electric screening of the material will make the coulomb interaction between the hole and electron weak, and the majority of excitons will spontaneously dissociate at room temperature. This type of exciton is called a Wannier-Mott exciton and is more delocalized.

Since organic semiconductors tend to possess a low-degree crystallinity and are generally made up of lighter elements compared to crystalline semiconductors, their dielectric constants $\varepsilon_{r}$ are lower. Because of this, transport in organic semiconductors is often excitonic (that is, charge will be transferred in the neat organic material as an exciton after generation). However, Wannier-Mott excitons (with spontaneous dissociation) can still exist in certain 
hybrid materials, provided the dielectric constant is high enough. This is for example the case with the hybrid organic-inorganic material the organohalide perovskites, which can have nonexcitonic charge photogeneration and transport ${ }^{22}$.

Therefore, from here on, an important distinction will be made: conventional organic semiconductor devices, with excitonic opto-electrical physics, will often rely on a blend containing fullerene to promote photogenerated charge dissociation. These will be called second-generation opto-electronic materials and related devices ${ }^{23}$. Any organic or hybrid semiconductor materials or devices deviating from this excitonic picture or with optoelectrical physics that we do not quite as yet understand (such as perovskites, non-fullerenes, or quantum dot devices) will be classified as third-generation ${ }^{23}$.

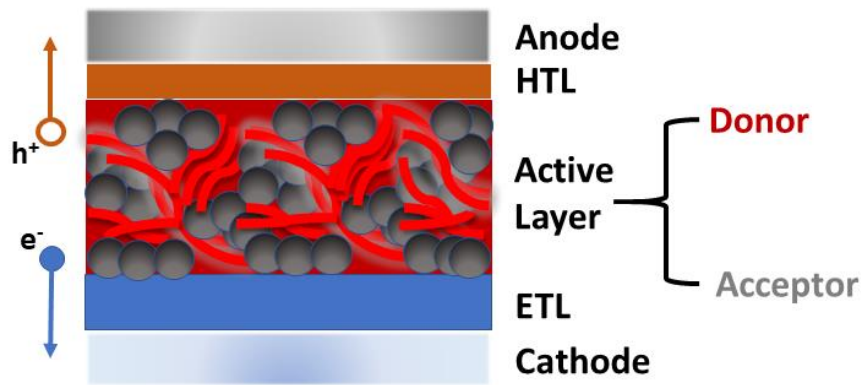

Figure 1.2 Blended Organic Structure

Figure 1.2. An example of an organic opto-electronic device with a bulk-heterojunction, containing a blend of two organic materials in the active layer, a fullerene acceptor and polymer donor. The hole transport layer $(\mathrm{HTL})$ is selective to holes, and the electron transport layer (ETL) to electrons.

Because exciton dissociation is a requirement for net charge transport, second-generation devices usually have two (or more) different organic semiconductors blended together at the nanoscale in the active layer - a pseudo p-type material called the donor and equivalent $n$ type called the acceptor (see Figure 1.2). This two component system is called a blend while the individual components are referred to as neat. The specific case of a donor-acceptor blend of organic semiconductors in a photovoltaic diode is called a Bulk-Heterojunction or BHJ. In a $\mathrm{BHJ}$, the energetic transport levels are lower for the electron acceptor material, or higher for the hole acceptor (or electron donor) material, in order to separate the excitons spatially (Figure 1.2). The contact area of two different organic materials leads to a unique energetic 
state known as the Charge Transfer state (CT state), which is an energetically stable state where the carriers of the exciton are partly occupying the donor molecular orbital and partly occupying the acceptor molecular orbital. It is assumed that the entropically larger amount of states available to the hole and electron will then lead to them diffusing apart spatially ${ }^{24}$. An energetic depiction of a CT state in a classic second-generation organic blend, PCDTBT:PCBM, is given in Figure 1.3.

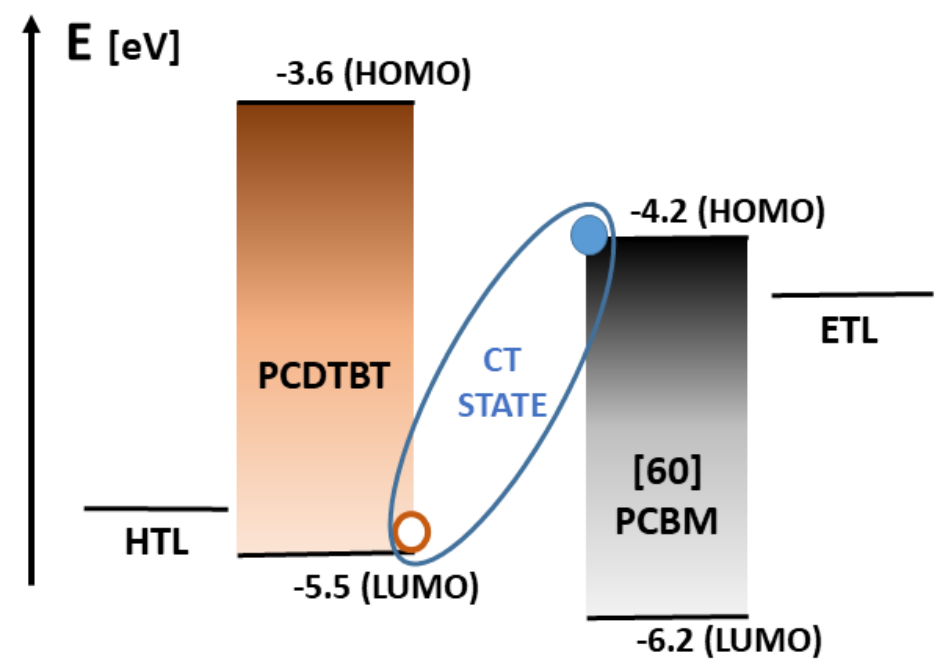

Figure 1.3 Charge Transfer State

Figure 1.3. Depiction of $C T$ state in a solar cell with $\mathrm{PC}_{60} \mathrm{BM}$ :PCDTBT active layer material. HOMO/LUMO levels of PCDTBT (donor material) and PCBM (acceptor material) are defined levels at the top and bottom. CT state is shown in blue.

While second-generation opto-electrical devices that rely on fullerene materials are still a growing field under much investigation, the true growth over the last few years has taken place in so-called third generation materials and devices ${ }^{23}$, where the highest recent breakthroughs in organic and hybrid device efficiency have taken place. These devices come with new challenges when trying to model and improve them, because of the exceptional properties of the novel semiconductor materials involved. The two materials that will be examined in detail in this work are perovskites and non-fullerenes.

Perovskites are a broad class of materials with a crystal structure identical to that of calcium titanium oxide $\left(\mathrm{CaTiO}_{3}\right)$. The exact perovskite crystal structure is depicted in Figure 1.4. When talking about perovskite in opto-electrical devices, we often refer to perovskites which 
incorporate some organic component in the structure, usually Formamidinium (FA) or Methyl Ammonium (MA). In other words, it is a hybrid organic-inorganic semiconductor material. Perovskites have a particular stoichiometry where a unit cell can be chemically denoted as $A B X_{3}$. In the case of the organic hybrid perovskites, the organic component will be the central molecule of the unit cell, $A$. The result is a semi-crystalline material, with a somewhat mobile organic molecule in the centre of a slightly more stable inorganic crystal.

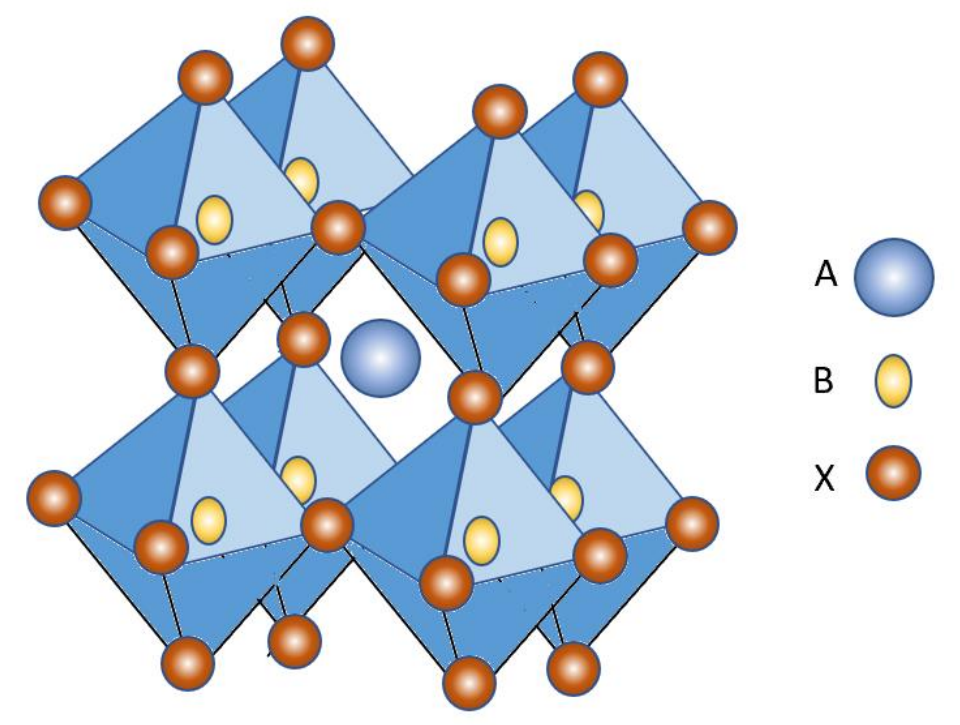

Figure 1.4 Perovskite Crystal

Figure 1.4. Typical depiction of the perovskite crystalline structure. The most widely recognized example for third-generation devices is $\mathrm{MAPbl}_{3}$, where $A$ is $M A, B=P b, X=I$.

Depending on what ions or molecules are used, the crystal can become more or less energetically stable and absorb different wavelengths of light (i.e. have different bandgaps) 25. Stability is a key property for perovskites, and indeed while the energetic conversion efficiency (from incident photons to collected carriers) has been improving significantly over the recent years, claiming much higher numbers than conventional organics of the second generation, it is this key property of stability ${ }^{26}$ that still requires significant investigation. Most perovskites degrade when in contact with water and oxygen, and various attempts have been made to account for this $25,27-29$.

It is the semi-crystalline nature of perovskites that simultaneously may account for the high efficiencies ${ }^{30}$, and for the stability issues. Recent studies have shown the additional 
complication of ionic mobility within the perovskite structure ${ }^{31}$, where interstitial ions can move through the lattice when an external electric field is applied. This may explain why many perovskite devices exhibit light soaking effects ${ }^{32}$, where the response to an applied illumination (or voltage) changes gradually over time.

Aside from these unusual ionic movement and stability complications, perovskites are also not excitonic, unlike most of their second-generation counterparts. Due to the high dielectric constant of perovskites, the exciton dissociation energy is low enough for near spontaneous dissociation at or near room temperature ${ }^{33}$. The carrier mobility of perovskites is also much higher than in organic semiconductors, again most likely due to this semi-crystalline structure. This allows the fabrication of optically thicker devices and also means that unlike for conventional organics, most of the recombination occurs at the interface with other layers, where lattice mismatch can create trap states, rather than within the bulk ${ }^{34}$. There exist several variations where different kinds of perovskites are mixed, or where the crystalline structure is manipulated, or where mesoporous blends are again created. This will be discussed in Chapter 4.

The so-called Non-Fullerene electron Acceptors or NFAs are a family of n-type organic semiconductors that have emerged relatively recently and delivered a step change in organic solar cell performance. They differ from second-generation organics in the fact that they do not use fullerene based acceptor materials. Some of the common materials that have recently come under investigation are ITIC, Y6 and related families that work in combination with blend partners such as PM6, PCE10 and PCE12 ${ }^{35}$. A key difference for non-fullerenes is that the acceptor can also absorb on the low-energy part of the spectrum and can thus be coupled with different kinds of donor materials, which absorb mostly in the high-energy regime instead. The NFA element of the blend in organic solar cells is often the narrowest gap component and thus defines the effective optical gap of the system.

Perhaps even more importantly, for some NFA blends the energy off-set between levels in the CT state (either $\triangle \mathrm{HOMO}$ or $\triangle \mathrm{LUMO}$ ) can be quite small, on the order of less than $0.3 \mathrm{meV}$ (see Figure 1.5). Initially this energy offset was thought to be vital for CT state charge separation, yet these materials still have a high charge separation efficiencies ${ }^{36}$. While in fullerenes, excitons excited in the fullerene usually have a low CT state charge separation efficiency and thus contribute little to the collected charge, NFA blends can have significant 
contributions from both materials. Moreover, non-fullerenes may also exclude triplet exciton loss pathways in certain materials ${ }^{37}$.
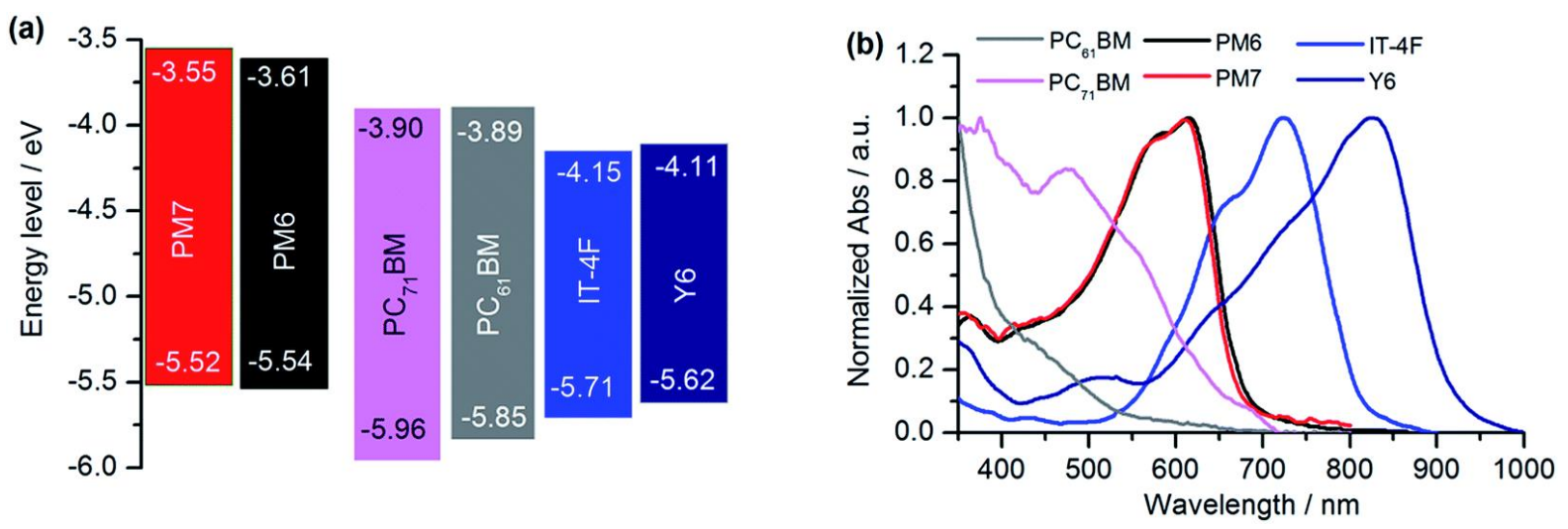

Figure 1.5 Non-Fullerene HOMO/LUMO

Figure 1.5. a) Example modified and taken from ${ }^{38}$, showing the unusual HOMO/LUMO energy off-sets of non-fullerene materials (PM6 and PM7 are donors, IT-4F and Y6 acceptors), compared to fullerene (PCBM) HOMO/LUMO levels. b) absorption spectra of the materials.

Another important property which applies to both non-fullerenes and perovskites, is that the optical constants of these materials tend to be significantly higher than with secondgeneration organic materials. This is due to the fact that fullerenes have the buckyball molecular structure and thus stack at lower density than the planar non-fullerenes and perovskites. A significant consequence of this is that the dielectric constants within these materials are also usually much higher, making the exciton dissociation energy in NFAs is much lower (see Eq. 1.1), though not so low as to facilitate spontaneous separation at room temperature in the neat material as with perovskites ${ }^{33}$. In that sense NFAs are still excitonic materials but we still classify them as third generation since they seem to have some new intermediate properties.

The typically high optical constants also make the use of conventional experimental methods such as ellipsometry problematic. The fitting solution space in ellipsometry becomes smaller with higher optical constants, making a good fit more challenging to find. A first key challenge in characterizing these third-generation materials is therefore that additional methods need to be found to determine the optical constants. Two additional methods were developed during the work described in this thesis precisely for this purpose: a spectroscopic transmittance method, which will be discussed in the Chapter 3, and dynamic infiltration 
ellipsometry, which will be discussed in Chapter 4. This work is an example of the clear motivation of the research, and an attempt to shed more light on these exciting new semiconductors.

\subsection{Conclusion}

This chapter introduced the concept of second-generation and third-generation semiconductor materials, and their use in photovoltaic devices. These materials are being investigated because of their elemental abundancy, flexibility, light weight and tuneable absorption properties. The general concept of photovoltaic charge generation as a key subset of opto-electrical devices that utilize semiconductor materials was introduced: firstly, the concept of excitons and a basic description of the types of excitons, the Frenkel and WannierMott types. A basic overview of the energetic structure of crystalline and amorphous semiconductors was also outlined, showing how next generation more disordered semiconductors have localized, molecular orbitals rather than the delocalized band structures of crystalline semiconductors. This different energy structure was then shown to require the use of blends for improved exciton dissociation, at least in the case of Frenkel excitons and resulting excitonic transport in the neat material. This gives rise to a new type of state for the charge carriers called the charge transfer state. Finally, the general class of organic semiconductors utilizing fullerenes was extrapolated to a class of so-called third-generation materials, including the hybrid class of perovskite materials, and non-fullerenes. These materials specifically will be the focus of the work described in this thesis. We will slowly work our way up to the opto-electronics of these materials and related devices and their challenges, starting from the reciprocity principle for regular, inorganic semiconductors, to organic blend devices, and finally to the perovskites and non-fullerenes. 


\section{Theory of opto-electrical devices}

This chapter will present a theoretical framework for characterizing opto-electrical interactions in devices which utilize new, emerging types of semiconducting materials. It is therefore useful to first consider the theory for conventional, inorganic opto-electrical materials and devices. The main part of this will be based on the reciprocity principle. Afterwards we will discuss how this theory diverges for second-generation organic semiconductors. Additional theory will be supplemented to deal with these challenges, most notably the transfer matrix method.

\subsection{Inorganic semiconductor devices}

\subsubsection{Shockley-Queisser limit}

In any opto-electrical device, the fundamental process that must be characterized is the conversion between photonic and electrical energy or vice versa as defined in Chapter 1 . As with all conversions of energy, the equilibrium of this process can be determined through thermodynamics. In particular, a thermodynamic principle called "Detailed Balance" can be applied to gain a fundamental basis for the electro-optical dynamics within a semiconductor material and related devices. The Detailed Balance principle states that if a system is in thermal equilibrium with its environment, then all microscopic processes within the system are exactly counterbalanced by their inverse microscopic process, a phenomenon that follows directly from the Second Law of Thermodynamics ${ }^{39}$. There are alternative ways of stating the principle of Detailed Balance, but for the scope of our considerations this will be the most practical one.

As described in Chapter 1, all opto-electrical devices, such as light emitting transistors, photodetectors, light-emitting diodes and solar cells, make use of semiconductor materials as the functional component(s). These materials have a conductivity that can be tuned via various parameters, such as temperature, ion doping, impurities, and crystallinity. In conventional, inorganic semiconductor materials (such as Silicon or Gallium Arsenide), the key characteristic that determines the optical properties of the material is the band gap, the energetic difference between valence and conduction band. 
Let us consider the example of a semiconductor material within a solar cell (photovoltaic diode) and assume this diode to be in thermal equilibrium with its environment (see Figure 2.1a). Application of Detailed Balance implies that, in the dark, all blackbody radiation which is going into the cell and generating charge, will be equally counter-acted by recombination and emission of light out of the cell, within each photon energy interval $d h v$ (where $h$ is Planck's constant and $v$ the frequency). Blackbody radiation is here simply described by Planck's Law. In this simplified radiative limit, where we do not consider non-radiative recombination within the cell, it then becomes possible to quantify the generation rate in terms of the blackbody radiation:

$$
G=R=\frac{2 \pi}{h^{3} c^{2}} \int_{0}^{\infty} \frac{\alpha(h v)(h v)^{2}}{\exp \left(\frac{h v}{k T}\right)-1} d h v
$$

where $\mathrm{G}$ and $\mathrm{R}$ are the generation rate and recombination rate per unit area and per solid angle within the cell. A more detailed derivation of this is given in the work of Würfel ${ }^{40}$.

Commonly, we assume that the absorption probability term $\alpha(h v)$ equals 1 for photons above bandgap energy and zero for photons below it. We can then derive a formula for the dark current density $J_{O}$ in the cell within this radiative limit, by considering that this photon flow formula is valid at every energy $d h v$ (or $d E$ ), due to Detailed Balance:

$$
J_{0}^{r a d}=q \frac{2 \pi}{h^{3} c^{2}} \int_{E_{g}}^{\infty} \frac{E^{2}}{\exp \left(\frac{E}{k T}\right)-1} d E .
$$

Note that $E_{g}$ here denotes the bandgap energy. Eq. 2.2 can also be approximated as

$$
J_{0}^{r a d}=q \frac{2 \pi}{h^{3} c^{2}} \int_{E_{g}}^{\infty} E^{2} \exp \left(\frac{-E}{k T}\right) d E .
$$

The dark current density in this equation is generated by blackbody radiation and will be equal and opposite to the recombination current density, as per the principle of Detailed Balance. 

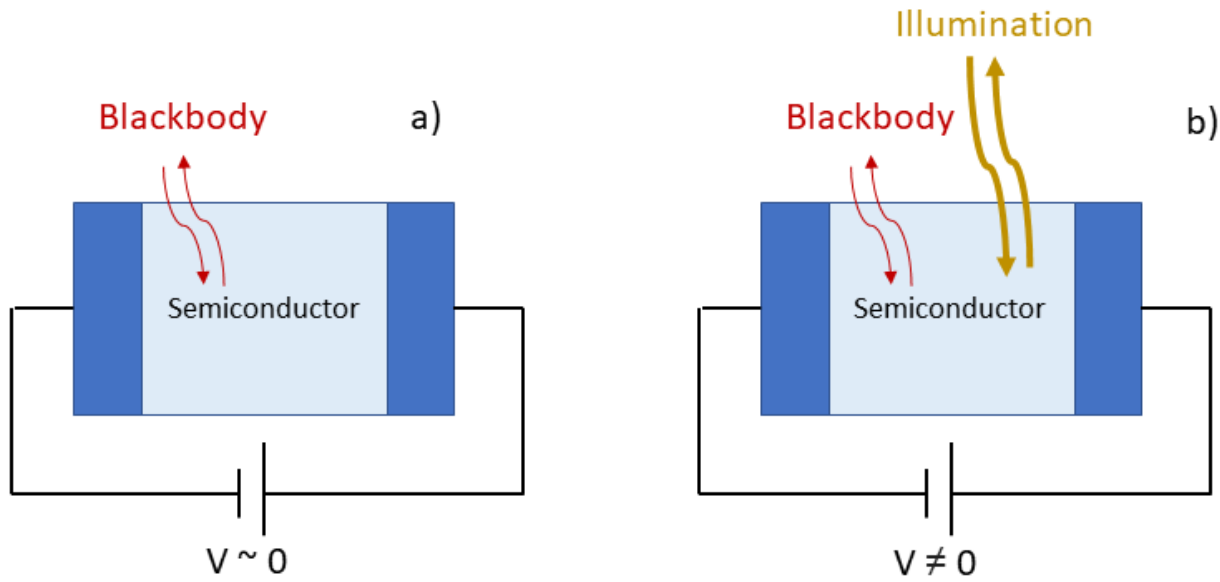

Figure 2.1 Blackbody Radiation Scheme

Figure 2.1 Diagram of a simplified solar cell device, a semiconductor sandwiched between two electrodes, a) under dark conditions when only blackbody radiation acts upon the cell and $b$ ) in illuminated conditions.

Let us now consider a second case where the cell is illuminated by the sun (Figure 2.1b). This is the case discussed in the seminal work by Shockley and Queisser ${ }^{41}$, which presented a fundamental, radiative limit for energy conversion in solar cells. In this work, an efficiency limit is calculated for solar cells, only using the principle of Detailed Balance and certain, fundamental parameters such as the temperature of the sun, temperature of the environment, and most importantly the bandgap of the semiconductor. Under solar illumination, extra charge will be generated in the active layer of the solar cell and build up a solar cell voltage $V$, which can be measured over the contacts of the cell. This voltage can be seen as a pseudo chemical potential, which is added to the energetic band potential felt by the free charge carriers. Shockley and Queisser even note in their work that every form of energy acting upon the carriers in the cell can be expressed in terms of such a voltage ${ }^{41}$ :

$$
\begin{gathered}
E_{g}=q V_{g}=h v_{g} \\
k T_{c}=q V_{c}
\end{gathered}
$$

Where the variables $E_{g}$ and $T_{c}$ denote the bandgap energy and the cell temperature, respectively. When considering a modified energy which includes the chemical potential from the cell voltage $V$, the Boltzmann factor in Planck's Law will be modified to arrive at a new equation for the generation and recombination rates. The voltage-modified version of Eq. 2.1 then becomes: 


$$
G=R=\frac{2 \pi}{h^{3} c^{2}} \int_{0}^{\infty} \frac{\alpha(E)(E)^{2}}{\exp \left(\frac{E-q V}{k T}\right)-1} d E
$$

This modification can also be applied in terms of Fermi energy levels, which are virtual, modified band levels as a function of carrier concentration and (in the case of quasi Fermi levels) voltage. This is particularly useful when dealing with doped active materials, where the free carrier concentration is doped to be higher or lower than equilibrium, or when considering an internal electric field that varies with position in the active layer. A more indepth look at the effects and uses of modifying the Fermi level within the cell can be found in Würfel ${ }^{40}$, however for now it will suffice to simply consider Eq. 2.6.

Eq. 2.6 can be approximated again and given as the recombination current density, now under illumination:

$$
J^{r a d}=\frac{2 \pi q}{h^{3} c^{2}} \int_{E g}^{\infty} E^{2} \exp \left(\frac{q V-E}{k T}\right) d E .
$$

Note that we can isolate the dark current density in this expression, and the recombination current density then becomes

$$
J_{R}^{r a d}=J_{0}^{r a d} \exp \left(\frac{q V}{k T}\right)
$$

Under standard operation of a solar cell, some voltage is applied across the cell, but some current is also allowed to run through the contacts. Under short circuit conditions, where no voltage is applied across the cell, all the current generated by (non-blackbody) radiation will be siphoned off by the contacts. This is commonly called the short circuit current $\left(J_{s c}\right)$. The full current density of the cell under operation will therefore consist of this short-circuit current and the current generated by the blackbody radiation, counter-acted by the voltagedependent recombination current of Eq. 2.8.

The full expression for current density then becomes:

$$
J^{r a d}=J_{0}^{r a d}\left(\exp \left(\frac{q V}{k T}\right)-1\right)-J_{s c}
$$


This is the commonly used $J V$ equation in the radiative limit. Similarly, this equation can be reworked to give the open-circuit voltage, where the generation and recombination current densities are equal, and the cell is therefore equivalent to an open circuit where no current flows:

$$
V_{\mathrm{oc}}=\frac{k T}{q} \ln \left(\frac{J_{\mathrm{sc}}}{J_{0}^{r a d}}+1\right)
$$

A typical, measured $J V$ curve of a solar cell under artificial 1 sun illumination (Air Mass 1.5 Global, or AM1.5G) is given in Figure 2.2a. A theoretical JV curve, given by Eq. 2.9, can be seen in Figure 2.2b. Some notable differences between the two are that the real $V_{\text {oc }}$ is lower than predicted (this is where the $0 \mathrm{~A} / \mathrm{cm}^{2}$ line is intersected), and that the current starts declining sooner than expected (this loss is typically measured in terms of fill factor, the ratio of measured power $P_{\mathrm{MP}}$ over $\left.J_{\mathrm{sc}} V_{\mathrm{oc}}\right)$. Both losses can be explained by the fact that we have not considered non-radiative forms of recombination. The exact impact of this will be discussed later on.
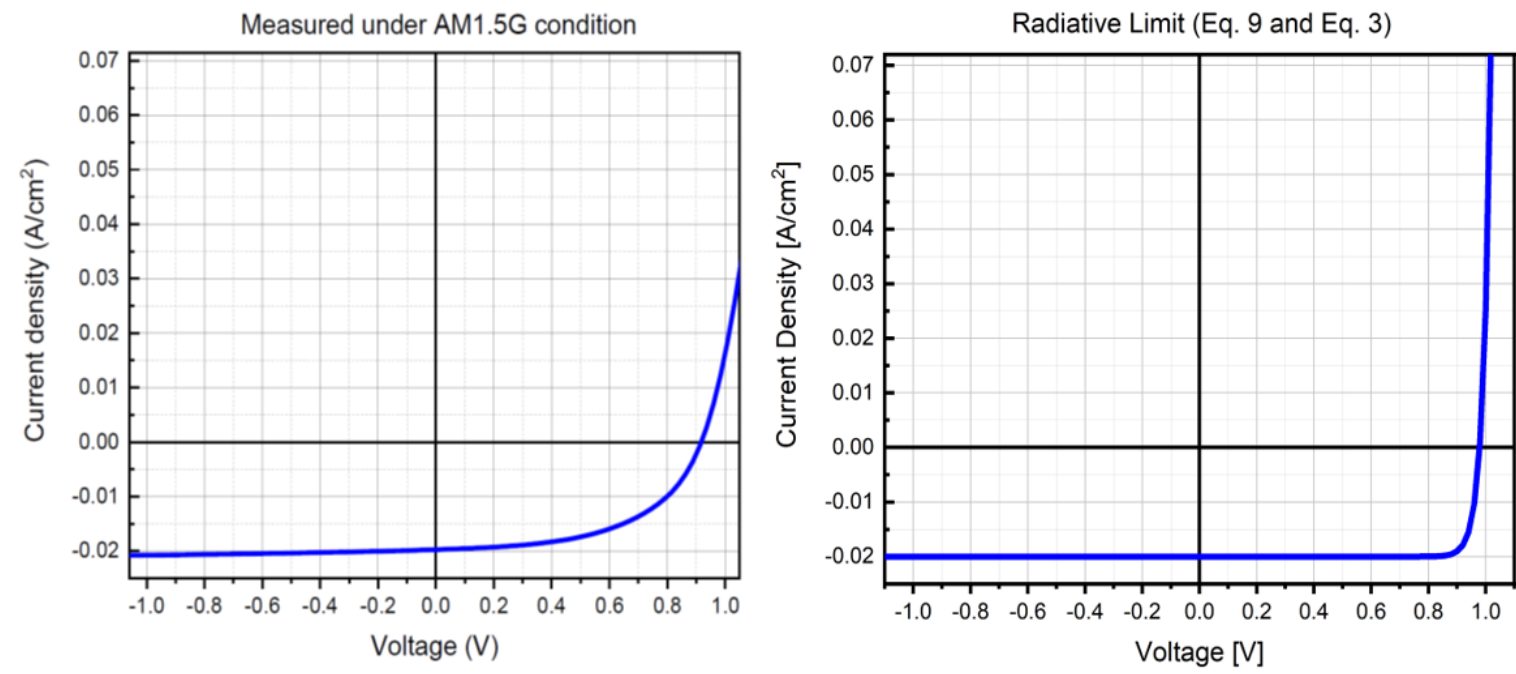

Figure 2.2 JV Curve Theory

Figure 2.2. Left: A JV curve, measured for a solar cell with a $1.25 \mathrm{eV}$ bandgap under AM1.5G. Right: Predicted $J V$ curve in the radiative limit for the same solar cell. Differences are due to non-radiative phenomena.

Using their radiative limit, Shockley and Queisser also showed that an upper limit for the quantum yield, the efficiency of extracted electrical energy over incident optical energy, can be calculated for any solar cell, depending on the active material's band gap. This limit was 
referred to as the Detailed Balance limit, although it is now commonly known as the ShockleyQueisser limit. The original figure is given in Figure 2.3, predicting a limit to the quantum yield $\eta$ as a function of the active material's band gap energy. For reference, today's best efficiency for a Silicon solar cell is $26.7 \%{ }^{42}$, not too far from what they originally predicted.

However, the theoretical limit considered in the work of Shockley and Queisser is still flawed for two main reasons. One is that they did not account fully for all the light in-coupling considerations, notably the parasitic absorptance of other layers. The other was the existence of non-radiative recombination phenomena. We will now look at both these problems in more detailed, modern terms.

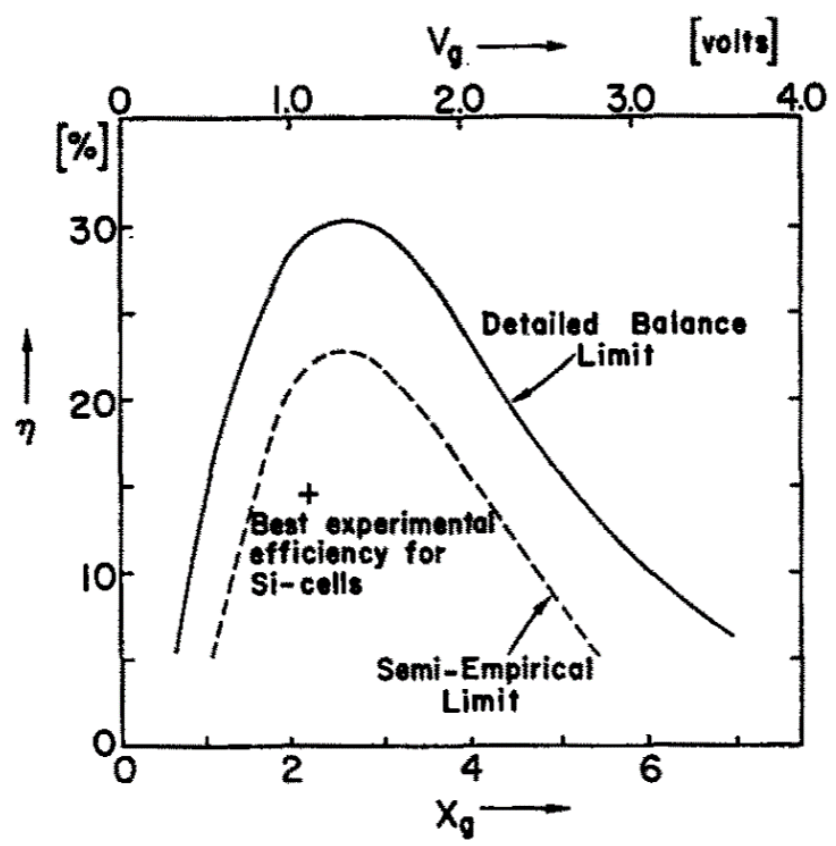

Figure 2.3 Shockley-Queisser

Figure 2.3. Modified and taken from ${ }^{41}$ : the original depiction of the Shockley-Queisser efficiency limit $(\eta)$ vs the efficiency records at the time, in terms of bandgap energy $\left(x_{g}, V_{g}\right.$, where $\mathrm{x}_{\mathrm{g}}=E_{g} / k T$ and $\left.V_{g}=E_{g} / q\right)$. Compare to today's top Si cells of $26.7 \%$.

\subsubsection{Light-coupling considerations}

The "quantum yield" mentioned previously is today referred to in two, more contemporary terms, namely External and Internal Quantum Efficiency, and these are some of the key metrics when looking at novel types of opto-electrical devices. The distinction between External and Internal Quantum Efficiency stems from the fact that, within the scientific 
community, a need has arisen to separate charge generation and the internal charge collection within a device.

Supposing a number of incident photons on the cell, $N_{i p}$, a number of photons absorbed in the active material, $N_{a m p}$, and a number of collected charge carriers $N_{c c}$, the External Quantum Efficiency (EQE) can be defined as the ratio between collected charge carriers and the incident photons on the cell, and the Internal Quantum Efficiency (IQE) as the ratio of collected charge carriers to absorbed photons in the active semiconductor material.

$$
\begin{gathered}
\mathrm{EQE}=\frac{N_{\mathrm{cc}}}{N_{\mathrm{ip}}} \\
\mathrm{IQE}=\frac{N_{\mathrm{cc}}}{N_{\mathrm{amp}}}
\end{gathered}
$$

Note that this implies that the IQE is included within the EQE and can be re-obtained from it:

$$
\mathrm{IQE} \cdot \frac{N_{\mathrm{amp}}}{N_{\mathrm{ip}}}=\mathrm{EQE} .
$$

This factor $\frac{N_{\mathrm{amp}}}{N_{\mathrm{ip}}}$ is effectively the fraction of incident photons absorbed in the active material layer, which we can denote as $A_{\mathrm{am}}$. The main effects causing a loss of incident photons are reflectance off the solar cell $R$, transmittance through the solar cell $T$, and parasitic absorptance by the other materials in the cell, here termed $A_{p}$. These are all fractional properties, they represent a fraction of the total number of incident photons as following:

$$
R=\frac{N_{\mathrm{rp}}}{N_{\mathrm{ip}}}, T=\frac{N_{\mathrm{tp}}}{N_{\mathrm{ip}}}, A_{\mathrm{p}}=\frac{N_{\mathrm{pmp}}}{N_{\mathrm{ip}}},
$$

Where $N_{\text {rp }}$ is the number of reflected photons, $N_{\text {tp }}$ the number of transmitted photons, and $N_{\text {pmp }}$ the number of photons absorbed by other layers (parasitic material absorptance). These fractions are also commonly given in intensity rather than number of photons. It follows that, supposing that all the above-mentioned pathways to photon flow are the only ones, the absorptance in the active material is given by:

$$
A_{\mathrm{am}}(\lambda)=1-R(\lambda)-T(\lambda)-A_{\mathrm{p}}(\lambda)
$$

And we can write the relation between IQE and EQE in Eq. 2.13 as follows ${ }^{43}$ : 


$$
\operatorname{EQE}(\lambda)=\frac{1}{1-R(\lambda)-T(\lambda)-A_{p}(\lambda)} \cdot \operatorname{IQE}(\lambda)
$$

This equation allows us to separate the electrical and optical properties of a solar cell, or indeed of any opto-electrical device. Thus, the quantum efficiency EQE of an opto-electrical device can be separated into an optical and an electrical component, the electrical being denoted by IQE.

The quantum efficiency is usually measured spectrally, and the EQE can be measured directly by setting up a lamp with a known Intensity (commonly one that emulates the spectral shape of solar radiation, such as AM1.5G), and measuring the collected charges from the cell as a functional of incident light wavelength (energy).

However, the IQE has to be deduced more subtly, for example by measuring more components of Eq. 2.15. IQE is a useful property because it characterizes charge collection efficiency inside the opto-electrical device and gives an indication of the electrical charge transfer processes in our materials. The EQE as a property is more ambiguous since it includes both (the charge transfer and the optical generation efficiency). In order to improve the device's efficiency, these electrical and optical processes inside the device must be considered separately, so bottlenecks can be found in order to attain higher efficiencies. This is especially true for new kinds of opto-electronic devices, where certain aspects of the optical or electrical processes may still be unknown.

The optical properties in Eq. $14\left(R, T\right.$ and $\left.A_{p}\right)$ are therefore important in any device investigation, since they characterize not only the optical aspects of the used materials, but also help to separate the electrical properties.

Reflectance and transmittance can be measured with an accurate spectrophotometer, but the absorptance of different materials within the cell can require more nuance than only a measurement. Until recently, many within the scientific community have been satisfied with treating the parasitic absorptance from other layers in the device with the Beer-Lambert law of absorption (or, indeed, with disregarding the parasitic absorptance altogether). The BeerLambert law can be given comprehensively as follows:

$$
\frac{I_{\mathrm{abs}, i}}{I_{0, i}}=A_{i}=\exp \left(-\alpha_{i} t_{i}\right)=\exp \left(-\frac{4 \pi k_{i}}{\lambda} t_{i}\right)
$$


Here, $A_{i}$ denotes the absorptance of layer $i$ in a stack of materials and $I_{0, i}$ is the light intensity incident to that layer. $\alpha_{i}$ is the absorption coefficient and $t_{i}$ the thickness of the layer. In alternative writing, $k_{i}$ denotes the imaginary part of the material's refractive index, the part responsible for absorption, and $\lambda$ is the wavelength of radiation. Eq. 2.16 assumes that there is a plane wave of light traversing our multi-layer structure of absorbing materials. In accordance with Maxwell's Equations, a 1-dimensional plane wave in a material can be given as

$$
\vec{E}(x)=\vec{E}(0) \exp \left(i \cdot \frac{2 \pi \overline{n_{l}}}{\lambda} x\right)
$$

$\vec{E}$ denotes the electric field and $\bar{n}_{l}$ the full refractive index of the material. The Lambert-Beer law can be recovered by assuming a plane wave and by using the proportionality of intensity with $|E|^{2}$ (this will be derived more in-depth in Chapter 3 ). The problem with the plane wave assumption, however, is that in a multi-layer stack (or, in fact, in any single layer that does not stretch on infinitely) we are not always dealing with a plane wave. At every material boundary, there is a transmission and reflection of waves, and these reflections add new waves to the primary plane wave, which can create a completely altered field profile within the stack through the phenomenon of wave interference.

A good measure to consider if the plane wave approximation is valid is to check if the active material layer thickness is below twice the absorption length of the considered wave, where the absorption length $t_{a, i}$ is defined as the length over which the intensity of the beam will have dropped to $1 / e$, following Beer-Lambert:

$$
A_{i}=\frac{1}{e} \Leftrightarrow t_{a, i}=\frac{1}{\alpha_{i}}
$$

If the layer is more than twice the absorption length, at least $86 \%$ of the initial beam intensity will have been absorbed before it can be reflected at the next layer interface, and the absorption in the active material can be approximated with Beer-Lambert. This is the case for most inorganic semiconductor devices (such as $\mathrm{Si}$ and $\mathrm{GaAs}$ ), since they are characterized by high carrier mobilities and can therefore be extended to very thick layers without losing too much charge to recombination. In these devices, most of the light is absorbed before it can be reflected at the boundary. However, if there is a range of parasitic layers before the active 
layer, or if there are several, tuned active layers (such as in a tandem cell), even inorganic, conventional devices require a more nuanced model to acquire the correct absorptance in each layer. As we shall see later on, for next-generation devices with organic materials, which tend to be thinner, it will be absolutely vital to use formalisms which can describe standing wave profiles to calculate the generation and parasitic absorptance within the device. However, for now we will assume Beer-Lambert is valid for conventional devices.

This in effect allows us to solve Eq. 2.15 for the IQE if we have measured an experimental EQE. Once the IQE is known, we can make a prediction for the short-circuit current $J_{\text {sc }}$ from the carrier generation in the active layer. Finally, if the dark current is also measured (or calculated from a known material band gap, see Eq. 2.3), we can derive a radiative limit for $V_{\text {oc }}$ as well, using Eq. 2.10.

It is in fact possible to reconstruct the entire $J V$ curve, but for this, non-radiative losses must also be accounted for, in order to determine the Fill Factor, mentioned before as the ratio of maximum power of the cell to the maximum ideal power:

$$
F F=\frac{P_{\mathrm{MP}}}{V_{\mathrm{oc}} J_{\mathrm{SC}}}
$$

However, to quantify these losses, a formalism is required to quantify the charge transfer within the device after generation, and the electrical processes at play. 


\subsubsection{Charge collection}

a)

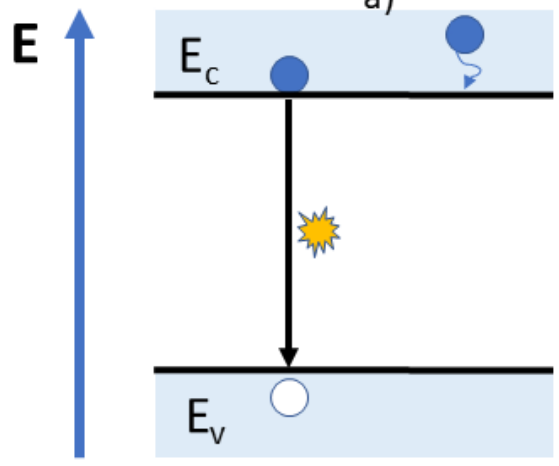

b)

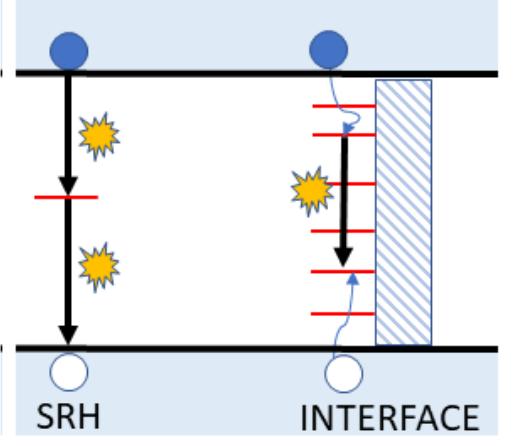

c)

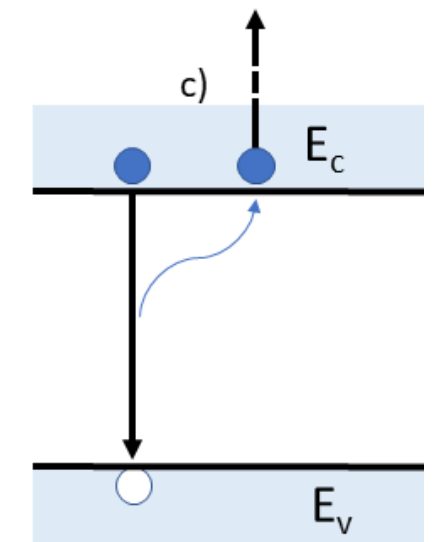

Figure 2.4 Recombination Overview

Figure 2.4. Schematic overview of processes which influence internal charge collection efficiency. From left to right: a) radiative recombination and non-radiative recombination, b) Shockley-Read-Hall (trap assisted) recombination and interface recombination, c) Auger recombination.

Until now we have mainly discussed the optical side of our devices: the radiative limit, the light-coupling phenomena, and the parasitic layer absorptance. Using these considerations, we arrived at a way to derive the IQE from Eq. 2.15, which characterizes the charge collection efficiency after charge generation. Now it is time to have a more in-depth look into these charge collection processes.

In a steady state, the charge transport within semiconductor materials can be characterized through the drift-diffusion current equations and the continuity equations, combined into the following form:

$$
\begin{aligned}
-\frac{\delta}{\delta \mathrm{x}} \cdot\left(\mu_{n} \cdot n(x) \vec{\varepsilon}(x)+D_{n} \frac{\delta n(x)}{\delta x}\right) & =G_{n}(x)-R_{n}(x), \\
\frac{\delta}{\delta \mathrm{x}} \cdot\left(\mu_{p} \cdot p(x) \vec{\varepsilon}(x)-D_{p} \frac{\delta p(x)}{\delta x}\right) & =G_{p}(x)-R_{p}(x),
\end{aligned}
$$

where $q$ is the elementary charge, $n$ and $p$ the respective carrier concentrations of electrons and holes, $\mu$ denotes the mobility and $\vec{\varepsilon}(x)$ is the internal electric field at position $x$. The second term is a diffusion term where $D$ is the diffusion coefficient, and $G$ and $R$ are the generation and recombination rates of free carriers. The actual transport of carriers has two 
main terms, a drift term that depends on the internal field, and a diffusion term that depends on the differential in concentration of free carriers.

From Eq. 2.20 it is also apparent that there is only one term preventing collection (and therefore impacting the IQE: $R(x)$, or recombination.

A schematic overview of all possible forms of recombination is given in Figure 2.4. The first is simple radiative recombination from the conduction band to the valence band, which reemits the absorbed light (the so-called band-to-band recombination). This also includes a nonradiative component of relaxation within the band, where energy levels are closely packed, and thermal relaxation is more viable. The second process makes use of available trap energy states within the band. Trap states may arise within the bulk material due to impurities, or at different material interfaces, due to lattice mismatch. Typically, at an interface there are more trap states. Both examples are shown, they can be radiative or non-radiative. Finally, there is Augur recombination, a non-radiative-type process where instead of radiating the recombination energy, energy is transferred to a free carrier which is propelled to a higher energy level.

Note that all the forms of radiative recombination can still be accounted for in the already considered radiative limit. We can take the previously derived Eq. 2.6 and insert a more nuanced absorption coefficient $\alpha(E)$, which also considers the additional available states within the bandgap (see Figure 5). However, the Detailed Balance principle used to derive Eq. 2.6 still only considers radiative absorption and emission between these levels to affect the charges. In reality there are also non-radiative energetic transfer processes, converting energy in the form of vibrational phenomena (i.e. thermal energy states or phonons). Most charges that are excited to available states above the bandgap will relax vibrationally to the lowest energy level of that band before being collected or recombining, since this nonradiative relaxation occurs much faster (this can be explained by the Franck-Condon principle $\left.{ }^{44}\right)$. This is not accounted for in the radiative Shockley-Queisser limit.

These vibrionic processes can however be incorporated as microscopic processes in Detailed Balance considerations, to derive a more complete theory, and an in-depth consideration of this can be found in the work of Kirchartz ${ }^{45}$. However, for this work, it will suffice to know 
some of these mechanisms exist and cause additional, non-radiative losses, which will also be contained within the IQE derived through Eq. 2.15.

The mobile charge carrier concentration in the semiconductor (that is, holes in the valence band and free electrons in the conduction band) will at all times have a certain average, probabilistic energy level, the Fermi level. For an intrinsic semiconductor with intrinsic conduction/valence band carrier concentration this is approximately in the middle of the two bands, however usually one or both of the bands are doped to form an extrinsic semiconductor, in which case, if we denote acceptor dopant concentration $\mathrm{N}_{\mathrm{v}}$ and donor dopant concentration $\mathrm{N}_{c}$, this Fermi energy level is given as:

$$
E_{f}=\frac{E_{c}+E_{v}}{2}+\frac{k_{b} T}{2} \ln \left(\frac{N_{v}}{N_{c}}\right)
$$

The Fermi level is often modified in conventional opto-electrical devices, usually through techniques such as doping, as outlined above, or with a voltage (or internal electric field), applied throughout the semiconductor layer (remember that voltage can be seen as a chemical potential, modifying the band energy levels). In these non-equilibrium conditions, we speak about separate quasi Fermi levels for both types of carrier. In conventional optoelectronic active materials such as Silicon, $\mathrm{p}$ - and n-type doped materials are brought in contact which creates a so-called space charge region, where an internal field (created by the depletion of majority carriers in this region) serves to sweep away light-generated carriers to the contacts.

Note that no matter what the band structure is, the mechanism decreasing charge collection will always be recombination. If charge is collected more slowly (due to low mobility or a counter-productive internal field) or if there are additional energy states within the gap, the probability for charges to recombine within the cell before collection increases ${ }^{46}$. In the case of non-equilibrium carrier concentrations, where one carrier concentration is significantly higher than the other (this is sometimes called low-injection), recombination rate can be given as a function of majority carrier off-set from equilibrium, $\Delta n$ :

$$
R=\frac{\Delta n}{\tau}
$$


Where $\tau$ is the carrier lifetime. This lifetime is often determined through emission experiments, which will be discussed more in-depth later on. Most variables in Eq. 2.20 often need to be determined experimentally, an essential undertaking when characterizing devices with new materials.

If the recombination is known, it can be linked to IQE. If the IQE were $100 \%$, all generated charge $\int G(x) d x$ would be collected as current at the contacts, however because there is a factor $\int R(x) d x$ that is not collected at the contacts, we have a non-perfect IQE:

$$
I Q E=\frac{\int G(x) d x-\int R(x) d x}{\int G(x) d x}
$$

Therefore, if we calculate the recombination rate profile from Eq. 2.20, the efficiency with which carriers are ultimately collected can also be derived, most notably the average carrier lifetime $\tau$ in the material.

We have now identified the main phenomena that can drive the efficiency of an electrooptical device. These are:

- The charge generation efficiency (CGE), related to optical effects such absorptance, reflectance and transmittance. Characterized by EQE, although it should be noted that IQE will also be included in EQE measurements and should be determined and isolated (see Eq. 2.15). Because it involves net charge, CGE is also influenced by exciton dissociation rate, but this is usually spontaneous for inorganics at room temperature.

- The charge collection efficiency (CCE), related to electrical recombination effects. Characterized by concepts such as the mobility, recombination rates (both in bulk and at interface). Can be modelled with drift-diffusion and continuity equations (see Eqs. 2.20). The IQE is mainly characterized by the CCE, but also by the exciton dissociation rate, which may become important later on.

Since the discussion until now has relied on thermodynamics and detailed balance, the same considerations are true for opposite opto-electrical devices in which certain types of radiative recombination are the favoured effect, in order to generate light, such as for Light-Emitting Diodes (LEDs). 


\subsection{Extension to organic semiconducting materials}

\subsubsection{Optical considerations: transfer matrix}

As discussed before, the main focus of the work described in this thesis will be to the use of novel, organic and hybrid organic-inorganic semiconducting materials as the main active material in opto-electrical devices.

As mentioned in Chapter 1, a key difference is that organic semiconducting materials are usually non-crystalline, or at least exhibit low degrees on long range order, and that charge transport therefore happens from molecular orbital to orbital, mainly through a mechanism called "hopping" ${ }^{19}$, where a charge carrier moves through discrete, energetic jumps. These jumps are usually well within $k T$ at room temperature, which means they can be thermally assisted at a significantly high rate. Due to this high transfer rate from molecule to molecule, organic materials can still be treated as having a sort of energetic band structure, by treating the highest occupied molecular orbital (HOMO) as if it were a conduction band, and the lowest unoccupied molecular orbital (LUMO) as a valence band ${ }^{20}$. However, because of this transport mechanism, organic semiconductors generally have a significantly lower mobility. Opto-electrical devices with organic materials will therefore always have much thinner layers, on a sub-micron scale, to prevent too much recombination during charge transport and collection.

Since active layers in organic opto-electrical devices are so thin, there will also be significant destructive or constructive interference within the active layer, caused by the reflectance off the multi-layer interfaces by light that is not fully absorbed due to layer width below the optical length (see Eq. 2.18). The interference will decrease or increase the absorptance of the device in the active layer and depending on the wavelength of incident light and thickness of the device, which creates standing waves. A formalism that can describe these standing wave profiles will need to applied.

The Abeles transfer matrix method is such a formalism, and allows one to compute the optical field distribution in any multilayer stack ${ }^{47}$. The only requirement for this method is accurate knowledge of layer thicknesses and complex refractive indices, which can be determined experimentally. The fundamentals of the methodology amount to the definition of a propagation matrix for each of the layers in an arbitrary stack as shown in Figure 2.5. 
To deal with the interference effects, we assume two independent waves propagating throughout the stack: a forward-propagating wave (here moving towards the right) and a backward-propagating wave. Note that these two independent waves already consist of the sum of all reflected and transmitted waves that are moving in the same direction. However, the sum of plane waves propagating in the same direction is still a plane wave:

$$
\begin{aligned}
& E_{1} \exp \left(i \cdot \frac{2 \pi \overline{n_{l}}}{\lambda}\left(x+\varphi_{1}\right)\right)+E_{2} \exp \left(i \cdot \frac{2 \pi \overline{n_{l}}}{\lambda}\left(x+\varphi_{2}\right)\right) \\
= & \exp \left(i \cdot \frac{2 \pi \overline{n_{l}}}{\lambda} x\right) \cdot\left[E_{1} \exp \left(\varphi_{1}\right)+E_{2} \exp \left(\varphi_{2}\right)\right],
\end{aligned}
$$

Which means the two considered waves (which are sums of all backward- and forwardspropagating waves) are both still plane waves. Using the Fresnel coefficients of reflection and transmission, $r_{i j}$ and $t_{i j}$ respectively, we can impose boundary conditions on these two waves. The Fresnel coefficients are given by

$$
\begin{gathered}
r_{i j}=\frac{\bar{n}_{i}-\bar{n}_{j}}{\bar{n}_{i}+\bar{n}_{j}}, \\
t_{i j}=\frac{2 \bar{n}_{i}}{\bar{n}_{i}+\bar{n}_{j}}
\end{gathered}
$$

where the indices ij denote the two consequent layers in the stack, and $\bar{n}_{j}$ is again the total refractive index of layer $j$, consisting of $\bar{n}=n+i k$ ( $k$ being the complex, absorbing part).

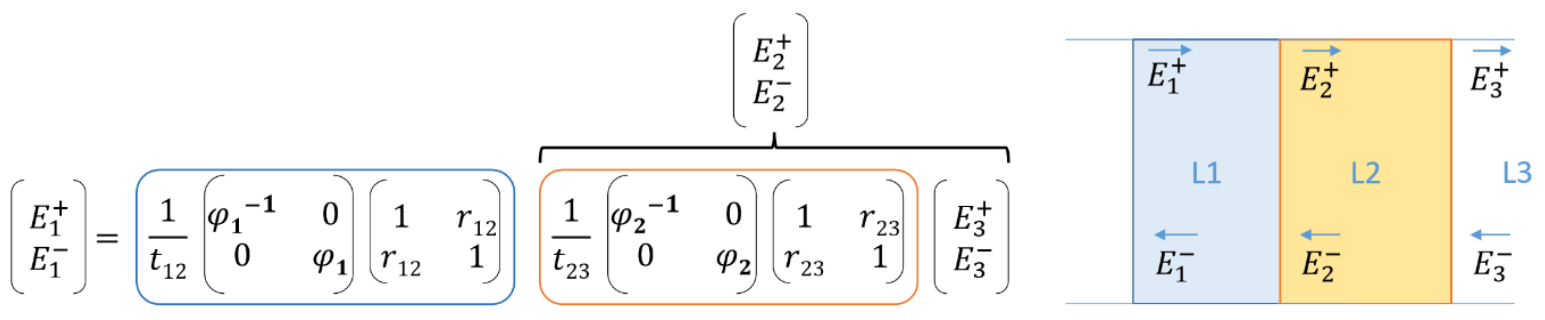

Figure 2.5 Transfer Matrix

Figure 2.5. Schematic overview of the transfer matrix method. Each layer has its own matrix, outlined in corresponding colour, and each matrix gives the relation between the Electric field magnitude of the forward- and backward-travelling wave at the start of the layer, and at the start of the next layer. 
Since the forwards and backwards propagating wave are both simple plane waves, this places a straightforward restriction on the Electric field magnitudes at the boundaries of adjacent materials: each plane wave at the start of a layer consists of

1. the transmitted wave of the previous layer and

2. the reflected wave of opposite direction from within the same material.

These relations can be re-obtained by working out the matrix products shown in Figure 2.5 . For layers 1 and 2 (outlined in blue in Figure 2.6):

$$
\begin{aligned}
& \vec{E}_{1}^{+}=\frac{1}{t_{12}}\left(\varphi_{1}^{-1} \vec{E}_{2}^{+}+\varphi_{1}^{-1} r_{12} \vec{E}_{2}^{-}\right) \\
& \vec{E}_{1}^{-}=\frac{1}{t_{12}}\left(\varphi_{1} r_{12} \vec{E}_{2}^{+}+\varphi_{1} \vec{E}_{2}^{-}\right)
\end{aligned}
$$

$E_{1}^{+}$is the field magnitude of the forwards wave at boundary 1 , and $E_{1}^{-}$that of the backwards wave. $\varphi_{1}$ is the phase propagation factor in layer 1 , equalling $\exp \left(i \cdot \frac{2 \pi \overline{n_{1}}}{\lambda} t_{1}\right)$, with $t_{1}$ the layer 1 thickness, and $\varphi_{1}^{-1}$ is the inverse phase factor $\exp \left(-i \cdot \frac{2 \pi \overline{n_{1}}}{\lambda} t_{1}\right)$. This can be re-written in more intuitive terms, noting that that $r_{12}=-r_{21}$ :

$$
\begin{gathered}
\vec{E}_{2}^{+}=\varphi_{1} t_{12} \vec{E}_{1}^{+}+r_{21} \vec{E}_{2}^{-} \\
\varphi_{1}^{-1} t_{12} \vec{E}_{1}^{-}=r_{12} \vec{E}_{2}^{+}+\vec{E}_{2}^{-}
\end{gathered}
$$

and Eq. $2.27 \mathrm{~b}$ can be rewritten using Eq. $2.26 \mathrm{a}$

$$
\begin{aligned}
& \varphi_{1}^{-1} t_{12} \vec{E}_{1}^{-}=\left(1-r_{21}^{2}\right) \vec{E}_{2}^{-}+r_{12} \varphi_{1} t_{12} \vec{E}_{1}^{+} \\
& \varphi_{1}^{-1} \vec{E}_{1}^{-}=\frac{\left(1-R_{21}\right)}{t_{12}} \vec{E}_{2}^{-}+r_{12} \varphi_{1} \vec{E}_{1}^{+} \\
& \varphi_{1}^{-1} \vec{E}_{1}^{-}=t_{21} \vec{E}_{2}^{-}+r_{12} \varphi_{1} \vec{E}_{1}^{+}
\end{aligned}
$$

Eq. 2.27a and 2.27c are simply the assumption of the continuity of plane waves throughout adjacent materials again.

Each layer effectively adds another matrix to the transfer matrix product, which lets us determine the forwards-and-backwards-propagating electric field $E_{i}$ at each layer interface. Thus, the optical field throughout the stack can be modelled if the layer optical constants are 
known. From this field distribution, we can then derive the charge photogeneration distribution in all material layers. The following equation ${ }^{48}$ can be used to calculate the absorptance per wavelength $\lambda$ at each position within each layer $i$ :

$$
I_{0, i} A_{i}(x, \lambda)=\frac{1}{2} c \varepsilon_{0} \alpha_{i}(\lambda) n_{i}(\lambda)\left|E_{i}(x, \lambda)^{2}\right|
$$

and by setting the incident to global AM1.5 radiation, we can then derive the generation of charge carriers:

$$
G_{i}=\int_{x_{i}}^{x_{i+1}} \int_{\lambda_{\min }}^{\lambda_{\max }} I_{A M 1.5}(\lambda) \cdot A_{i}(x, \lambda) d x d \lambda
$$

As we can see, the transfer matrix method lends itself to calculating both the parasitic and active material absorptances. We can also calculate the total reflectance and transmittance of the stack, by considering that $R=\vec{E}_{0}^{-} / \vec{E}_{0}^{+}$and $T=\vec{E}_{m+1}^{+} / \vec{E}_{0}^{+}$. Some extra considerations of the transfer matrix method, such as how to deal with incoherent layers in the stack (often the glass layer on top of the device is such a layer), can be found in Appendix A1.

Again, we can use the calculated parasitic absorbance, reflectance and transmittance in Eq. 2.15 to calculate the IQE of the cell from the EQE. This determination of a device's IQE becomes even more robust by measuring (rather than modelling) the total reflectance and transmittance of the cell, as could be done with an accurate spectrophotometer with specular and total reflectance capability and a suitably large-area device ${ }^{49}$.

It should be noted that the transfer matrix method is not the only method to describe cavity effects, and that while the transfer matrix presents a simplified and intuitive 2D understanding of a multi-layer stack, one could also calculate 3D modes within the device, using Finite Difference Time Domain (FDTD) software such as Lumerical or Comsol. This will play a role when investigating the emission of devices, where evanescent modes can become significant ${ }^{50}$. 


\subsubsection{Electrical considerations: blends}

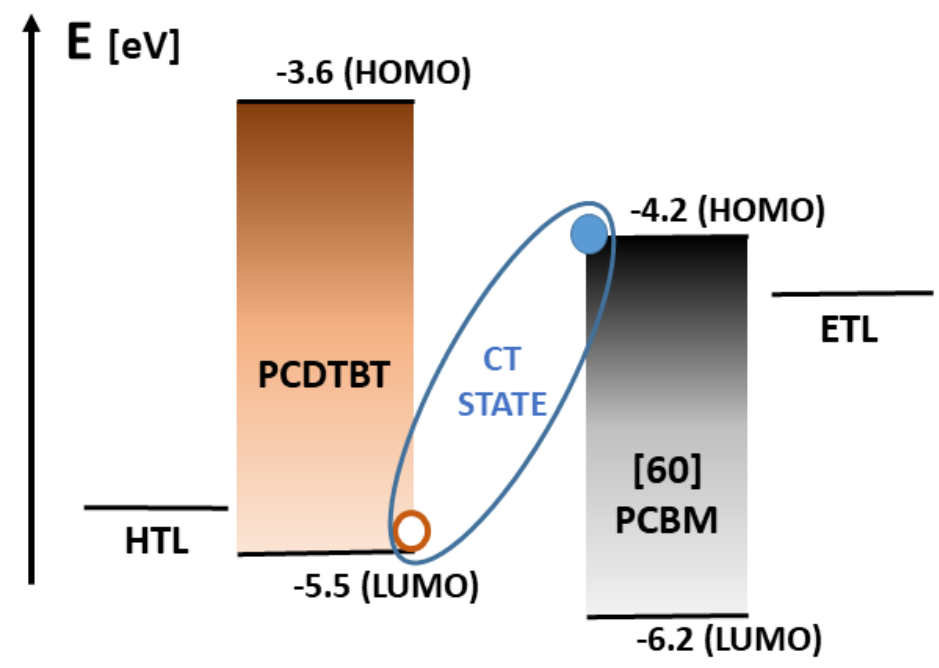

Figure 1.3. Depiction of $C T$ state in a solar cell with $\mathrm{PC}_{60} \mathrm{BM}: \mathrm{PCDTBT}$ active layer material. HOMO/LUMO levels of PCDTBT (donor material) and PCBM (acceptor material) are defined levels at the top and bottom. CT state is shown in blue.

In an organic blend layer such as depicted in Figure 1.3 (here repeated for clarity), the charge carriers now travel through two different active materials, with different recombination rates and mobilities. One would expect the device to be limited by the least efficient (that is, highest recombination rate) material, but there are two important aspects to the IQE (or collection efficiency) in the excitonic case:

1. The efficiency of exciton (and CT state) dissociation, which depends on many factors, such as the dielectric constant of the materials, but also the energy difference between the two HOMO or LUMO levels. For charge generated in the donor, a high difference in HOMO levels gives good exciton dissociation. For generated charge in the acceptor, it is the LUMO levels that matter. However, entropy also plays an important role, as will be seen later for the case of NFAs.

2. The recombination rate for holes in the donor and electrons in the acceptor materials of the blend, as well as at the material interfaces.

The most common acceptor material in second-generation cells is Phenyl- $\mathrm{C}_{\mathbf{x}}$-butyric acid methyl ester, or PCBM, a fullerene derivative where $x$ denotes the number of carbon atoms in the fullerene molecule. This material was chosen for its high electron mobility (around 3E- 
$3 \mathrm{~cm}^{2} / \mathrm{Vs}$ ), the ease of spin-coating it with solvents like Chlorobenzene (CB) and its stability with other materials, partly due to its high electron affinity and ability to stabilize more than 1 negative charge, and partly due to its spherical symmetry ${ }^{51}$. PCBM absorbs mainly at higher energies, at wavelengths above $600 \mathrm{~nm}$, and therefore needs to be combined with materials that start absorbing at lower energy, to maximize the absorbed part of the solar spectrum. The donor material in PCBM blends, be it PCDTBT, P3HT or any other p-type organic semiconductor, will therefore ideally be absorbing across the whole spectrum, but necessarily starts absorbing at the lower energy side of the spectrum. Absorption starts when the photon energy becomes greater than the difference between donor HOMO and LUMO levels. This would be the band gap in inorganic devices, however in the case of organics there are no actual bands with available state, only defined energy levels. At higher energy photons, higher energy excitons will still be generated (also sometimes referred to as 'hot excitons'), which will quickly relax through internal, thermal dissipation, usually in the form of phonons ${ }^{52}$. Most organics therefore still absorb significantly at higher energies than their HOMO/LUMO energy difference, just as an inorganic band structure would. See Figure 2.6 for some quantitative data for some of the most common second generation organic semiconductors HOMO and LUMO levels. 


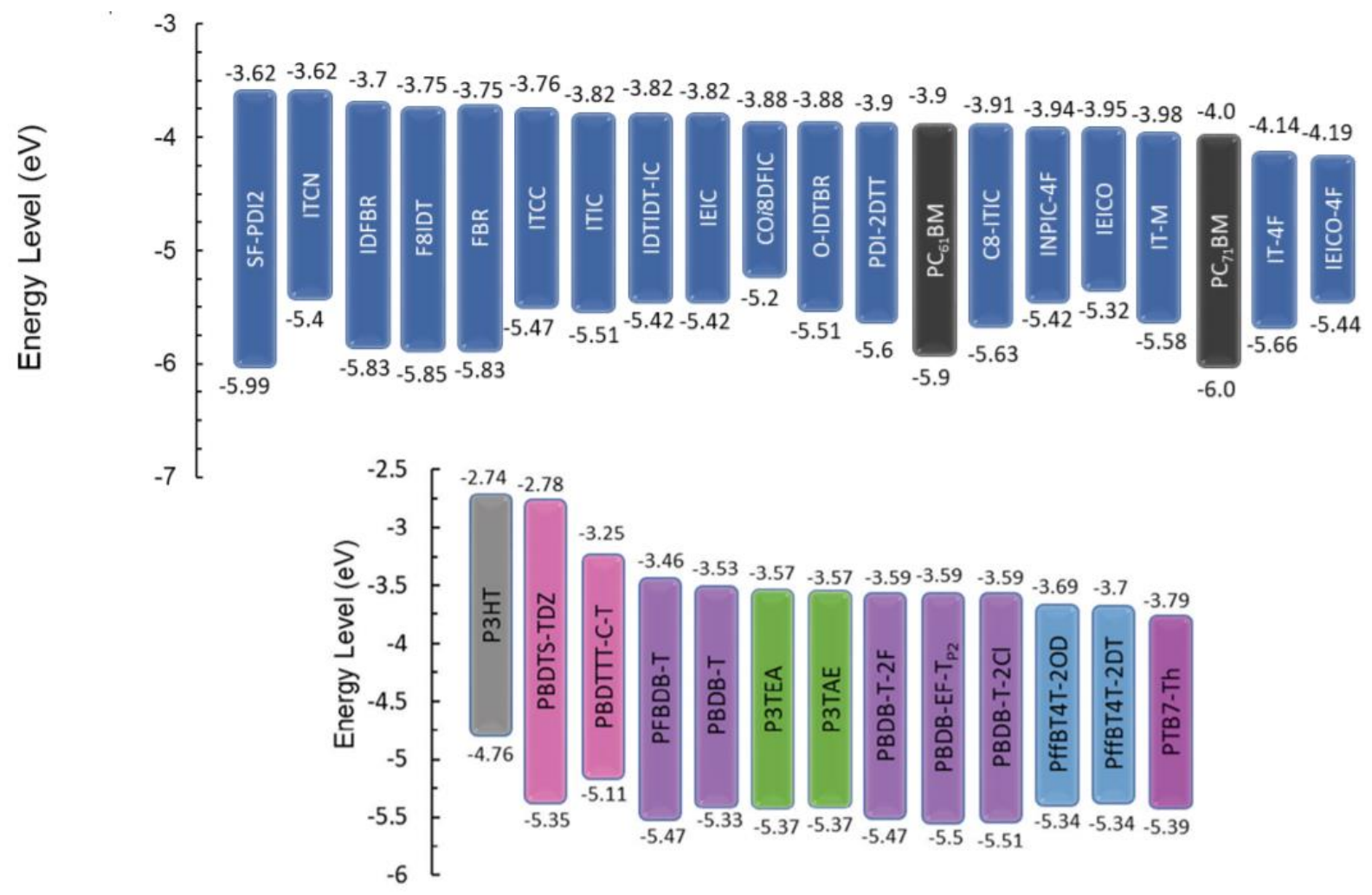

Figure 2.6 Overview of Common HOMO/LUMOs

Figure 2.6. Overview of the HOMO/LUMO levels of the most common organic semiconductors, demonstrating the diversity of materials available. Modified and taken from $^{53}$. Most of this data is available in the online Ossila database.

Although the two active materials will absorb light at different parts of the spectrum, as long as the exciton dissociation energy of the two materials is comparable, the IQE will be more or less the same across the spectrum, since the carriers from the dissociated excitons will always travel through their own respective material of the blend, no matter where they were first absorbed. For conventional organic devices, this condition of comparable dissociation energy tends to be true, and collection efficiency can be assumed to be quasi-constant across the spectrum (i.e. illumination energy independent), a useful assumption when trying to determine a device's IQE. There is still however a significant debate concerning so-called 'hot excitons' 54 which are photoexcitations produced by higher energies than the gap and may have a higher dissociation efficiency. However, there is little evidence of such hot effects under operational conditions, steady state and equilibrium ${ }^{54}$.

As mentioned before, organic materials can be treated as if the HOMO and LUMO are band semiconductors with a very low mobility, and because of this, the same current and continuity 
equations from Eq. 2.20 still hold. As a demonstration of pure excitonic transport, consider a neat organic material on glass (note that excitons are not dissociated here, as in a blend). The drift aspect of the equations is removed since excitons are neutral. We therefore have a neutral exciton flow density:

$$
-D_{e} \frac{\delta^{2} e(x)}{\delta x^{2}}=G_{e}(x)-R_{e}(x)
$$

Where $e(x)$ is the positional exciton concentration. This equation can be solved with appropriate boundary conditions to give some insight into the generation and recombination profiles in an organic semiconductor material (as compared to in a thick inorganic Silicon layer, where $G(x)$ can be assumed to be a simple exponential in line with Beer-Lambert, or even quasi-constant). If the single layer of organic material on glass is irradiated with light at a certain wavelength, we can calculate the exciton generation profile per area $G(x)$ as a function of position in the layer, using the transfer matrix method described before. This profile will typically be a standing wave, as depicted in Figure 2.7. Since no excitons are flowing in or out of the layer, $J_{e}$ will be 0 . Recombination $R(x)$ can be assumed to be a linear function of $\mathrm{e}(\mathrm{x})$ and average carrier lifetime, as in Eq. 2.22.

Eq. 2.31 becomes:

$$
-D_{e} \frac{\delta^{2} e(x)}{\delta x^{2}}=G_{e}(x)-\frac{e(x)}{\tau}
$$

It becomes clear that the working principle of neat organic semiconductor is governed by three main exciton processes competing with each other: photo-generation, recombination and diffusion. Conventionally in literature the boundary conditions of a layer bounded by insulators (glass and air) are taken as $\frac{d e(x)}{d x}=0$, at the edges of the layer $x=0$ and $x=L^{55}$. Solving Eq. 2.32 results in a concentration profile $e(x)$, and the recombination profile can be plotted as $\frac{e(x)}{\tau}$ (see Figure 2.7). The two material properties $\tau$ and $D_{e}$ can be characterized together by one parameter, the diffusion length $L_{d}$

$$
L_{d}=\sqrt{\tau D_{e}}
$$

Which singularly affects how the recombination profile will look. Increasing the diffusion length will result in a more flattened recombination profile. 


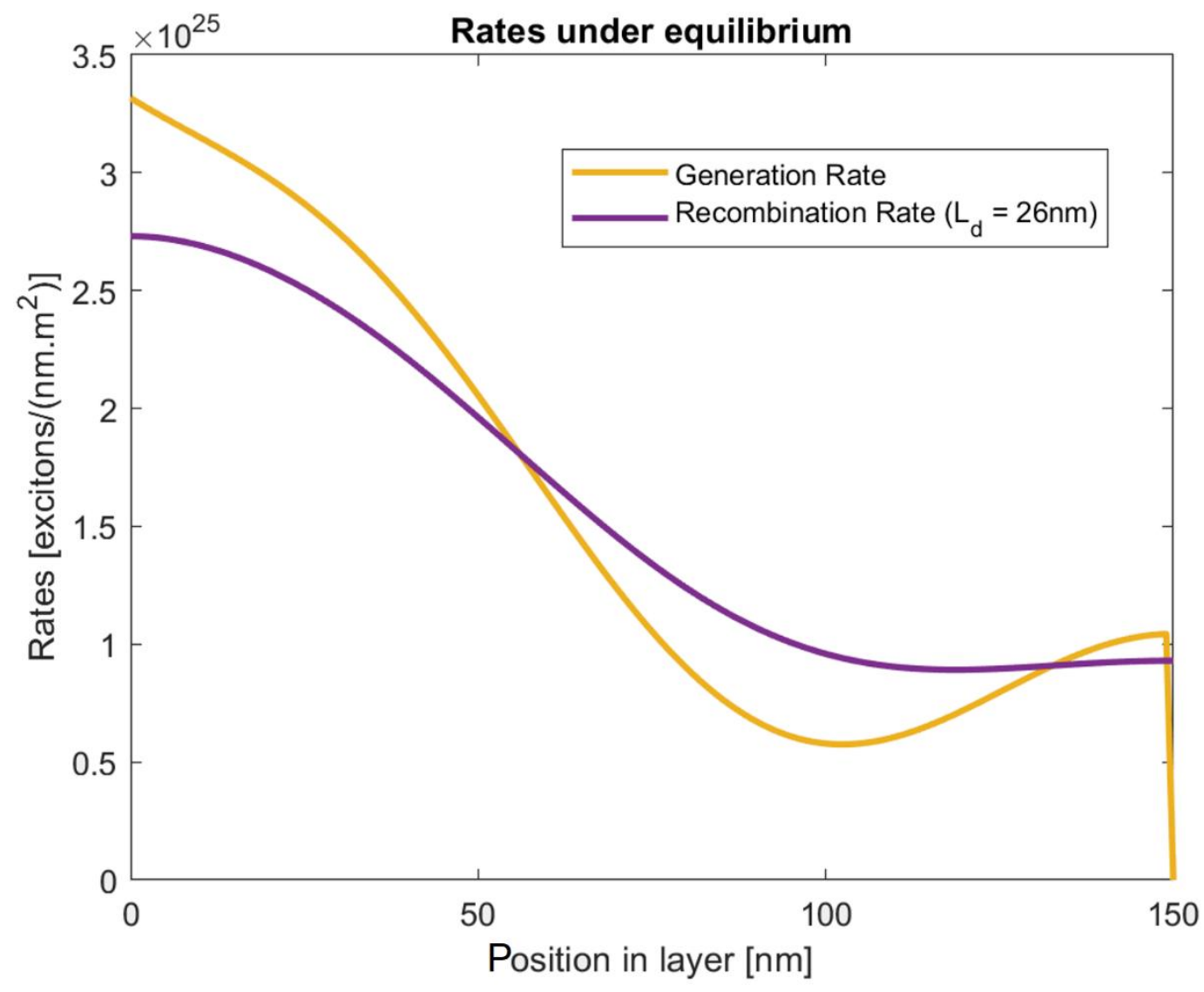

Figure 2.7 Drift Diffusion Example

Figure 2.7. Modelled Generation and Recombination profile in a 150nm PCDTBT layer on glass, under $600 \mathrm{~nm}$ wavelength illumination. Diffusion length is set to around $26 \mathrm{~nm}$ to demonstrate the effect of diffusion.

In most organic semiconductors, the diffusion length is below $10 \mathrm{~nm}$, and the recombination profile will be very close to the generation profile. In organic blends the dynamics naturally change somewhat as there is an exciton dissociation taking place at the donor-acceptor interfaces in the blended materials. This will be discussed more in-depth in Chapter 5.

\subsection{Experimental methods}

Although significant differences between organic and inorganic opto-electronic devices have been discussed, the main phenomena that can drive the efficiency remain unchanged:

- The charge generation efficiency, contained in both EQE and IQE. The key difference in organic semiconductors is that standing waves must be accounted for with transfer matrix modelling, because of the optically thinner layers. 
- The charge collection efficiency, related to electrical recombination effects and characterized by the IQE alone. The key difference for organics here is the existence of excitons, and the charge transfer state as a consequence of using two materials to separate the excitons into free holes and electrons. We note again that in inorganic semiconductors such as c-Si, free carriers are spontaneously created at room temperature since the binding energy is $<k T$ by some margin.

The discussion will now focus on how to characterize these efficiencies experimentally.

\subsubsection{Optical characterization}

The main properties that need to be determined optically are the absorptance, reflectance and transmittance of the device (see Eq. 2.13-2.15) and constituent layers. Reflectance and Transmittance are measured with a spectrophotometer. The most accurate type of measurement is often done with a spectrophotometer that includes an integrating sphere, which is a sphere-shaped chamber that is fully reflective inside and has several detectors, so as to capture all angularly scattered light as well (see Figure 2.8 ).

\section{MM SPHERE}

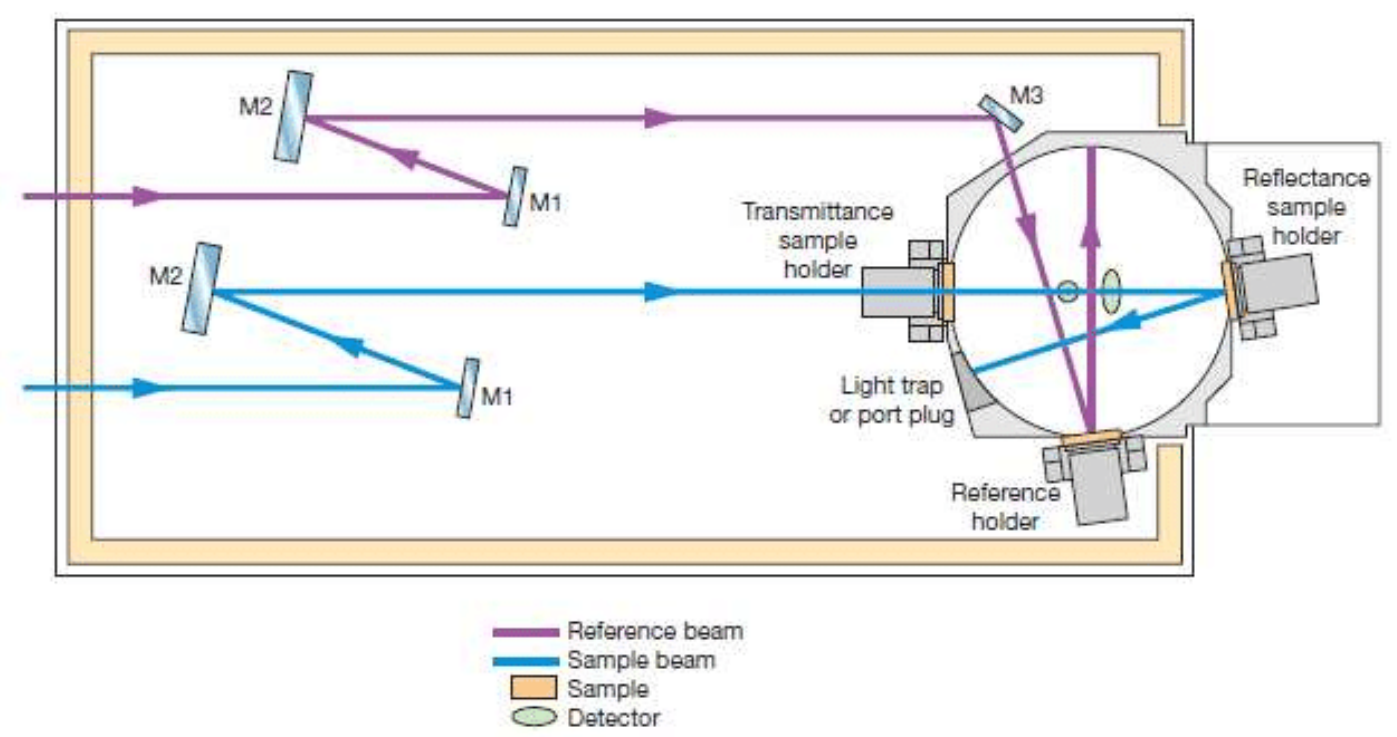

Figure 2.8 Spectrophotometer Diagram

Figure 2.8. Top view of a spectrophotometer diagram with integrating sphere (image modified upon reproduction from Perkin Elmer manual). M1-2 are mirrors, the blue beam is the measurement beam and the purple beam is the reference beam (one source emits both, to reduce noise in a so-called dual-beam configuration). 
For the absorptance, the accurate thicknesses of all layers within the device must be obtained, as well as the optical constants of the materials in all layers. One of the problems is that these are very hard to obtain in the actual, multi-layered device after fabrication, and often every layer will need to be fabricated as a single layer (following the device fabrication conditions as closely as possible) on a substrate of silicon or glass.

Layer thickness can be measured through a variety of techniques, the most common and inexpensive of which is to use a profilometer, in which case a scratch is made in the layer with a thin metal blade, reaching down to the substrate, and a sensitive needle applies a small pressure to the sample and moves to map out the thickness profile of the scratch. However, for very thin layers, below $100 \mathrm{~nm}$, more sophisticated methods are required. These tend to be more resource-intensive and include Atomic Force Microscopy (AFM), X-ray Microscopy (XRM), and ellipsometry. Ellipsometry is often the most useful one since it is also a requirement to derive the second necessary property, the optical constants. This particular method, its limitations and it alternatives, will be discussed in more detail in Chapter 3.

\subsubsection{Electrical characterization}

Once optical constants and layer thicknesses are known a device can be fabricated and the EQE of the device can be measured. EQE measurement has been described before, and usually uses a source meter unit set-up to measure the collected charges. The AM1.5G lamp source is used as reference for the incident amount of photons, and the software converts the measured amount of collected charges to a relative measure in percentage. Usually the $J V$ curves, as shown in Figure 2.2, are also measured using an oscilloscope set-up. The actual fabrication of particular devices will be expanded upon in later chapters. It will however be useful to consider what to do with the EQE data, and what else we need to know when IQE has been determined through optical analysis and Eq. 2.15.

In order to really understand the IQE we need to characterize the recombination going on in the active materials of the device. While there are many methods to do this, such as Transient Photocurrent (TPC), Time-delayed Collection Field (TDCF) and charge extraction by linearly increasing voltage (CELIV) a popular method that will be examined more closely in this work is the measuring the emission or luminescence of the material. In the case of Photoluminescence (PL), emission is stimulated through incident photons that are within 
the absorption regime of the active material. The emitted light from the recombination will be of much lower intensity and Stokes shifted ${ }^{56}$, meaning the emission will be at a slightly longer wavelength (lower energy) than the incident light. A filter is therefore applied to stop the incident light (which will not be fully absorbed) from being detected. A possible set-up for the experiment is shown in Figure 2.9.

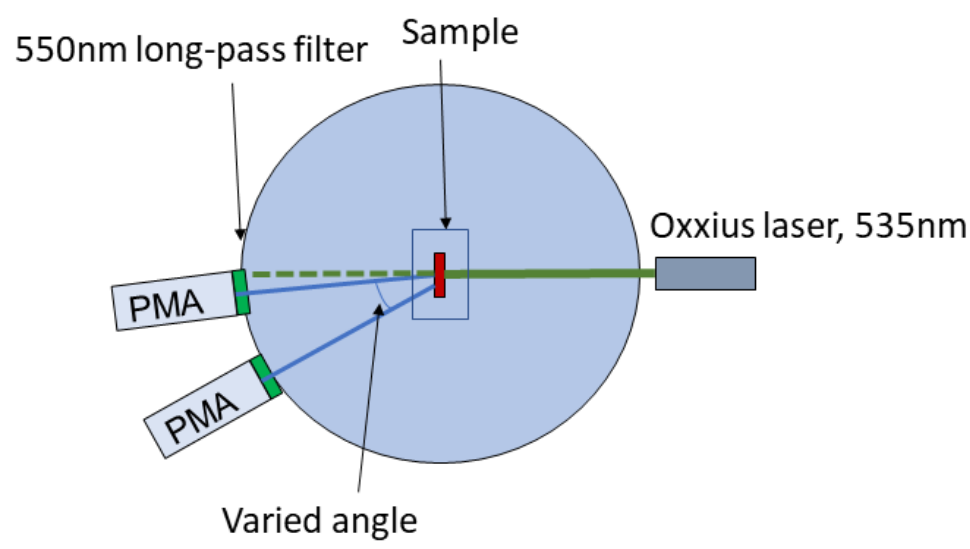

Figure 2.9 Rotating Emission Set-Up

Figure 2.9. Rotating set-up for measuring photoluminescence on an organic sample on glass. A Photonic Multichannel Analyzer (PMA-12) is used to detect the relative amount of emitted photons at a certain angle, and can be rotated around the stationary sample.

For Electroluminescence (EL), the charge carriers are injected into the device electrically instead, after which they recombine and emit. EL requires electrical contacts to be measured, but while PL seems simpler it comes with challenges of its own, most notably when trying to detect emission from a CT state rather than from the main singlet state, because photoemission can be very weak and therefore challenging to detect. Once an emission spectrum is measured, it can then be used to derive insight into the available energetic levels (or rather the difference in levels) within the device for free carriers.

There are also different set-ups depending on whether transient or steady-state luminescence is being measured. Transient photoluminescence (tr-PL) measurements allow one to derive the time-dependent behaviour and recombination lifetime of the material, in contrast to the steady-state measurements which are more commonly used to analyse the spectrum and from this determine the energetic wavelengths that have the highest emission. Steady state would be used to reveal the energy at which the CT state exists in blend 
materials, while transient measurement would be necessary to determine the lifetime $\tau$ featured in the drift-diffusion equations (Eq. 2.20).

It should be noted that even in these emission measurements, there will again be a distortion caused by the cavity effects that are present in devices with optically thin active layers ${ }^{57}$, in other words for most organic or hybrid devices. Again, this will all be expanded upon more in-depth in chapter 5.

\subsection{Conclusion}

A theoretical framework has been provided explaining the detailed balance theory and its application to opto-electrical devices. This framework was then used to define key concepts such as external and internal quantum efficiency, which were both characterized in terms of optical and electrical considerations, involving such concepts as reflectance and transmittance, the solar spectrum, and the current continuity equations.

This was then extrapolated to second-generation, organic semiconductor materials. Optically these materials pose a challenge due to their low mobility and thin layers, which requires treatment with methods such as the transfer matrix. Electrically these materials are complicated due to their high exciton dissociation energy, which requires blended active layers to facilitate this separation. The charge transfer state and its effect on mobility and quantum efficiency were also discussed.

Finally, some key characterization methods were defined for experimentally investigating novel materials. These methods will now be looked at more in-depth and improved upon for novel organic and hybrid perovskite semiconductor materials in the next chapters, where we will apply the theoretical framework outlined here. 


\section{Determination of optical constants of simple and composite thin films}

The determination of optical constants is a key aspect of the characterization of electro-optical devices and their materials. This chapter will look at how these optical constants are defined today, and how they are determined for thin films. The conventional method of spectroscopic ellipsometry will be evaluated and discussed in the context of composite films. An alternative transmittance method will be discussed and applied to high-index materials such as nonfullerenes and perovskites.

This chapter is substantially based on the author's work published in Advanced Optical Materials $^{58}$

\subsection{Definition of optical constants}

Optical constants fundamentally characterize the apparent speed at which a light waves propagates through a certain medium. Light always travels at a fixed speed $c$, in the sense that information or a light pulse is always conveyed at $c$, regardless of if it travels in vacuum or a medium ${ }^{59}$. However, when considering light as an electromagnetic wave as proposed by Maxwell, the phase velocity $v_{p}$ at which the wave appears to propagate will deviate from $c$, depending on the medium it travels in. This apparent wave speed $v_{p}$ at a frequency $f$ is given by:

$$
v_{p}=\lambda_{m} \cdot f
$$

where the medium wavelength $\lambda_{m}$ is an apparent wavelength. It is altered from the vacuum wavelength due to the displacement response of the bound electrons in the medium to the propagating wave. It is linked to the original wavelength in vacuum as following

$$
\lambda_{m}=\frac{\lambda}{n(\omega)}
$$

where $n(\omega)$ is called the refractive index of the medium. Note that this refractive index depends on the radial light frequency $\omega$. As a consequence, the apparent phase velocity of the light wave in the medium can also be written as: 


$$
v_{p}=\frac{c}{n(\omega)}
$$

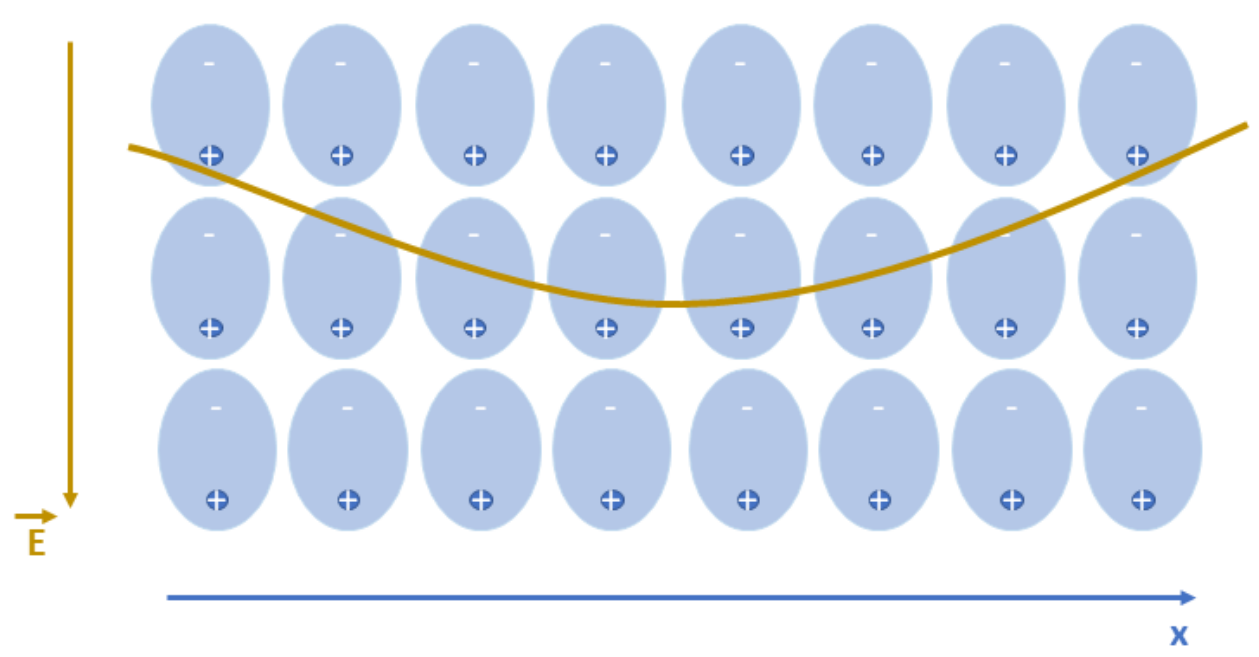

Figure 3.1 Dipole Model

Figure 3.1. A simplified model of polarized dipoles, consisting of a negative electron cloud and positive nucleus, under influence of an oscillating electric field $\boldsymbol{E} e^{i \omega t}$. When treated with a Lorentzian model, the dipoles will be oscillating at the same frequency $r e^{i \omega t}$, resulting in a susceptibility $\chi_{\mathrm{e}}$ that is only frequency-dependent (see Eq. 3.4).

Depending on the configuration of the electron orbitals in the material, the electrons will be more or less responsive to the traversing electromagnetic wave, a response which is characterized by the dielectric permittivity $\varepsilon(\omega)$ and magnetic permeability $\mu(\omega)$ of the material. Dipoles, magnetic or electric, will be induced or aligned in the material when an external electric or magnetic field is applied, and these dipoles will orient themselves in response to the external field. $\varepsilon(\omega)$ and $\mu(\omega)$ characterize this polarization of dipoles on a macroscopic scale. The practical significance of these constants will be demonstrated with the derivation of the dielectric permittivity $\varepsilon$. The derivation of $\mu$ is somewhat analogous but since the deviation from vacuum permeability is often insignificant it is usually assumed to be $\mu_{0}$, the vacuum value.

If we define a polarization $P$ as the average electric dipole moment per unit volume of the medium, then for most materials the relation between $P$ and the external electric field $E$ is linear, and can be given as:

$$
\boldsymbol{P}=\varepsilon_{0} \chi_{\mathrm{e}}(\omega) \boldsymbol{E}
$$


where $\chi_{\mathrm{e}}(\omega)$ is called the electric susceptibility and $\varepsilon_{0}$ is the permittivity in vacuum. Note that the susceptibility depends on the light frequency $\omega$, because while for a static system the dipole moment simply opposes the external field, for a time-dependent light wave the dipole response is more like that of a damped harmonic oscillator. We define an electric displacement field $D$ :

$$
\boldsymbol{D}=\varepsilon_{0} \boldsymbol{E}+\boldsymbol{P}
$$

This displacement field definition allows Gauss' Law to be described in terms of only the free charge density in the medium, $\rho_{\text {free }}$, and allows us to implicitly account for the bound charge density, $\rho_{\text {bound }}$. Gauss' law becomes:

$$
\begin{array}{r}
\Delta \cdot \boldsymbol{E}=\frac{\rho_{\text {free }}+\rho_{\text {bound }}}{\varepsilon_{0}} \\
\Delta \cdot \boldsymbol{E}=\frac{\rho_{\text {free }}-\Delta \cdot \boldsymbol{P}}{\varepsilon_{0}} \\
\Delta \cdot \boldsymbol{D}=\rho_{\text {free }}
\end{array}
$$

Similarly, Ampere's Law in a magnetically susceptible medium can be written in terms of just the free current density $J_{\text {free }}$ and a magnetizing field $\mathrm{H}$, which similarly already contains bound charge:

$$
\Delta \times \boldsymbol{H}=J_{\text {free }}
$$

Both these newly defined fields (displacement field $D$ and magnetizing field $H$ ) can be written in terms of the original electric and magnetic fields, $E$ and $B$, as following:

$$
\begin{aligned}
\boldsymbol{E} & =\varepsilon \boldsymbol{D} \\
\boldsymbol{B} & =\mu \boldsymbol{H}
\end{aligned}
$$

Inspection of Eq. 3.4 and 3.5 shows a necessary relation between the material permittivity $\varepsilon$ and the electric susceptibility $\chi_{\mathrm{e}}$, and similar relations are reached for $\mu$ and the magnetic susceptibility $\chi_{\mathrm{m}}$ :

$$
\begin{aligned}
& \varepsilon(\omega)=\varepsilon_{0}\left(1+\chi_{\mathrm{e}}(\omega)\right) \\
& \mu(\omega)=\mu_{0}\left(1+\chi_{\mathrm{m}}(\omega)\right)
\end{aligned}
$$


These are already called 'optical constants' and can further be linked to the refractive index $n(\omega)$ of the material, using Maxwell's equations. Maxwell's equations for an (infinite) nonvacuum medium can be written in a simplified manner that implicitly already contains the bound charge, using the earlier defined fields $D$ and $H$. In the absence of any free charge or currents in the material, Maxwell's equations are written as:

$$
\begin{array}{lll}
\Delta \cdot \boldsymbol{D}=0 & \text { and } & \Delta \times \boldsymbol{H}=\frac{\delta \boldsymbol{D}}{\delta t} \\
\Delta \cdot \boldsymbol{B}=0 & \text { and } & \Delta \times \boldsymbol{E}=\frac{\delta \boldsymbol{B}}{\delta t}
\end{array}
$$

These equations have the same form as the Maxwell equations in vacuum, and can be solved to yield a similar plane wave solution, with the exception that the plane waves of the solution now travel at a different phase speed $v_{p}$, owing to our substitution of the new fields using Eq. 8:

$$
\begin{gathered}
\overrightarrow{\boldsymbol{E}(\mathbf{z}, \boldsymbol{t})}=\boldsymbol{E} e^{i\left(\frac{2 \pi}{\lambda} \sqrt{\varepsilon \mu} \cdot z+\omega t\right)}=\boldsymbol{E} e^{i(k \cdot z+\omega t)} \\
v_{p}=\frac{\omega}{k}=\frac{1}{\sqrt{\varepsilon \mu}}
\end{gathered}
$$

Therefore, using the definition of refractive index in Eqs. 3.2-3.3, the relation with the refractive index can now be shown to be:

$$
n(\omega)=c \sqrt{\varepsilon(\omega) \mu(\omega)}
$$

Or, using the fact that $\mathrm{c}=\frac{1}{\sqrt{\varepsilon_{0} \mu_{0}}}$

$$
n(\omega)=\sqrt{\frac{\varepsilon(\omega) \mu(\omega)}{\varepsilon_{0} \mu_{0}}}=\sqrt{\varepsilon_{r}(\omega) \mu_{r}(\omega)}
$$

Where $\varepsilon_{r}$ and $\mu_{r}$ are called the relative permittivity and permeability. As mentioned before, the optical constants of a material are often linked to only the dielectric part while the relative magnetic permeability $\mu_{r}$ is assumed to be close to 1 .

This electronic response will also give rise to different optical effects such as refraction, reflection at boundaries, and altered interference due to the modified wavelength and various reflections. This is why knowledge of these optical constants is crucial in describing any opto-electrical device. 
The electronic response to impinging light will be transitory, as long as the examined medium is transparent and doesn't absorb any photons, that is, if the light is not of the right frequency (or energy) for the electrons to reach new, stable orbital.. However, if the material does absorb, the electrons will be propelled to a higher, stable energetic state and the EM wave will decrease in amplitude as it passes through the material. This behaviour can be described mathematically by assuming a complex part to the refractive index. If we consider the timeindependent part of the plane wave function, it can be written as:

$$
\begin{gathered}
\overrightarrow{E(z)}=\boldsymbol{E} e^{i \frac{2 \pi}{\lambda} \bar{n} \cdot z}=\boldsymbol{E} e^{i \frac{2 \pi}{\lambda}\left(n_{r}+i n_{i}\right) \cdot z} \\
=\boldsymbol{E} e^{i \frac{2 \pi n_{r}}{\lambda} z} \cdot e^{\frac{-2 \pi n_{i}}{\lambda} z}
\end{gathered}
$$

Since the imaginary part of the exponential can be described in terms of a real sine and complex cosine, this represents the part of the wave that is not absorbed. The attenuation is entirely characterized by the real part of the exponential, shown in red, which involves the imaginary part of the refractive index, $n_{i}$. This imaginary part $n_{i}$ is often simply denoted as $k$, a convention that will be used from here on out. In other words, the full complex refractive index is from here on denoted as $\bar{n}$, the real part as $\eta$, and the imaginary part as $k$ :

$$
\bar{n}(\omega)=\eta(\omega)+i k(\omega)
$$

and similarly for the dielectric constants to which they are related:

$$
\bar{\varepsilon}(\omega)=\varepsilon_{r e}(\omega)+i \varepsilon_{i m}(\omega)
$$

Assuming a semi-infinite medium, we can then recover the Beer-Lambert law (Eq. 2.16) of absorption in terms of this imaginary optical constant $k$, as well as redefine the absorption coefficient $\alpha$ and the absorptance $A$. The intensity (or power per incident area) of the light beam $I(z)$ can be shown to be proportional with the square of the electric field $E(z)$, according to the Poynting theorem ${ }^{60}$ :

$$
I(z)=\frac{c \eta \varepsilon_{0}}{2}|E(z)|^{2}
$$

The absorption coefficient (also more generally called attenuation coefficient) is defined as:

$$
\alpha(z)=-\frac{d I(z) / d z}{I(z)}
$$


which can now be calculated, using Eq. 3.15 and 3.17, to equal

$$
\alpha=\frac{4 \pi k}{\lambda}
$$

for the simple plane wave. In other words, it is independent of position in the medium. This means that the introduction of a complex refractive index was justified, since it offers a useful description in terms of absorption. In the case of significant reflection (for thinner layers), $I(z)$ will no longer be a simple plane wave but instead a sum of two plane waves, one traveling forwards and one travelling backwards, with their amplitude depending on position $z$ within the layer. In that case Eq. 3.15 (which implies Beer-Lambert) will no longer be valid and the transfer matrix approach will need to be used to calculate $l(z)$.

The absorptance $A$ is defined as the following ratio:

$$
A=\frac{I_{\text {absorbed }}}{I_{\text {incident }}}
$$

which can be described in terms of the absorption coefficient as well, since $I_{\text {absorbed }}=$ $\int_{0}^{L} d I(z) / d z$, with $L$ denoting the thickness of the absorbing layer:

$$
A=\frac{\int_{0}^{L} \alpha(z) I(z)}{I_{\text {incident }}}
$$

Since in the transfer matrix we know the total forwards- and backwards-propagating field at every layer interface, we can calculate the Electric field within the stack at every position $z$, $\mathrm{E}(\mathrm{z})$, and use this to calculate the absorptance per layer $\mathrm{i}, A_{i}$ :

$$
A_{i}=\frac{\int_{0}^{L} \alpha_{i} \frac{c \eta \varepsilon_{0}}{2}\left|E_{i}(z)\right|^{2}}{\frac{c \varepsilon_{0}}{2}\left|E_{0}\right|^{2}}
$$

This same equation can be found in papers conventionally used as instruction for transfer matrix modelling of multilayer absorption, such as the work by Pettersson et al ${ }^{61}$.

\subsection{The Kramers-Kronig relations}

It is important to note that there is a direct relation between the imaginary part and the real part of the dielectric constants (and of the refractive indices), which have been previously 
defined in Eq. 3.16. This can be derived by examining the relation between the inducing field and the electric polarization of the molecules of the medium, given by Eq. 3.4:

$$
\boldsymbol{P}=\varepsilon_{0} \chi_{\mathrm{e}}(\omega) \boldsymbol{E}
$$

Note that this relation is defined in the frequency domain, rather than the time domain. However there is also a causal relation between the polarization and the inducing electric field, and this can best be given in the time domain. Using Fourier transformation, a timedomain relation for Eq. 3.4 is obtained:

$$
\boldsymbol{P}(t)=\varepsilon_{0} \int_{-\infty}^{t} \chi_{\mathrm{e}}(t-T) \boldsymbol{E}(T) d T
$$

Here, the response function $\chi_{\mathrm{e}}(\Delta t)$ determines the polarization induced by an applied field after a time $\Delta t$. To ensure the causality of $\chi_{\mathrm{e}}(\Delta \mathrm{t})$, it has to be zero for any time $\Delta t<0$. This can be achieved by requiring:

$$
\begin{gathered}
\chi_{\mathrm{e}}(\Delta t)=\chi_{\mathrm{e}}(\Delta t) \vartheta(\Delta t) \\
\vartheta(\Delta t)=0(\Delta t<0) \\
\vartheta(\Delta t)=1(\Delta t \geq 0)
\end{gathered}
$$

The Fourier transform of this special function $\vartheta$ is:

$$
F T\{\vartheta(\Delta t)\}=\frac{\delta(\omega)}{2}+\frac{i}{2 \pi \omega}
$$

And through the convolution theorem the Fourier transform of Eq. 3.24 can now be rewritten as:

$$
\begin{aligned}
\chi_{\mathrm{e}}(\omega) & =\int_{-\infty}^{\infty} \chi_{\mathrm{e}}(\Omega)\left(\frac{\delta(\omega-\Omega)}{2}+\frac{i}{2 \pi(\omega-\Omega)}\right) d \Omega \\
& =\frac{\chi_{\mathrm{e}}(\omega)}{2}+\frac{i}{2 \pi} \int_{-\infty}^{\infty} \frac{\chi_{\mathrm{e}}(\Omega)}{\omega-\Omega} d \Omega
\end{aligned}
$$

Rearranging this in terms of $\chi_{\mathrm{e}}(\omega)$ gives:

$$
\chi_{\mathrm{e}}(\omega)=\frac{i}{\pi} \int_{-\infty}^{\infty} \frac{\chi_{\mathrm{e}}(\Omega)}{\omega-\Omega} d \Omega
$$


Recalling the relation between $\chi_{\mathrm{e}}(\omega)$ and the dielectric constant in Eq. 3.9a, and rearranging the denominator, we obtain:

$$
\begin{gathered}
\varepsilon_{r e}(\omega)=1+\frac{1}{\pi} \int_{-\infty}^{\infty} \frac{\varepsilon_{i m}(\Omega)}{\Omega-\omega} d \Omega \\
\varepsilon_{i m}(\omega)=-\frac{1}{\pi} \int_{-\infty}^{\infty} \frac{\varepsilon_{r e}(\Omega)}{\Omega-\omega} d \Omega
\end{gathered}
$$

These are the famous Kramers-Kronig dispersion relations, giving the relation between the real and imaginary parts of the dielectric constants. From here, the relation between the real and imaginary refractive index can also be obtained:

$$
\begin{aligned}
& \eta(\omega)=1-\frac{c}{2 \pi \omega} \int_{-\infty}^{\infty} \frac{\alpha(\Omega)}{\Omega-\omega} d \Omega \\
& \alpha(\omega)=\frac{2 \omega}{c \pi} \int_{-\infty}^{\infty} \frac{\eta(\Omega)-1}{\Omega-\omega} d \Omega
\end{aligned}
$$

Where $\alpha(\omega)$ is the absorption coefficient and still equals $\frac{4 \pi k(\omega)}{\lambda}$ (this is true even for reflected plane waves in a multi-layer stack since the field can be treated as the sum of a forwards- and backwards-propagating plane wave, each of which will decrease in amplitude in the same fashion as a normal plane wave, just in opposite direction). An even more convenient form can be obtained in terms of wavelength rather than frequency, since the transfer matrix protocol is often expressed in terms of position (and therefore wavelength) as well:

$$
\begin{gathered}
\eta(\lambda)=1+4 c \oint_{0}^{\infty} \frac{\frac{2 \pi c}{\Lambda^{3}} \cdot k(\Lambda)}{\left(\frac{2 \pi c}{\Lambda}\right)^{2}-\left(\frac{2 \pi c}{\lambda}\right)^{2}} d \Lambda \\
k(\lambda)=d \Lambda
\end{gathered}
$$

These relations will play a vital role in experimental methods for determining the optical constants of novel opto-electric materials. A flowchart detailing the computational implementation of the relations in Matlab is given below. 


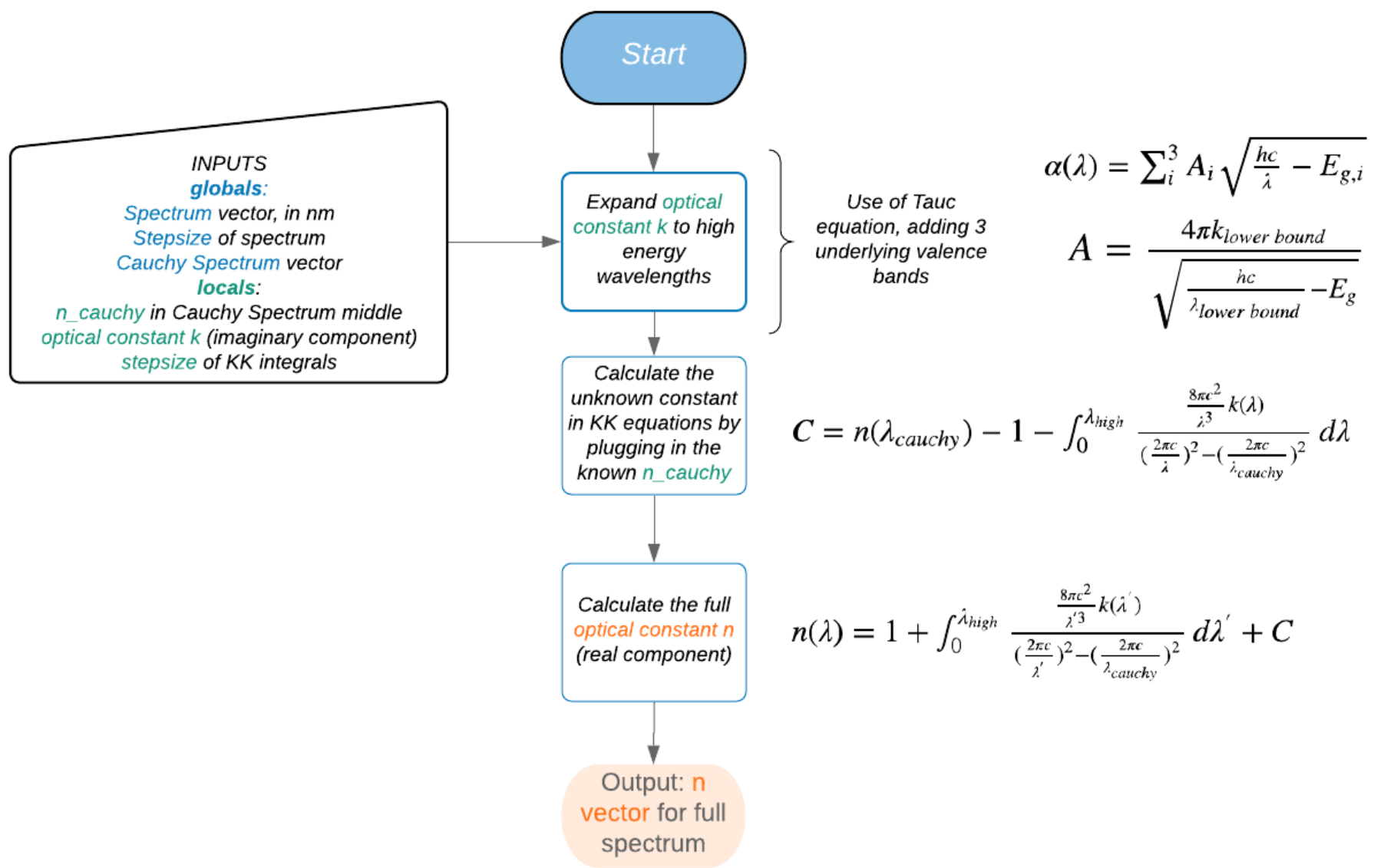

Flowchart 3A: Matlab Kramers-Kronig procedure (KK.m)

It should be noted that since the procedure involves solving an integral that runs to $\Lambda=0$, an approximation must be made towards this "infinite-energy" point for $k(\Lambda)$, which is unknown. Practically, $k(\Lambda)$ tends to be known down to a wavelength of $300 \mathrm{~nm}$, after which absorption data becomes more difficult to obtain, due to glass substrate absorption past this point. Because of this, the whole $\eta(\lambda)$ function is shifted by a constant to be determined from a known $\eta(\lambda)$ data point in the Cauchy regime, similarly to if we were solving an indefinite integral. Furthermore, the known $k(\Lambda)$ data can be extended using some basic approximations from the Tauc model of absorption, as outlined in Flowchart 3A. The particular formula used in the Matlab script is:

$$
\left(\alpha_{i j}(\Lambda) h v\right)^{1 / n}=A_{i j}\left(h v-E_{g, i j}\right)
$$


Where $E_{g, i j}$ are the examined band gaps (or MO energetic off-sets), $A_{i j}$ a proportionality constant determined from known data, and $\alpha_{i j}(\Lambda)$ the absorption coefficient for each band gap at a certain wavelength. The total absorption coefficient is then simply the sum of $\alpha_{i j}(\Lambda)$ and $k(\Lambda)$ can be determined using Eq. 3.19. For the script, the modelled band gaps are the primary band gap that sets in with absorption, and 2 additional ones at $200 \mathrm{~nm}$ and $100 \mathrm{~nm}$. While this is a very crude approximation, the main reason for this extension is to obtain reasonable values close to $300 \mathrm{~nm}$. The further away from this wavelength, the less influence the extension actually has (this can be seen in Figure 3.2).

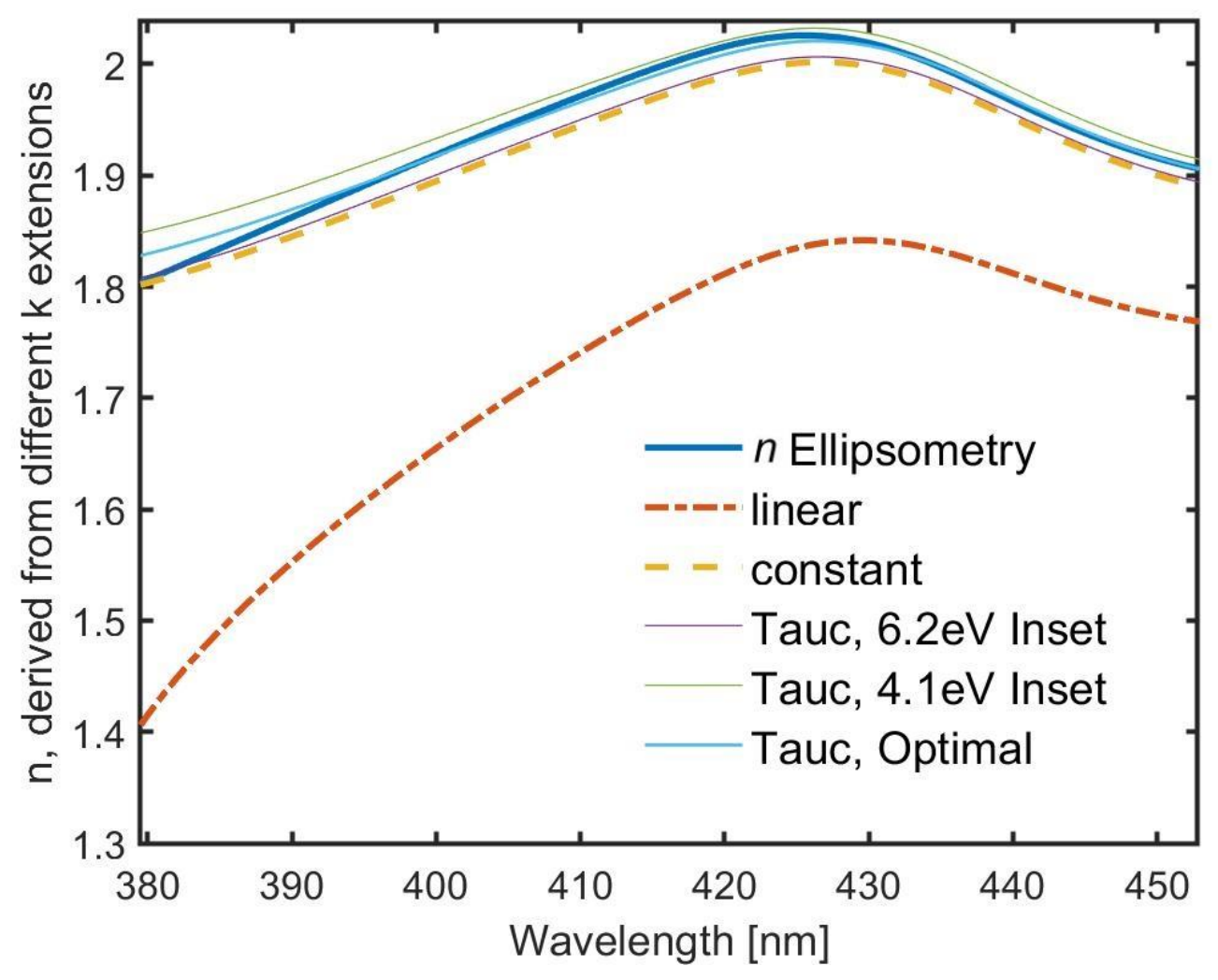

Figure 3.2 Kramers Kronig Extension

Figure 3.2: Effect of various models for a Kramers Kronig extension towards the high-energy regime. As can be seen, the difference between Tauc models with different parameters is quite small, on the order of a relative error of up to $0.3 \%$. 


\subsection{Ellipsometry}

\subsubsection{Procedure}

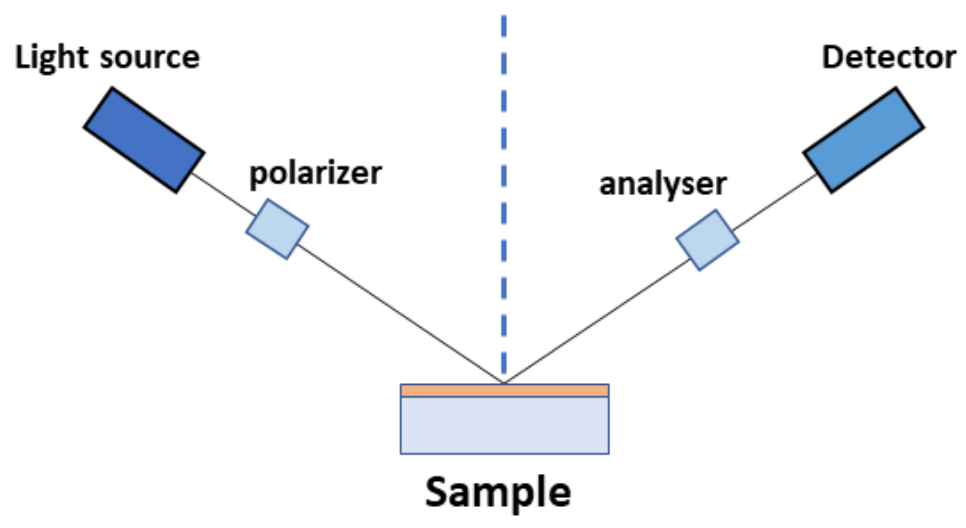

Figure 3.3 Ellipsometer Set-Up

Figure 3.3 Diagram of an ellipsometer set up. The incident beam is linearly polarized and then reflected, after which an analyse polarizes the light again before detection of the respective reflected, polarized light amplitudes.

It is time to look at some more in-depth and extensive uses of the method to characterize advanced single layers. Ellipsometry works through a polarization-controlled incident beam which is impinged upon the sample under a certain angle (usually close to the Brewster angle) and reflected off the sample surface (see Figure 3.3). The differently polarized amplitudes of the incident beam are reflected differently and the amplitudes of the $s$ and $p$ polarizations, $A_{s}$ and $A_{p}$, are measured. From these we can determine the amplitude component $\psi$ and phase difference $\Delta$ :

$$
\frac{A_{p}}{A_{s}}=\tan (\psi) e^{-\Delta}
$$

Both $\psi$ and $\Delta$ are then fitted for three separate angles to introduce enough redundancy for the fit, and from this fit the optical constants of the material can be derived, after a thin-film on glass measurement. The software used for this fitting was CompleteEASE, provided by J.A. Woollam. An excellent reference work on the details of this software is also provided ${ }^{62}$. The general procedure with the software is to select the Cauchy Regime region, that is the region where no absorption in the material occurs due to the bandgap (or for organics, the HOMOLUMO difference) being of a higher energy than the incident light's wavelength. Generally, 
this region can be found by plotting $\Delta$ against the frequency domain and looking for a region without break of periodicity and or continuity, as demonstrated in figure 3.4.

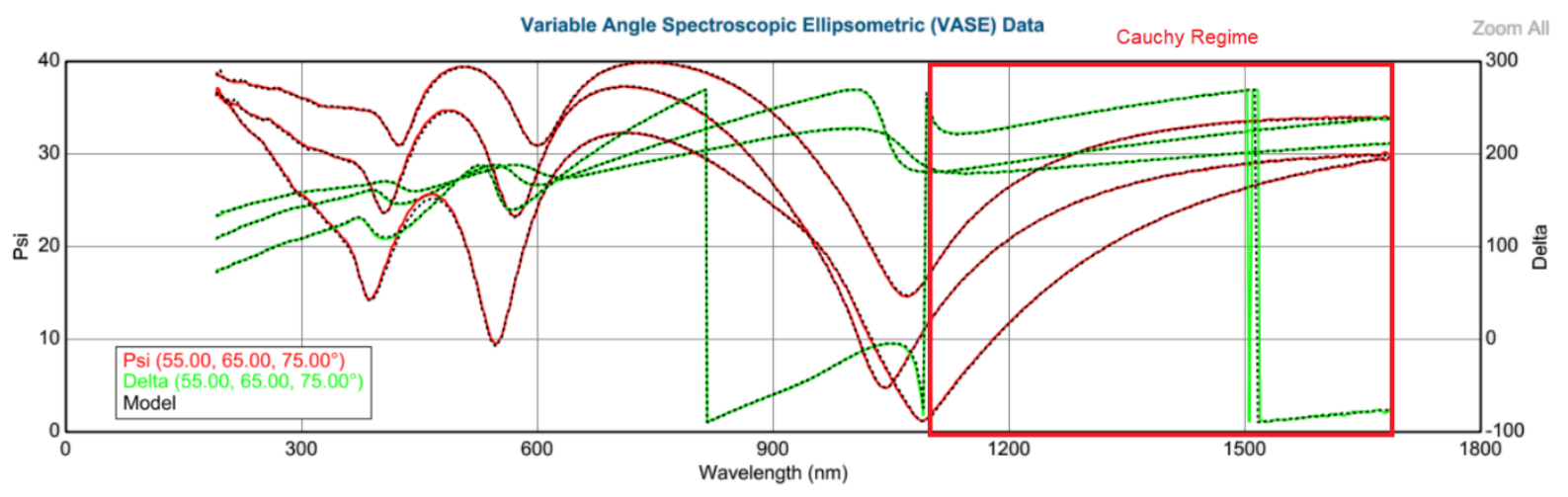

Figure 3.4. Ellipsometer Fit Graph

Figure 3.4: Example of psi $(\psi)$, delta $(\Delta)$ diagram, modified upon reproduction from the CompleteEase software manual. Notice the outline of Cauchy region, which can be recognized by its continuity in Delta.

A Cauchy model is then fitted to the data here, which means the imaginary part of the refractive index, $k$, is assumed 0 here, while $\eta$ is assumed to follow a Cauchy expansion as a function of wavelength:

$$
\eta(\lambda)=A+\frac{B}{\lambda^{2}}+\frac{C}{\lambda^{4}}+\cdots
$$

This is an empirical formula only applicable for the Cauchy regime and is usually fitted only up to free variable $C$. The thickness of the layer is also allowed as a free variable during this fit, and if a good starting thickness is chosen (this can be done by visibly comparing model and data), it will converge to a fit with a small MSE (mean squared error). Once a good fit is obtained, a mathematical B-spline fitting model is substituted for the Cauchy model, because the B-spline model also allows for a non-zero $k(\lambda)$. This B-spline model is then extrapolated to fit the wavelengths outside the Cauchy regime, refitting every time as data from absorbing wavelengths is added to the model. During this time the previously found layer thickness is kept fixed, while both $\eta(\lambda)$ and $k(\lambda)$ are fitted.

This procedure generally yields a fairly accurate set of optical constants for the single layer material. It is also possible to retroactively impose the Kramers-Kronig relation on the fit after 
it has already converged to a close fit, but usually this will not have a big impact and sometimes this can lead to erroneous results.

This procedure works well and can yield very accurate $\eta, k$ and layer thickness for most materials. However, for third-generation materials there are some complications.

\subsubsection{Effective medium theory}

One such a complication is the use of porous layers, consisting of two different materials in one layer. This type of structure is now commonly used for perovskite devices ${ }^{63,64}$, based on the earlier work with dye-sensitized optical devices ${ }^{65}$. The reason a porous substrate layer is used will be expanded on in chapter 4 when the full device is discussed. More relevant to this chapter, to characterize such a layer consisting of 2 materials with ellipsometry requires certain adjustments to the fitting model.

These adjustments are based on Bruggeman effective medium theory. Stated concisely, the porous combination of materials can be modelled as one material by applying a statistical volume average on the microscopic scale when looking at electric field and induced polarizability, and from here deriving an effective dielectric constant (or refractive index). There are several approaches but the one used in CompleteEASE is the Bruggeman approach $^{66}$, given at each wavelength $\lambda$ by:

$$
f_{a} \frac{\varepsilon_{a}-\varepsilon}{\varepsilon_{a}+2 \varepsilon}=-f_{b} \frac{\varepsilon_{b}-\varepsilon}{\varepsilon_{b}+2 \varepsilon}
$$

Where $\varepsilon_{a}$ and $\varepsilon_{b}$ are the dielectric constants of the pure phases a and $\mathrm{b}, f_{a / b}$ represents the respective volume fractions of the phases, and $\varepsilon$ is the (complex) effective dielectric constant (the effective refractive index $\bar{n}(\lambda)$ can then be derived using Eq. 3.13).

Because this introduces several new free parameters in the fitting process, a more extensive experimental measurement needs to be performed to ensure there is enough data to converge to a meaningful fit. One technique that satisfies this is dynamic porosymmetry: the porous thin film sample on glass is dynamically infiltrated with a liquid of known refractive index like toluene, while performing ellipsometry on the sample. Since both the refractive indices of air and toluene are known, and only the porous material (for example, mesoporous Titania) is unknown, the volumetric fraction $f_{a}\left(=1-f_{b}\right)$ can then be fitted for. Once this is known, ellipsometry can be performed on the pure material that will be infiltrated in the 
porous material for the device layer (for example, perovskite), and the effective refractive index of the mixed materials single porous layer can be determined using Eq. 3.33.

While this process arrives at a satisfactory set of optical constants for third-generation materials, the measurement process is laborious and expensive. Moreover, for the basic procedure there are additional problems with perovskites and non-fullerenes, caused by the high real components of the refractive indices of these materials. In this case the difference between reflected s- and p-polarized light becomes too small/large to obtain a good fit with ease. Moreover, the required equipment for ellipsometry measurements can be costly and difficult to master. For the purpose of addressing these challenges, an alternative method was developed to determine optical constants.

\subsection{Transmittance method}

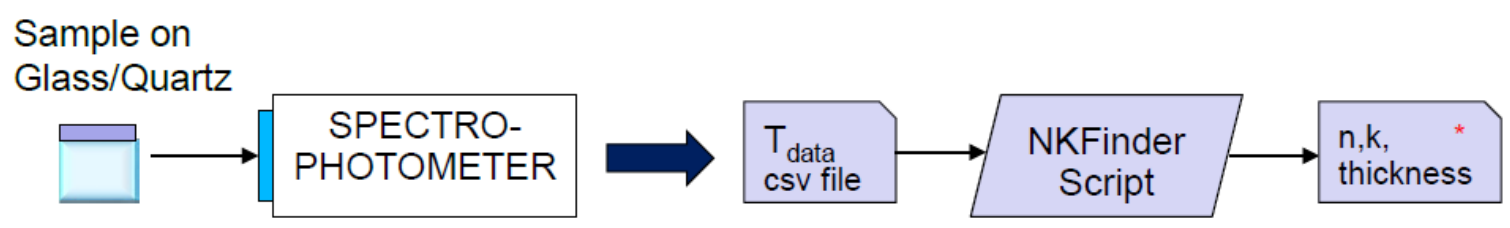

Figure 3.5 Transmittance Method Overview

Figure 3.5: A general overview of the transmittance / transfer matrix procedure when modelling a single or multi-layer stack with unknown optical constants.

The transmittance method is an alternative methodology to obtain optical constants. It requires two simple transmittance measurements on two thin-film samples on glass of the examined material, each sample of a slightly different thickness. Similar methodologies existed in the literature before, but these were often limited by requiring another variable, such as reflectance measurements, a type of measurement that is noted for its difficulty to obtain accurate results ${ }^{67}$.

Using a Perkin Elmer spectrophotometer, transmittance measurements of a range of organic and hybrid organic-inorganic thin-film samples on glass were obtained. Flowcharts $3 \mathrm{~B}$ and $3 \mathrm{C}$ below describe the Matlab procedure for the subsequent analysis. 


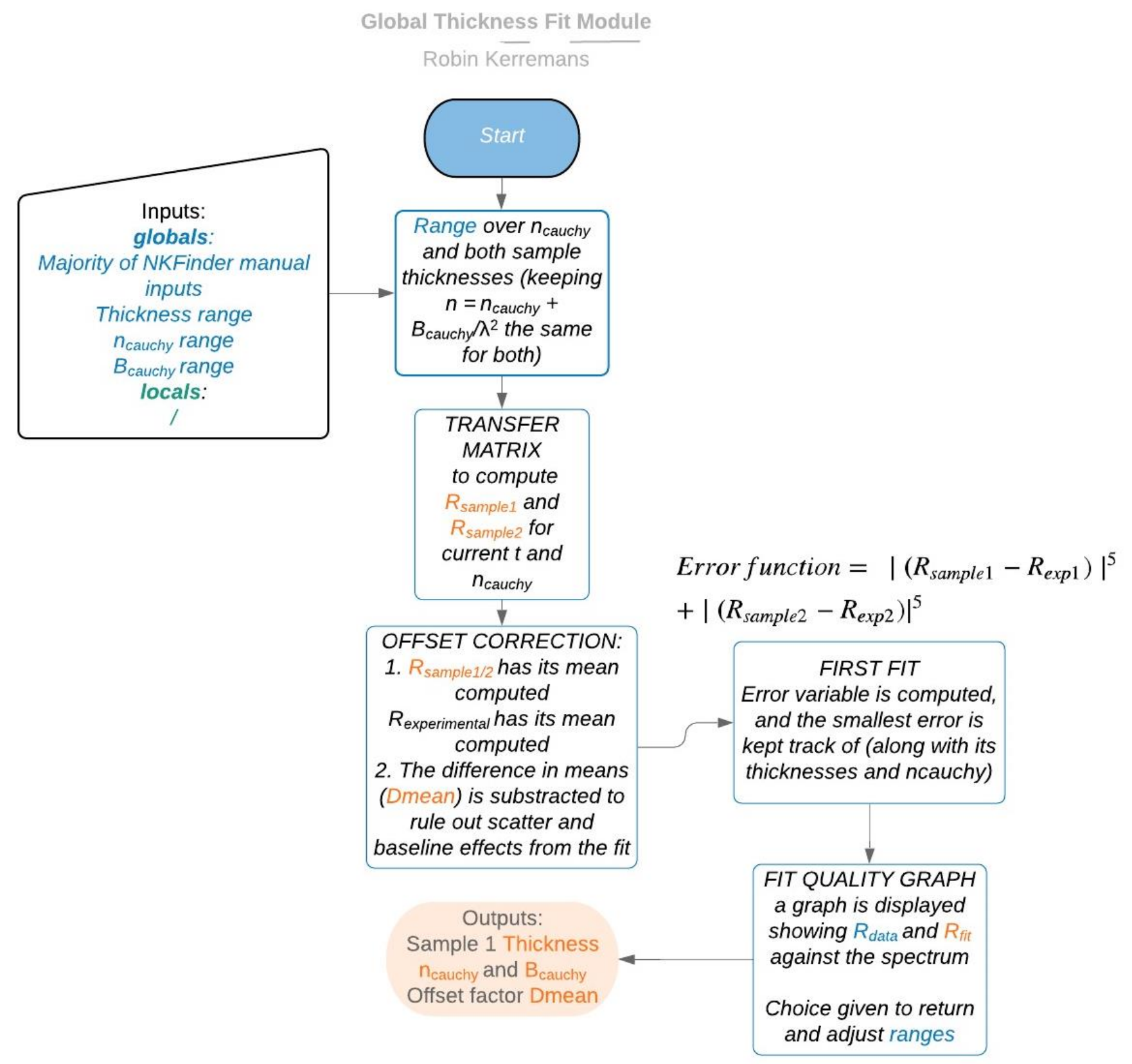

Flowchart 3B: Matab transmittance method thickness finder script (GT_Finder.m) 


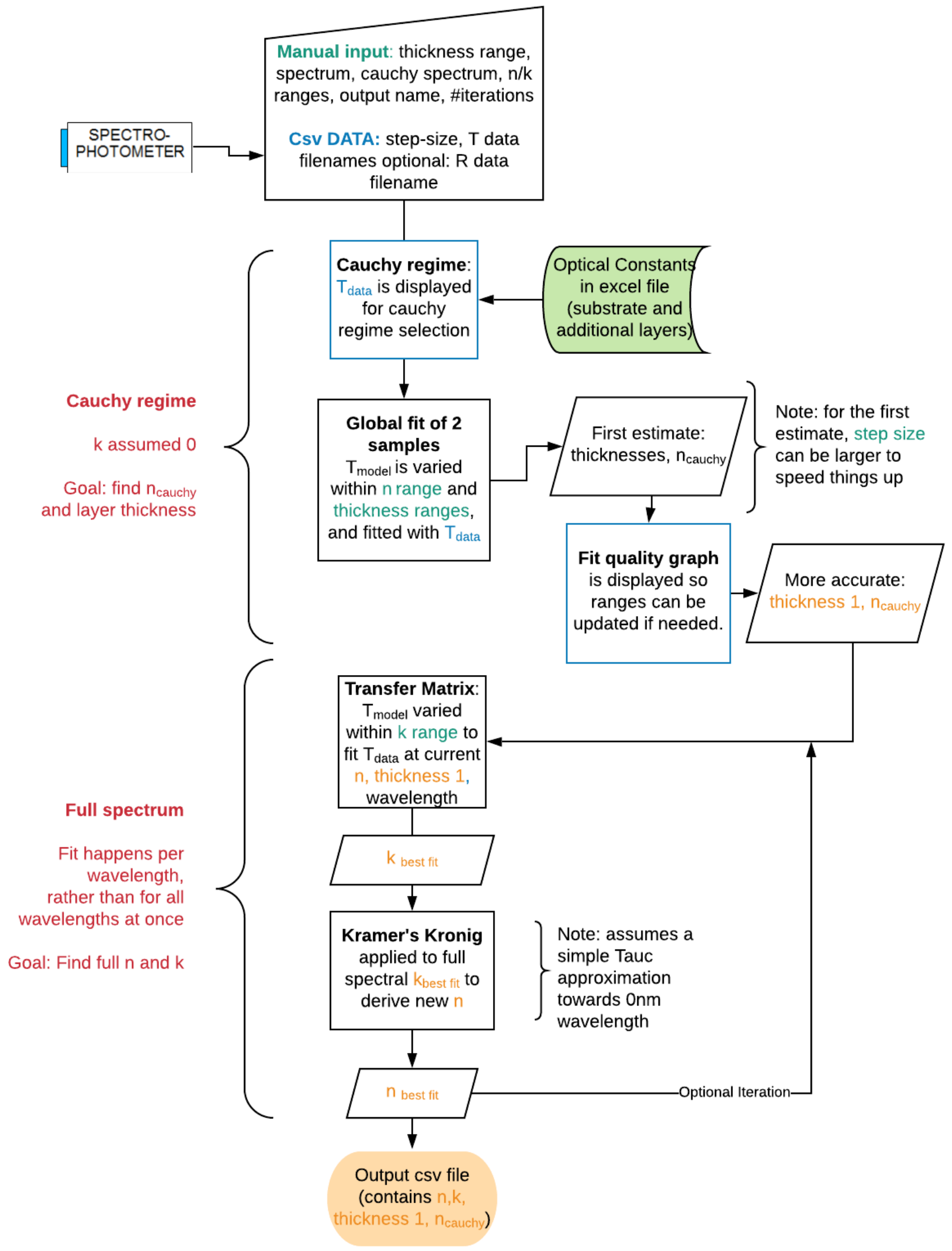

Flowchart 3C: Matab transmittance method script (NKFinder.m) 
The transmittance method script uses a fitting procedure as well, but rather than taking polarized reflectance as input (as in the case of ellipsometry), spectral transmittance data is fitted directly to modelled transmittance with the transfer matrix method. The free variables which are fit during the script are the thicknesses of the two samples, and the optical constants at each wavelength, $\eta(\lambda)$ and $k(\lambda)$.

Initially the same procedure as the ellipsometry method is followed: a Cauchy Regime is identified (easier to spot from transmittance data, since transmittance directly shows a drop where absorption begins), and is fitted to a Cauchy model equation, shown in Eq. 3.31 (with accuracy up to $B$ ). Two samples of different thickness (but identical Cauchy refractive index) are used to arrive at a satisfactory fit for the thicknesses as well, although in principle only one sample could be used while using a different method to obtain its thickness (such as profilometry or Cauchy regime ellipsometry).

Once an $\eta_{\text {Cauchy }}\left(\lambda_{\text {cauchy }}\right)$ is obtained, this value is assumed for $\eta$ at all absorbing wavelengths, and another transmittance fit is applied to either one of the transmission data sets in the absorbing regime, this time allowing $k(\lambda)$ to roam freely. Surprisingly, a very good fit will already be found for $k(\lambda)$, because in the absorbing regime $\eta(\lambda)$ tends to have a minor impact on the transmittance of the sample. A Kramers Kronig procedure is then applied to the $k(\lambda)$ data, using $\eta_{\text {Cauchy }}\left(\lambda_{\text {Mid-Cauchy }}\right)$ as the "known" constant in the solution of the Kramers Kronig differential equation (see Flowchart $3 \mathrm{~A}$ ). Another fit of $k(\lambda)$ can then be ran with the new, more accurate $\eta(\lambda)$ data, and this can be repeated until the data converges. In other words there are three main stages to the process:

- Cauchy regime fit where $k(\lambda)$ is assumed 0 and $\eta(\lambda)$ follows a Cauchy model fit to the data

- Full spectral fit where $\eta(\lambda)$ is fixed and $k(\lambda)$ is fitted to transmittance data

- Kramers Kronig is applied to $k(\lambda)$ to derive $\eta(\lambda)$,

where the final two steps are repeated if necessary, until $k(\lambda)$ and $\eta(\lambda)$ converge. 


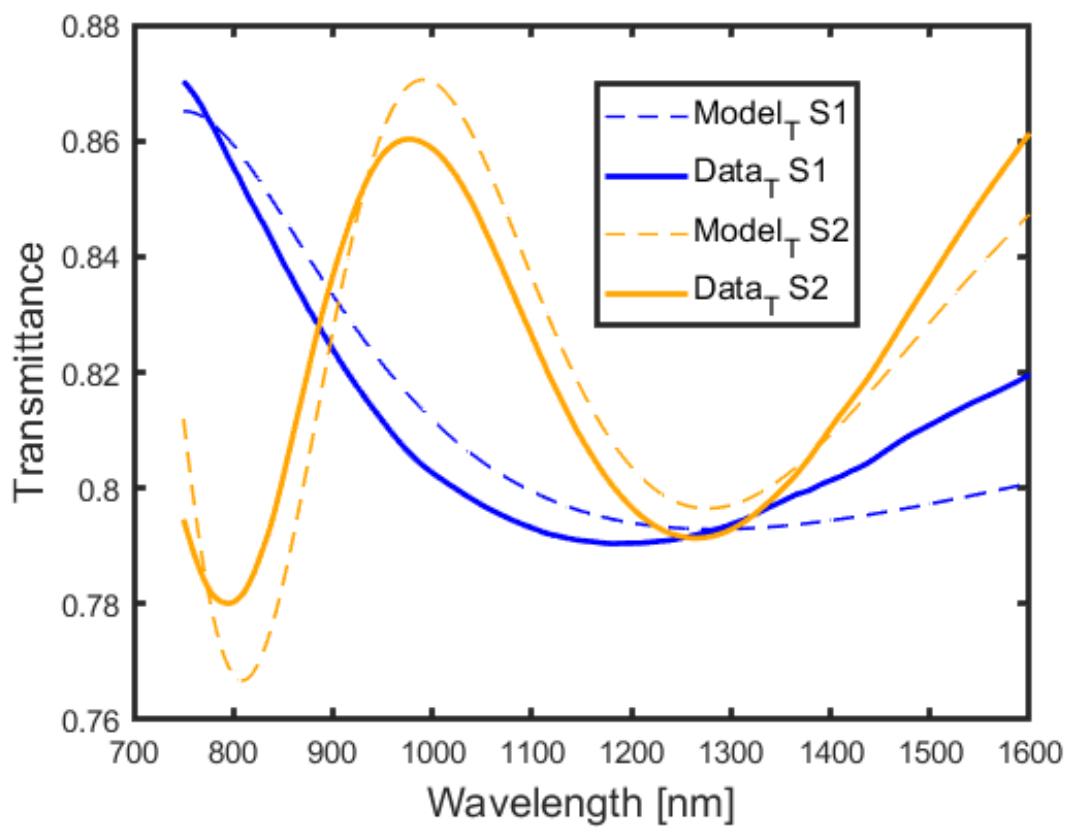

Figure 3.6. NKFiner Fit Graph

Figure 3.6: Example of the fit quality obtained from the Cauchy regime fitting procedure in a direct analogy with the first stage of fitting in ellipsometry.

A limiting factor on this method is the used "known" constant in the Kramers Kronig procedure, which follows from how good the thickness fit in the Cauchy Regime was. This is never adjusted in the repeating convergence and can therefore introduce a persisting error, which will be shown in the result by a discontinuous step between the Cauchy regime and the absorbing regime. Nevertheless, as long as the thickness fit is good, this step will be negligible and the data reliable and useful in transfer matrix models. The script allows for the quality of the thickness fit to be verified by showing both the real data and the Cauchy fit model data, as shown in Figure 3.6. 


\subsection{Application of the transmittance method}

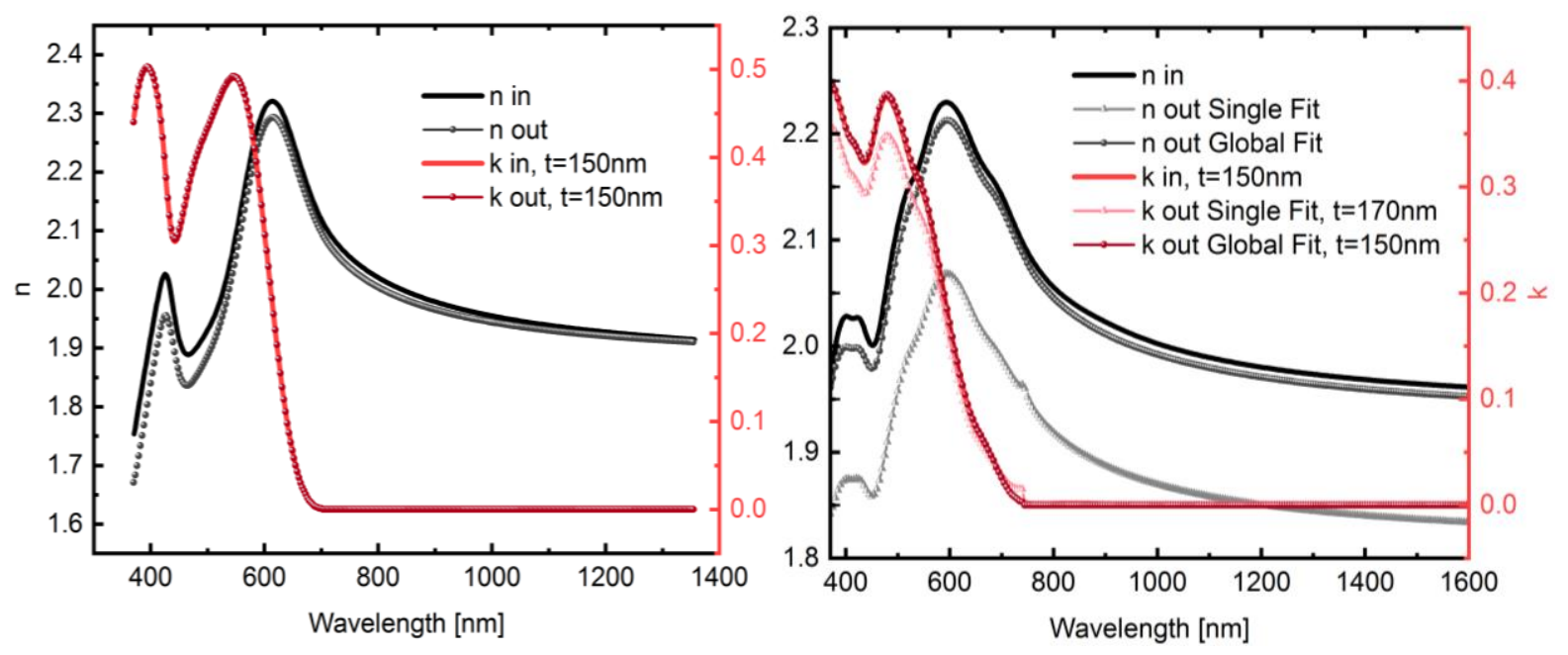

Figure 3.7. Initial NKFinder Test

Figure 3.7 Initial validation of the NK_Finder code using simulated transmittance data from input optical constants and re-deriving these optical constants. The input optical constants were derived from ellipsometry and verified in literature. Notice the increase in accuracy when using a global thickness fit to determine $n_{\text {cauchy. }}$ Left: PCDTBT, right: PC71BM.

The transmittance method was initially verified in steps, first the Kramers Kronig method was tested against known optical constant data of PCDTBT and PCBM, both common organic semiconductors in the literature ${ }^{68}$. The result of this is shown in Fig. 3.7. The transfer matrix script was also rigorously tested against spectrophotometer transmittance data and EQE data (which can also be derived with transfer matrix, as discussed previously). This was done for various, well-known organic materials, both neat and blends. It should be noted at this point that blended materials are not a problem for the methodology: the optical constants of the blend can be treated as one set of "effective" optical constants, tied to observables like absorptance, reflectance and transmittance, all of which have been previously linked directly to a Maxwell plane wave (or sum of plane waves) representation of the Electric field in the material.

After these basic modules were shown to function satisfactorily, the full transmittance method script was tested, first against modelled transmittance data of known optical constants (a sort of reverse function test), then against actual spectrophotometer 
transmittance data of PCDTBT and PCBM. The results of this data were then compared to the optical constants from literature and found to be accurate.

Finally, the script was put into practice by fabricating samples of non-fullerenes (both blend and neat layers) and perovskites on glass. This transmittance data was processed and compared to ellipsometry data, the results of which are shown in Figure 3.8 (neats), Figure 3.9 (blends) and Figure 3.10 (additional). 

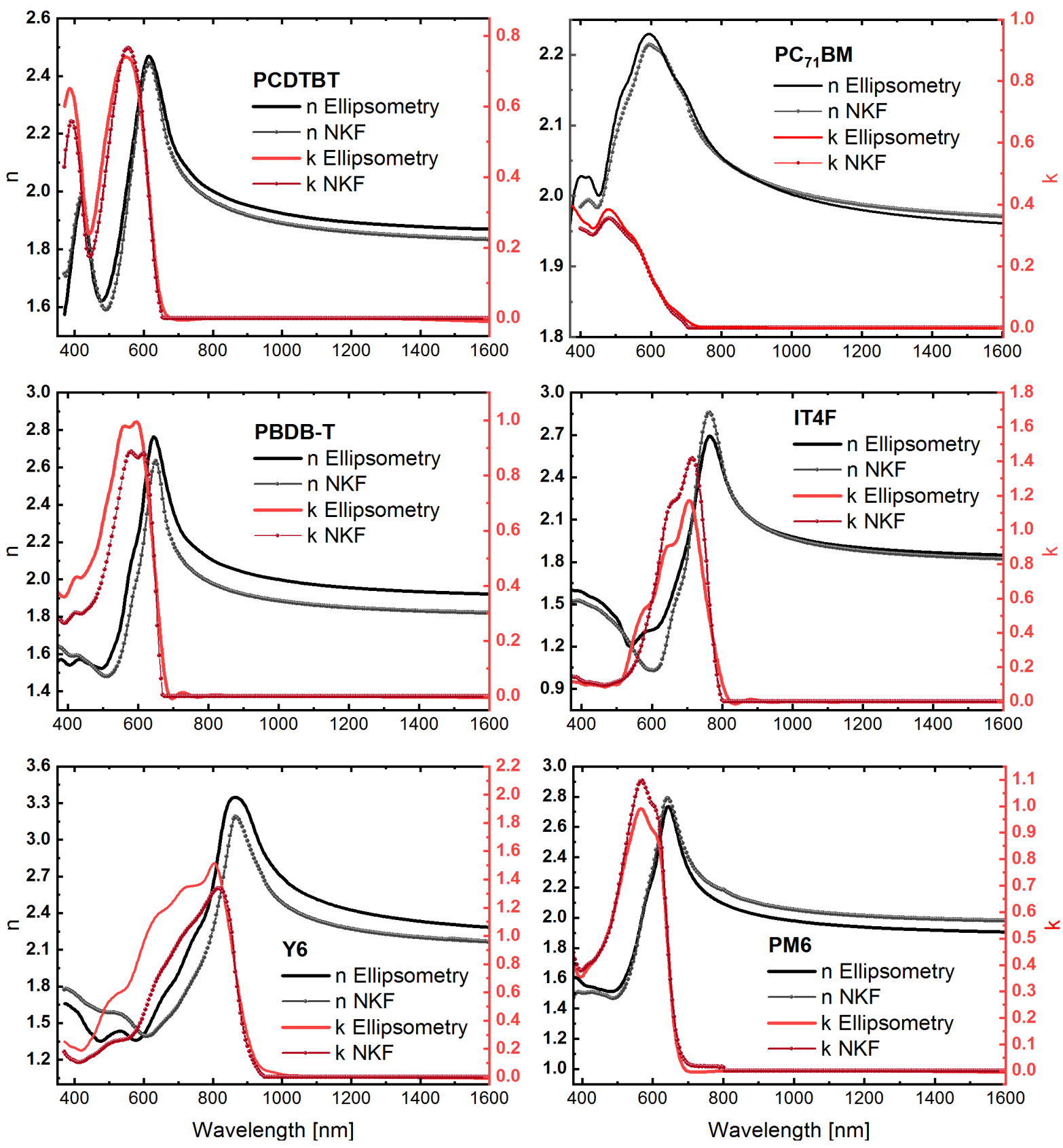

Figure 3.8 Neat Materials Results

Figure 3.8 Comparison of $\mathrm{n}$ - and $\mathrm{p}$-type organic semiconductors (neat materials) optical constants derived by ellipsometry (solid lines) and via the transmittance method (dotted lines, NKF stands for NK-Finder, the name of the matlab script). The refractive index ( $n$ ) and attenuation coefficients $(k)$ are represented on the left and right $y$-axis respectively. Note the non-fullerene acceptors Y6 and IT4F have significantly larger optical constants than their fullerene counterpart PCBM. 

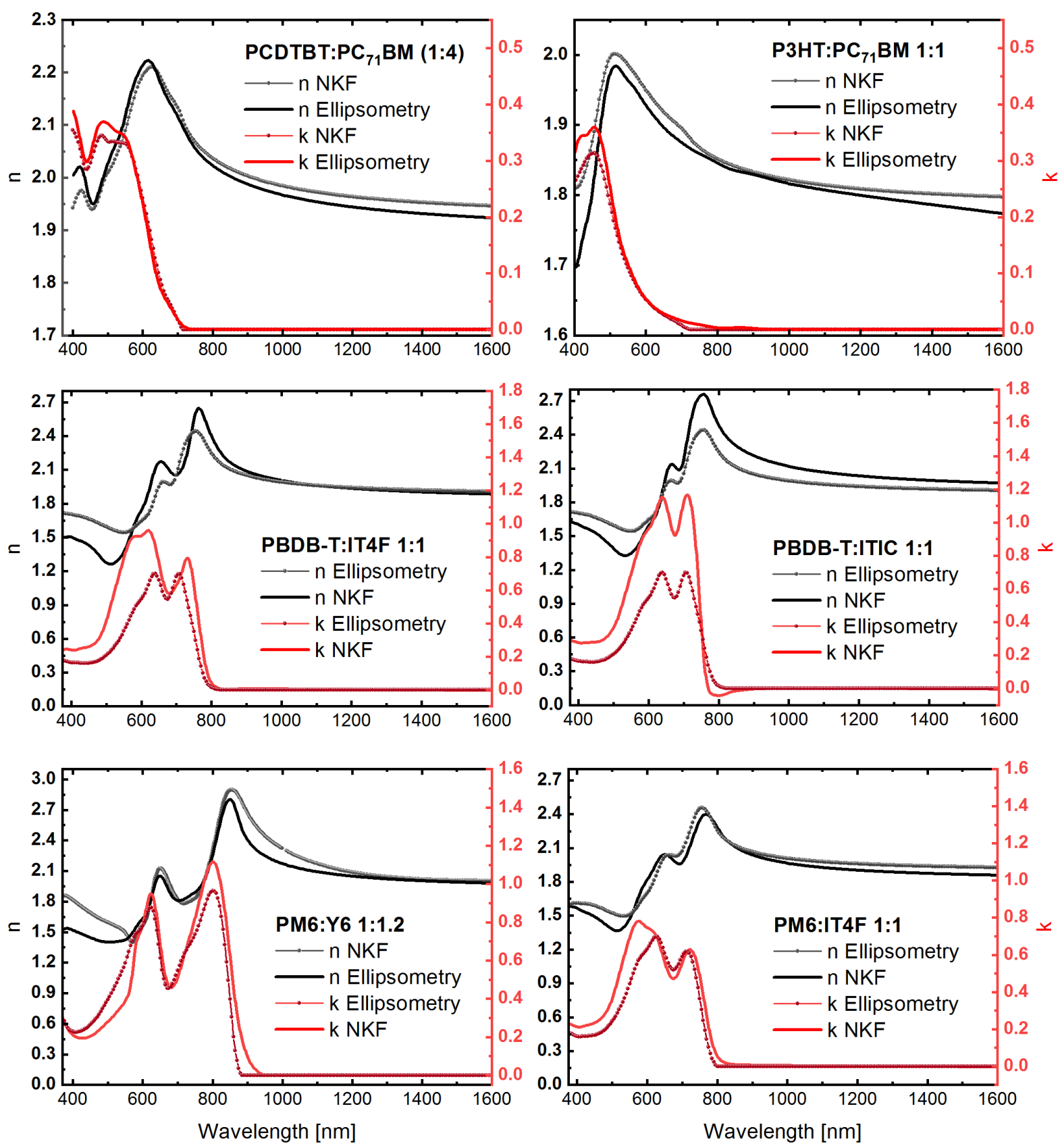

Figure 3.9. Blend Materials Results

Figure 3.9 Comparison of $n$-and-p-type organic semiconductors (acceptor-donor blends) optical constants derived by ellipsometry (solid lines) and via the transmittance method (dotted lines). The refractive index ( $n$ ) and attenuation coefficients ( $k$ ) are represented on the left and right $y$-axis, respectively. Note the high optical constants seen in the neat NFAs are translated through to the blends. 

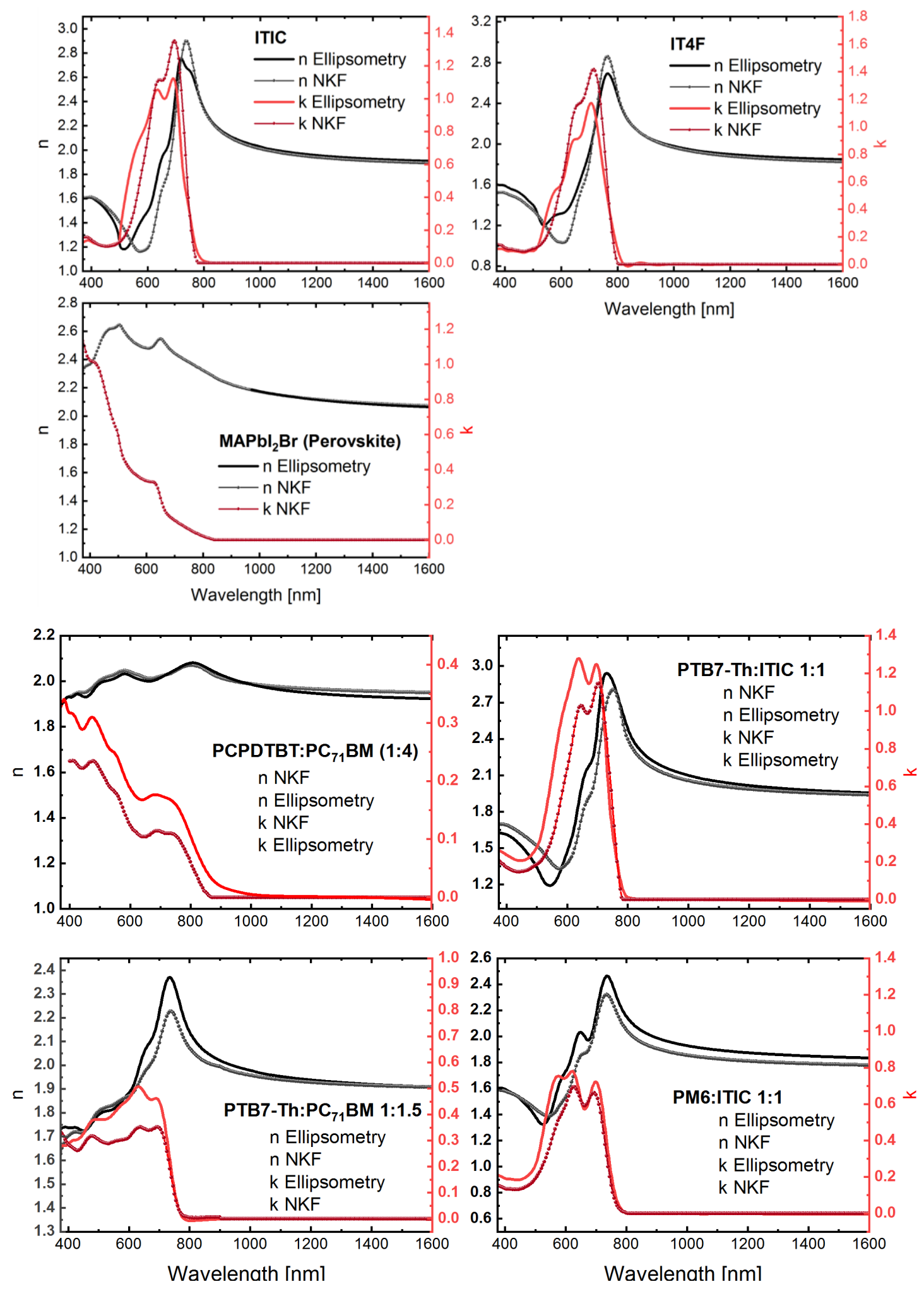

Figure 3.10 Additional Materials Results

Figure 3.10 Optical Constants for additional neats and blends that were not featured in the

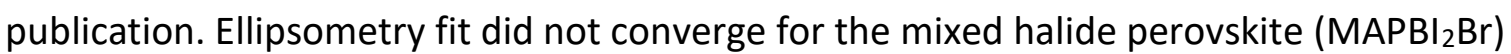




\subsection{Conclusion}

In this Chapter a detailed treatment of the theory behind optical constants and the relevant propagation of light through single and composite media using Maxwell's equations was provided. The technique of ellipsometry was likewise described - the most common means to determine the optical constants of materials in thin film form, including the use of effective media approximations. A new method relying on transmittance only measurements and the Kramers-Kronig equations was advanced and its implementation using Matlab described. The new method was applied to a number of perovskite and organic semiconductor (NFA) systems which, due to their relatively large optical constants are difficult to treat with ellipsometry. The new method proved suitable for these materials and delivered accurate optical constants for electro-optical analysis such as transfer matrix and EQE modelling and prediction. In this regard the method is a valuable new tool, as will be shown in the next Chapter which describes the analysis and understanding of a particularly complex next generation material solar cell architecture - the so-called carbon stack. 


\section{Application to third-generation devices in photovoltaics}

Once optical constants of single layers are known, these properties can be extrapolated to multi-layer stacks using the transfer matrix method. This way, complex photovoltaic devices can be described and modelled, to gain new insights into their functionality and to possibly optimize them to gain higher efficiencies. This chapter describes an in-depth analysis of several full devices.

This chapter is substantially based on works published by the author in Solar Rapid Research Letters ${ }^{69}$ and Advanced Functional Materials ${ }^{70}$

\subsection{Characterization of PCDTBT:PCBM Solar Cell}

4.1.1 Experimental tools and fabrication

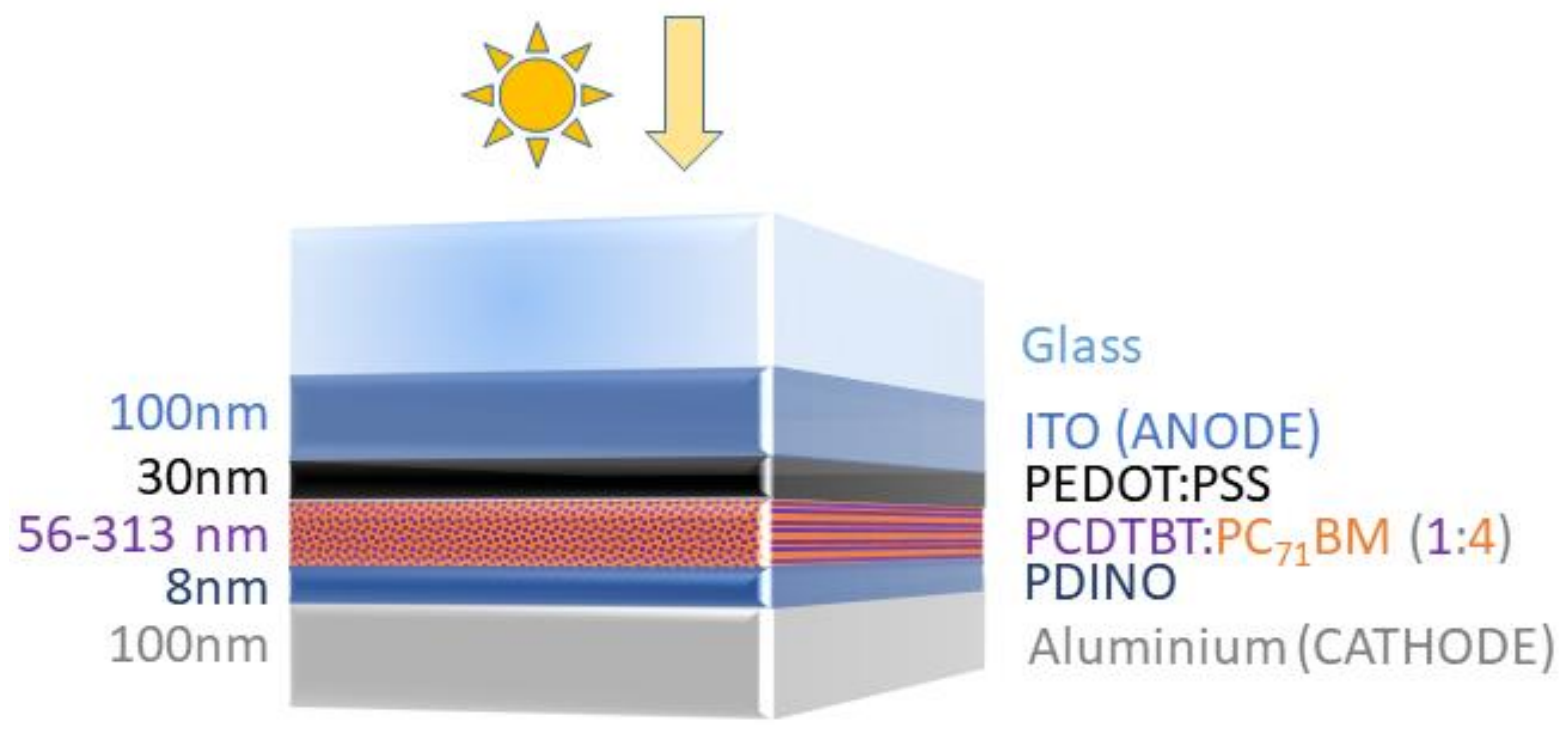

Figure 4.1. PCDTBT:PCBM Cell

Figure 4.1. A visual representation of a classic PCDTBT:PCBM cell, which was used to demonstrate the efficacy of the transmittance method script and transfer matrix method. Published in Advanced Optical Materials ${ }^{58}$ 
'The first solar cell system to which the NKFinder modelling tool was applied was the archetypal bulk-heterojunction system PCDTBT:PCBM. The basic structure of the device is shown in Figure 4.1 and has the following elements (from the illumination side):

1. An indium tin oxide coated glass substrate;

2. A thin $(30 \mathrm{~nm})$ PEDOT:PSS hole transport layer

3. A PCDTBT:PCBM BHJ active layer with blend ratio $1: 4$ by weight and of thickness varying between 55 and $315 \mathrm{~nm}$ );

4. A PDINO electron transport layer of around $8 \mathrm{~nm}$ and finally a reflective Al cathode.

This is a classic, second-generation organic solar cell with an active layer of a PCDTBT:PCBM blend, a so-called bulk-heterojunction layer (BHJ) as described in Chapter 1. PCDTBT is the electron donor and PCBM is the acceptor. This blend was chosen because it is already wellstudied in literature and relatively easy to fabricate. The electron transport layer is a material called PDINO, chosen for its high electron conductivity and relative environmental friendliness ${ }^{71}$. The hole transport layer is PEDOT:PSS, another common material in organic solar cells ${ }^{72}$, and the glass layer has an imprinted layer of transparent indium tin oxide (ITO), which is patterned into cell active surfaces and channels that lead to the edge of the substrate where silver can be painted on for contacts, according to the scheme in Figure 4.2.

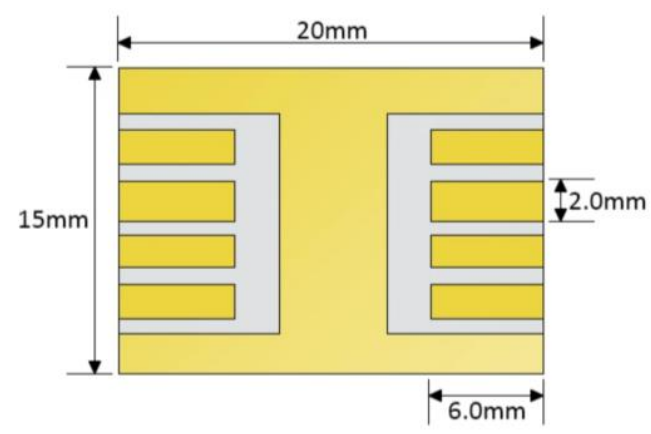

Figure 4.2. ITO Substrate Schematic

Figure 4.2. Schematic of the ITO print on the solar cell glass substrate, modified upon reproduction from Ossila ${ }^{73}$

The commercially patterned ITO glass substrates (Ossila) are cleaned in an Alconox solution bath at $60{ }^{\circ} \mathrm{C}$, followed by a sonication in sequence in Deionized (DI) water, acetone and 2propanol for 10 minutes each. After treatment with UV-Ozone (Ossila, L2002A2-UK) for 
10mins, $30 \mathrm{~nm}$ of PEDOT:PSS is deposited uniformly on the substrate using a spin-coating technique. Next, the solution for the 1:4 PCPDTBT:PC71BM is achieved as follows: PCPDTBT and PC71BM (1:4) are dissolved in a Chlorobenzene:Dichlorobenzene (CB:DCB) (1:1) mixture at $70^{\circ} \mathrm{C}$, at a concentration of $40 \mathrm{mg} \mathrm{ml}-1$. The PCDTBT:PCBM films are then also spin-coated on top of the PEDOT:PSS, at varying RPM (rounds per minute) speeds to vary the thickness. Afterwards, $10 \mathrm{~nm}$ of PDINO is cast on the active layer from a methanol solution (1 mg $\mathrm{ml}-1$ ). Finally, $100 \mathrm{~nm}$ of $\mathrm{Al}$ is evaporated on the PDINO to form the cathode.

For the evaporation we employ tungsten boats which are heated in a vacuum to a temperature at which the aluminium starts to evaporate. The cells are placed in a mask which ensures the aluminium is deposited in a pattern which isolates the 8 different cells (see Figure 4B) from each other. After this the cells can be encapsulated with glue and glass to ensure stability, but these particular cells are stable enough to be measured without degrading on the short term.

Cells with a varying active layer thickness ranging from $56 \mathrm{~nm}$ to $313 \mathrm{~nm}$ were fabricated (thickness range $=[56,90,113,149,197,313])$. The same spin speeds were used to deposit the blend on glass, and these corresponding thickness values were verified with both ellipsometry and transmittance method measurements. Optical constants $\eta(\lambda)$ and $k(\lambda)$ were also derived for all materials using the methodologies outlined in chapter 3 and found to be near identical for both ellipsometry and the transmission method (see Figure 3.8). NKFinder values were subsequently used in the EQE modelling process.

The cells were then characterized as described in Chapter 2, by acquiring both the AM1.5G JV characteristics and external quantum efficiency (EQE) between $350 \mathrm{~nm}$ and $900 \mathrm{~nm}$. The results of this are provided in Figure 4.3 a) and b). 

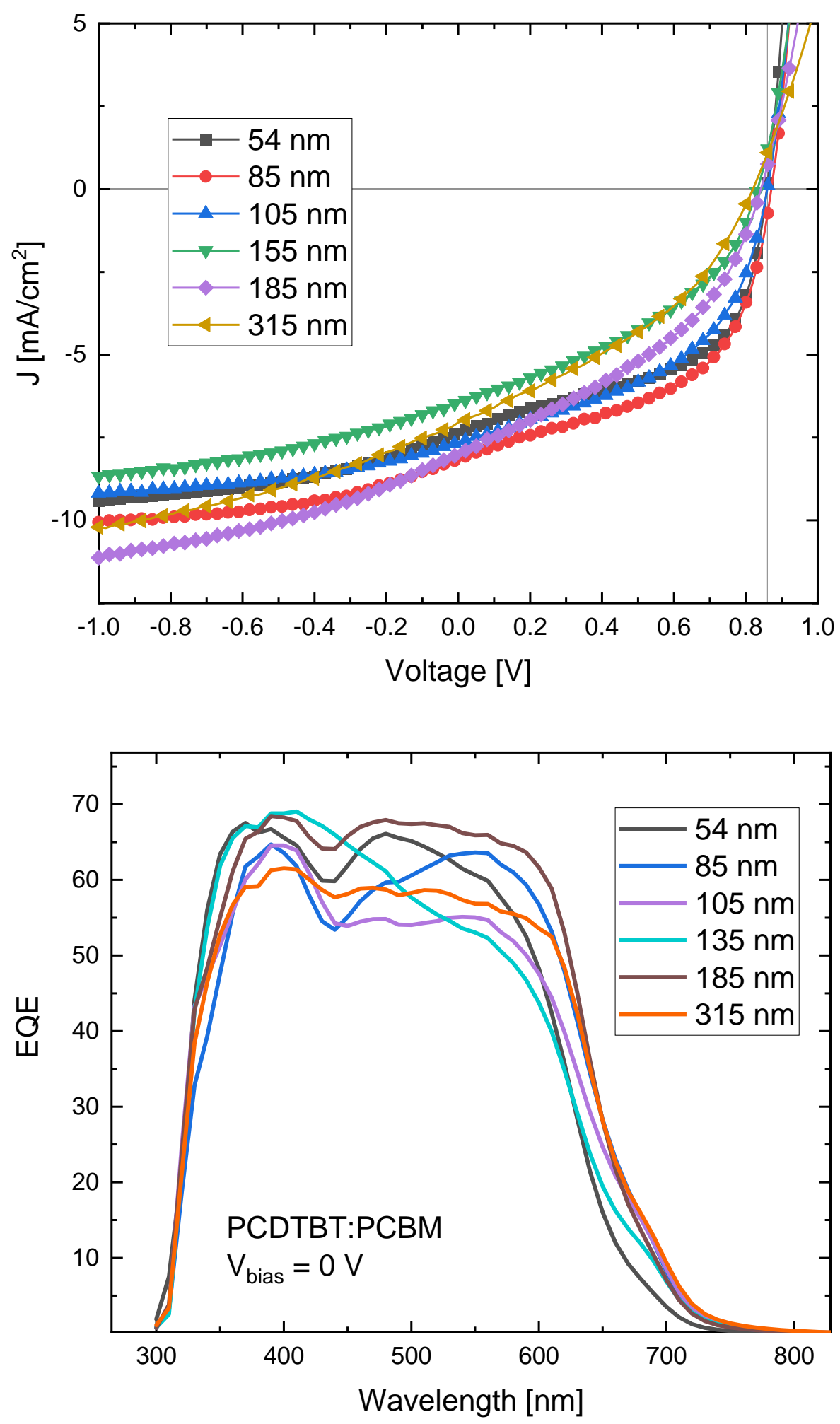

Figure 4.3 JV And EQE of PCDTBT:PCBM Cell

Figure 4.3 a. $J V$ measurements of several PCDTBT:PCBM solar cell devices with varying active layer thickness. B. EQE measurements of the corresponding pixels on the cells. Thickness of the active layers of the cells is shown in the legend. 


\subsubsection{Modelling of the internal quantum efficiency (IQE)}

As can be seen in Figure 4.3, the EQE of the devices can vary quite significantly with active layer thickness. Recall from Chapter 1 that External Quantum Efficiency is given as:

$$
\operatorname{EQE}(\lambda)=\frac{\operatorname{IQE}(\lambda)}{1-R(\lambda)-T(\lambda)-A_{p}(\lambda)}
$$

and that the reason for this spectrally varying efficiency has two important components:

- How much light is absorbed by the active layer (characterized by the denominator)

- How much of the generated charge is electrically transported to the electrodes (characterized by the IQE and the numerator).

The measured EQE is affected by both these components. If we can therefore determine how much light is absorbed by the active layer, we can also extract the IQE from these measurements. While $R$ and $T$ can be measured, the parasitic absorptance poses a problem, and must be calculated with the transfer matrix method described in Chapter 2. For the sake of clarity, a flowchart is shown detailing the specific implementation of a transfer matrix script coded in Matlab in provided in Flowchart 4A. 


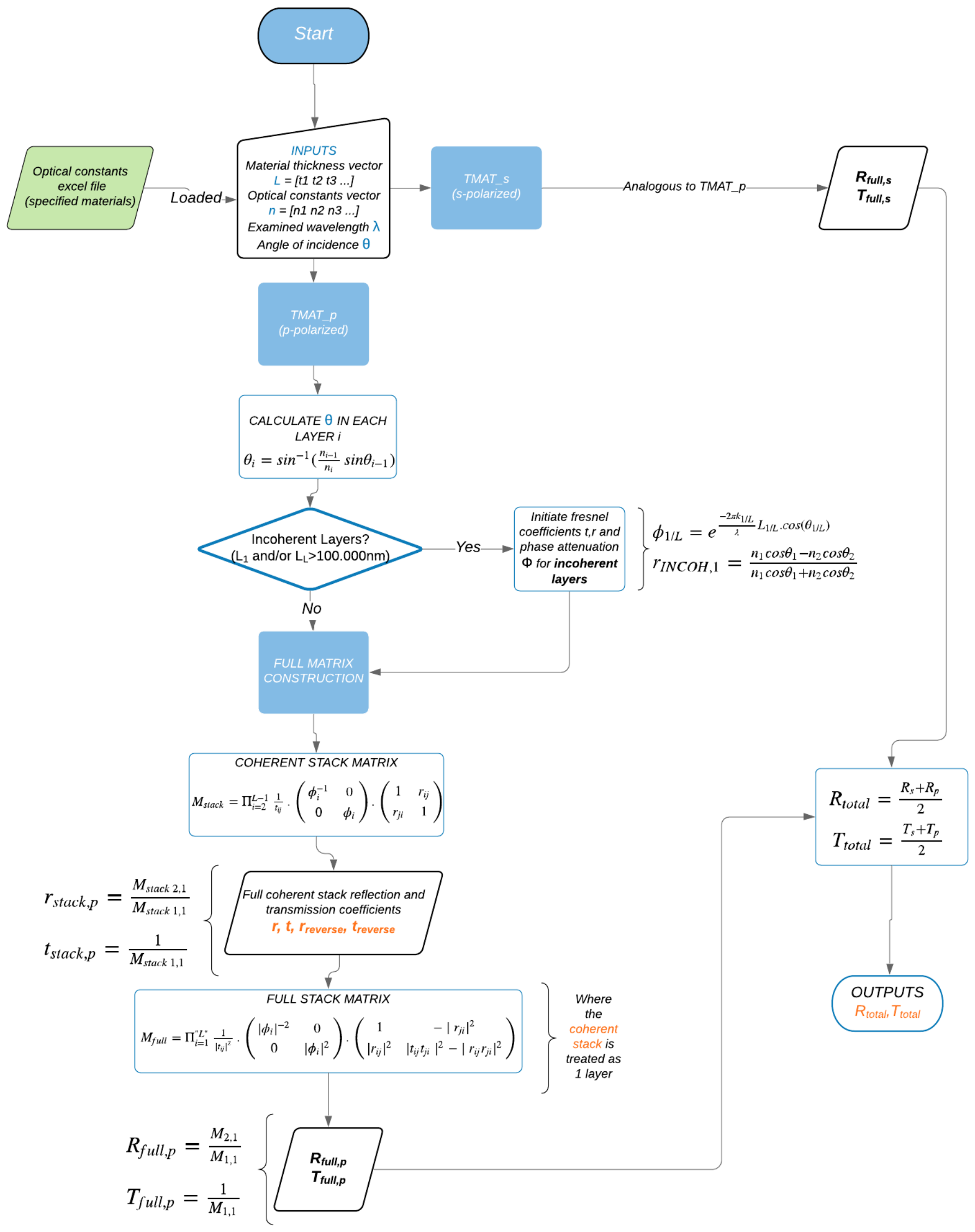


Flowchart 4A (previous page): An overview of the transfer matrix implementation in matlab. This method also incorporates the possibility of different angles and incoherent layers, and is based on the work of Harbecke ${ }^{47}$ and Ohta et al. for the deduction of internal electric fields (not shown here) ${ }^{74}$.

The respective layer thicknesses and optical constants must be input in the script to predict the Reflection, Transmission and most importantly the parasitic and active contributions of the Absorptance. Once these are known the IQE can be calculated.

The absorptance of the active layer can be deduced directly from the transfer matrix, once calculated. Recall that the matrix determines the electric field amplitude at every layer boundary (see Figure 4.4). This field can be extrapolated across the layer as a simple sum of the two plane waves (forwards- and backwards-travelling), by definition. Once the total electric field is known, this can be used to calculate and integrate active layer absorptance $A_{A L}$ across the layer, using

$$
I_{0}(\lambda) A_{\mathrm{AL}}(x, \lambda)=\frac{1}{2} c \varepsilon_{0} \alpha_{\mathrm{AL}}(\lambda) \eta_{\mathrm{AL}}(\lambda)\left|E_{\mathrm{AL}}(x, \lambda)^{2}\right|,
$$

Where incident intensity $I_{0}$ can be taken as the AM1.5 intensity and $\alpha_{A L}$ can be derived from the active layer imaginary optical constant $k_{\mathrm{AL}}$, using Eq. 3.19:

$$
\alpha_{\mathrm{AL}}(\lambda)=\frac{4 \pi k_{\mathrm{AL}}(\lambda)}{\lambda}
$$
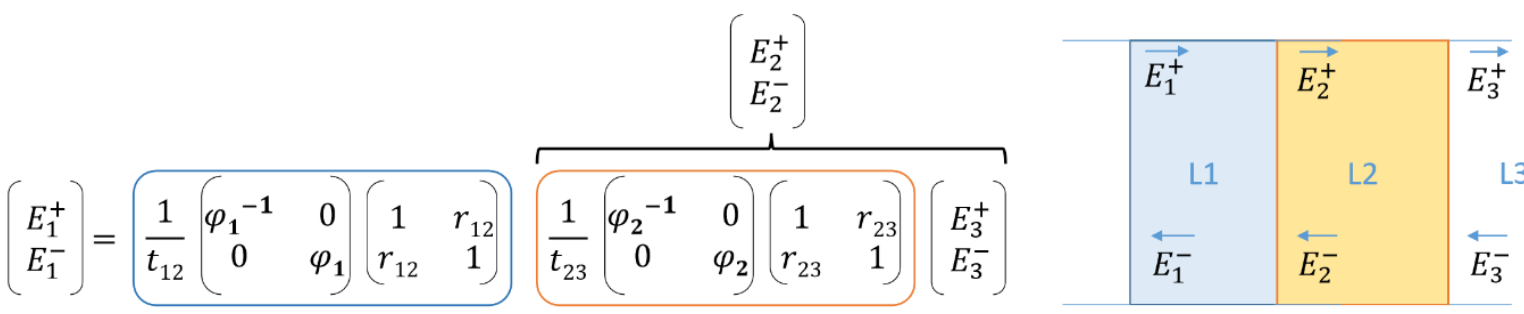

Since with the assumption in Eq. 4.1,

$$
\mathrm{EQE}_{\text {ideal }}=A_{\text {active layer }}=\int_{0}^{t_{A L}} \int_{\lambda_{\min }}^{\lambda_{\max }} A_{\mathrm{AL}}(x, \lambda) d x d \lambda,
$$


this is the simulated absorptance or EQE under ideal internal collection. The ratio of the modelled, ideal EQE to the experimental EQE is shown in Figure 4.4.

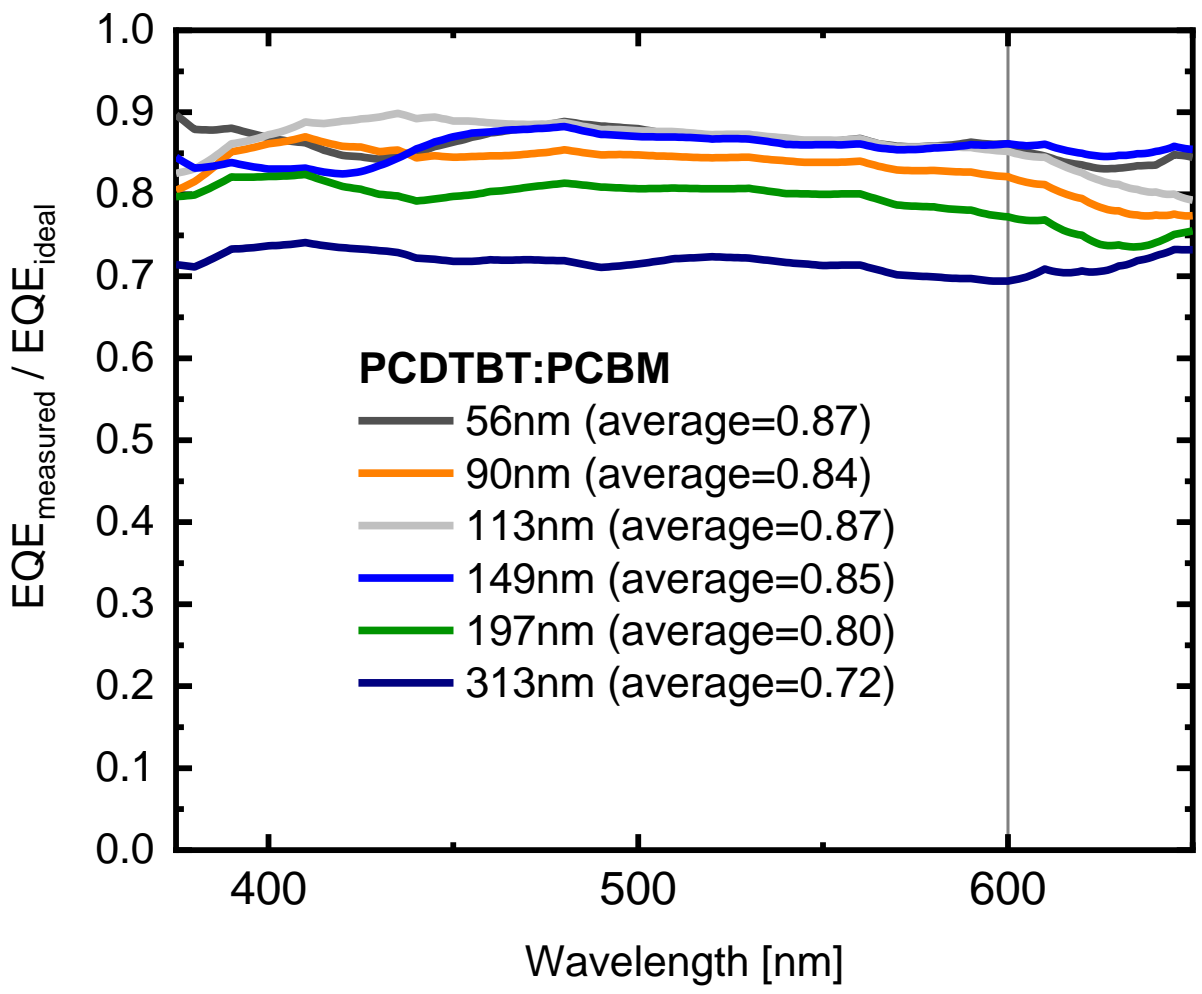

Figure 4.4. PCDTBT:PCBM EQE Ratios

Figure 4.4 Ratio of the experimentally measured EQEs to the modelled, ideal EQE, showing spectral uniformity in charge collection for every device.

As can be seen, their ratio is spectrally more or less constant, except at the edges of the bandgap where charge generation is small and noise in the measured EQE will therefore be too large to have a meaningful comparison. This spectrally quasi-constant ratio implies that the IQE is in fact spectrally constant, a finding which is in line with the assumption that highenergy excitons are collected with the same efficiency as low-energy excitons (see Chapter 2). If we therefore take the average value of these spectrally quasi-constant ratios, we can assume it to be equal to the IQE for each active layer thickness, again following Eq. 2.15 and 4.1:

$$
\mathrm{IQE}=\frac{\mathrm{EQE}_{\text {measured }}}{\mathrm{EQE}_{\text {ideal }}}=\frac{\mathrm{EQE}_{\text {measured }}}{A_{\text {Active Layer }}}
$$


A comprehensive comparison demonstrating the validity of our assumptions can be seen in Figure 4.5, which compares model to experiment. As can be seen, a trend in IQE with thickness also exists: IQE starts reducing significantly with active layer thicknesses above $200 \mathrm{~nm}$. This seems natural given our earlier discussion of charge transport (Chapter 2): the thicker the layer the higher the bimolecular recombination coefficient.
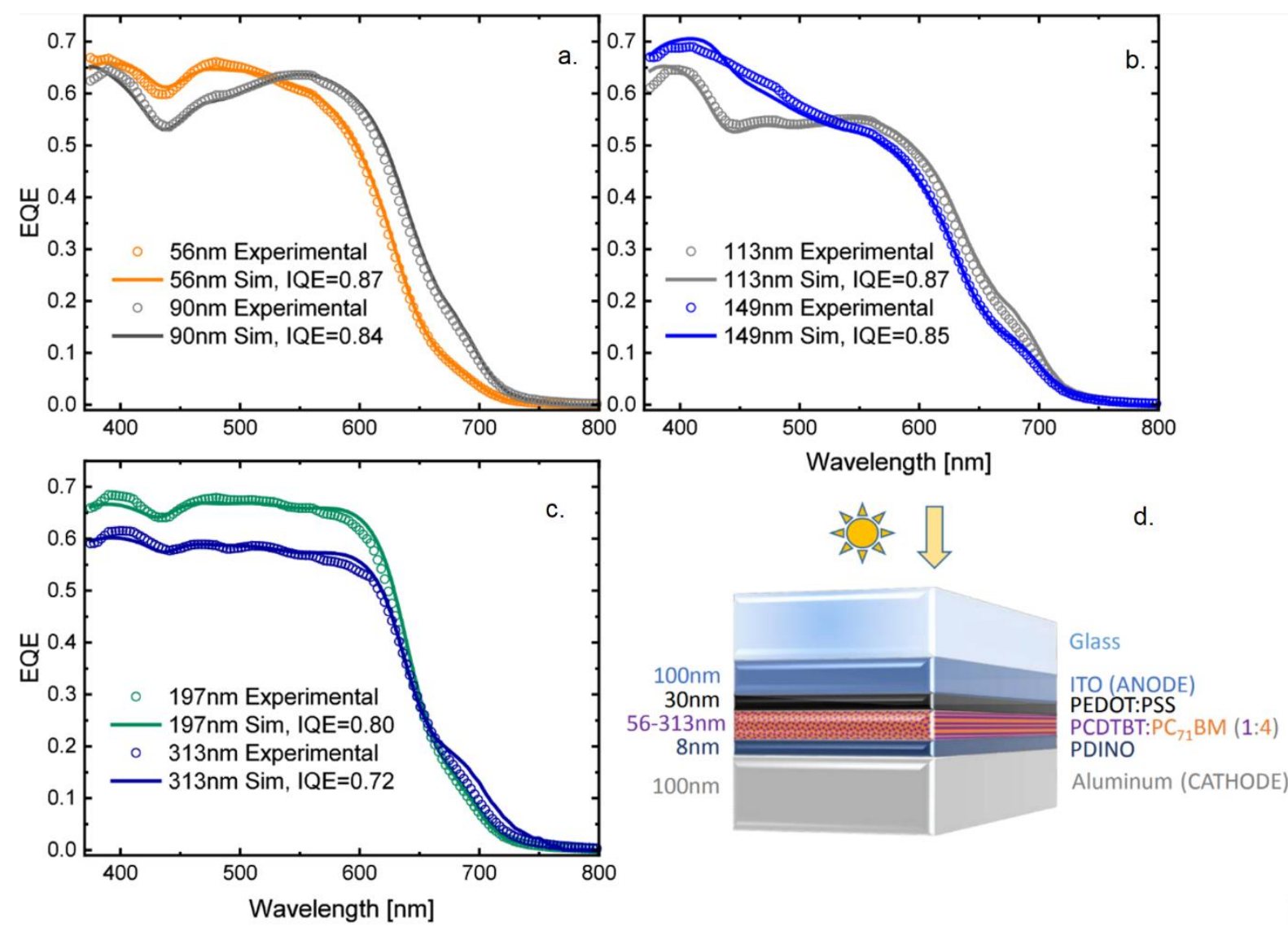

Figure 4.5. IQE Fitting For PCDTBT:PCBM Cell

Figure 4.5. Spectrally constant IQE fitting of the modelled EQE with the experimentally measured EQE values of the thickness-varying solar cells. ${ }^{58}$ a-c. Comparisons of the modelled and measured EQE curves for active layers varying from $56 \mathrm{~nm}$ thickness to $313 \mathrm{~nm}$. d. visualisation of the PCDTBT:PCBM solar cell stack.

Now that a robust overview of modelling a standard organic cell has been demonstrated, the same procedure can be applied to more complex, third generation solar cells. 


\subsection{Perovskite Mesoporous Carbon-contact Solar Cell}

\subsubsection{Introduction}

The Perovskite Mesoporous Carbon-contact Solar Cell is a novel solar cell architecture recently developed ${ }^{75}$ with the intention of facilitating industrial-scale fabrication. This cell design contains thick mesoporous zirconia and titania layers on the order of a micron, topped off with a mesoporous carbon electrode (anode), and infused with a perovskite semiconductor. The carbon anode is composed of nano-particulate graphene crystals conferring electrical conductance ${ }^{76}$. In principle, all layers of this stack are printable, and indeed, various groups are now producing mini-modules with power conversion efficiencies of $>6 \%$ (aperture area of $198 \mathrm{~cm}^{2}$ ) using amino valeric acid-methyl ammonium lead iodide (AVA-MAPI3) as the perovskite semiconductor; see, for example, the work of Watson and coworkers $^{29,77-79}$. The zirconia, titania, and carbon layers of the modules of Watson and coworkers were screen printed from porous pastes and the MAPI3 "infused" into the stack post deposition and annealing. The schematic of Figure 4.6 shows a typical carbon stack structure, a notable feature being the relatively thick carbon (around $10 \mu \mathrm{m})$, zirconia $(800 \mathrm{~nm})$, and titania $(1200 \mathrm{~nm}$ ) layers. These form a considerably thicker "junction" than other perovskite architectures. The SPECIFIC Innovation and Knowledge Centre provided the example devices which were examined for modelling and analysis in this work. 


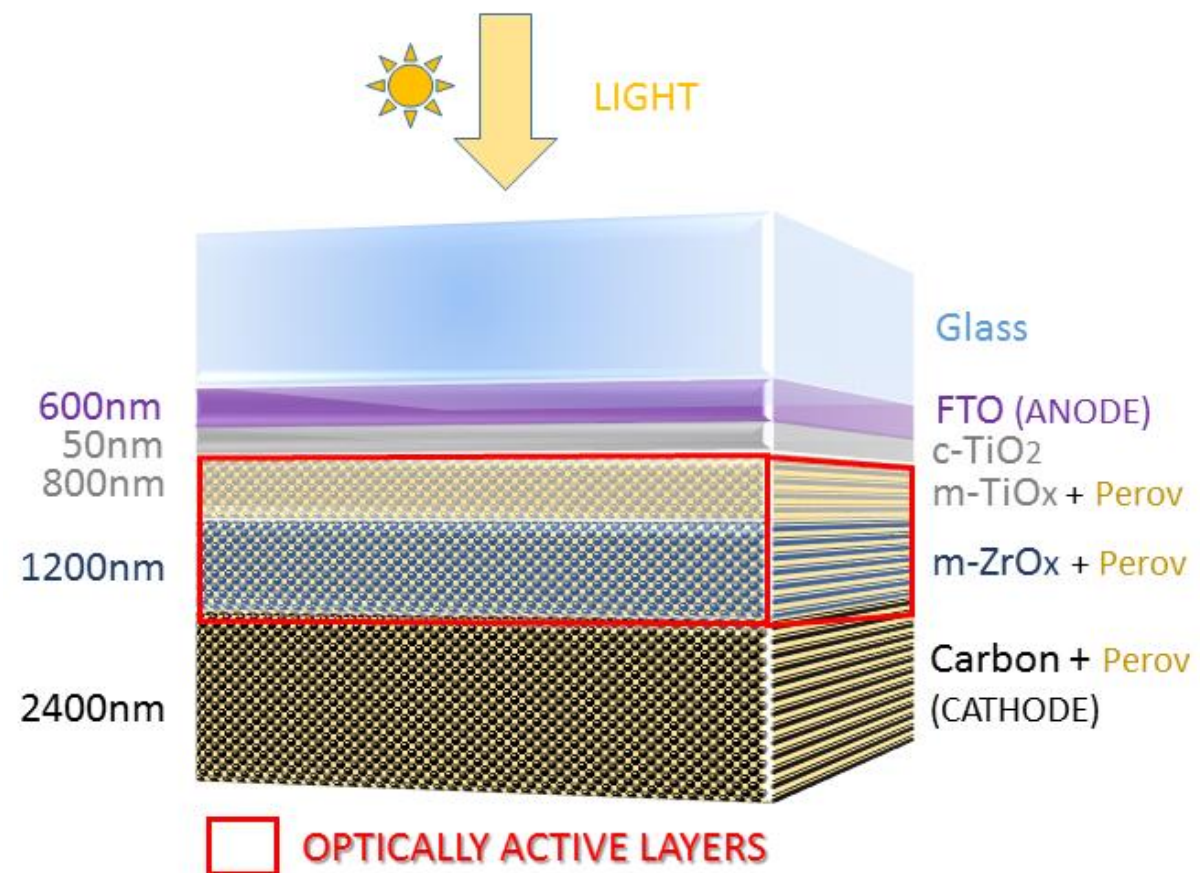

Figure 4.6. Mesoporous Perovskite Carbon Solar Cell

Figure 4.6. Visual representation of the mesoporous carbon solar cell architecture. Note that instead of an ITO on glass layer, this cell uses a Fluoride Tin Oxide (FTO) layer, which is better matched to the titania energetic band levels.

It is the AVA-MAPI perovskite semiconductor which is the actively absorbing material in the cell, although certain other parts of the cell might in fact absorb and contribute to collected charge as well (this will later be shown by the simulation process). For most organic solar cells the relatively thick layers would severely impact collection efficiency, however the organohalide perovskites have charge carrier diffusion lengths in the micrometre range ${ }^{80}$ and an exciton dissociation that is expected to be near instantaneous at room temperature (exciton-binding energy on the order $2 \mathrm{meV}^{81}$ ), meaning the majority of charge transfer is likely electron/hole transport instead ${ }^{81}$. The thickness of the active layer is desirable for the reason that thin layers will vary significantly in relative thickness error during mass production, which can cause a relatively high variance in cell performance (in fact, this is one of the reasons why Silicon solar cells are so well-suited for industrial production, the high mobility of silicon allows for thick junction solar cells, up to $\mathrm{cm}$ scale depending on the crystallinity of the silicon) $)^{82}$. 
In short, there are several aspects that are special about this solar cell architecture and which need to be investigated for characterization according to the previously described procedure.

\subsubsection{Determination of energetic structure}

Unlike in the previously discussed PCDTBT:PCBM cell, where the charge collection is already well-studied ${ }^{83-85}$, it first becomes necessary to determine how charge carrier generation and collection takes place in the cell. This is important not only because it might explain certain things about the collection process, but also because we need to know which layers are actively absorbing and contributing to the collected charge, in other words it needs to be made clear which material's light absorptance is purely parasitic.

While the electronic energy structure of the other materials is known from literature ${ }^{75}$, the graphene crystals' energetic structure could not be readily verified, and therefore had to be measured. A Kelvin probe was used for this. The Kelvin probe setup works by depositing (screen-printing) a thin layer of the graphene on glass, attaching a metal reference clamp to the graphene, and bringing a gold-tipped probe close to the material. When electrical connection is made between the probe and the sample, electron flow can occur in the direction of the lower to the higher Fermi level. This electron flow causes the equilibration of the probe and sample Fermi levels. A surface charge then builds up on the probe and the sample, with a related potential difference known as the contact potential $\left(V_{c}\right)$. After some calibration, an external potential, known as the backing potential $\left(V_{b}\right)$ can be applied to null the charge between the probe and the sample. When the charge is nulled the Fermi level of the sample returns to its original position. This means that $V_{b}$ is equal to $-V_{c}$, which is the work function difference between the probe and the sample (it is thus necessary to accurately know the work function of the gold probe as well). Therefore, the work function of the examined material can be determined from this backing voltage. 


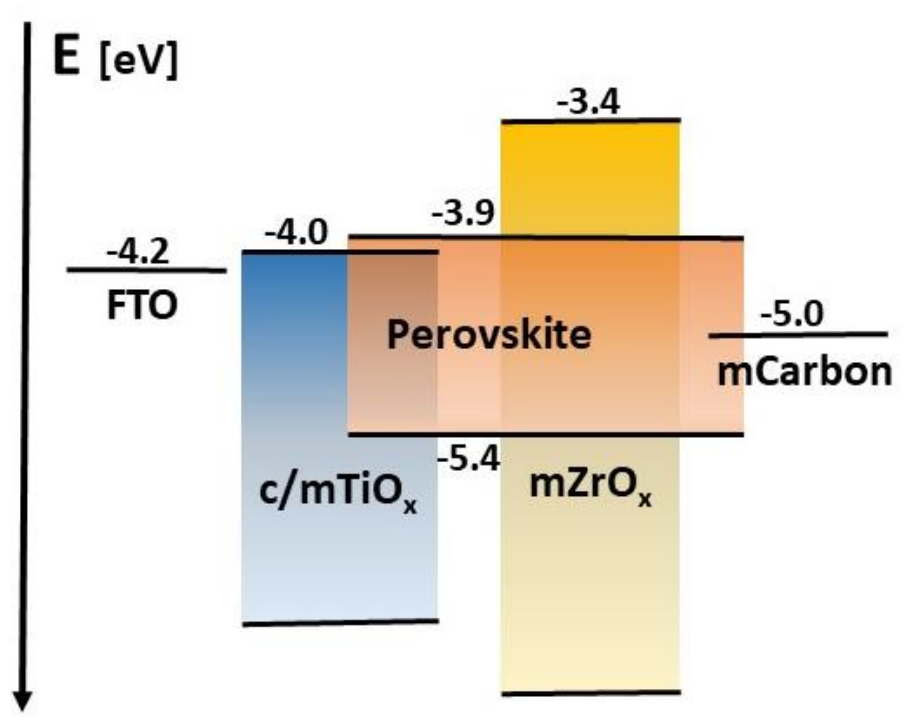

Figure 4.7. Carbon Cell HOMO/LUMO

Figure 4.7. Energy band and HOMO/LUMO diagram of the involved materials. The perovskite energy levels are close to the $\mathrm{mTiO}_{x}$, but far removed from the $\mathrm{ZZrO}_{\mathrm{x}}$ conduction band, which makes the zirconia act purely as a spacer layer.

Shown in Figure 4.7 is a diagram of the resulting work function energy level of the carbon, alongside the energy structures of the other materials. The perovskite is throughout its whole layer in close contact with either the Titania and Zirconia, since it is infiltrated into these mesoporous layers. Note that it is also contacting a significant portion of the mesoporous carbon, since it is only infiltrated after all the other layers are deposited.

It is clear from this energy structure that, with no voltage applied, electrons generated in the perovskite will flow towards the lower energy levels through diffusion (see Eqs. 2.20), through the titania into the Fluoride Tin Oxide (FTO). At this point an important distinction due to the hybrid inorganic-organic structure of the perovskite arises. While for band structures in purely inorganic materials, a big difference in energy levels is not necessarily a problem, remember that organic (or hybrid organic) materials may use the statistical "hopping" mechanism, which decreases significantly in efficiency if the energy level difference between adjacent materials (and with it, Fermi overlap of their wave functions) increases ${ }^{86}$. This is the reason for the Titania layer in the first place, since its energy level is intermediate between the two adjacent materials and it will therefore increase efficiency of charge transfer between them ${ }^{86}$. Similarly, holes will diffuse towards higher energy levels, as electrons from those levels fill up the available spaces. However, the relevant Zirconia band level (the conduction band) is too 
far removed energetically to have any meaningful carrier flow. The mesoporous zirconia layer therefore only acts as a buffer layer between the titania and the graphene contact. This makes sense since otherwise electrons could flow from the perovskite or titania to the graphene contact, which ideally would only collect holes as a cathode.

However, one should note that it is still possible for both electrons and holes to flow to the carbon contact from the perovskite itself, some of which is also directly contacting the carbon. Since the graphene crystals energy level is located in between perovskite HOMO and LUMO, the mesoporous carbon contact is not a selective contact. This is important to keep in mind when moving on to the full opto-electrical model.

\subsubsection{Mesoporous layers}

The mesoporous nature of the carbon stack architecture creates additional challenges for understanding light coupling and propagation in the structure, and indeed, determining the appropriate optical constants of the constituent layers. This challenge can be addressed by implementing an effective medium approach, which is a mathematical method that approximates the refractive index of the layer as an averaged refractive index of the composite materials. There are several different effective medium approaches, but for this work the Bruggeman effective medium approach was used, which can be derived according to 87.

$$
f_{a} \frac{\varepsilon_{a}-\varepsilon}{\varepsilon_{a}-2 \varepsilon}+\left(1-f_{a}\right) \frac{\varepsilon_{b}-\varepsilon}{\varepsilon_{b}+2 \varepsilon}=0,
$$

Where $f_{a}$ and $\left(1-f_{a}\right)$ represent the probabilities of finding the respective materials of dielectric constants $\varepsilon_{a}$ and $\varepsilon_{b}$ in a spherical space and can be interpreted as a volume ratio. $\varepsilon$ is the unknown variable and the effective dielectric constant of the mesoporous layer, from which the effective optical constant can be deduced. Since this approximation still requires knowledge of the exact fractional volume occupation of the different composite materials (in this case, perovskite/air and Titania/Zirconia/Graphene), experimental methods had to be used to determine these exact ratios in each layer. Samples of mesoporous materials on glass were subjected to ellipsometric porosimetry with dynamic toluene infiltration, the results of which are shown in Figure 4.8. 


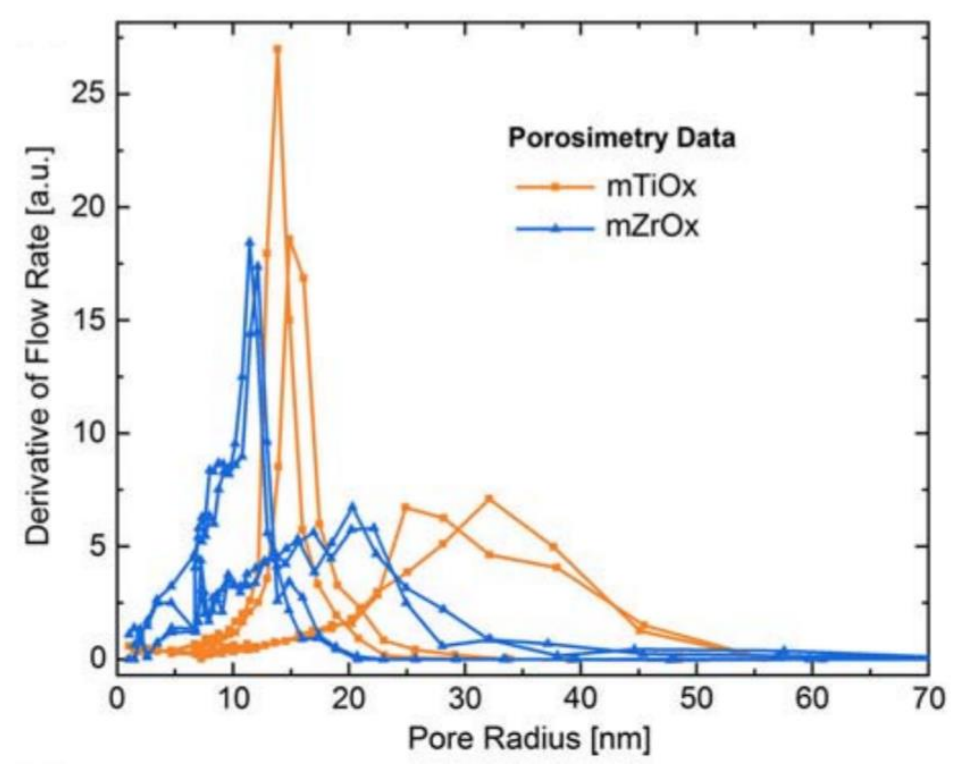

Figure 4.8. Porosimetry Data

Figure 4.8 Left: results of dynamic porosimetry measurements to determine volume fraction of air. High flow rate derivative means a good fit was found around this pore radius value.

The pore volume fraction was determined to be approximately 0.5 in both cases, which was then plugged in as a parameter in the ellipsometric calculation together with known data for perovskite optical constants ${ }^{22}$. It should be noted that a full perovskite infiltration was assumed in calculating the effective indices, a process facilitated by the AVA component in the perovskite. Although some studies have confirmed this assumption ${ }^{70}$, this still remains an approximation. The resulting optical constants for the active, perovskite-infiltrated layers are shown in Figure 4.9. 


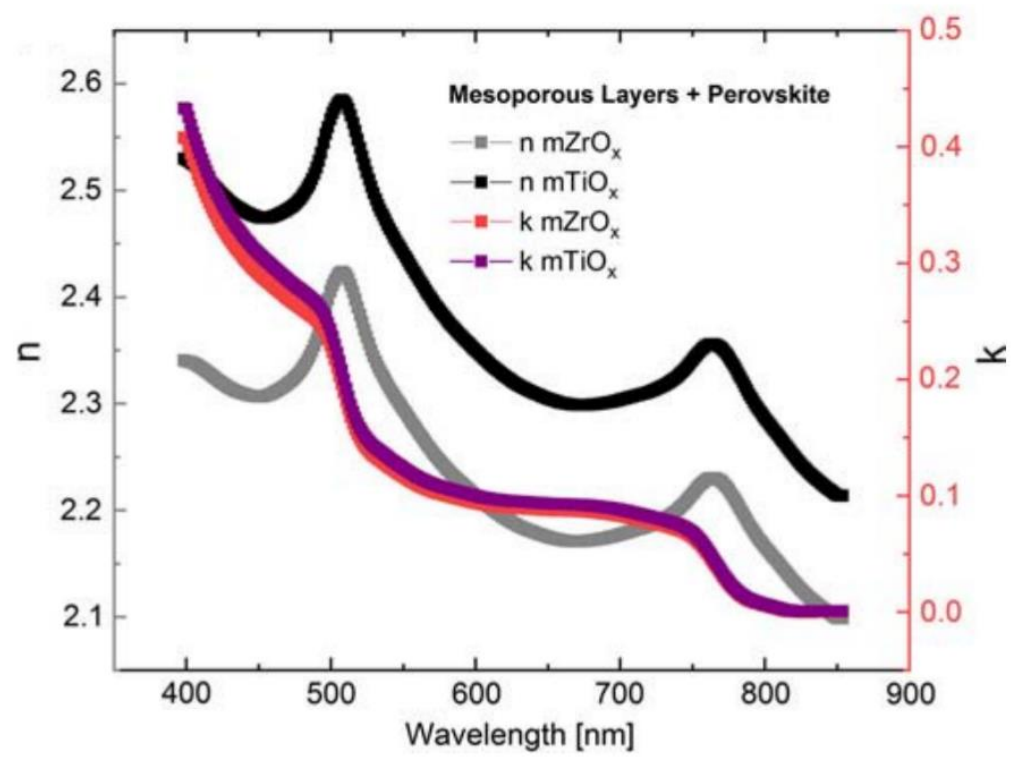

Figure 4.9. Optical Constants For Carbon Cell

Figure 4.9 Resulting optical constants of the active layers, derived using ellipsometry and the Bruggeman effective medium approximation.

This data could consequently be implemented in a transfer matrix model to derive the full optical generation profile of the stack, which could in turn be used to derive a complete optoelectrical model of the novel stack as outlined below.

\subsubsection{Full opto-electrical model}

Running the stack layer data in a transfer matrix model can yield several useful results. The first property that is usually determined (after reflectance and transmittance) is the internal electric field distribution at varying wavelengths of incident light. This can be extrapolated to a charge generation profile according to Eqs. 2.27 and 2.28, and the resulting profile for two key incident wavelengths are shown in Figure 4.10. 


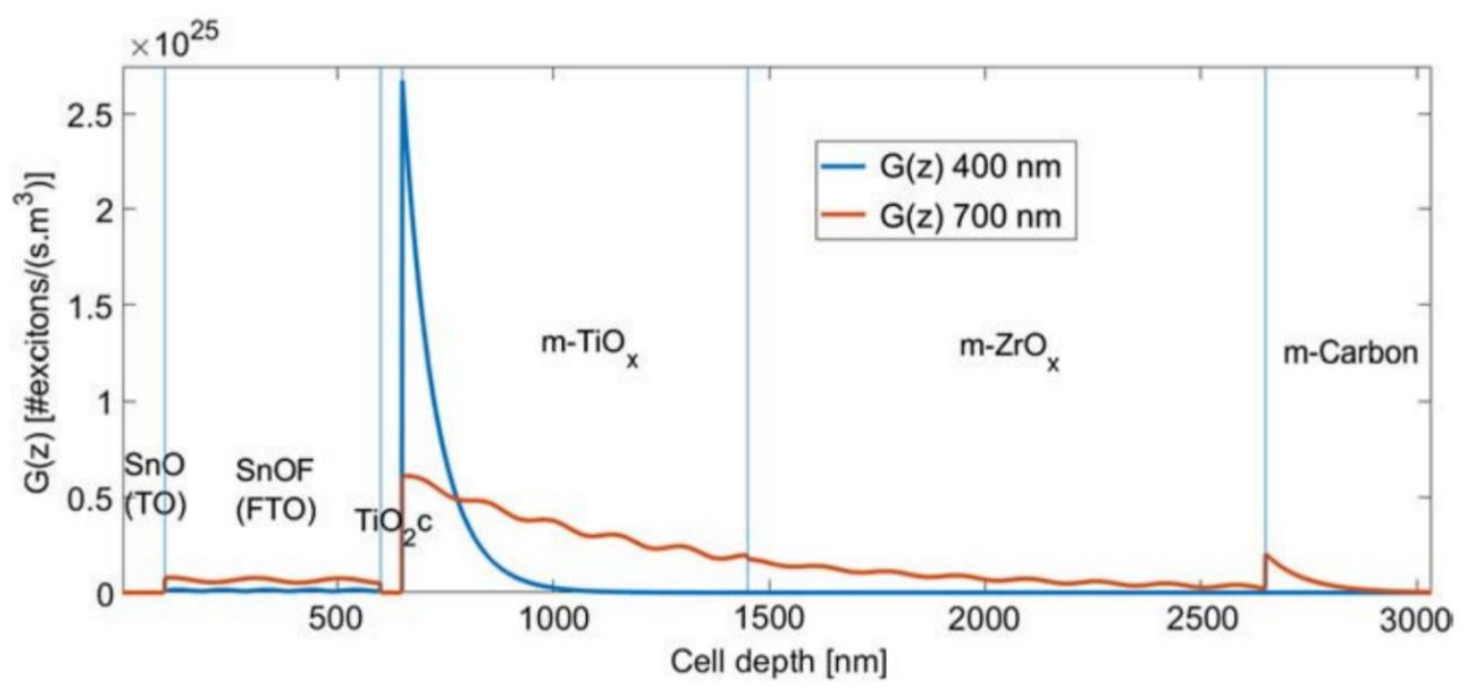

Figure 4.10 Generation Profile For Carbon Cell

Figure 4.10 Spatial profile of photo-generated charges in the multilayer carbon stack solar cell at 400 and $700 \mathrm{~nm}$ incident light wavelengths (as examples). The generation profile is determined from a transfer matrix simulation of the optical field distribution assuming unity internal quantum efficiency. Light is incident from the left and propagates to the right.

One of the stack properties that can immediately be observed is that most of the light at the lower wavelengths only generates charge in the mesoporous Titania layer, close to the anode of the device. The stack is unusually thick for an organic (or rather, hybrid organic) device but as discussed before this becomes possible due to perovskite's low exciton binding energy and high mobilities, in other words recombination will be low even though (in the case of $400 \mathrm{~nm}$ light) the holes will have to travel a relatively long way to the carbon contact. In fact it is a common property for perovskite opto-electronical devices that the major bottlenecks for charge collection efficiency depend on the interfacial recombination between the layer surfaces ${ }^{86}$. It can also be noted that towards the low energy end of the absorption spectrum $(700 \mathrm{~nm})$ the light penetrates into the carbon contact, meaning the carbon itself will also absorb some of the light. Since there is no mechanism within the carbon contact to effectively drive charge separation (it is a non-selective contact, as seen in Figure 4.7), it can be assumed that recombination for charge generated here will be much higher, which means the IQE for this layer will be significantly lower (in fact from subsequent model analysis it can be assumed to be virtually zero). 
By comparing the combined absorptance of the two active layers, the perovskite-infiltrated m-zirconia (mZrOx) and m-titania (mTiOx), with the actual measured EQE, we can make an estimate for the IQEs of the separate layers. Again, we will assume that the generated charges relax energetically and are collected with the same efficiency no matter what energy they are excited at in a single layer. In other words, collection efficiency is fitted to be spectrally constant, yet allowed to be different for both layers.
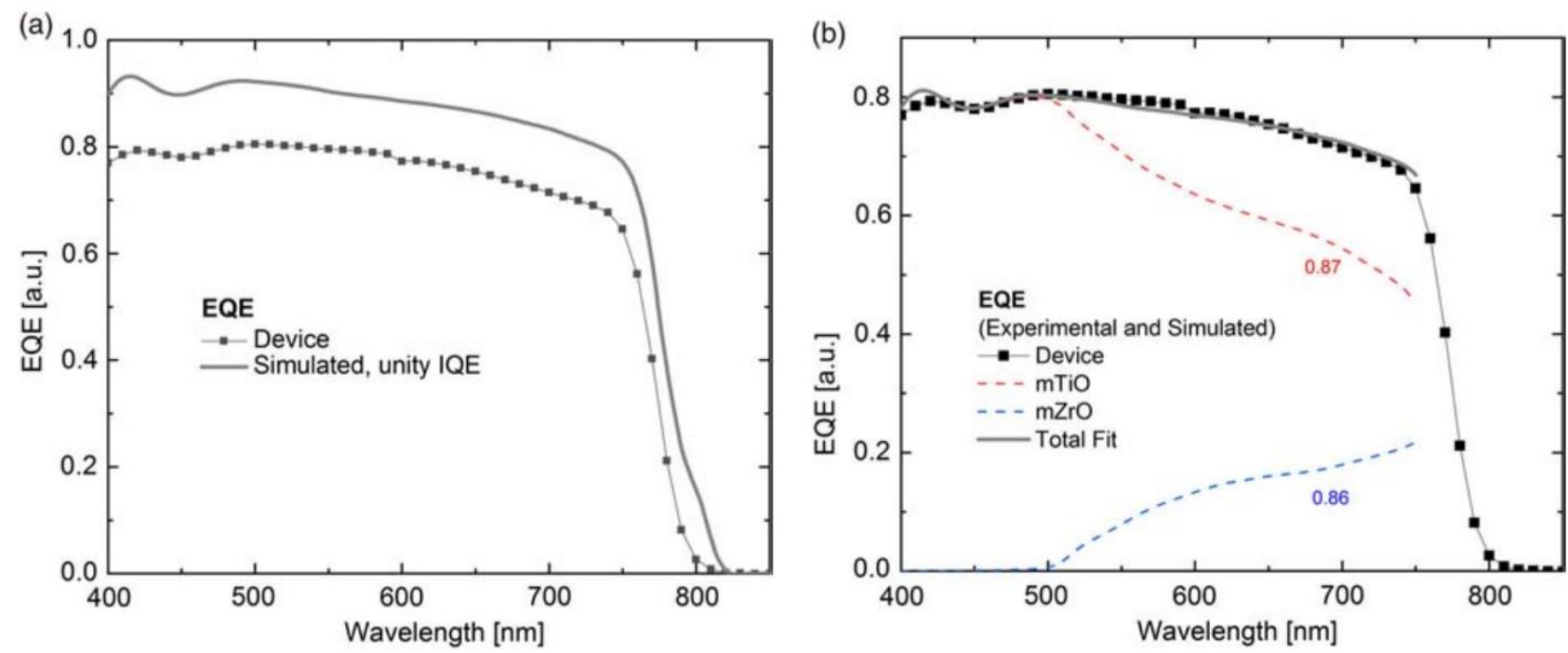

Figure 4.11. IQE Fitting For Carbon Cell

Figure 4.11 External quantum efficiencies (EQEs)-experimental values compared with simulations for the case of a) unity internal quantum efficiency (IQE) and b) non unity, spectrally flat IQEs determined from a two parameter fit (Figure S5, Supplementary Information). The analysis yields IQEs of 0.87 and 0.86 in the titania and zirconia layers, respectively.

The result of this fit is shown in Figure 4.11. The fit yields a somewhat surprising result. The efficiency is near equal for both layers. While for the mTiOx layer there is an energetic driving force for the electrons to transfer to the Titania (thus reducing the chance of recombination), in the Zirconia layer there is no such driving force (the Zirconia does not interact electrically with the perovskite, as mentioned previously). This can be partially explained by the fact that even at the most penetrating wavelengths most of the light is still absorbed close to the Titania, which acts as a sink for electrons and thus creates a concentration gradient that drives diffusion in the Zirconia as well. While this is a somewhat intuitive way to look at the process, further electrical investigation is warranted to confirm this hypothesis. 
Since the charge generation profile has been calculated, electrical simulations can now be performed. For this the drift-diffusion solver software Setfos was used. Setfos implements drift-diffusion equations (Eqs. 2.) across all layers using the generation profiles calculated with the transfer matrix (such as those shown in Figure 4.9) and solves the equations in terms of current, voltage and charge carrier concentration. Results are shown in Figure 4.12 and Figure 4.13 with accompanying information. In short it becomes clear from these results that the active layer thickness plays an essential role, across key metrics such as open circuit voltage and short-circuit current density, in subverting the losses from the non-selective contact. This is a qualitative assertion, as accurate information about the fill factor and other aspects of the stack electrical properties were only estimated as model inputs. However, the figure trends show a clear picture as to the role of active layer thickness in the stack. 

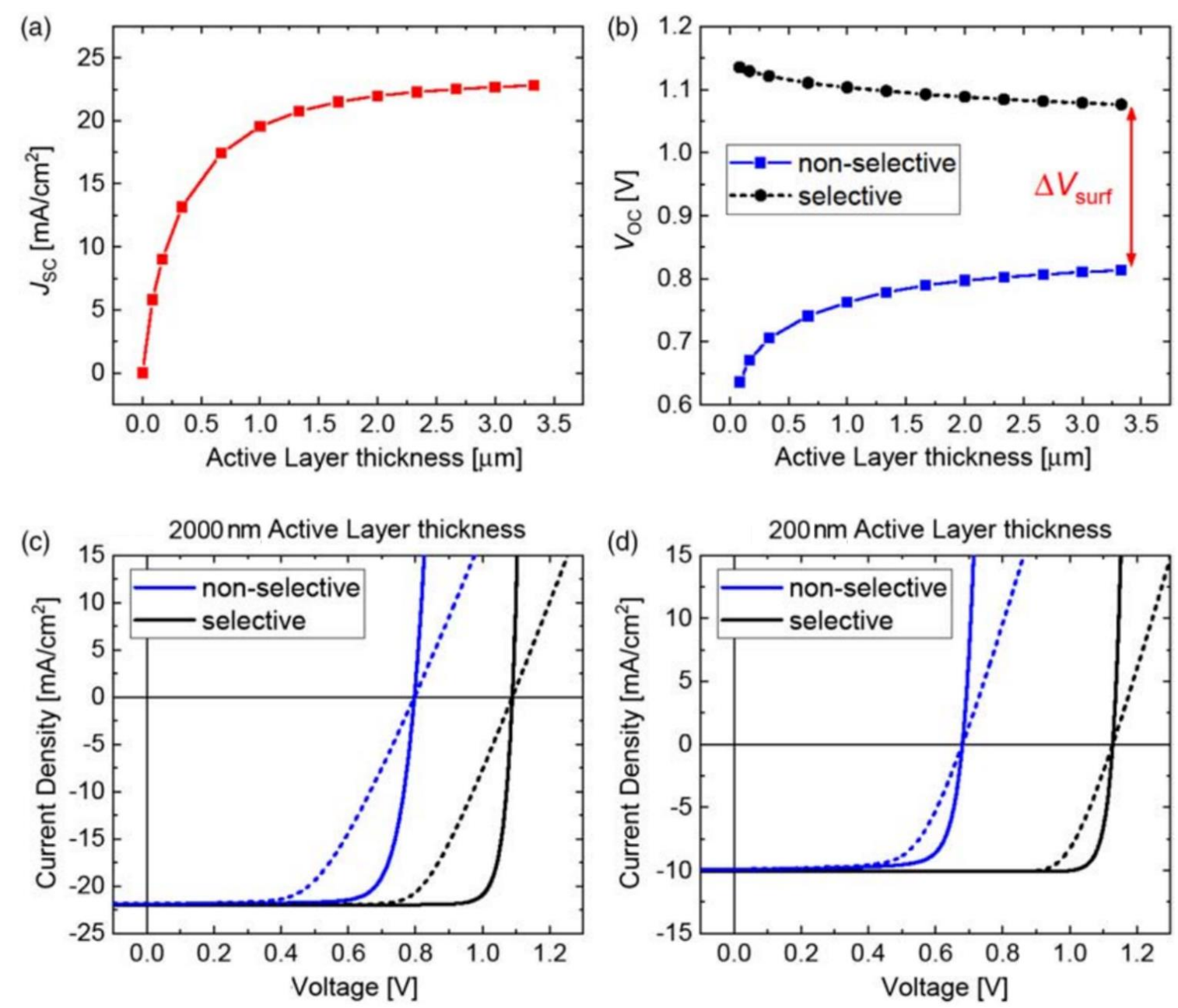

Figure 4.12. Simulated Thickness Dependence for Carbon Cell JV

Figure 4.12 Simulated thickness dependence: a) shows the short-circuit current density versus active layer thickness as predicted by the optical model, assuming an IQE of $100 \%$; b) shows the corresponding qualitative active layer thickness dependence of the open-circuit voltage, as obtained by electrical device simulations, for the case with a selective and a nonselective carbon contact. A thickness ratio of $2 / 3$ between $\mathrm{m}$-TiOx and $\mathrm{m}$-ZrOx layers (Figure 1a) within the optically active layer is assumed. The simulated current-voltage characteristics under 1 sun incident illumination are shown at an active layer thickness of c) $2000 \mathrm{~nm}$ and d) $200 \mathrm{~nm}$. The solid and dashed lines correspond to the cases with an external series resistance (from the electrodes and the external wires) of 0 and $10 \Omega \mathrm{cm}^{2}$, respectively. 

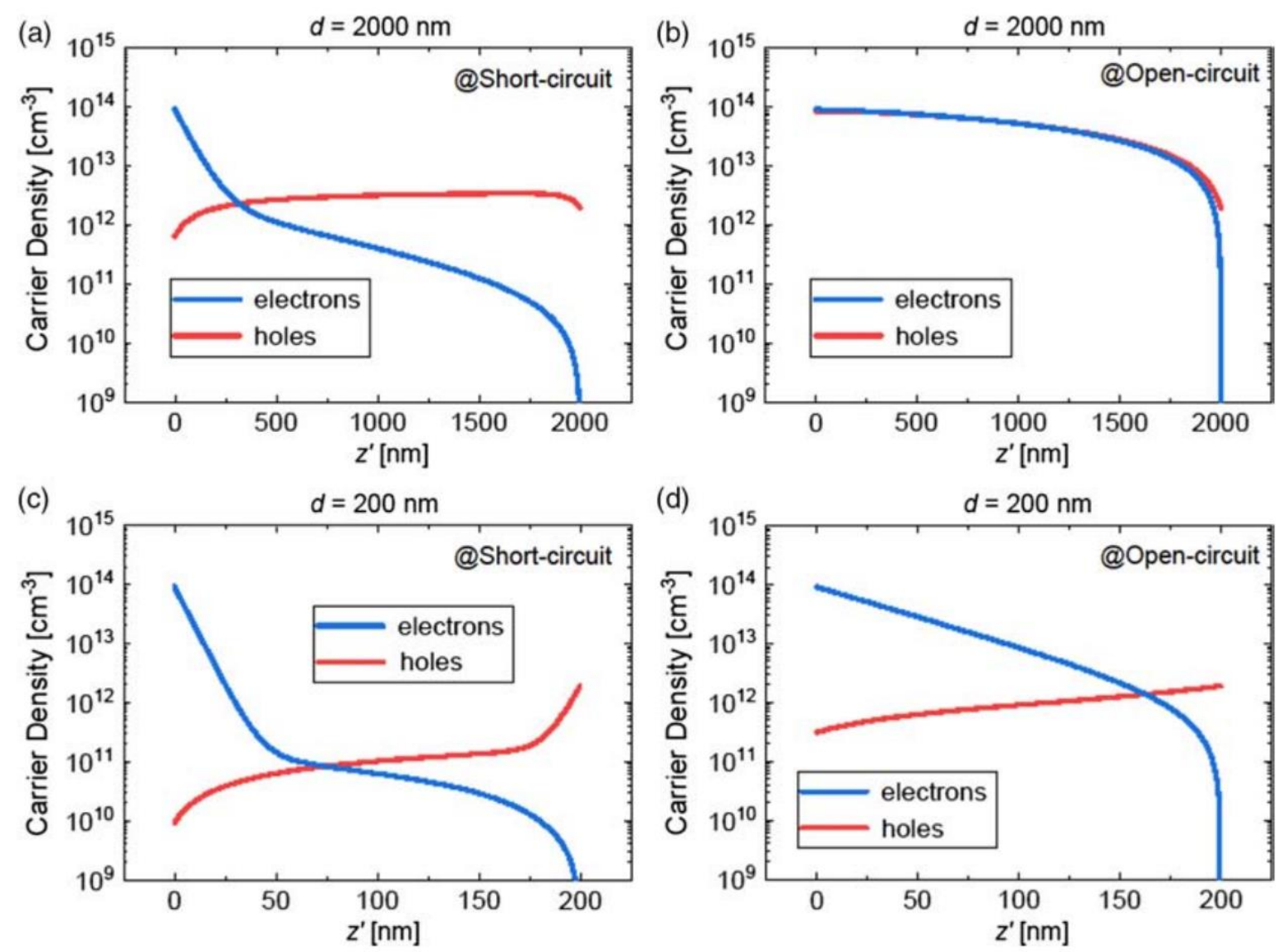

Figure 4.13. Simulated Charge Distribution For Carbon Cell

Figure 4.13 The charge carrier distributions inside the active layer under 1 sun incident illumination using the combined optical and electrical device model simulated at short-circuit and open-circuit $\left(z^{\prime}\right.$ being the linear distance inside the junction as measured from the transparent cathode) for the case with a nonselective carbon electrode. Here, the c-TiO2 contact is at $z^{\prime}=0$, whereas the nonselective carbon contact is at $z^{\prime}=d$. In all the electrical device simulations, an energy level structure defined by Figure $1 \mathrm{~b}$ is assumed. Furthermore, electron and hole mobilities of $10 \mathrm{~cm}^{2} \mathrm{Vs}^{-1}$, a dielectric constant of 13 , effective density of states of $10^{19} \mathrm{~cm}^{3}$, and second-order recombination with a coefficient of $6 \times 10^{11} \mathrm{~cm}^{3} \mathrm{~s} 1$ are assumed.

\subsection{Additional perovskite devices}

\subsubsection{Triple cation devices}

An in-depth discussion on the stability issues of perovskites, and more specifically triplecation perovskites with Cesium and Formamidinium and their reproducibility, can be found in Appendix A2. However, since this is more related to the experimental fabrication and detracts 
from the discussion on the opto-electronics of these devices, this discussion was kept as an Addendum.

\subsubsection{Perovskite silicon tandem cell}

Another interesting architecture that was examined and modelled more specifically for the purpose of investigating novel, more efficient devices was a tandem cell that incorporates both a perovskite and silicon active layer, and which can be optimized in layer thickness to absorb the wavelengths of light most relevant to each material to achieve efficiencies of over $29 \%{ }^{88,89}$. This system is challenging to simulate and a further robust test of the developed methodology. Furthermore, it is likely with the efforts of OxfordPV and others that the siliconperovskite tandem could be the first commercial implementation of the perovskite technology.
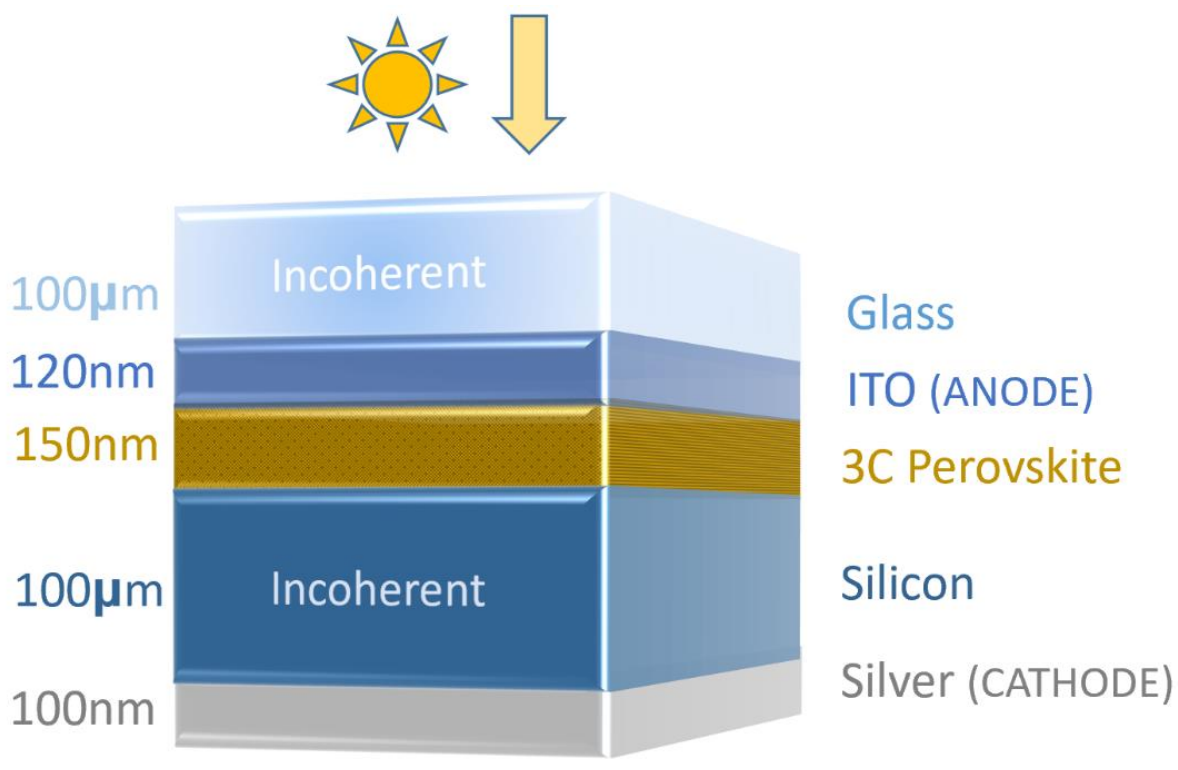

Figure 4.14 Perovskite Silicon Tandem Cell

Figure 4.14. Visual representation of the mesoporous carbon solar cell architecture as implemented in the initial optical model. In practice, various interlayers are added to facilitate a decrease in interfacial charge recombination, however the various interlayers may be subject to change and have thus been omitted for now.

In the tandem structure shown in Figure 4.14 , the perovskite junction is $150 \mathrm{~nm}$ thick and is the wider gap component of the stack (bandgap $1.55 \mathrm{eV}$, versus silicon at $1.1 \mathrm{eV}$ ). Optical constants that were used to model the 2 active layers of the stack can be found in Figure 4.15. From looking at the imaginary component $k$, the complementary nature of the two active 
materials, silicon and triple cation perovskite may be seen. Counter-intuitively, it will be shown that the silicon absorbs complementary light in the low energy regime of the spectrum, where its $k$ is small but non-zero. The perovskite is a triple cation blend with added Cesium and Formamidinium cations, fabricated as described by Saliba et al ${ }^{90}$. The optical constants of this particular blend were provided by this same group and independently verified with the Transmittance method using transmittance measurements of a sample on glass.
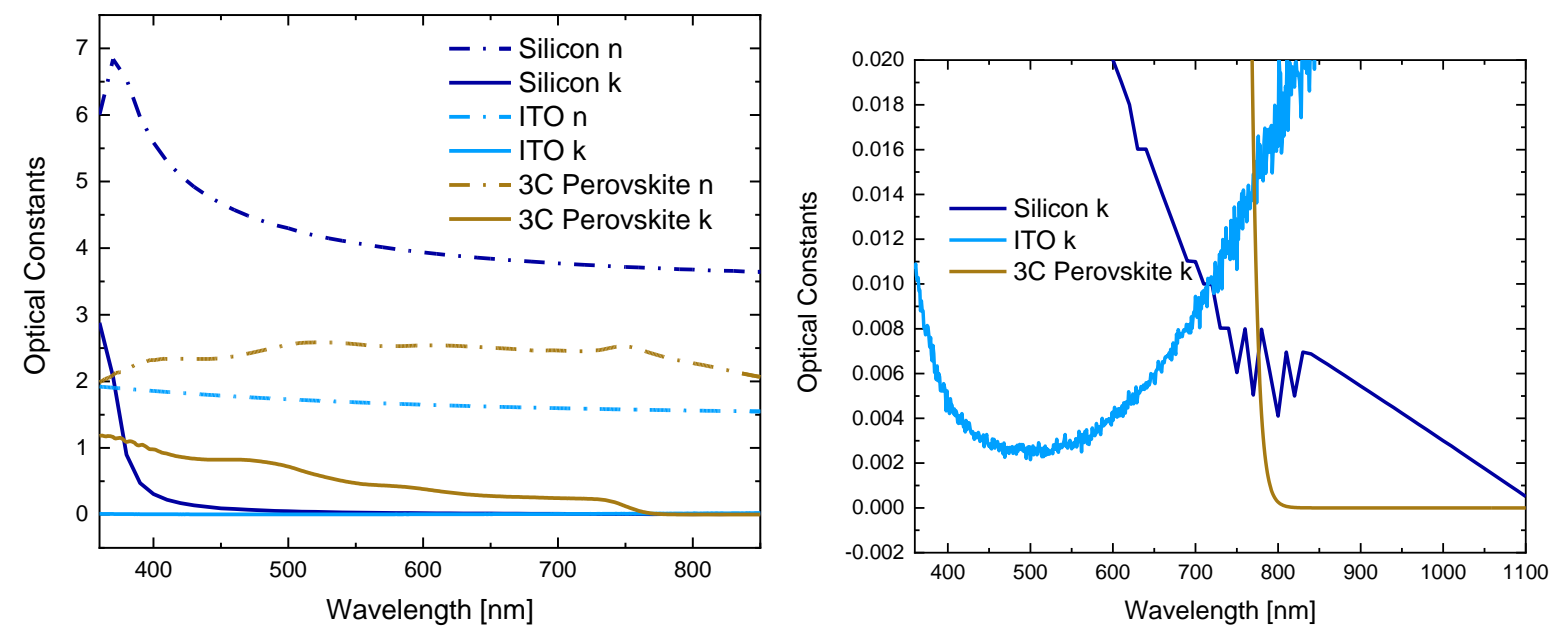

Figure 4.15. Optical Constants For Tandem Cell

Figure 4.15. Used optical constants of the active layers silicon and perovskite, as well as the ITO transparent electrode layer. On the right an enlarged depiction of the imaginary component $k$ is given for longer wavelengths. Due to the thickness of the silicon even this small imaginary component can cause significant absorptance.

A particular problem with modelling this type of tandem stack is that the silicon active layer is typically very thick, on the order of millimetres (which is possible due to much higher mobilities). This means that the silicon layer needs to be implemented as an incoherent layer, just like the glass substrate, in the optical transfer matrix simulation. Incoherence means interference in these layers is not coherent and thus de-coupled from the electric field interference in the coherent part of the stack (see Appendix A1 for an in-depth explanation on incoherent layers). Therefore, the presence of two decoupled layers significantly complicates the modelling efforts unless a decoupled matrix approach is used. A methodology doing exactly this is described by Harbecke et $\mathrm{al}^{47}$, and was implemented for the model. Figure 4.16 gives an overview of the internal fields in the stack and the spectral absorptance of the 
layers, while Figure 4.17 displays the generated charges in each active layer over the entire spectrum (modelled wavelength regime: 370-1150nm).

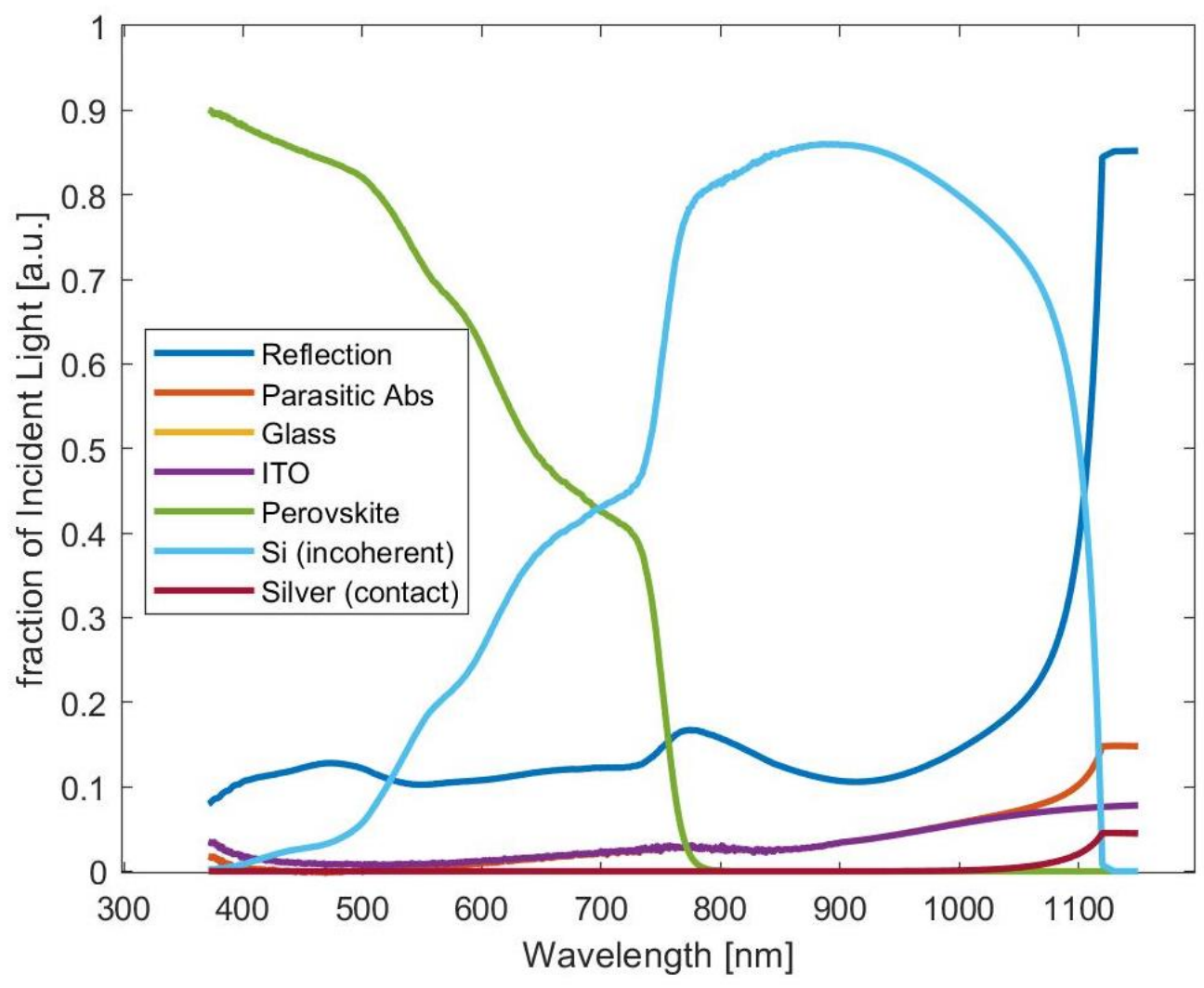

Figure 4.16. Spectral Absorption For Tandem Cell

Figure 4.16. Spectral Absorptance of a simplified version of the Tandem cell structure. Incoherent silicon layer can be seen to absorb most light past $800 \mathrm{~nm}$. (additional layers may be added to the coherent stack in practice to increase efficiency). Note that silicon mainly absorbs in the high wavelength (low energy) regime because the perovskite absorbs everything before that. 

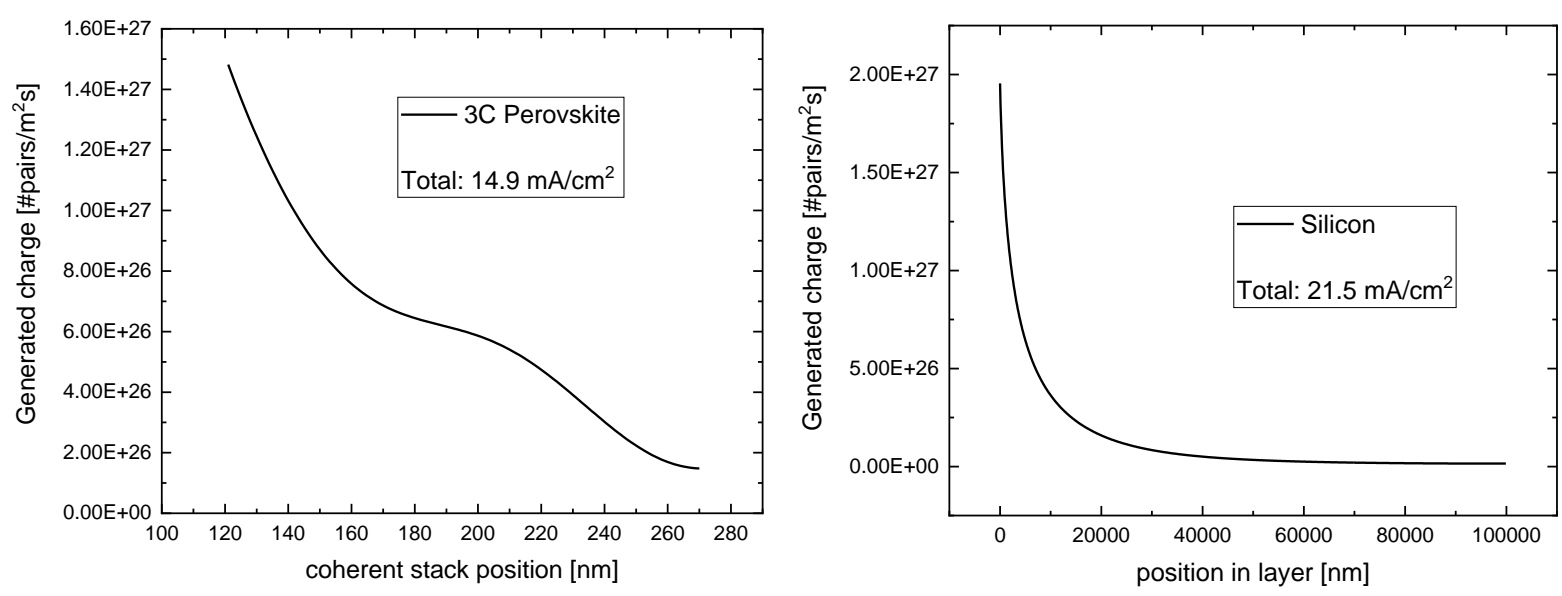

Figure 4.17 Generation Profile For Tandem Cell

Figure 4.17. Total amount of generated charges in each active layer. Left: the triple cation perovskite layer, showing coherent interference. Right: the silicon layer, showing near Lambert-Beer generation. Total generated current assuming perfect collection is also shown.

An additional challenge in this incoherent stack model was modelling the electric field in the coherent stack since the reflections from the incoherent layer must also be fed back as a scaling factor to the incident electric field to the coherent stack. For this a sum-series formula was used to calculate the infinite series of reflections of the glass layer and the reflections from the silicon layer:

$$
E_{\text {scaling factor }}=\frac{t_{\mathrm{AG}}}{\sqrt{1-\left|r_{\mathrm{coh}} r_{\mathrm{AG}}\right|^{2}}}
$$

Where $t_{A G}$ is the Fresnel transmission coefficient from air to glass, $r_{A G}$ the Fresnel reflection coefficient of the same boundary, and $r_{c o h}$ the total reflection coefficient from the coherent stack, assuming the silicon layer is semi-infinite. This latter part is an approximation in the sense that feedback from the silicon air interface reflection is neglected, but in practice this contribution to the coherent electric field is small (it can be quantified by the deviation of the parasitic absorptance from the expected value in Figure 4.17).

From the general trends observed in Figures 4.16 and 4.17, we can draw some preliminary conclusions about the optimization process. Firstly, the silicon layer will generally draw most of the low-energy light while the perovskite will draw most of the visible light and displays interference effects. When adding interlayers, it is therefore mainly the perovskite layer that 
must be optimized for the visible light spectrum. Secondly, the silicon is by far the biggest contributor to generated current, around $40 \%$ more than the perovskite.

\subsection{Conclusion}

Several third-generation solar stack architectures and their challenges were presented in this chapter, most importantly the carbon mesoporous stack and the perovskite-silicon tandem stack. Starting from the archetypal second-generation organic semiconductor system with PCDTBT:PCBM, we applied and demonstrated the utility of the methodology outlined in the previous chapters. The carbon stack architecture can be seen as a possible candidate for low cost, manufacturable perovskite solar cells. This is in part due to the possibility to have thick, micron-scale active layers, which is not possible with common (second-generation) organic cells. This thick active layer allows the architecture to make use of a cheap, non-selective carbon contact, and also allows the industrial screen-printing process to be used in fabrication. A full opto-electrical model was developed for the stack, and the external quantum efficiency of a previously empirically optimized operational device was simulated. Estimates of qualitative trends in active layer thickness were also made with Setfos to demonstrate the key bottlenecks and workings of the stack.

The tandem perovskite-silicon solar cell architecture was also modelled optically, without the inclusion of variable interfaces, although these can readily be added in an experimental optimization. The incoherent silicon layer was shown to absorb mostly in the low-energy regime, where the perovskite absorptance is zero, despite the low absorption coefficient of silicon here, which is due to its large thickness as an incoherent layer. It is therefore the perovskite layer that must be focused on in any optimization process. 


\section{Out-coupling model for active layer photoluminescence}

Measurements of both photo-and-electroluminescence (PL and EL) are extremely valuable for understanding voltage losses and thermodynamic limits in photovoltaic devices. Particularly when considering sub-gap dynamics such as CT state and trap energies, the relative optical 'thin-ness' of both organic solar cells and to a certain extent, homojunction perovskite devices requires one to carefully consider interference effects in both EL and PL. In this chapter an approach is advanced to account for these effects to reconstruct and predict the luminescence characteristics of thin film structures. The method has utility in not only reciprocity analysis in solar cells but also in modelling light emitting diodes.

\subsection{General outline}

\subsubsection{Experimental introduction to luminescence}

While the previous chapter focused mainly on the class of perovskite solar cells, some experimental was also performed on non-fullerene devices that might serve as an introduction to the luminescence subject. In addition to optical constants, a photo-excited free carrier recombination study was performed on PM6:Y6, one of the best-performing nonfullerene blends at the time of analysis ${ }^{91}$. At the time of writing there are already better performing blends, such as the PM6:Y6:BTP-M ternary cell ${ }^{92}$, which demonstrates how quickly the field of non-fullerenes is growing.

Transient photoluminescence spectroscopy was used to measure the recombination lifetimes of the PM6:Y6 blend (1:1.2) using an ultrafast camera setup with a 550nm monochromatic laser which was shuttered to irradiate the sample after which emission was recorded in order to quantify recombination rates. This was done firstly for the neat materials on glass and secondly for the blend, to quantify the existence of quenching in the blend. The $550 \mathrm{~nm}$ laser source drives excitation of excitons, which then recombine (on a timescale of picoseconds) and emit at a higher frequency matching the respective energy difference of the state transition. This can be the HOMO-LUMO difference (usually the highest intensity) but also the charge transfer state energy (see Figure 1.3 in Chapter 1), depending on the exciton relaxation 
pathway. The HOMO-LUMO energy difference (and sometimes the CT state energy difference too, though this signal is much weaker) can be determined from the observed emission peaks in steady-state PL. The peak for PM6 was determined at 700nm (or $1.77 \mathrm{eV}$ ) and that of $\mathrm{Y} 6$ at $860 \mathrm{~nm}$ (or $1.44 \mathrm{eV}$ ). Note that this only gives an estimate for the energy difference but not the absolute HOMO or LUMO levels.

The next step in transient PL spectroscopy is to measure the intensity of the emission after excitation over time, which gives an idea of how quickly the excitons recombine. Given in Figure 5.1 is exactly this, with the emission scaled logarithmically to give a clearer fit with the lifetime decay equation ${ }^{93}$ :

$$
I_{P L, r e l}=A \cdot e^{-\frac{t}{\tau}}
$$

Where $I_{P L, r e l}$ is the measured photoluminescence intensity, $A$ some proportionality constant, $t$ the time variable and $\tau$ the lifetime which we want to fit for. As can be seen in Figure 5.1, the neat Y6 has a longer lifetime (in the range of 27ps) than the neat PM6 (in the range of $2 p s)$. The interesting part is however to look at the blend, and we can see that the respective lifetimes of both materials and their induced excitons are significantly quenched. This implies that both absorptance pathways for the acceptor and donor material are contributing channels to the charge collection in the cell. 


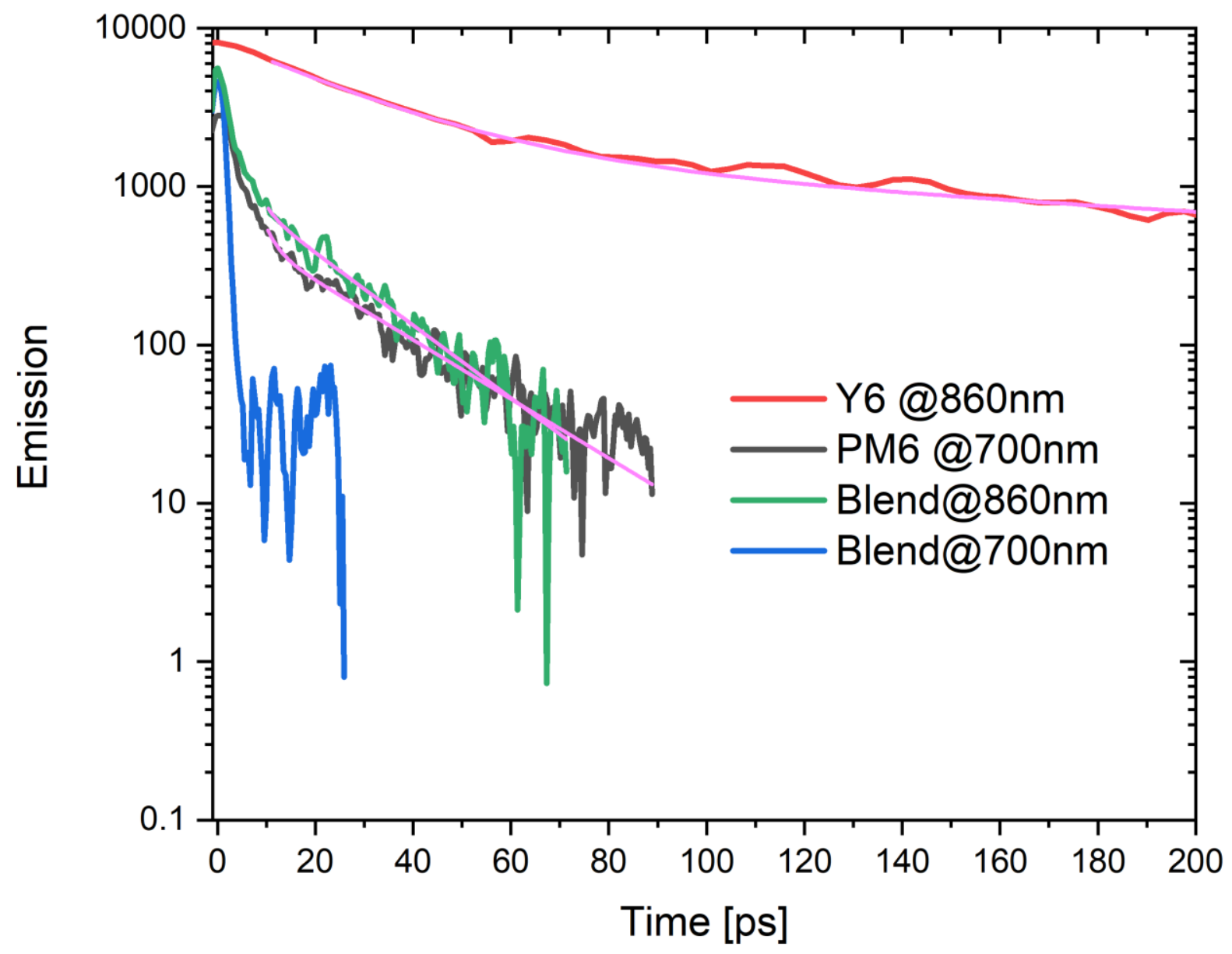

Figure 5.1 Transient PL on PM6:Y6

Figure 5.1. Transient PL measurements and their fit with lifetime formula. Fit values for the respective materials at the relevant wavelengths were: $\tau_{\mathrm{PM} 6,700 \mathrm{~nm}}=2.4 \mathrm{ps}(700 \mathrm{~nm}), \tau_{\mathrm{Y} 6,860 \mathrm{~nm}}$ $=27.2 \mathrm{ps}, \tau_{\text {Blend, } 860 \mathrm{~nm}}=2.1 \mathrm{ps}, \tau_{\text {Blend, } 700 \mathrm{~nm}}=$ no fit, strongly quenched. The blend PL being immediately quenched at 700nm indicates very efficient exciton dissociation for excitons generated in the PM6.

The efficient exciton dissociation in both materials of the non-fullerene blend has raised some question in regard to the energetic pathways that drive this dissociation, in particular because the energetic off-set between their respective HOMO levels is quite small for a wide range of non-fullerenes, including the PM6:Y6 ${ }^{91}$. It is of particular interest because, unlike fullerenes, the acceptor in non-fullerenes also absorbs a significant part of the solar spectrum ${ }^{94}$, making the cell effectively act as a sort of blended tandem structure (another example of a tandem structure would be the perovskite-silicon tandem cell previously discussed, with similarly improved efficiencies). A recent review was published discussing the particular nature of non- 
fullerenes and the role of the two separate charge collection channels (one for the acceptor and one for the donor) ${ }^{95}$.

\subsubsection{Luminescence fitting in thin-films}

While the previous experiment showcases some information on carrier lifetime and HOMO LUMO differences, it tells us very little about the CT state of the examined material. Luminescence of an opto-electrical material can be used not only in methods such as transient-PL, but also to extract useful parameters about the CT state parameters in blends by fitting a spectral measurement of emission to theoretical models such as Marcus theory, which builds on the reciprocity principle. In the case of Marcus theory, the extracted parameters are the energy of the $\mathrm{CT}$ state $\left(E_{\mathrm{CT}}\right)$, reorganization energy $\left(\lambda_{\mathrm{CT}}\right)$, and oscillator strength $\left(f_{\sigma}\right)$ related to the electronic coupling, as well as emission probability $f_{1}$. Ideally, the absorption cross-section $\left(\sigma_{C T}\right)$ and emission $(I)$ are related through reciprocity so that

$$
\begin{aligned}
\sigma_{\mathrm{CT}} & =\frac{f_{\sigma}}{E \sqrt{4 \pi \lambda_{\mathrm{CT}} k_{\mathrm{B}} T}} \exp \left(-\frac{\left(E_{\mathrm{CT}}+\lambda_{\mathrm{CT}}-E\right)^{2}}{4 \lambda_{\mathrm{CT}} k_{\mathrm{B}} T}\right) \\
I & =\frac{f_{I} E}{\sqrt{4 \pi \lambda_{\mathrm{CT}} k_{\mathrm{B}} T}} \exp \left(-\frac{\left(E_{\mathrm{CT}}+\lambda_{\mathrm{CT}}-E\right)^{2}}{4 \lambda_{\mathrm{CT}} k_{\mathrm{B}} T}\right)
\end{aligned}
$$

where $k_{\mathrm{B}}$ is the Boltzmann constant, $T$ the absolute temperature, and $E$ the photon energy ${ }^{96}$. However, when fitting these parameters for thin-films such as those characteristic of high efficiency organic solar cells, there are known to be errors in the fit depending on the active layer thickness. Experimental evidence that this effect is significant was previously published by our group, Armin et $a^{96}$.

As has been argued before by independent groups 57 , the problem with thin-film luminescence measurements is that this distortion occurs due to the significant effect of thinfilm interference on the luminescence out-coupling. This means that thin-film layer emission measurements cannot in good faith be fitted to Marcus theory, at least not when measured and fitted directly without any compensation for the cavity effects.

\subsubsection{An approach to account for cavity effects}

As discussed previously, thin-film layers, particularly when they reside within a partially reflecting cavity, suffer from interference effects that can drastically alter the internal electric 
field within the layer. As a solution to this one might imagine simply taking a thick layer measurement of the same material and fitting the emission. However, for some organic semiconductors of relevance to the work described in this thesis, such as PM6, if the layer is made too thick (>300 $\mathrm{nm}$ ), the optical constants (and thus absorption cross-section) may be modified versus the thin film limit due to morphological impacts (this was experimentally observed with ellipsometry and transmittance measurements and reported in Chapter 3). The same could also be said for certain organic-inorganic perovskites where, for example, relative differences in the solvent evaporation rate and crystallisation come into play.

As described in previous chapters, the interference modified electric field in thin-film layers can be modelled with the help of techniques such as FDTD or the transfer matrix method for the case of plane wave coupling into a cavity. The question arises as to whether a mirror equivalent output coupling model based upon the same principles could be developed.

Such a model would require several key elements:

- The internal field must be calculated to obtain the generated charge profile. This has already been discussed in previous chapters.

- The drift-diffusion (5.2.1) of generated charge must be considered and compared to the lifetime of the charges. If the generated charge profile changes significantly due to charge movement before charges recombine (and thus emit), this also has to be modelled.

- The re-absorption and re-emission (5.2.2) must be considered.

- The outcoupling (5.2.3) radiation will again undergo interference effects.

\subsection{A thin-film emission model}

The model simulates the relative photon emission of an active layer on a glass substrate, illuminated by a laser, and detected at a certain angle from the opposite side i.e. the process of photoluminescence from an emissive thin film as a function of angle. Off-normal collection of the emission provides a means to reject the incident radiation. The experimental set-up was shown in Figure 1.12 but is shown again in Figure 5.2 for clarity with some more extensive details. 


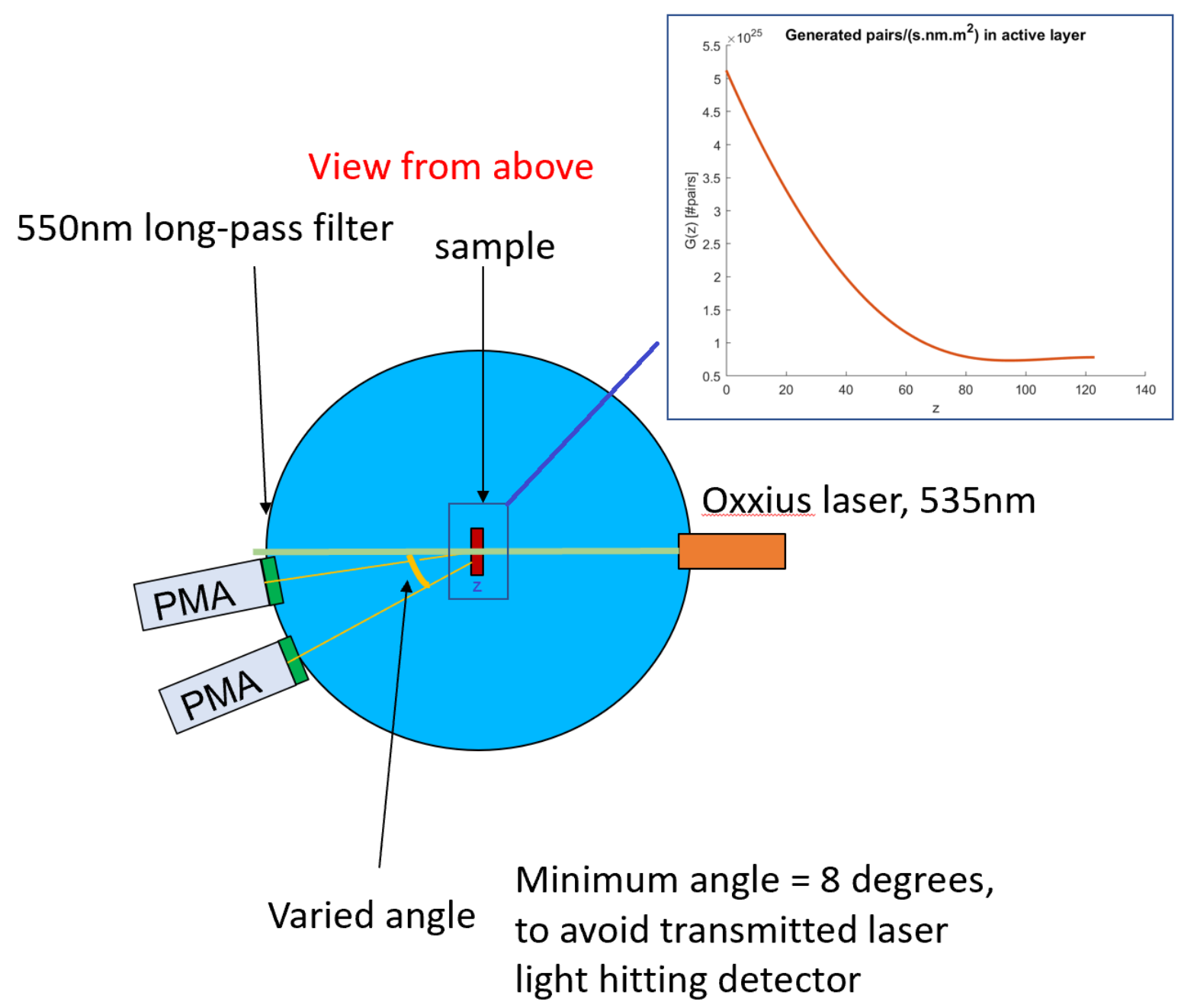

Figure 5.2 Rotating Emission Set-Up (Detailed)

Figure 5.2. Rotating set-up for measuring photoluminescence on an organic semiconductor thin film sample on glass. A Photonic Multichannel Analyzer (PMA-12) is used to detect the relative number of emitted photons at a certain angle and can be rotated around the stationary sample. Inset shows the interference pattern of generated charges in sample.

We will talk about relative photon emission because the detection also happens within a certain solid angle, which will affect the measured intensity. Additionally, there is the loss due to other factors such as scattering and light emitted by out-coupling from the sample sides, all of which are difficult to model absolutely in terms of measured photons (Figure 5.3 gives an overview of what is meant by these losses). 


\section{sample}

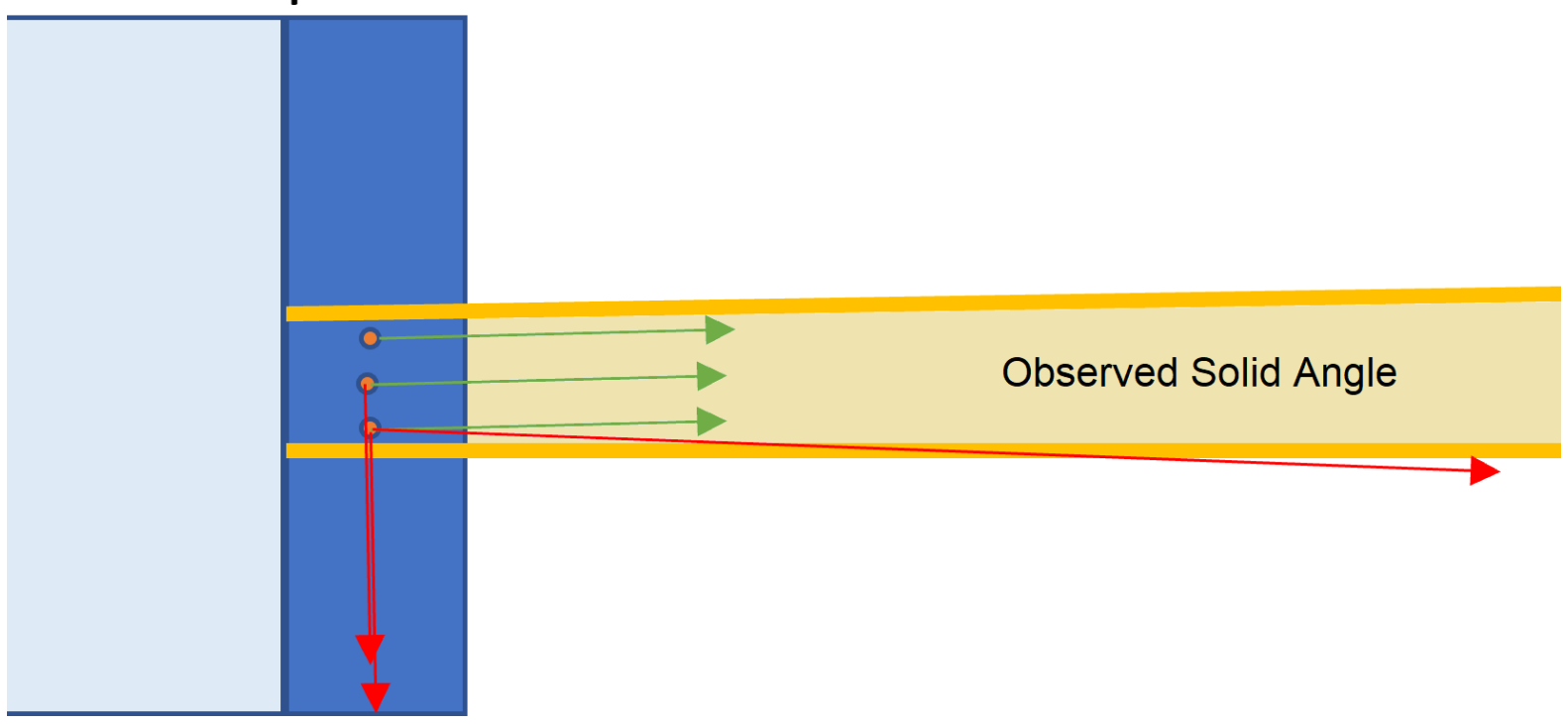

Figure 5.3. Plane Wave Approximation

Figure 5.3 The losses caused by observing emission only within a certain solid angle. Green arrows signify emitted light coming from the dipoles that is observed, red arrows are emitted light that is not observed. By approximation, as seen from this figure, observed light will behave as parallel plane waves.

We will assume for now that these losses are not significantly changed by varying film thickness or different optical constants of the active layer material (in other words we assume the main reason for distortion between PL or EL measurements of different materials is the interference effect). In practice only the out-coupling from the sample sides will have an effect that changes with layer thickness, because for this light the sample can act as a waveguide due to its small thickness. Comparisons with Setfos, which does model this, will show that this effect is still quite negligible.

If the optical constants and thickness of our active layer are known (using measurement techniques discussed in previous chapters), we can account for the interference effect and scale our measurements accordingly, so a sense of genuine comparison between different materials can be gained. The ultimate goal here would be to measure and simulate interference-corrected, steady-state photoluminescence as a function of wavelength, averaged over measurements at several thicknesses. From this, a legitimate or at least more 
accurate Marcus fit could then be obtained, giving insight in all the useful unknown parameters involved.

\subsubsection{Drift-diffusion model}

While for organics semiconductors mobility generally tends to be low relative to inorganic semiconductors (around $3.6 \times 10^{-3} \mathrm{~cm}^{2} \mathrm{~V}^{-1} \mathrm{~s}^{-1}$ for PCDTBT ${ }^{97}$ ), it is not a given that generated charges will recombine before drift-diffusing significantly, which would mean the recombination profile looks different from the generation profile.

For this reason, a drift-diffusion model was implemented in Matlab, using a simplified version of the current and continuity equations as already discussed in Chapter 2. No transverse electric field is present because excitons are neutral, and the drift term is therefore ignored:

$$
-D_{e} \frac{\delta^{2} e(x)}{\delta x^{2}}=G_{e}(x)-\frac{e(x)}{\tau}
$$

$e(x)$ is the positional exciton concentration per $\mathrm{m}^{2}, D$ is the diffusion coefficient, and $G_{e}(x)$ and $\frac{e(x)}{\tau}$ are the positional generation and recombination of free carriers in the layer. It is possible to solve this differential equation analytically if we make use of the transfer matrix method to calculate $G(\mathrm{x})$. Recall that the internal electric wave is a superposition of a forward travelling wave $E^{+}(\mathrm{x})$ and a backwards-travelling wave $E^{-}(\mathrm{x})$, which are both plane waves. The amplitude of both waves has to be calculated with transfer matrix, but the dependency on position $x$ is always the same:

$$
\begin{gathered}
E^{+}(x)=E^{+} \exp \left(i \cdot \frac{2 \pi \overline{n_{l}}}{\lambda} x\right) \\
E^{-}(x)=E^{-} \exp \left(-i \cdot \frac{2 \pi \overline{n_{l}}}{\lambda} x\right)
\end{gathered}
$$

Giving a differential equation that can be perfectly solved with certain boundary equations. Typically, in the literature, the boundary conditions of a layer bounded by insulators (glass and air) are taken as $\frac{d e(x)}{d x}=0$, at the edges of the layer $x=0$ and $x=L{ }^{55}$. This is justified in by noting that the diffusion current at the edges will necessarily be zero at boundaries with nonconductive material (glass/air). 


$$
\left.D_{e} \frac{\delta^{2} e(x)}{\delta x^{2}} d x\right|_{x=0, L}=0
$$

Which would then simplify to $\left.\frac{\delta e(x)}{\delta x}\right|_{x=0, L}=0$. However, this is not necessarily a given since the real reason there is no diffusion current flowing into the adjacent material is because the mobility (and with it, $D$ ) goes to zero beyond that boundary, and not because the gradient in carrier concentration is zero at the boundary (this might not be the case). The real reason this boundary condition is valid is because in an isolated layer in steady state, the total integrated generation $\int_{0}^{L} G(x)$ must equal the total integrated recombination $\int_{0}^{L} \frac{e(x)}{\tau}$, which means according to Eq. 5.2, that $\int_{0}^{L} D_{e} \frac{\delta^{2} e(x)}{\delta x^{2}} d x=0$. From this, indeed, the accepted boundary condition follows. Figure 5.3 shows the effect of the boundary condition as compared to an arbitrary one $\left(\left.\frac{\delta^{2} e(x)}{\delta x^{2}} d x\right|_{x=0, L}=0\right.$, which would follow from the justification in literature), as well as a quenching boundary condition $\left(\left.e(x)\right|_{x=0, L}=0\right)$ which would be the case if the layer was in contact with a metal or a different quenching material.

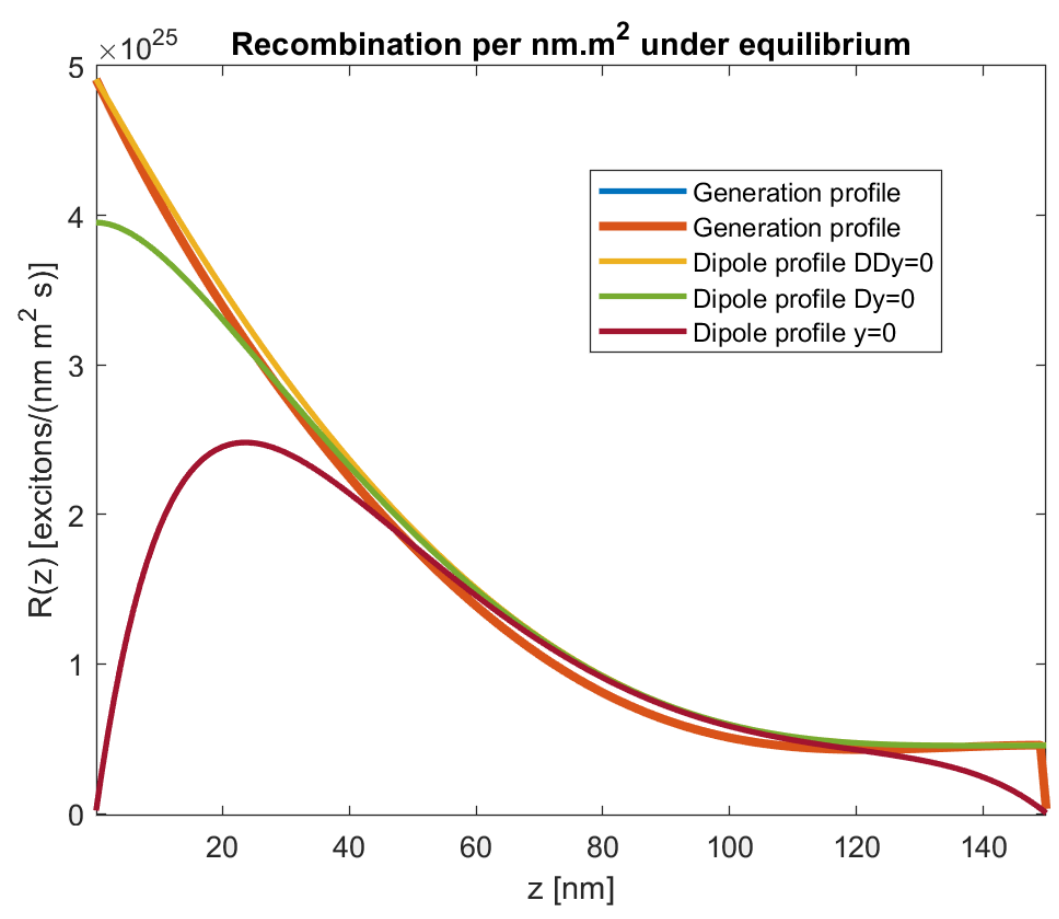

Figure 5.4. Boundary Conditions For Diffusion Profile

Figure 5.4. A comparison of boundary conditions for solving the simplified continuity equation 5.2. D stands for $\frac{\delta e(x)}{\delta x}$ and $\mathrm{y}$ for $e(x)$. Diffusion length is set at $13 \mathrm{~nm}$. 
As mentioned in Chapter 2, certain values must also be assumed for both $\tau$ and $D_{e}$, depending on the active layer material. They can be combined in one free value, the diffusion length $L_{d}$ :

$$
L_{d}=\sqrt{\tau D_{e}}
$$

Since the experiments were performed on the archetypal organic semiconductor PCDTBT (a neat organic semiconducting material, which means we are dealing with excitons), we will use the literature value for this diffusion length (around $10 \mathrm{~nm}{ }^{98}$ ). Figure 5.5 shows a comparison of various diffusion lengths.

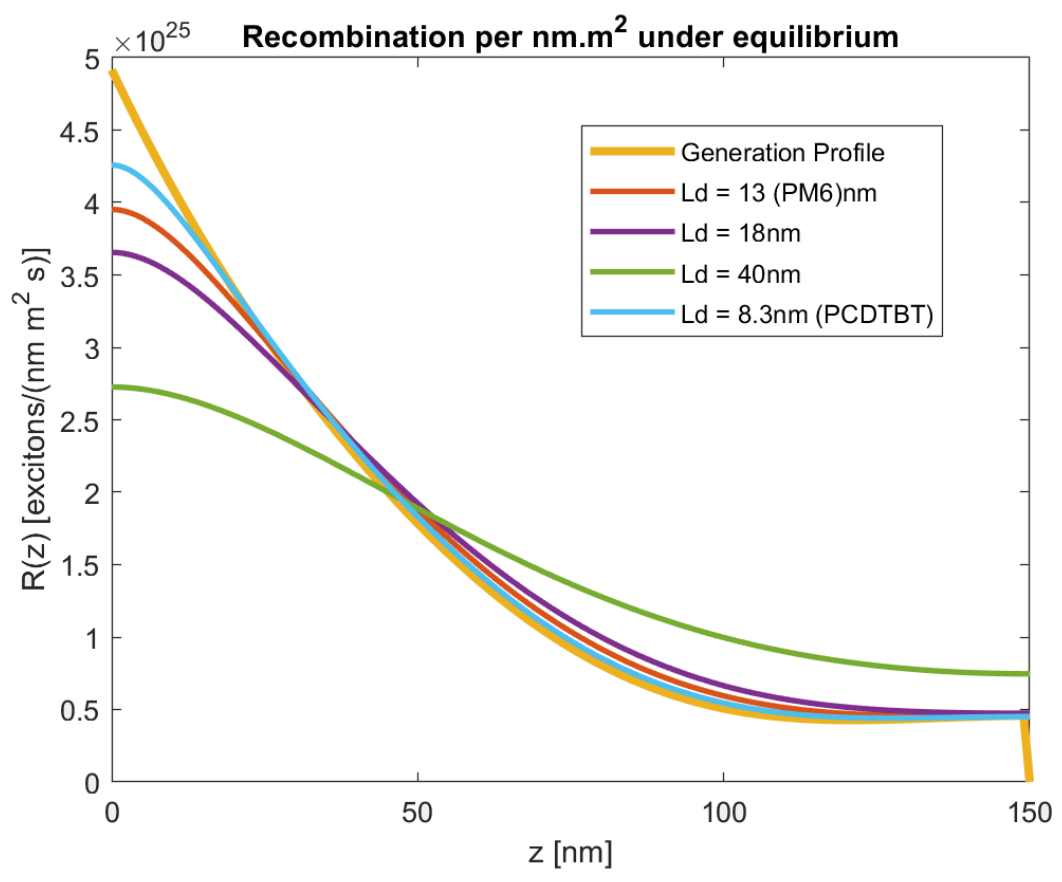

Figure 5.5. Diffusion Lengths Impact

Figure 5.5. Comparison of the effect of different diffusion lengths on the dipole profile (and thus recombination rate profile), calculated with the exciton continuity equation.

Indeed, we see that for the typical organic semiconductor, PCDTBT, the dipole profile will be quite similar to the generation profile. However, for other materials this may not be the case, as the significant change can be seen around $L_{d}=40 \mathrm{~nm}$. It will therefore be important to check this value for each examined material before proceeding with emission models for spectral fitting. 


\subsubsection{Reabsorption and re-emission}

One might imagine, looking at Figure 5.2, that the propagation of the emission in the stack can be modelled in similar fashion as the transfer matrix method, with a forwards- and backwards-propagating plane wave that consists of the sum of all separate plane waves. In literature, a different model than plane wave is used to calculate the out-coupling after determining the dipole profile. Some of the common approaches are FDTD ${ }^{99}$, scattering matrix method ${ }^{57}$, or, in the case of the commercial software Setfos, Green's functions that model many dipoles interacting with each other ${ }^{50}$.

The scattering matrix method improves on FDTD, which can show spurious effects of numerical dispersion, but it can be very resource-intensive and is not easy to generalize to models for novel types of opto-electronic structures and materials. The problem with the interacting dipoles model approach (used by Setfos) is that reabsorption (and therefore reemission) of the emitted light is not modelled.

While using a plane wave model necessarily has some approximations, it does easily lend itself to model the reabsorption of the emitted light (and here in fact it has an advantage over the Green's function dipole model). The fraction of light that is re-emitted, however, is a free parameter that will turn out to become quite significant for thicker samples. We can define this free parameter as $P_{r}$, the probability of radiative recombination for an exciton. Usually in literature this probability is determined using rates of the available pathways. In a simplistic model where there are only two rates considered, $k_{r}$ the rate for radiative recombination and $k_{n r}$ the rate for non-radiative recombination, it would equal:

$$
P_{r}=\frac{k_{r}}{k_{r}+k_{n r}}
$$

Were this parameter known, it would be possible to model the re-emission with iterative steps, each time calculating absorption, applying the continuity equation if needed for the step's dipole profile, and then reducing the intensity of that dipole profile by $P_{r}$.

Even this treatment isn't fully adequate as real emission also undergoes a smeared-out Stokes shift (see Figure 5.6) in wavelength, so some excitons will recombine and emit at different frequencies than the modelled ones. In principle a Stokes shift could be measured and also implemented in the model. 


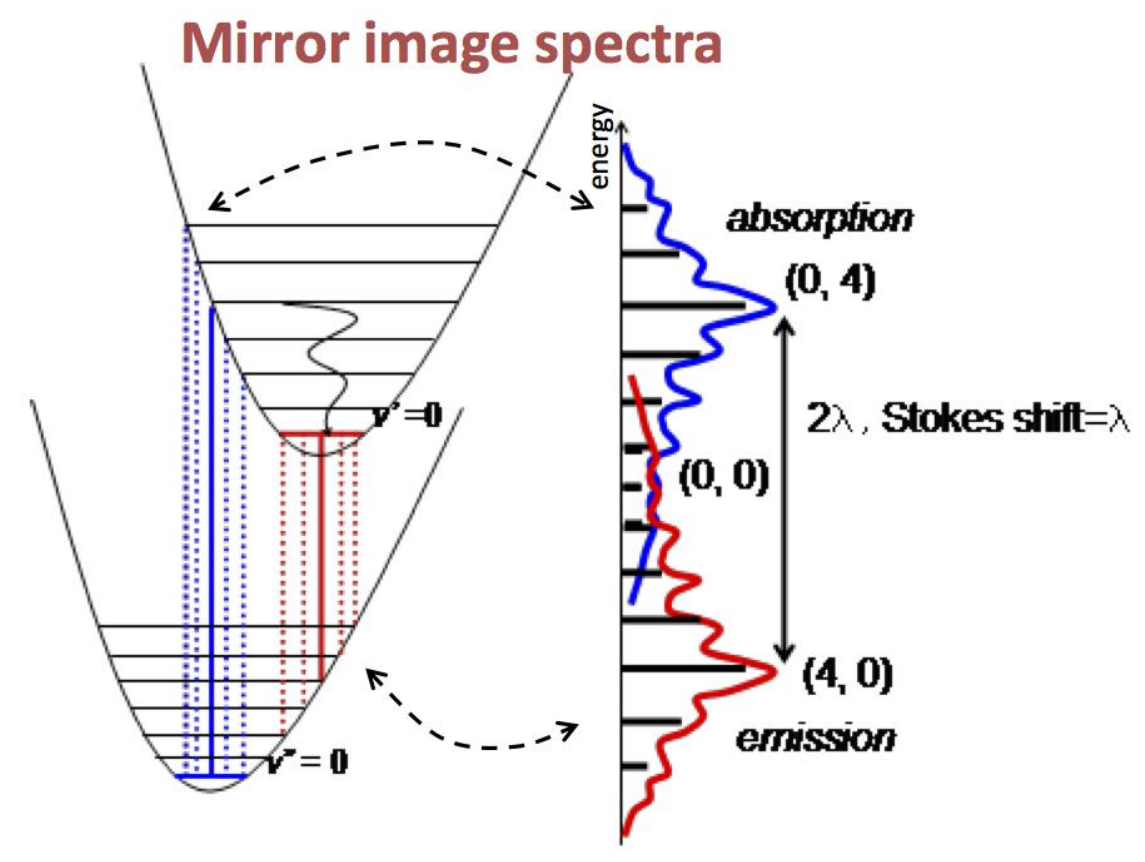

Figure 5.6. Stokes Shift

Figure 5.6. Stokes shift visualized through its underlying principle of vibrational energy levels around the principle energy levels. The shift is due to vibrational relaxation. Modified upon reproduction from. ${ }^{100}$

For the current implementation of the model, the amount of reabsorption and re-emission will be assumed to be very small compared to initial absorption and emission and be neglected, but measurements could in principle be undertaken to determine this parameter.

\subsubsection{Out-coupling model}

Compared to the FDTD model and the Green's function model, the plane wave out-coupling approach approximates the other methods in two notable ways:

1. Dipole emission is in all directions. Losses from the sides of the active layer (as visualized by the downwards red arrows in Figure 5.2) are not modelled by the plane wave model. The first approximation is that the amount of light coupled into these "evanescent modes" does not change significantly with thickness. In reality, there may be more or less outcoupling depending on thickness, since layer thickness will act as if affecting waveguide dimensions, in certain directions, depending on which modes can form in transverse direction. However, as indicated above from comparisons with 
Setfos calculations, this thickness-related effect is rather insignificant, because the evanescent modes generally account for a small fraction of the emission.

2. Surface dipole effects are not accounted for. This is a more serious impact caused by an antenna effect ${ }^{50}$ close to the layer surface. For these dipoles, optical feedback with the surface causes increased recombination, but almost exclusively radiating through evanescent modes (see Figure 5.6). Effectively for our plane wave model, it means that emission near surfaces is heavily quenched. However, this effect can be approximated mathematically by calculating a dipole profile with boundary conditions set to a quenching material at the interface instead (see Figure 5.3, y=0, and compare to Figure 5.7). We will call this extra approximation the Quench model.

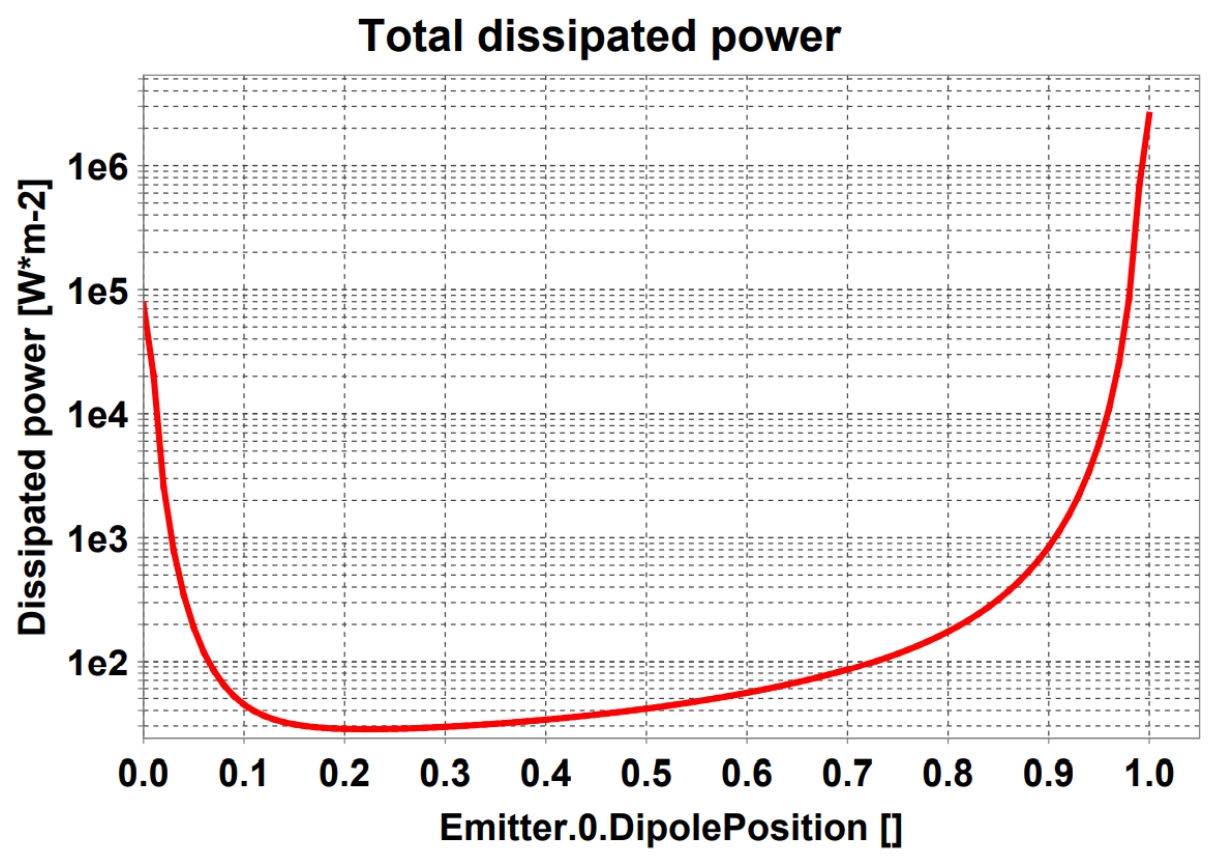

\section{Total dissipated power}

Figure 5.7. Boundary Emission Feedback

Figure 5.7. Modified upon reproduction from the Setfos 5.0 manual: "dynamics of dipoles is enhanced close to the electrodes due to strong optical feedback. Only, as mode analysis shows, the "additional" dissipated power is mostly coupled to evanescent modes, instead of being emitted out of the device. On the other hand, the increase of the radiative decay rate for dipoles close to the electrodes results in a strong reduction of the exciton density at those positions: we call this effect exciton quenching." 
Aside from these approximations the plane wave model will be shown to yield similar results to the Setfos model. The out-coupling interference effect is modelled by assuming, again, a forward propagating and a backward propagating wave. However, at every point $x$ in the stack, we will now have a backward and a forward propagating plane wave originating from that point, due to exciton recombination at that location.

Let us call $E_{D I P}$ the electric field caused by the initial radiative exciton recombination, named $D I P$ because the excitons act as radiating dipole antennas. $E_{D I P}$ is a known from the exciton profile in the relative sense (compared to other locations in the layer), since it is directly proportional to the square root of the recombination rate $R(x)$ at $x$, following Eq. 3.17.

$$
R(x)=\frac{e(x)}{\tau} \approx \frac{c \eta \varepsilon_{0}}{2}\left|E_{D I P}(x)\right|^{2}
$$

Now we need to account for the interference effect on $E_{D I P}(x)$ resulting from reflections off the layer interfaces. The forwards-propagating wave, $\boldsymbol{E}_{*}^{+}(\boldsymbol{x})$, has to be calculated as a sum of all contributing reflections. This can be done with a series/sum manipulation, which is visualized for a PCDTBT layer in figure 5.8:

$$
\begin{aligned}
\boldsymbol{E}_{*}^{+}(x) & =\frac{1}{2} \frac{1}{\Omega} E_{D I P}^{+}(x) \cdot\left[1+e^{\frac{2 \pi \bar{n}}{\lambda}\left[2 t_{1}+2 t_{2}\right]} \cdot r_{P G} r_{P A}+e^{\frac{2 \pi \bar{n}}{\lambda}\left[4 t_{1}+4 t_{2}\right]} \cdot\left(r_{P G} r_{P A}\right)^{2}+\cdots\right] \\
& +\frac{1}{2} \frac{1}{\Omega} E_{D I P}^{-}(x) \cdot\left[e^{\frac{2 \pi \bar{n}}{\lambda}\left[2 t_{1}\right]} \cdot r_{P G}+e^{\frac{2 \pi \bar{n}}{\lambda}\left[4 t_{1}+2 t_{2}\right]} \cdot r_{P G}{ }^{2} r_{P A}+\cdots\right]
\end{aligned}
$$

Where $\Omega$ is the solid angle, $E_{*}^{+}(x)$ the total forwards propagating emission wave at $x$, and $t_{1}$ and $t_{2}$ the respective distances to both layer interfaces at $x . r_{P A}$ and $r_{P G}$ are the Fresnel reflection coefficients for the PCDTBT/Air and PCDTBT/Glass interfaces, $\bar{n}$ the (complex) refractive index and $\lambda$ the wavelength of the emitted light. The formula is colour-coded to the figure to help in the visualization. 


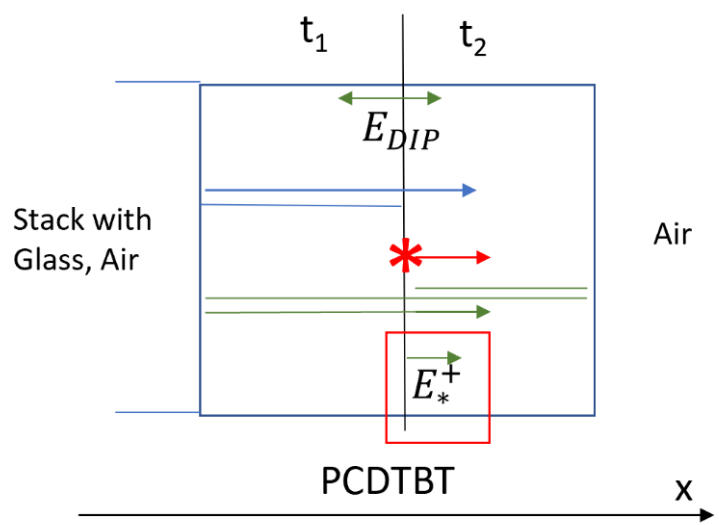

Figure 5.8. Plane Wave Model Interference

Figure 5.8. Visualization of the contributions to the forwards field in the active layer, $E_{*}^{+}(x)$.

This series can then be rewritten as an infinite sum:

$$
\begin{aligned}
& \boldsymbol{E}_{*}^{+}(x)=\frac{1}{2} \frac{1}{\omega} E_{\mathrm{DIP}}^{ \pm}(x) \cdot \sum_{m=0}^{\infty}\left(e^{\frac{2 \pi \bar{n}}{\lambda} 2\left[t_{1}+t_{2}\right]} \cdot r_{\mathrm{PG}} r_{\mathrm{PA}}\right)^{m}+ \\
& \frac{1}{2} \frac{1}{\omega} E_{\mathrm{DIP}}^{ \pm}(x) \cdot e^{\frac{2 \pi \bar{n}}{\lambda}\left[2 t_{1}\right]} \cdot r_{\mathrm{PG}} \sum_{m=0}^{\infty}\left(e^{\frac{2 \pi \bar{n}}{\lambda} 2\left[t_{1}+t_{2}\right]} \cdot r_{\mathrm{PG}} r_{\mathrm{PA}}\right)^{m}
\end{aligned}
$$

Which can again be rewritten using the following mathematical manipulation, which is valid for infinite sums with factors $F$ between 0 and 1 :

$$
\begin{gathered}
\sum_{m=0}^{\infty}(F)^{m}=S_{m} \\
F . S_{m}=S_{m+1} \\
(1-F) S_{m}=1-F^{m+1} \\
S_{m}=\frac{1-F^{m+1}}{1-F}
\end{gathered}
$$

Where $F^{m+1}$ becomes 0 for $m \rightarrow \infty$. Consequently, we can rewrite Eq. 5.9b as:

$$
\begin{aligned}
\boldsymbol{E}_{*}^{+}(x)= & \frac{1}{2} \frac{1}{\omega} E_{\mathrm{DIP}}^{ \pm}(x) \cdot \frac{1}{1-e^{\frac{2 \pi \bar{n}}{\lambda} 2\left[t_{1}+t_{2}\right]} \cdot r_{\mathrm{PG}} r_{\mathrm{PA}}}+ \\
& \frac{1}{2} \frac{1}{\omega} E_{\mathrm{DIP}}^{ \pm}(x) \cdot e^{\frac{2 \pi \bar{n}}{\lambda}\left[2 t_{1}\right]} \cdot r_{\mathrm{PG}} \frac{1}{1-e^{\frac{2 \pi \bar{n}}{\lambda} 2\left[t_{1}+t_{2}\right]} \cdot r_{\mathrm{PG}} r_{\mathrm{PA}}}
\end{aligned}
$$




$$
=\frac{1}{2} \frac{1}{\omega} E_{\mathrm{DIP}}^{ \pm}(x) \cdot \frac{1+e^{\frac{2 \pi \bar{n}}{\lambda}\left[2 t_{1}\right]} \cdot r_{\mathrm{PG}}}{1-e^{\frac{2 \pi \bar{n}}{\lambda} 2\left[t_{1}+t_{2}\right]} \cdot r_{\mathrm{PG}} r_{\mathrm{PA}}}
$$

Which is our final equation for $\boldsymbol{E}_{*}^{+}(x)$. Note that this only calculates interference between the backwards and forwards emission from one position $\mathrm{x}$. For the full emission that is observed, $E_{\text {out,Total }}$, we need to phase shift all $\boldsymbol{E}_{*}^{+}(x)$ to the end of the layer and sum them there, due to the interference effect between all these electric fields. In a continuous model this would mean integrating over the active layer thickness, in our Matlab implemented model this means we can just take a sum of discrete points:

$$
E_{\text {out }, \text { Total }}=\int_{0}^{L} E_{\text {out }}^{+}(x) d x \cong \sum_{\Delta x=n}^{\frac{L}{n}} E_{\text {out }}^{+}(x) \Delta x
$$

Where $E_{\text {out }}^{+}(x)$ the forwards-propagating plane wave originating from position $\mathrm{x}\left(\boldsymbol{E}_{*}^{+}(x)\right)$, but phase-shifted to the end of the layer by multiplying with $e^{\frac{2 \pi n}{\lambda}(L-x)} . \Delta x$, the step-size of the integral approximation, is set to $1 \mathrm{~nm}$ (as it has been previously for the transfer matrix implementations).

So now the total emission for a certain layer thickness can in principle be computed. However, there are still some important caveats to note:

1. There is also emitted light transmitted into the glass and reflected from the glass/air boundary. This light is incoherent with the original light and has to be treated separately. When calculating the contributions of this emission it turns out they are negligible. This is because of the effect of reabsorption on an already low fraction of light having to traverse the whole layer (see figure 5.9). 


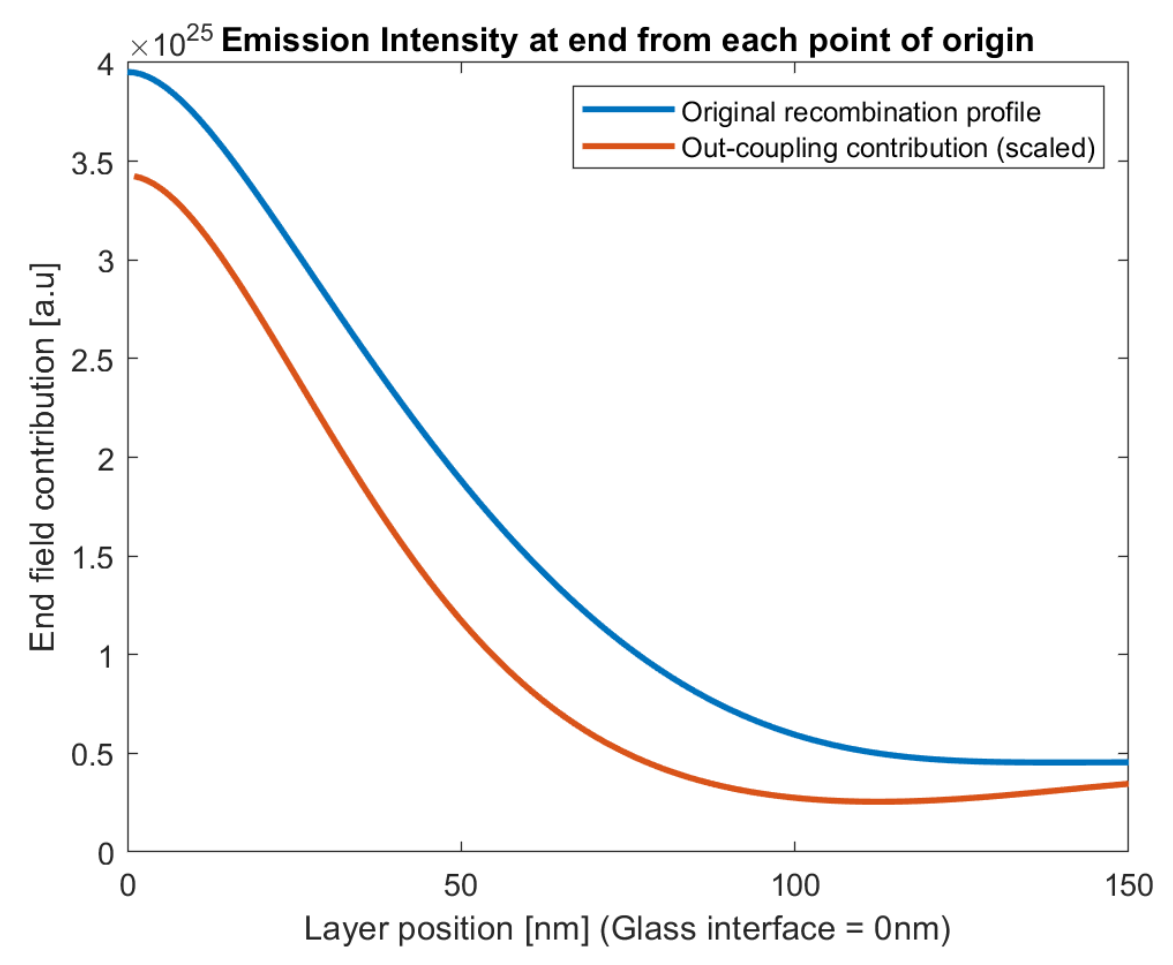

Figure 5.9. Layer Position Impact On Emission

Figure 5.9. A visual representation comparing the intensity of the original recombination profile to the intensity of the plane wave reaching the end of the layer from a certain position in the layer, adjusted for reabsorption and interference.

2. The light is actually measured at a non-normal angle, not perpendicular to the layer. This has two effects on the model, one is that the effective thickness of the layer is lengthened by $\frac{1}{\cos (\theta)}, \theta$ being the angle of incidence, and the second that the Fresnel coefficients need to be modified with the angle of incidence as well:

$$
\begin{aligned}
& r_{i j, s}=\frac{\bar{n}_{i} \cos \left(\theta_{i}\right)-\bar{n}_{j} \cos \left(\theta_{j}\right)}{\bar{n}_{i} \cos \left(\theta_{i}\right)+\bar{n}_{j} \cos \left(\theta_{j}\right)^{\prime}} \\
& r_{i j, p}=\frac{\bar{n}_{j} \cos \left(\theta_{i}\right)-\bar{n}_{i} \cos \left(\theta_{j}\right)}{\bar{n}_{j} \cos \left(\theta_{i}\right)+\bar{n}_{i} \cos \left(\theta_{j}\right)}
\end{aligned}
$$

This effectively produces two different contributions to the emission, one for the spolarized fraction of the emitted light and one for the p-polarized fraction (both fractions are equal since emitted light is isotropic).

With all this incorporated into the model, the out-coupling model is complete and we can move on to comparing to the Green's function model in Setfos, and finally to experimental data of emission measurements. 


\subsection{Experimental results}

Seven PCDTBT samples of varying thickness $(249 \mathrm{~nm}, 190 \mathrm{~nm}, 147 \mathrm{~nm}, 123 \mathrm{~nm}, 94 \mathrm{~nm}, 70 \mathrm{~nm}$, $31 \mathrm{~nm}$ ) were fabricated in a cleanroom glovebox $\left(<1 \mathrm{ppm} \mathrm{H}_{2} \mathrm{O}\right.$ and $\left.\mathrm{O}_{2}\right)$, all PCDTBT on glass. The films were spun cast from solution in Chlorobenzene (CB), with varying concentrations, and thicknesses were verified using both ellipsometry and the transmittance method. The emission of these samples was measured under a range of angles $\left(8^{\circ}-42^{\circ}\right.$, in $5^{\circ}$ steps $)$ at an excitation wavelength of 530nm. For this, an Oxxius laser was used, and the detection was implemented with a PMA spectral photon count detector (Figure 5.1). Then the same data was taken for a range of PCE10 samples on glass for a similar thickness range. Data of both measurement sets are shown in Figures 5.10 and 5.11.

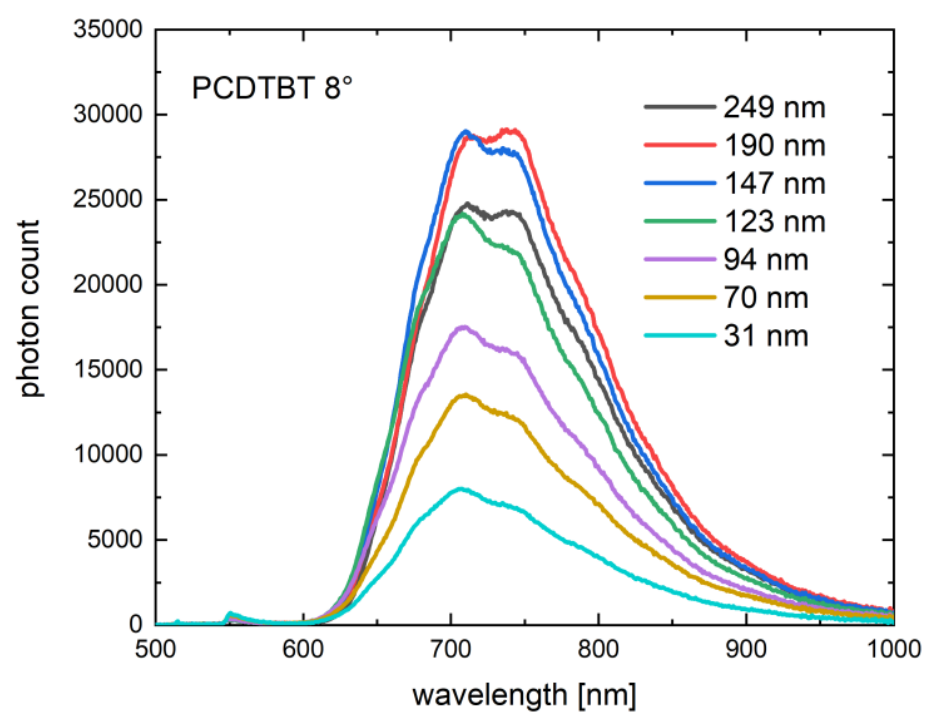

Figure 5.10. Spectral Emission Data PCDTBT

Figure 5.10. Spectral emission data for PCDTBT on glass, various layer thicknesses (indicated in the legend), taken with the rotating set-up shown in Figure 5.1. Standard angle of measurement was $8^{\circ}$. The sample was irradiated with an Oxxius laser at a wavelength of $532 \mathrm{~nm}$, and measured intensity was filtered out below 550nm. 


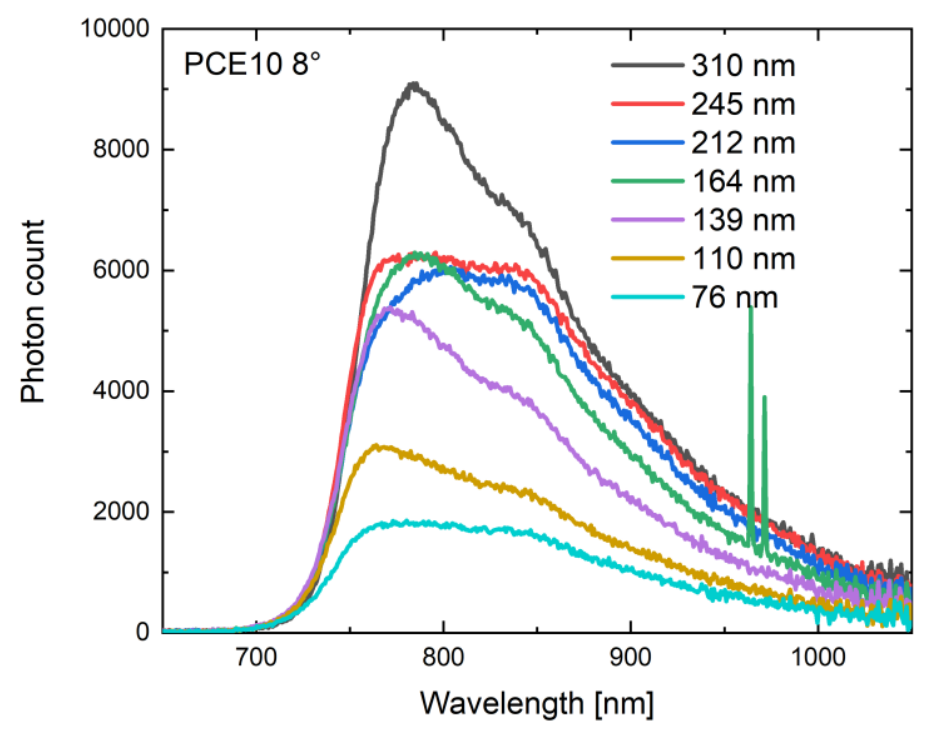

Figure 5.11. Spectral Emission Data PCE10

Figure 5.11. Spectral emission data for PCE10 on glass, various layer thicknesses, taken with the rotating set-up shown in Figure 5.1. Standard angle of measurement was $8^{\circ}$. The sample was irradiated with an Oxxius laser at a wavelength of $532 \mathrm{~nm}$, and measured intensity was filtered out below $550 \mathrm{~nm}$. A separate measurement of emission versus angle for a single film thickness was also undertaken as a secondary source of data (Figure 5.11). The integrated and peak intensity follow the expected solid angle cosine law.

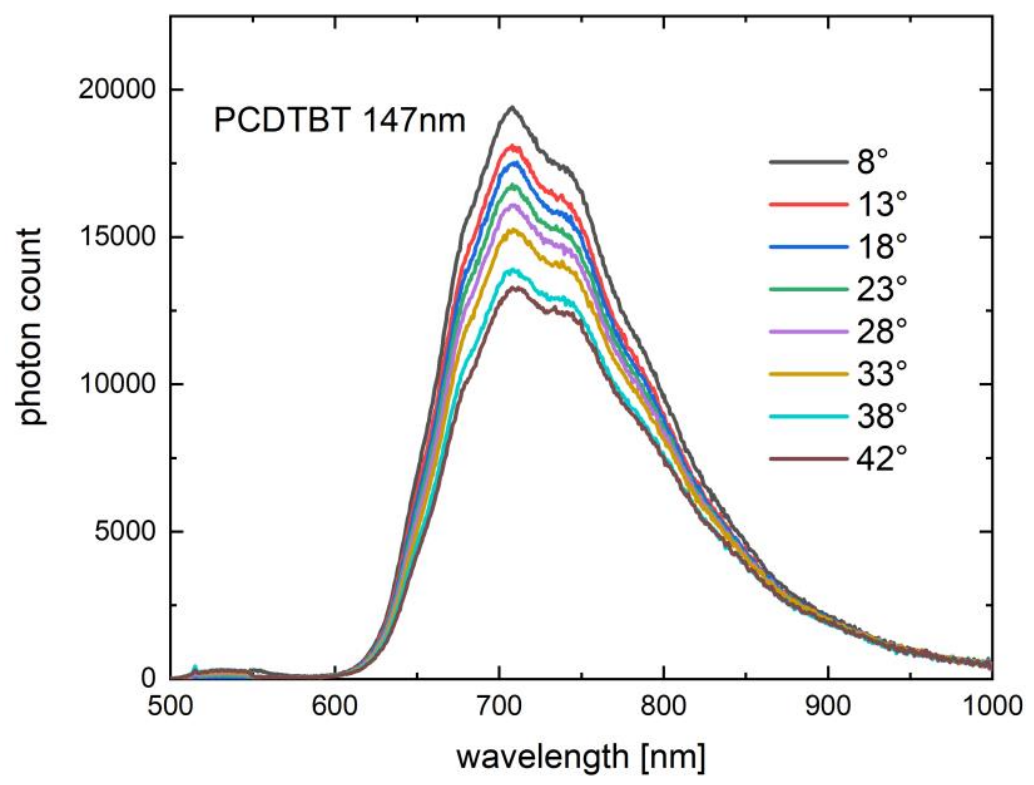

Figure 5.12. Angular Spectral Emission Data PCDTBT

Figure 5.12. Spectral emission data for PCDTBT on glass, various measurement angles, observed with the rotating set-up. Taken for a thickness of $147 \mathrm{~nm}$. 
Next, the model was tested as a predictor for the trend in relative emission due to interference effects when varying thickness. Since we are looking at a trend in relative emission, the data was scaled by a constant factor for direct comparison. Setfos emission predictions for the same sample thicknesses were also calculated and compared. The result of this comparison can be seen in figure 5.13 for PCDTBT and figure 5.14 for the PCE.

To check the reproducibility and accuracy of the measurements, a new set of samples over an extended thickness range was created for both materials, and measurements were taken multiple times to assess standard deviations (both Set 1 and Set2 are shown in the plot for comparison of the different sample batches). An important factor in obtaining reproducible data is sample quality, if the layer thickness is not uniform, emission data can vary quite significantly depending on where the sample is irradiated (as may be the case for samples like the $190 \mathrm{~nm}$ one where the standard deviation is high).

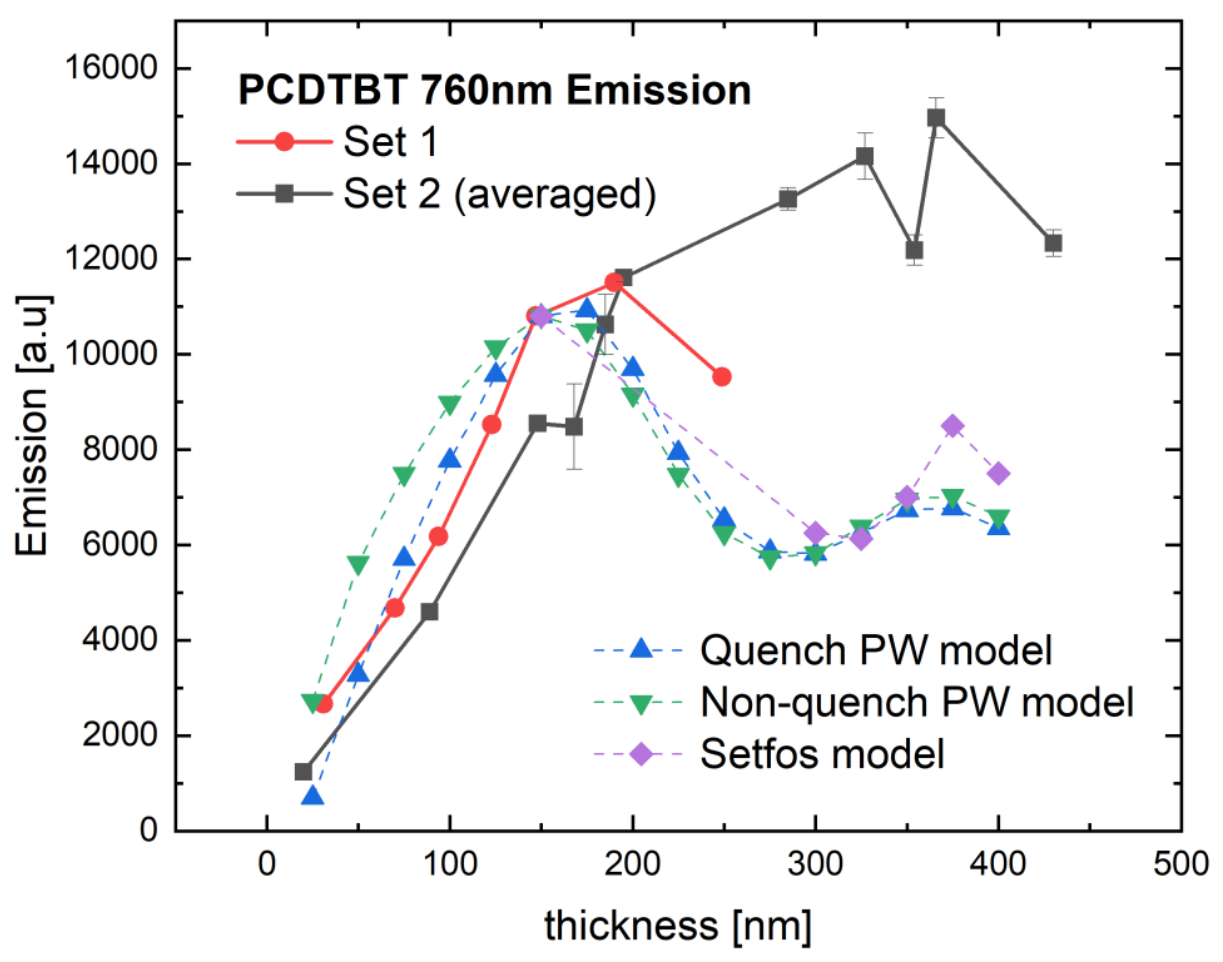

Figure 5.13. Emission Simulation and Data, PCDTBT

Figure 5.13. PCDTBT emission data for varying thicknesses, observed at a wavelength of $760 \mathrm{~nm}$. The Plane Wave out-coupling model is denoted by blue and green, where blue incorporates a quench approximation as outlined in section 5.5.2, and green is the plane wave model as is. 


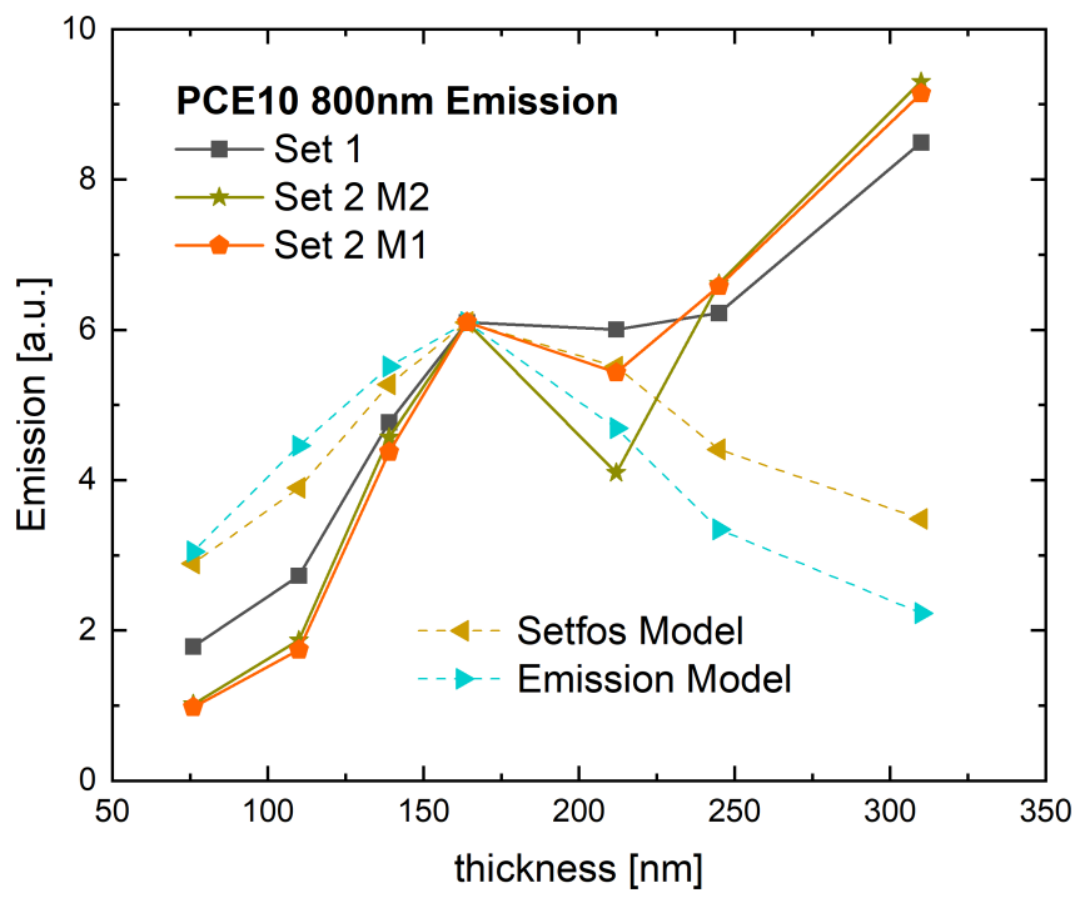

Figure 5.14. Emission Simulation and Data, PCE10

Figure 5.14. PCE10 emission data for varying thicknesses, observed at a wavelength of $800 \mathrm{~nm}$ (peak emission). Here only the quench approximation model is shown for the plane wave model since the difference between quench or no quench approximation is quite small.

The predictions are quite similar for both the Setfos and plane wave outcoupling models, and they agree with the trend in experimental data up to a thickness of around $200 \mathrm{~nm}$. After this the predictive abilities of the models break down. The reason for this is likely that both models fail to account for re-emission. As a result, they both underestimate the emission at thicker active layers. This omission is currently being addressed by the Setfos developers for a future upgrade.

Be that as it may, we can attempt to achieve our goal for a Marcus model fit free of interference effects, using the thicknesses for which our model appears to be valid, that is, up to $200 \mathrm{~nm}$. A comparison of the averaged original data (red) compared to the averaged interference corrected data (black), using 4 different sample thicknesses below $200 \mathrm{~nm}$, is shown in figure 5.15. As can be seen, the interference correction reduces the standard deviation at all wavelengths and gives an improved curve. The correction works by correcting the data by the factor of deviation that is predicted due to interference at that thickness (a simple relative scaling). Note that this will only be spectrally consistent if performed with a predictive model, not by an experimental baseline correction, which will vary spectrally. 


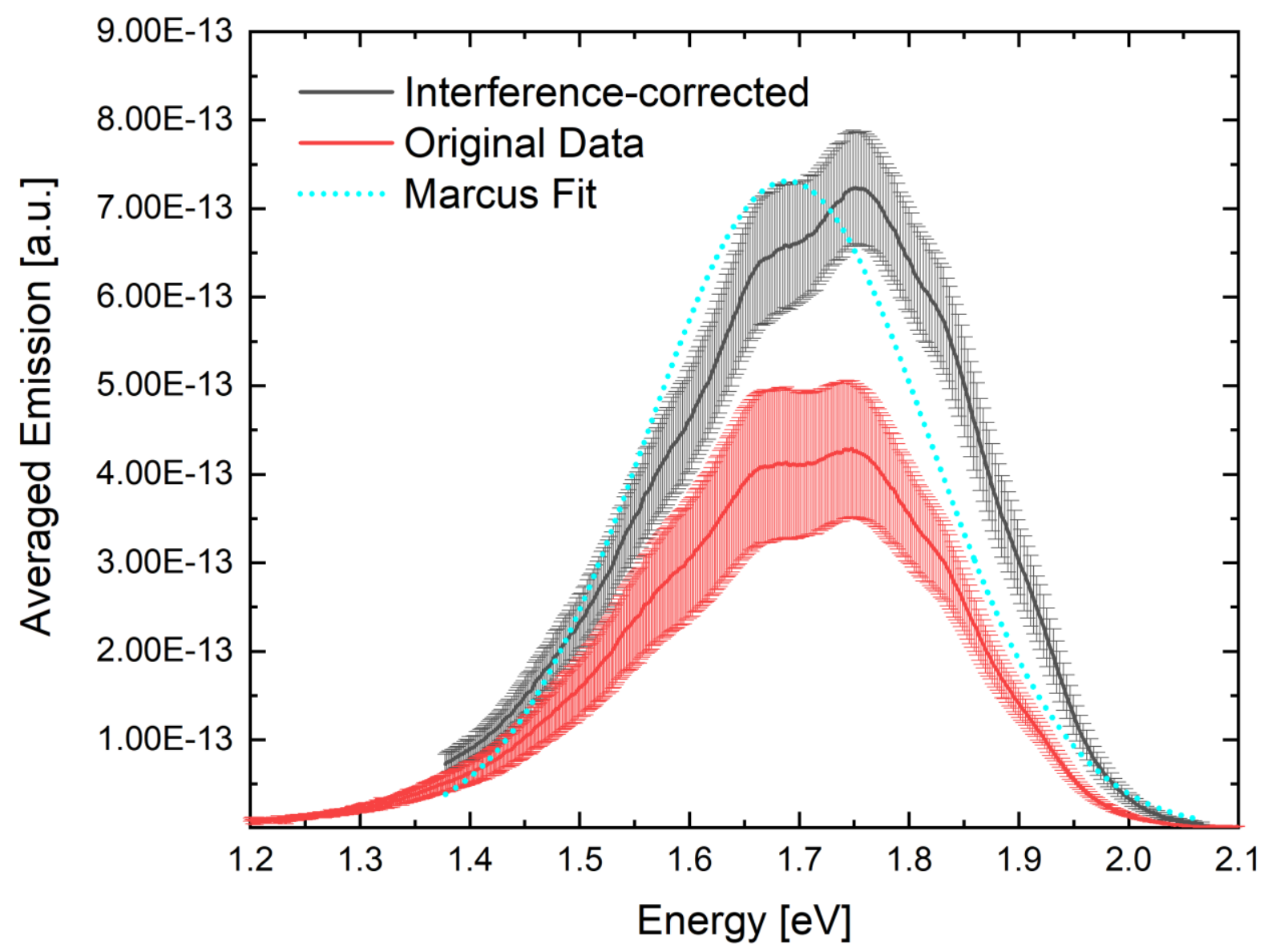

Figure 5.15. Averaged Emission Data Measured and Corrected.

Figure 5.15. Spectral emission data averaged over 4 PCDTBT samples of varying thickness. The red averaged data is not interference corrected and as such shows a large standard deviation from the average (which increases with more samples) and more uncertain peak. The black data is corrected using the PW emission model and gives a narrower deviation (which stays approximately the same with more samples added) and a more pronounced peak at $1.77 \mathrm{eV}$ $(700 \mathrm{~nm})$. A Marcus fit to the interference-corrected curve was also performed but does not converge since the equation, outlined in section 5.1, describes blends with a CT state and not neat materials like PCDTBT. However, the fit is demonstrative of the procedure that would be followed for a blend. Table $5 \mathrm{~A}$ below describes the fitting procedure in more detail. 
Table 5A. Details on the Marcus Fit of Averaged Emission in 5.15

\begin{tabular}{|l|c|}
\hline Model & $\begin{array}{c}\text { Ctpeak (User) } \\
\text { Equation }\end{array}$ \\
\hline $\begin{array}{c}\mathrm{x}^{*} \mathrm{ff} /\left(\text { (sqrt( }\left(4^{*} 3.14^{*} 0.026^{*} \text { lambda }\right)\right)^{*} \\
\text { exp(-(ect+lambda-x) } \\
\left.\left.\mathrm{a}^{*} 0.026\right)\right)\end{array}$ \\
\hline Plot & $\begin{array}{c}\text { Mean } \\
\text { lambda }\end{array}$ \\
\hline ect & $0.31903 \pm 0.00503$ \\
\hline ff & $1.36025 \pm 0.00583$ \\
\hline Reduced Chi-Sq & $1.40098 \mathrm{E}-13 \pm 1.98061 \mathrm{E}-15$ \\
\hline R-Square (COD & 3.35368 \\
\hline Adj. R-Square & 0.94046 \\
\hline
\end{tabular}

Table 5A. Converged parameters, in eV, for a Marcus fit to the interference-corrected curve from Figure 5.14, as outlined in section 5.1. The listed parameters are varied to achieve the best fit, where ect is the energy of the CT state $\left(E_{C T}\right)$, lambda is the reorganization energy $\left(\lambda_{C T}\right)$, and $f f$ the oscillator strength $\left(f_{\sigma}\right)$, as defined in Eq. 5.1b (repeated below for comparison). $x$ is the energy, which is given by the $\mathrm{x}$-axis of the figure. The equation is given in $\mathrm{eV}$ units.

$$
I=\frac{f_{I} E}{\sqrt{4 \pi \lambda_{\mathrm{CT}} k_{\mathrm{B}} T}} \exp \left(-\frac{\left(E_{\mathrm{CT}}+\lambda_{\mathrm{CT}}-E\right)^{2}}{4 \lambda_{\mathrm{CT}} k_{\mathrm{B}} T}\right)
$$

As mentioned before, these parameters are only defined for blends rather than neat materials, the fit is merely demonstrative in that sense. For blends, EL measurements would be more suitable. A suggestion of how the Plane Wave emission model could be extended in this direction is given in the next Chapter.

\subsection{Conclusion}

While the emission model would still need to be improved in regards with re-emission and EL to become truly useful, it does in its current form demonstrate how such a full model might be implemented. Additionally, we have shown that sample uniformity and measurement data quality are critical. As it is currently implemented, the model can already provide various valuable insights into the emission process, such as from where in the layer the majority of emitted light is coming from, how the recombination profile can be different from the generation profile due to diffusion of the excitons, and the importance of the thickness of the 
active layer material, which will give rise to significant, but predictable interference effects. In principle the model could eventually be extrapolated to blend materials, for which the Marcus fit was designed, but there are still various other effects that would have to be accounted for as well, such as the exciton dissociation into electrons and holes (which might affect the recombination profile significantly) and existence of charge transfer states. For blends it may indeed be argued that Electroluminescence measurements are better suited, because then the recombination profile will be a quasi-Dirac function where both carriers meet. However, the out-coupling model as presented here would remain virtually unchanged. 


\section{Conclusions and outlook}

This thesis describes a body of work focused on furthering our understanding of the optoelectrical physics of next generation semiconductor materials and devices - notably organic and organohalide perovskite semiconductors for photovoltaics and in a more limited sense light emission. The majority of the content focusses on the assessment and adaptation of optical-electrical methods and models used routinely in inorganic semiconductor systems: optical constant determination via ellipsometry and spectrophotometry; transfer matrix and finite element; thermodynamic reciprocity and the treatment of light emission. Using this suite of capabilities, several novel second and third generation photovoltaic systems have been examined, and in one rather extreme case - the perovskite carbon stack architecture quite significant new insight was gained, namely a thickness-dependent feature that allows devices with a non-selective contact to still attain viable efficiencies. Another important outcome of this work has been the additional information gained on the newly emergent nonfullerene electron acceptors which are proving very different to their second-generation fullerene counterparts.

A significant part of work presented in this thesis focused on the development of an alternative method for optical constant determination of high dielectric constant materials, highlighting the benefits of a transmittance method in supplement to conventional ellipsometry. This method was made use of repetitively for characterizing challenging organic and organohalide perovskite semiconductors and has significant resource advantages over ellipsometry. The method was fully validated using a number of very challenging systems. It will likely continue to be helpful to the next generation semiconductor and opto-electrical and materials communities and we have made freeware code available for anyone to use $\mathrm{e}^{103}$. Another important outcome of this element of the work has been the additional information gained on the newly emergent non-fullerene electron acceptors which are proving very different to their second-generation fullerene counterparts. Notably, they have significantly higher optical constants and complementary absorption when in an acceptor: donor blend. Both of these characteristics have significant impacts of device-level opto-electrical properties and notably low finesse cavity effects. 
In relation to the carbon contact perovskite solar cell (perovskite carbon stack), the structure benefits from comparatively thick, micron-scale active layers, which are not only attractive from a manufacturing perspective, but also enable a cheap and electrically non-selective mesoporous carbon anode to be utilised. The key opto-electrical finding was that the nonselective contact works because of the imbalance between minority and majority carriers at that electrode resulting in relatively low levels of contact-related recombination. This architecture is gaining considerable interest for manufacturing and scaling, and improvements are also being investigated including using more stable forms of perovskite such as the triple cation blend ${ }^{101}$, attempting to improve the perovskite infiltration by adding various wetting agents ${ }^{102}$, and improving the contact in ways that maintain the industrial fabrication process, such as the addition of Nickel Oxide particles to the mesoporous carbon paste $^{70}$.

Another significant device structure that is still currently being developed and pursued vigorously is the perovskite-silicon tandem solar stack. An opto-electrical model including thick incoherent layers was developed and presented. This model can now be used to optimize specific implementations of the tandem stack, to derive the parameters for highest possible efficiencies (such as spacer and transport layers, perovskite bandgap and layer thickness, etc.) before fabrication. An additional electric charge transfer model, utilizing knowledge of interfacial recombination rates of various interlayers and layer mobilities, could be added to this optimization model in the future. That would be a valuable contribution as next steps.

Core to the modelling-centric work described in this thesis was the use of the transfer matrix method, which was described in detail theoretically and implemented in all previously mentioned endeavours. The theory behind this method, accounting for various interference effects in multi-layer device stacks, was used as the basis to derive a new type of model that accounts for interference effects in photo-emission measurements. Cavity effects in emission are significant in devices like thin film LEDs and EL measurements not properly correct for interference are often used in combination with reciprocity to establish CT state parameters. Our so-called plane wave model was able to successfully model, reconstruct and correct photoluminescence and decouple PL measurements from layer thickness interference and 
was validated versus a Setfos finite element calculation. A script implementing this new model is also available as open-source ${ }^{103}$.

Current limitations for the plane wave model include the modelling of re-emission, which requires measurement of another free variable: the probability of radiative recombination $P_{r}$. For this the rates of radiative recombination and of non-radiative recombination need to be known (see Eq. 5.6). In future work, these might be determined experimentally and incorporated into the model. Additionally, the plane wave model would only truly become useful for organic semiconductor blend materials, which tend to have lower photoluminescence. For this reason, electroluminescence would be the target measurement. In principle, electro-luminescence changes nothing about the plane wave model, with the exception that the recombination profile is no longer a diffusion-shifted generation profile but becomes a quasi-Dirac peak function at the location in the layer where injected charge carriers meet. Thus, the model should be able to be extended towards organic semiconductor blend materials and provide valuable insights on charge transfer state properties, which is a key property of organic blends still under much discussion today 95, 96, 104, 105.

In summary, the research presented in this thesis has further advanced our understanding of the opto-electrical physics of next generation semiconductors and related light harvesting and emissive devices. New analytical approaches have been developed and made available to the community and insight gained into several state-of-the-art device architectures such as the perovskite carbon stack, non-fullerene based organic solar cells and perovskite-silicon tandems. Finally, a window has been opened on the treatment of emissive junctions to account for cavity interference effects. This once again highlights the old adage that a 'good solar cell is a good light emitting diode' and the long-held principles of thermodynamic reciprocity at the heart of our field. 


\section{Bibliography}

1. Wöhrle D, Meissner D. Organic solar cells. Advanced Materials 1991, 3:129-138.

2. Günes S, Neugebauer H, Sariciftci NS. Conjugated polymer-based organic solar cells. Chemical reviews 2007, 107:1324-1338.

3. Yamashita Y. Organic semiconductors for organic field-effect transistors. Science and technology of advanced materials 2009.

4. Xue J. Perspectives on organic photovoltaics. Polymer Reviews 2010, 50:411-419.

5. Yamasaki A. An overview of $\mathrm{CO} 2$ mitigation options for global warming-emphasizing $\mathrm{CO} 2$ sequestration options. Journal of chemical engineering of Japan 2003, 36:361-375.

6. Misra A, Kumar P, Kamalasanan M, Chandra S. White organic LEDs and their recent advancements. Semiconductor science and Technology 2006, 21:R35.

7. Horowitz G. Organic field-effect transistors. Advanced materials 1998, 10:365-377.

8. Spear W, Le Comber P. Substitutional doping of amorphous silicon. Solid state communications 1975, 17:1193-1196.

9. Knechtli RC, Loo RY, Kamath GS. High-efficiency GaAs solar cells. IEEE Transactions on electron devices 1984, 31:577-588.

10. Jester TL. Crystalline silicon manufacturing progress. Progress in Photovoltaics: Research and Applications 2002, 10:99-106.

11. Masuko K, Shigematsu M, Hashiguchi T, Fujishima D, Kai M, Yoshimura N, Yamaguchi T, Ichihashi Y, Mishima T, Matsubara N. Achievement of more than $25 \%$ conversion efficiency with crystalline silicon heterojunction solar cell. IEEE Journal of Photovoltaics 2014, 4:14331435.

12. Uno M, Nakayama K, Soeda J, Hirose Y, Miwa K, Uemura T, Nakao A, Takimiya K, Takeya J. High-Speed Flexible Organic Field-Effect Transistors with a 3D Structure. Advanced Materials 2011, 23:3047-3051.

13. Mizukami M, Cho S-I, Watanabe K, Abiko M, Suzuri Y, Tokito S, Kido J. Flexible organic lightemitting diode displays driven by inkjet-printed high-mobility organic thin-film transistors. IEEE Electron Device Letters 2017, 39:39-42.

14. Schwab K. The fourth industrial revolution: Currency; 2017.

15. Schubert MB, Werner JH. Flexible solar cells for clothing. Materials today 2006, 9:42-50.

16. Torsi L, Magliulo M, Manoli K, Palazzo G. Organic field-effect transistor sensors: a tutorial review. Chemical Society Reviews 2013, 42:8612-8628.

17. Miyazaki T, Akisawa A, Kashiwagi T. Energy savings of office buildings by the use of semitransparent solar cells for windows. Renewable energy 2005, 30:281-304.

18. Kittel C. Introduction to solid state physics. 1976.

19. Pivrikas A, Sariciftci NS, Juška G, Österbacka R. A review of charge transport and recombination in polymer/fullerene organic solar cells. Progress in Photovoltaics: Research and Applications 2007, 15:677-696.

20. Stranks SD, Burlakov VM, Leijtens T, Ball JM, Goriely A, Snaith HJ. Recombination kinetics in organic-inorganic perovskites: excitons, free charge, and subgap states. Physical Review Applied 2014, 2:034007.

21. Baranovskii S, Wiemer M, Nenashev A, Jansson F, Gebhard F. Calculating the efficiency of exciton dissociation at the interface between a conjugated polymer and an electron acceptor. The Journal of Physical Chemistry Letters 2012, 3:1214-1221.

22. Lin Q, Armin A, Nagiri RCR, Burn PL, Meredith P. Electro-optics of perovskite solar cells. Nature Photonics 2015, 9:106-112.

23. Yan J, Saunders BR. Third-generation solar cells: a review and comparison of polymer: fullerene, hybrid polymer and perovskite solar cells. Rsc Advances 2014, 4:43286-43314. 
24. Ono S, Ohno K. Combined impact of entropy and carrier delocalization on charge transfer exciton dissociation at the donor-acceptor interface. Physical Review B 2016, 94:075305.

25. Saliba M, Matsui T, Seo J-Y, Domanski K, Correa-Baena J-P, Nazeeruddin MK, Zakeeruddin SM, Tress W, Abate A, Hagfeldt A. Cesium-containing triple cation perovskite solar cells: improved stability, reproducibility and high efficiency. Energy \& Environmental Science 2016, 9:1989-1997.

26. Niu G, Guo X, Wang L. Review of recent progress in chemical stability of perovskite solar cells. Journal of Materials Chemistry A 2015, 3:8970-8980.

27. Zhang J, Bai D, Jin Z, Bian H, Wang K, Sun J, Wang Q, Liu S. 3D-2D-0D interface profiling for record efficiency all-inorganic $\mathrm{CsPbBr} 2$ Perovskite solar cells with superior stability. Advanced Energy Materials 2018, 8:1703246.

28. Seo JY, Uchida R, Kim HS, Saygili Y, Luo J, Moore C, Kerrod J, Wagstaff A, Eklund M, McIntyre $\mathrm{R}$. Boosting the efficiency of perovskite solar cells with $\mathrm{CsBr}$-modified mesoporous TiO2 beads as electron-selective contact. Advanced Functional Materials 2018, 28:1705763.

29. Baker J, Hooper K, Meroni S, Pockett A, McGettrick J, Wei Z, Escalante R, Oskam G, Carnie M, Watson T. High throughput fabrication of mesoporous carbon perovskite solar cells. Journal of Materials Chemistry A 2017, 5:18643-18650.

30. Heumueller T, Mateker WR, Sachs-Quintana I, Vandewal K, Bartelt JA, Burke TM, Ameri T, Brabec CJ, McGehee MD. Reducing burn-in voltage loss in polymer solar cells by increasing the polymer crystallinity. Energy \& Environmental Science 2014, 7:2974-2980.

31. Zhang Y, Liu M, Eperon GE, Leijtens TC, McMeekin D, Saliba M, Zhang W, de Bastiani M, Petrozza A, Herz LM. Charge selective contacts, mobile ions and anomalous hysteresis in organic-inorganic perovskite solar cells. Materials Horizons 2015, 2:315-322.

32. Zhao C, Chen B, Qiao X, Luan L, Lu K, Hu B. Revealing underlying processes involved in light soaking effects and hysteresis phenomena in perovskite solar cells. Advanced Energy Materials 2015, 5:1500279.

33. Jha A, Duan H-G, Tiwari V, Nayak PK, Snaith HJ, Thorwart M, Miller RD. Direct observation of ultrafast exciton dissociation in lead iodide perovskite by 2D electronic spectroscopy. ACS Photonics 2017, 5:852-860.

34. Wolff CM, Caprioglio P, Stolterfoht $\mathrm{M}$, Neher D. Nonradiative recombination in perovskite solar cells: the role of interfaces. Advanced Materials 2019, 31:1902762.

35. Yan C, Barlow S, Wang Z, Yan H, Jen AK-Y, Marder SR, Zhan X. Non-fullerene acceptors for organic solar cells. Nature Reviews Materials 2018, 3:1-19.

36. Liu J, Chen S, Qian D, Gautam B, Yang G, Zhao J, Bergqvist J, Zhang F, Ma W, Ade H. Fast charge separation in a non-fullerene organic solar cell with a small driving force. Nature Energy 2016, 1:1-7.

37. Kotova MS, Londi G, Junker J, Dietz S, Privitera A, Tvingstedt K, Beljonne D, Sperlich A, Dyakonov $\mathrm{V}$. On the absence of triplet exciton loss pathways in non-fullerene acceptor based organic solar cells. Materials Horizons 2020, 7:1641-1649.

38. Pan M-A, Lau T-K, Tang Y, Wu Y-C, Liu T, Li K, Chen M-C, Lu X, Ma W, Zhan C. 16.7\%-efficiency ternary blended organic photovoltaic cells with PCBM as the acceptor additive to increase the open-circuit voltage and phase purity. Journal of Materials Chemistry A 2019, 7:2071320722.

39. Bridgman P. Note on the principle of detailed balancing. Physical Review 1928, 31:101.

40. Würfel P, Würfel U. Physics of solar cells: from basic principles to advanced concepts: John Wiley \& Sons; 2016.

41. Shockley W, Queisser HJ. Detailed balance limit of efficiency of p-n junction solar cells. Journal of applied physics 1961, 32:510-519.

42. Yoshikawa K, Kawasaki H, Yoshida W, Irie T, Konishi K, Nakano K, Uto T, Adachi D, Kanematsu $\mathrm{M}, \mathrm{Uzu} \mathrm{H}$. Silicon heterojunction solar cell with interdigitated back contacts for a photoconversion efficiency over $26 \%$. Nature energy 2017, 2:1-8. 
43. Armin A, Velusamy M, Wolfer P, Zhang Y, Burn PL, Meredith P, Pivrikas A. Quantum efficiency of organic solar cells: electro-optical cavity considerations. Acs Photonics 2014, 1:173-181.

44. Lax M. The Franck-Condon principle and its application to crystals. The Journal of chemical physics 1952, 20:1752-1760.

45. Kirchartz T. Generalized detailed balance theory of solar cells. Vol. 38: Forschungszentrum Jülich; 2009.

46. Yang J-P, Bussolotti F, Kera S, Ueno N. Origin and role of gap states in organic semiconductor studied by UPS: as the nature of organic molecular crystals. Journal of Physics D: Applied Physics 2017, 50:423002.

47. Harbecke B. Coherent and incoherent reflection and transmission of multilayer structures. Applied Physics B 1986, 39:165-170.

48. Pettersson LA, Roman LS, Inganäs O. Modeling photocurrent action spectra of photovoltaic devices based on organic thin films. Journal of Applied Physics 1999, 86:487-496.

49. Jin H, Tao C, Velusamy M, Aljada M, Zhang Y, Hambsch M, Burn PL, Meredith P. Efficient, large area ITO-and-PEDOT-free organic solar cell sub-modules. Advanced materials 2012, 24:2572-2577.

50. Chance R, Prock A, Silbey R. Molecular fluorescence and energy transfer near interfaces. Adv. Chem. Phys 1978, 37:1-65.

51. Berger P, Kim M. Polymer solar cells: P3HT: PCBM and beyond. Journal of Renewable and Sustainable Energy 2018, 10:013508.

52. Ke Y, Liu Y, Zhao Y. Visualization of hot exciton energy relaxation from coherent to diffusive regimes in conjugated polymers: a theoretical analysis. The journal of physical chemistry letters 2015, 6:1741-1747.

53. Gurney RS, Lidzey DG, Wang T. A review of non-fullerene polymer solar cells: from device physics to morphology control. Reports on Progress in Physics 2019, 82:036601.

54. Nan G, Zhang X, Lu G. Do "Hot" Charge-Transfer Excitons Promote Free Carrier Generation in Organic Photovoltaics? The Journal of Physical Chemistry C 2015, 119:15028-15035.

55. Stübinger T, Brütting W. Exciton diffusion and optical interference in organic donor-acceptor photovoltaic cells. Journal of Applied Physics 2001, 90:3632-3641.

56. Kahle F-J, Rudnick A, Bässler H, Köhler A. How to interpret absorption and fluorescence spectra of charge transfer states in an organic solar cell. Materials Horizons 2018, 5:837-848.

57. List M, Sarkar T, Perkhun P, Ackermann J, Luo C, Würfel U. Correct determination of charge transfer state energy from luminescence spectra in organic solar cells. Nature communications 2018, 9:1-8.

58. Kerremans R, Kaiser C, Li W, Zarrabi N, Meredith P, Armin A. The Optical Constants of Solution-Processed Semiconductors - New Challenges with Perovskites and Non-Fullerene Acceptors. Advanced Optical Materials 2020, 8:2000319.

59. Stenner MD, Gauthier DJ, Neifeld MA. The speed of information in a 'fast-light'optical medium. Nature 2003, 425:695-698.

60. Heavens OS. Optical properties of thin solid films: Courier Corporation; 1991.

61. Pettersson LAA, Roman LS, Inganäs $O$. Modeling photocurrent action spectra of photovoltaic devices based on organic thin films. Journal of Applied Physics 1999, 86:487-496.

62. Woollam JA, Johs BD, Herzinger CM, Hilfiker JN, Synowicki RA, Bungay CL. Overview of variable-angle spectroscopic ellipsometry (VASE): I. Basic theory and typical applications. In: Optical Metrology: A Critical Review: International Society for Optics and Photonics; 1999.

63. Li X, Tschumi M, Han H, Babkair SS, Alzubaydi RA, Ansari AA, Habib SS, Nazeeruddin MK, Zakeeruddin SM, Grätzel M. Outdoor performance and stability under elevated temperatures and long-term light soaking of triple-layer mesoporous perovskite photovoltaics. Energy Technology 2015, 3:551-555. 
64. Zhao Y, Nardes AM, Zhu K. Mesoporous perovskite solar cells: material composition, chargecarrier dynamics, and device characteristics. Faraday discussions 2015, 176:301-312.

65. Hagfeldt A, Boschloo G, Sun L, Kloo L, Pettersson H. Dye-sensitized solar cells. Chemical reviews 2010, 110:6595-6663.

66. Kochergin V, Christophersen M, Föll H. Effective medium approach for calculations of optical anisotropy in porous materials. Applied Physics B 2004, 79:731-739.

67. Poelman D, Smet PF. Methods for the determination of the optical constants of thin films from single transmission measurements: a critical review. Journal of Physics D: Applied Physics 2003, 36:1850.

68. Etzold F, Howard IA, Mauer R, Meister M, Kim T-D, Lee K-S, Baek NS, Laquai F. Ultrafast exciton dissociation followed by nongeminate charge recombination in PCDTBT: PCBM photovoltaic blends. Journal of the American Chemical Society 2011, 133:9469-9479.

69. Kerremans R, Sandberg OJ, Meroni S, Watson T, Armin A, Meredith P. On the Electro-Optics of Carbon Stack Perovskite Solar Cells. Solar RRL 2019:1900221.

70. Lakhiani H, Dunlop T, De Rossi F, Dimitrov S, Kerremans R, Charbonneau C, Watson T, Barbé J, Tsoi WC. Variations of Infiltration and Electronic Contact in Mesoscopic Perovskite Solar Cells Revealed by High-Resolution Multi-Mapping Techniques. Advanced Functional Materials 2019, 29:1900885.

71. Zhang Z-G, Qi B, Jin Z, Chi D, Qi Z, Li Y, Wang J. Perylene diimides: a thickness-insensitive cathode interlayer for high performance polymer solar cells. Energy \& Environmental Science 2014, 7:1966-1973.

72. Kim YH, Sachse C, Machala ML, May C, Müller-Meskamp L, Leo K. Highly conductive PEDOT: PSS electrode with optimized solvent and thermal post-treatment for ITO-free organic solar cells. Advanced Functional Materials 2011, 21:1076-1081.

73. Ossila. Available at: https://www.ossila.com/products/pv-substrates?variant=1200245025.

74. Ohta $\mathrm{K}$, Ishida $\mathrm{H}$. Matrix formalism for calculation of electric field intensity of light in stratified multilayered films. Applied optics 1990, 29:1952-1959.

75. Mei A, Li X, Liu L, Ku Z, Liu T, Rong Y, Xu M, Hu M, Chen J, Yang Y. A hole-conductor-free, fully printable mesoscopic perovskite solar cell with high stability. Science 2014, 345:295298.

76. Shao L-L, Chen M, Ren T-Z, Yuan Z-Y. Ordered mesoporous carbon/graphene nano-sheets composites as counter electrodes in dye-sensitized solar cells. Journal of Power Sources 2015, 274:791-798.

77. Lee HKH, Barbé J, Meroni SM, Du T, Lin CT, Pockett A, Troughton J, Jain SM, De Rossi F, Baker J. Outstanding Indoor Performance of Perovskite Photovoltaic Cells-Effect of Device Architectures and Interlayers. Solar RRL 2019, 3:1800207.

78. Meroni SM, Mouhamad Y, De Rossi F, Pockett A, Baker J, Escalante R, Searle J, Carnie MJ, Jewell $\mathrm{E}$, Oskam G. Homogeneous and highly controlled deposition of low viscosity inks and application on fully printable perovskite solar cells. Science and Technology of Advanced Materials 2018, 19:1-9.

79. De Rossi F, Baker JA, Beynon D, Hooper KE, Meroni SM, Williams D, Wei Z, Yasin A, Charbonneau C, Jewell EH. All printable perovskite solar modules with $198 \mathrm{~cm} 2$ active area and over 6\% efficiency. Advanced Materials Technologies 2018, 3:1800156.

80. Herz LM. Charge-carrier mobilities in metal halide perovskites: fundamental mechanisms and limits. ACS Energy Letters 2017, 2:1539-1548.

81. Lin Q, Armin A, Nagiri RCR, Burn PL, Meredith P. Electro-optics of perovskite solar cells. Nature Photonics 2014, 9:106-112.

82. Asensi J, Merten J, Voz C, Andreu J. Analysis of the role of mobility-lifetime products in the performance of amorphous silicon pin solar cells. Journal of Applied Physics 1999, 85:29392951. 
83. Li Z, Chiu KH, Ashraf RS, Fearn S, Dattani R, Wong HC, Tan C-H, Wu J, Cabral JT, Durrant JR. Toward improved lifetimes of organic solar cells under thermal stress: substrate-dependent morphological stability of PCDTBT: PCBM films and devices. Scientific reports 2015, 5:1-9.

84. Staniec PA, Parnell AJ, Dunbar AD, Yi H, Pearson AJ, Wang T, Hopkinson PE, Kinane C, Dalgliesh RM, Donald AM. The nanoscale morphology of a PCDTBT: PCBM photovoltaic blend. Advanced Energy Materials 2011, 1:499-504.

85. Clarke TM, Peet J, Nattestad A, Drolet N, Dennler G, Lungenschmied C, Leclerc M, Mozer AJ. Charge carrier mobility, bimolecular recombination and trapping in polycarbazole copolymer: fullerene (PCDTBT: PCBM) bulk-heterojunction solar cells. Organic Electronics 2012, 13:2639-2646.

86. Zhang S, Stolterfoht M, Armin A, Lin Q, Zu F, Sobus J, Jin H, Koch N, Meredith P, Burn PL. Interface Engineering of Solution-Processed Hybrid Organohalide Perovskite Solar Cells. ACS applied materials \& interfaces 2018, 10:21681-21687.

87. Aspnes D. Local-field effects and effective-medium theory: a microscopic perspective. American Journal of Physics 1982, 50:704-709.

88. Al-Ashouri A, Köhnen E, Li B, Magomedov A, Hempel H, Caprioglio P, Márquez JA, Vilches ABM, Kasparavicius E, Smith JA. Monolithic perovskite/silicon tandem solar cell with $>29 \%$ efficiency by enhanced hole extraction. Science 2020, 370:1300-1309.

89. Duong T, Wu Y, Shen H, Peng J, Fu X, Jacobs D, Wang EC, Kho TC, Fong KC, Stocks M. Rubidium multication perovskite with optimized bandgap for perovskite-silicon tandem with over 26\% efficiency. Advanced Energy Materials 2017, 7:1700228.

90. Saliba M, Correa-Baena J-P, Wolff CM, Stolterfoht M, Phung N, Albrecht S, Neher D, Abate A. How to Make over 20\% Efficient Perovskite Solar Cells in Regular ( $n-i-p)$ and Inverted ( $p-i-$ n) Architectures. Chemistry of Materials 2018, 30:4193-4201.

91. Perdigón-Toro L, Zhang H, Markina A, Yuan J, Hosseini SM, Wolff CM, Zuo G, Stolterfoht M, Zou Y, Gao F. Barrierless free charge generation in the high-performance PM6: Y6 bulkheterojunction non-fullerene solar cell. Advanced Materials 2020, 32:1906763.

92. Zhan L, Li S, Lau T-K, Cui Y, Lu X, Shi M, Li C-Z, Li H, Hou J, Chen H. Over $17 \%$ efficiency ternary organic solar cells enabled by two non-fullerene acceptors working in an alloy-like model. Energy \& Environmental Science 2020, 13:635-645.

93. Trupke T, Bardos R, Hudert F, Würfel P, Zhao J, Wang A, Green M. Effective excess carrier lifetimes exceeding 100 milliseconds in float zone silicon determined from photoluminescence. In: Proceedings of the 19th European Photovoltaic Solar Energy Conference, Paris, France; 2004.

94. Zhu L, Zhang M, Zhou G, Hao T, Xu J, Wang J, Qiu C, Prine N, Ali J, Feng W. Efficient organic solar cell with $16.88 \%$ efficiency enabled by refined acceptor crystallization and morphology with improved charge transfer and transport properties. Advanced Energy Materials 2020, 10:1904234.

95. Meredith P, Li W, Armin A. Nonfullerene Acceptors: a renaissance in organic photovoltaics? Advanced Energy Materials 2020, 10:2001788.

96. Armin A, Zarrabi N, Sandberg OJ, Kaiser C, Zeiske S, Li W, Meredith P. Limitations of Charge Transfer State Parameterization Using Photovoltaic External Quantum Efficiency. Advanced Energy Materials 2020, 10:2001828.

97. Chauhan A, Gupta S, Taguchi D, Manaka T, Jha P, Veerender P, Sridevi C, Koiry S, Gadkari S, Iwamoto $M$. Enhancement of the carrier mobility of conducting polymers by formation of their graphene composites. RSC advances 2017, 7:11913-11920.

98. Kim T, Yang SJ, Kim SK, Choi HS, Park CR. Preparation of PCDTBT nanofibers with a diameter of $20 \mathrm{~nm}$ and their application to air-processed organic solar cells. Nanoscale 2014, 6:28472854. 
99. Chutinan A, Ishihara K, Asano T, Fujita M, Noda S. Theoretical analysis on light-extraction efficiency of organic light-emitting diodes using FDTD and mode-expansion methods. Organic electronics 2005, 6:3-9.

100. Stokes shift image. Available at: https://chemistry.stackexchange.com/questions/55574/why-are-the-absorption-andemission-spectra-mirror-images-of-each-other.

101. Papadatos D, Sygkridou D, Stathatos E. Carbon-based, novel triple cation mesoscopic perovskite solar cell fabricated entirely under ambient air conditions. Materials Letters 2020, 268:127621.

102. Liu Z, Zhang M, Xu X, Bu L, Zhang W, Li W, Zhao Z, Wang M, Cheng Y-B, He H. p-Type mesoscopic $\mathrm{NiO}$ as an active interfacial layer for carbon counter electrode based perovskite solar cells. Dalton Transactions 2015, 44:3967-3973.

103. Kerremans R. RK Github. Available at: www.github.com/robinkerremans.

104. Karuthedath S, Gorenflot J, Firdaus Y, Chaturvedi N, De Castro CS, Harrison GT, Khan JI, Markina A, Balawi AH, Peña TAD. Intrinsic efficiency limits in low-bandgap non-fullerene acceptor organic solar cells. Nature Materials 2020:1-7.

105. Hodgkiss JM. No free lunch for non-fullerene acceptors. Nature Materials 2020:1-2.

106. Saliba M, Matsui T, Seo J-Y, Domanski K, Correa-Baena J-P, Nazeeruddin MK, Zakeeruddin SM, Tress W, Abate A, Hagfeldt A, et al. Cesium-containing triple cation perovskite solar cells: improved stability, reproducibility and high efficiency. Energy \& Environmental Science 2016, 9:1989-1997.

107. Li Z, Yang M, Park J-S, Wei S-H, Berry JJ, Zhu K. Stabilizing perovskite structures by tuning tolerance factor: formation of formamidinium and cesium lead iodide solid-state alloys. Chemistry of Materials 2016, 28:284-292.

108. Bishop JE, Read CD, Smith JA, Routledge TJ, Lidzey DG. Fully spray-coated triple-cation perovskite solar cells. Scientific reports 2020, 10:1-8.

109. Zheng J, Zhang M, Lau CFJ, Deng X, Kim J, Ma Q, Chen C, Green MA, Huang S, Ho-Baillie AW. Spin-coating free fabrication for highly efficient perovskite solar cells. Solar Energy Materials and Solar Cells 2017, 168:165-171.

110. Wu J, Xu X, Zhao Y, Shi J, Xu Y, Luo Y, Li D, Wu H, Meng Q. DMF as an additive in a two-step spin-coating method for $20 \%$ conversion efficiency in perovskite solar cells. ACS applied materials \& interfaces 2017, 9:26937-26947.

111. Grancini G, Roldán-Carmona C, Zimmermann I, Mosconi E, Lee X, Martineau D, Narbey S, Oswald F, De Angelis F, Graetzel M. One-Year stable perovskite solar cells by 2D/3D interface engineering. Nature communications 2017, 8:1-8.

112. Wang R, Xue J, Meng L, Lee J-W, Zhao Z, Sun P, Cai L, Huang T, Wang Z, Wang Z-K. Caffeine improves the performance and thermal stability of perovskite solar cells. Joule 2019, 3:14641477. 


\section{Appendix A1}

This appendix will briefly discuss incoherence in a multilayer stack and how to incorporate it into transfer matrix formalism. The problem of incoherence is that a layer is too thick (on the order of 100 s of microns) for the reflected light at the boundaries to overlap and interfere with the original beam. This is a consequence of absolute normal incidence being an imperfect approximation. Any small deviation from perfect perpendicular incident light becomes a problem when the layer is too thick, as demonstrated in figure A1.

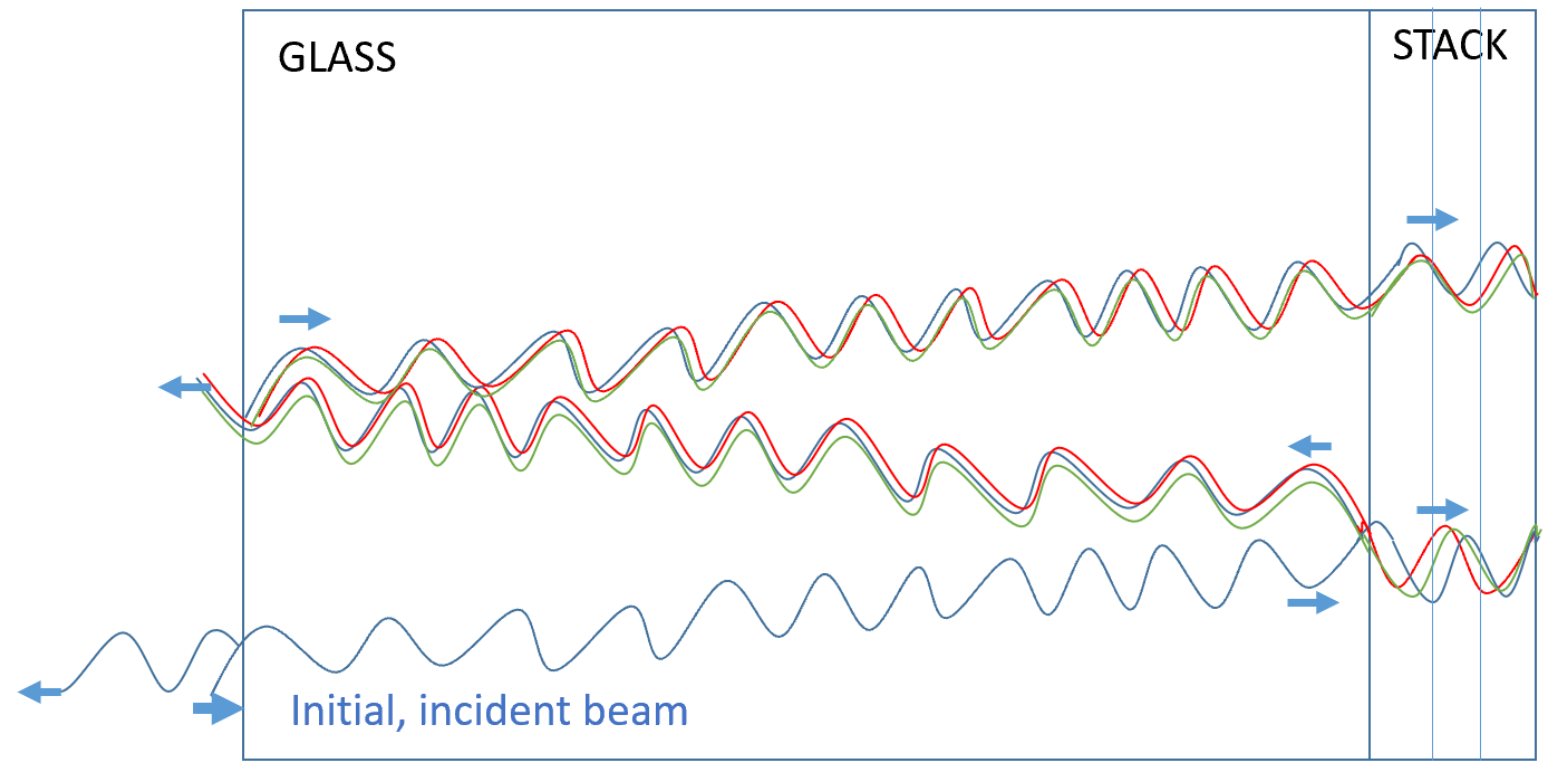

Figure A1. Visual demonstration of the incoherence problem.

In order to model this situation, forwards- and backwards-propagating beams that are the sum of all reflections in the incoherent layers need to be treated as intensities. This decouples this part of the light in the device from the phase-coherent light within the smaller layers. In other words, the matrices become identical to the original transfer matrix ones with the exception that reflectance and transmittance coefficients are used rather than Fresnel coefficients, and (for perfectly transparent incoherent layers) the phase factors are always unity, rather than a plane wave propagation.

If only one glass thick incoherent layer is involved, and is positioned in front of the coherent stack, a simple sum-series formalism can be used to link the contributions of the incoherent layer (and all its reflections) the light incident into the coherent stack, as following: 
Look at reflected power rather than reflection of E-fields:

$r \rightarrow|r|^{2}$ (or R) $\quad t \rightarrow n_{1} / n_{2} \cdot|t|^{2}$ (or T)

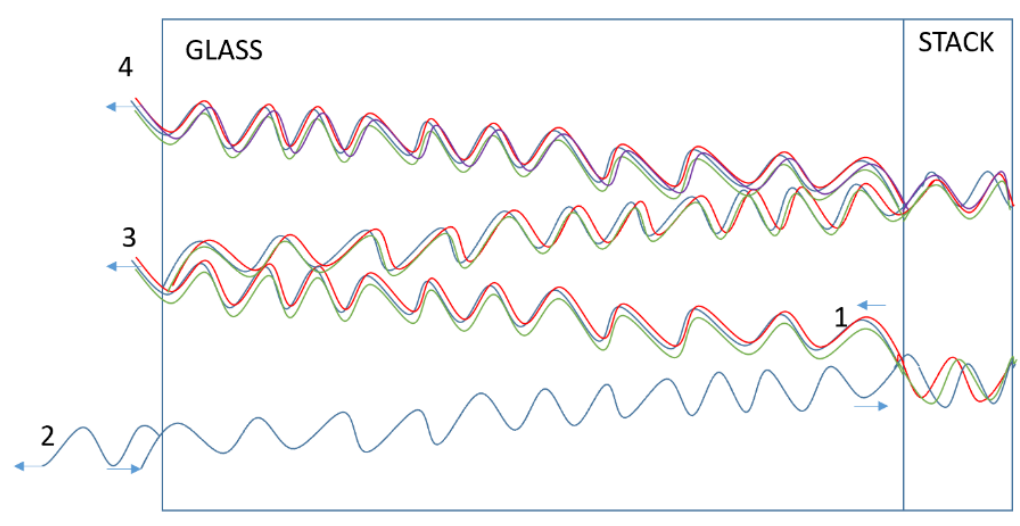

When adding the incoherent glass, $\mathrm{R}_{\text {stack }}(1)$ becomes:

$R_{\text {glass }}(2)$

$+\mathrm{T}_{\text {glass }} \cdot R_{\text {stack }} \cdot T_{\text {glass }}(3)$

$+\mathrm{T}_{\text {glass }} \cdot R_{\text {stack }} \cdot R_{\text {glass }} \cdot R_{\text {stack }} \cdot T_{\text {glass }}(4)$

$+.$.

Figure A2. Visual demonstration of the sum-series used to model incoherent layer.

$R_{\text {total }}=R_{\text {glass }}+T_{\text {glass }} \cdot R_{\text {stack }} \cdot T_{\text {-glass }}+T_{\text {glass }} \cdot R_{\text {stack }} \cdot R_{\text {glass }} \cdot R_{\text {stack }} \cdot T_{\text {-glass }}+\ldots$

$R_{\text {total }}=R_{\text {glass }}+T_{\text {glass }}^{2} \cdot \sum_{n=0}^{\infty} R_{\text {stack }}^{n+1} \cdot R_{\text {glass }}^{n}$

$R_{\text {total }}=R_{\text {glass }}+T_{\text {glass }}^{2} \cdot \frac{R_{\text {stack }}}{1-R_{\text {stack }} \cdot R_{\text {glass }}}$

This is for example the formalism implemented in the free open-source code of the McGehee group (https://web.stanford.edu/group/mcgehee/transfermatrix/)

In reality, however, there will be phase attenuation for glass if the light energy is higher than the so-called glass gap (starts around 350nm wavelength).

$R_{\text {total }}=R_{\text {glass }}+T_{\text {glass }} \cdot R_{\text {stack }} \cdot T_{- \text {glass }} \cdot e^{-\frac{4 \pi k}{\lambda} \cdot 2 t}+T_{\text {glass }} \cdot R_{\text {stack }} \cdot R_{\text {glass }} \cdot R_{\text {stack }} \cdot T_{- \text {glass }} \cdot e^{-\frac{4 \pi k}{\lambda} \cdot 4 t}$ $+\ldots$

In addition, if the position of the glass layer changes, or there are several incoherent layers, this approach becomes much more complicated. Once the incoherent light reaches back into the coherent part of the stack, coherent interference needs to be applied to this part of the light again, before it possibly couples into the incoherent layer again, etc.

This is why the approach in the work of full transfer matrix treatment with sub stacks that are coherent or incoherent, is much more efficient. In effect the device is divided into sub stacks which are coupled to each other in a formalism similar to the transfer matrix. In effect we bundle all the coherent matrices into one matrix, modify this matrix to denote intensity rather 
than electric field, and then repeat the transfer matrix procedure with the incoherent layers, treating the whole coherent stack as one layer. This is fully expounded upon in the work of Harbecke and shown in the Matlab implementation in flowchart 4A in Chapter 4. An implementation of this script has been provided open source ${ }^{103}$. 


\section{Appendix A2}

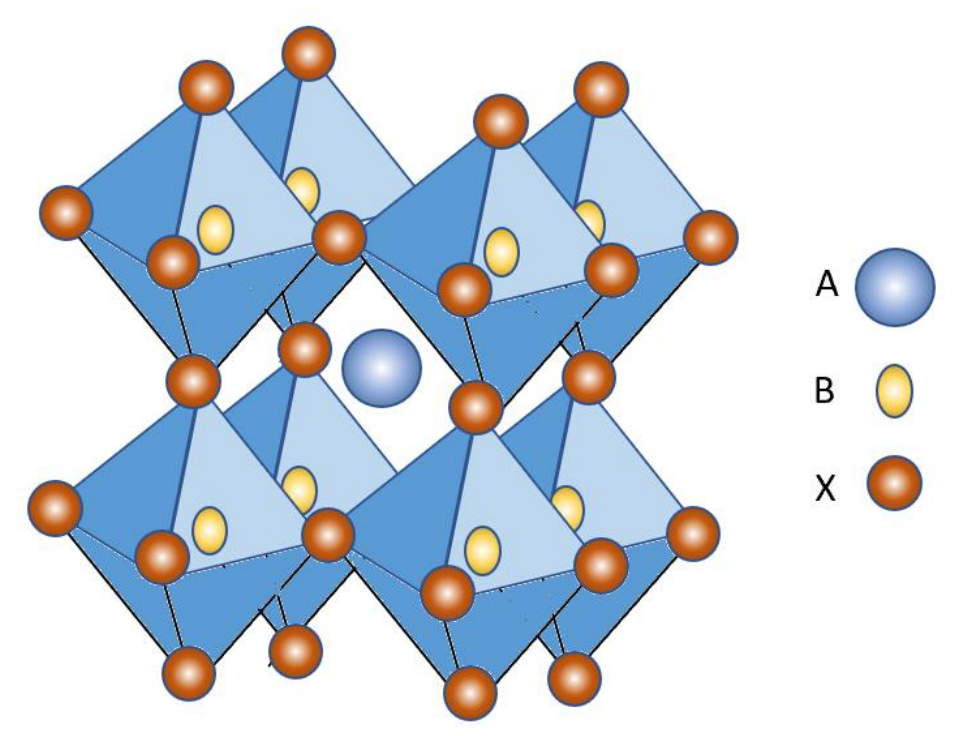

Figure A2.1. a standard perovskite crystal consisting, commonly ${ }^{106}$, of an organic cation, $\mathrm{A}=$ (methylammonium (MA) $\mathrm{CH}_{3} \mathrm{NH}_{3}{ }^{+}$; formamidinium (FA) $\left.\mathrm{CH}_{3}\left(\mathrm{NH}_{2}\right)_{2}{ }^{+}\right)$, ${ }^{3-5}$ a divalent metal, $\mathrm{B}=\left(\mathrm{Pb}^{2+} ; \mathrm{Sn}^{2+}\right.$; $\left.\mathrm{Ge}^{2+}\right), \frac{4.6}{6}$ and an anion $\mathrm{X}=\left(\mathrm{Cl}^{-} ; \mathrm{Br}^{-} ; \mathrm{I}^{-}, \mathrm{BF}_{4}^{-} ; \mathrm{PF}_{6}^{-} ; \mathrm{SCN}^{-}\right)$.

As briefly discussed in Section 4.2, there exist modifications of the perovskite solution to alter certain properties such as adhesiveness (in the case of AVA-MaPbI), but also efficiency and stability. An impactful paper on one such modification was published by Saliba et al ${ }^{106}$, demonstrating the increased stability and efficiency of perovskite consisting of a triple cation blend. The "standard" perovskite contains an organic cation of methylammonium (MA), a halide of lodide $(\mathrm{I})$ and a divalent metal of lead $(\mathrm{Pb})$, arranged in the structure outlined previously in chapter 2 and shown again in Figure 4.14. Replacing certain components in this unit cell can impact the stability of the crystal, as well as the bandgap of the useful "dark" perovskite $\alpha$-phase and the available other phases at room temperature. The main reason for these changes is the different effective ionic radii of the substituted elements, which impacts the Goldschmidt tolerance factor according to the effective ionic radii of the used elements ${ }^{107}$. This tolerance factor determines how stable the most useful crystal phase structure will be, as depicted in Figure 4.15. The other phases, such as the yellow $\delta$-phase, are not useful for either optical absorption or electrical conduction. In fact, the perovskite will always be in an equilibrium between the shown phases. This means that, with the optimal 
mixing of elements, an equilibrium can be reached, whereas much of the material as possible is in the useful $\alpha$-phase.

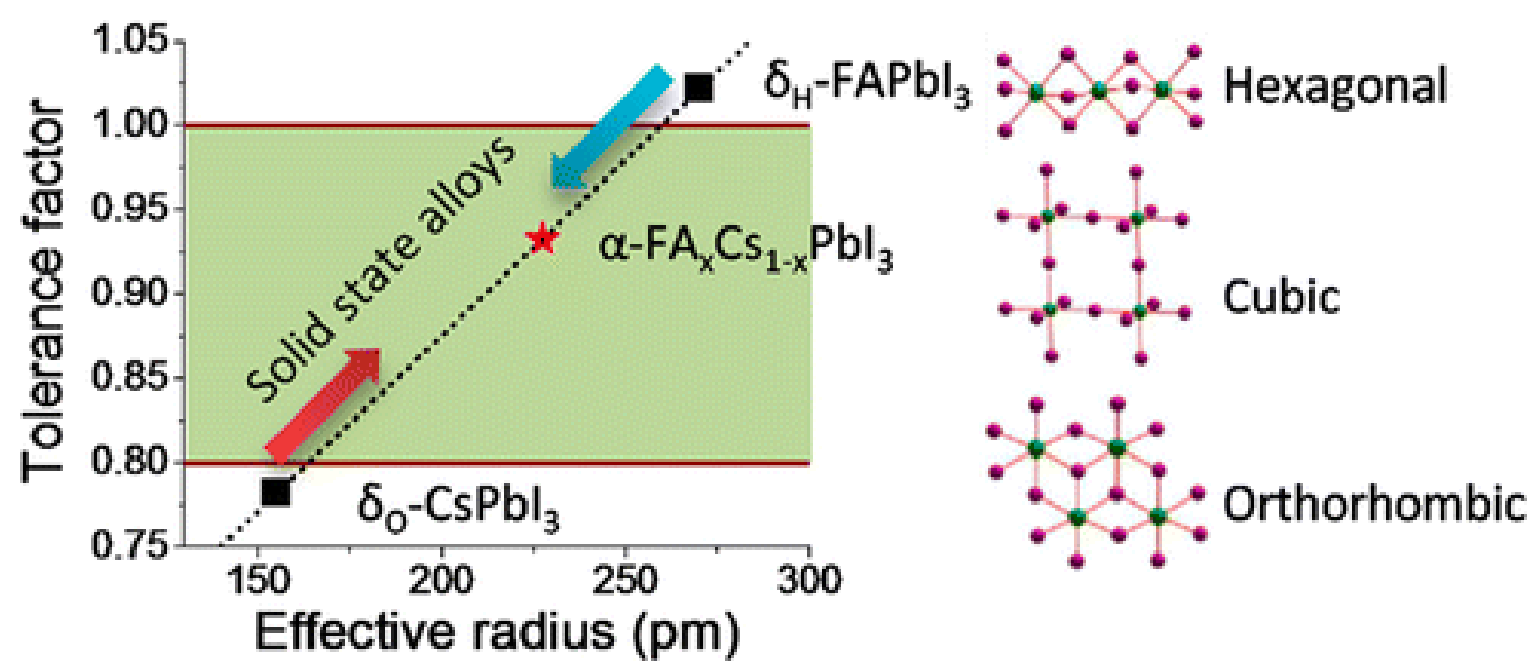

Figure 4.15. Depiction of the impact of molar ratios of components on the most stable crystal structure of the solid material at room temperature. Modified upon reproduction from ${ }^{107}$.

This optimal blend was determined experimentally by Saliba et $\mathrm{al}^{106}$, and contains three different cations, Cesium (Cs), Methylammonium (MA) and Formamidinium (FA), in an optimal molar ratio, empirically determined as $\mathrm{Cs}_{0.05}\left(\mathrm{MA}_{0.17} \mathrm{FA}_{0.83}\right)_{0.95} \mathrm{~Pb}\left(\mathrm{I}_{0.83} \mathrm{Br}_{0.17}\right)_{3}$, where the fractional indices stand for the molar proportions. The yield of this mixture managed to get efficiencies of up to $21.1 \%$, a record at the time, though work has been released since, getting efficiencies up to $25.2 \%{ }^{108}$.

The Saliba paper also discusses stability considerations, noting that humidity, as well as the glovebox atmosphere and even ambient temperature can have a significant impact on the device performance. This might be seen as one of the downsides of these devices, as the industrial fabrication process typically requires procedures that are easily reproducible and stable on a mass scale. Reproducibility is such a common issue for these triple cation devices that papers have been written with very precise recipes to address this exact issue ${ }^{90}$. It should be noted that in all these recipes, complex interlayers are key in gaining high efficiency, since interlayer recombination seems to be the main limitation ${ }^{86}$. 

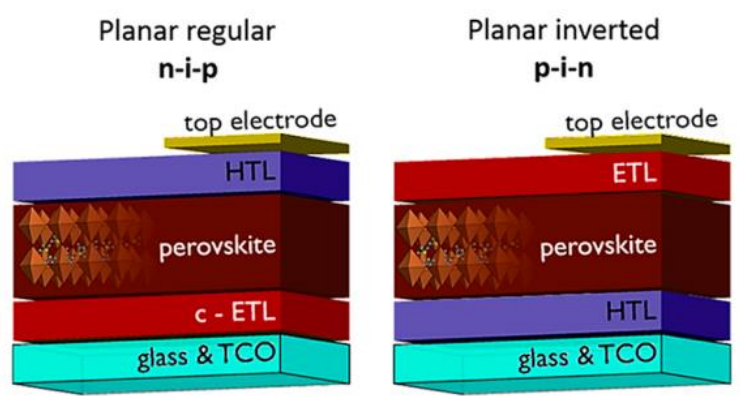

Figure 4.16 Architectures of the standard triple cation perovskite cell. Modified upon reproduction from ${ }^{90}$.

Nonetheless, cells using this altered type of perovskite have been shown to demonstrate record-high efficiencies, and as such significant efforts were undertaken to reproduce the architecture for the purpose of this thesis as well. However, even when following the recipes described in literature very carefully, cells failed to yield the same results. Initially, the PTAAPerov3C-C60-BCP-Cu inverted architecture was attempted. Many failed attempts to attain a reasonable efficiency, in addition with difficulties with the evaporator caused a switch to glass/FTO/c-TiO2/mp-TiO2/ CH3NH3Pbl3/spiro-MeOTAD/Au regular architecture, which is described in for example ${ }^{109}$. With this architecture personal top efficiencies of up to $6.9 \%$ were achieved, and these top devices represented a very small fraction of the many batches that were fabricated. Some IV curves of the fabricated devices are shown in Figure 4.17. Improvement of up to $10 \%$ could be attained by using a cleanroom-specific recipe for the triple perovskite, which had slightly different molar ratios, and which was developed through much trial and error by perovskite users there (who managed to attain efficiencies up to $18 \%$ ). However, this is still a far cry from reported values in literature. 


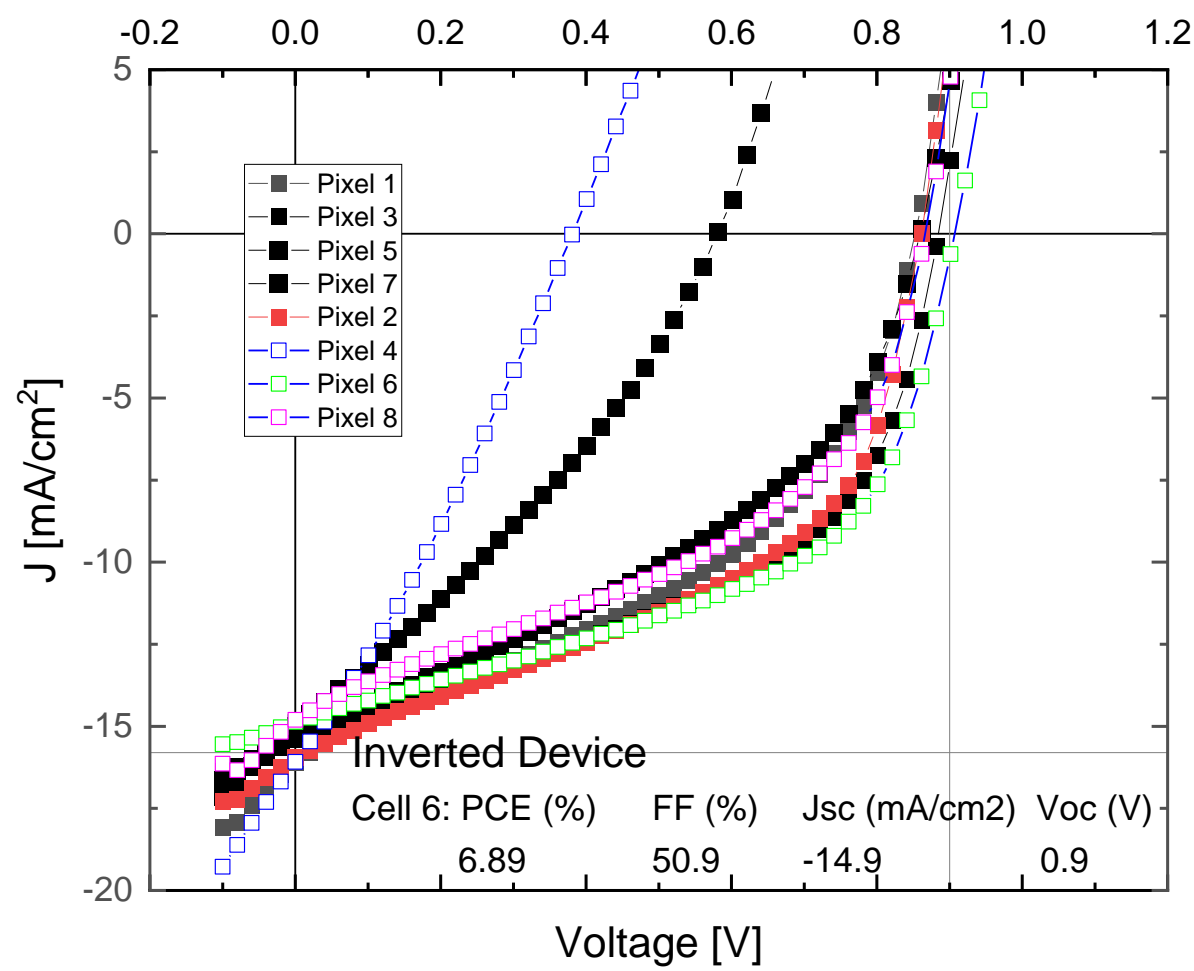

Figure 4.17. IV curves for one of the fabricated inverted triple cation devices, using PTAA architecture as described by Stolterfoht et $\mathrm{al}^{90}$.

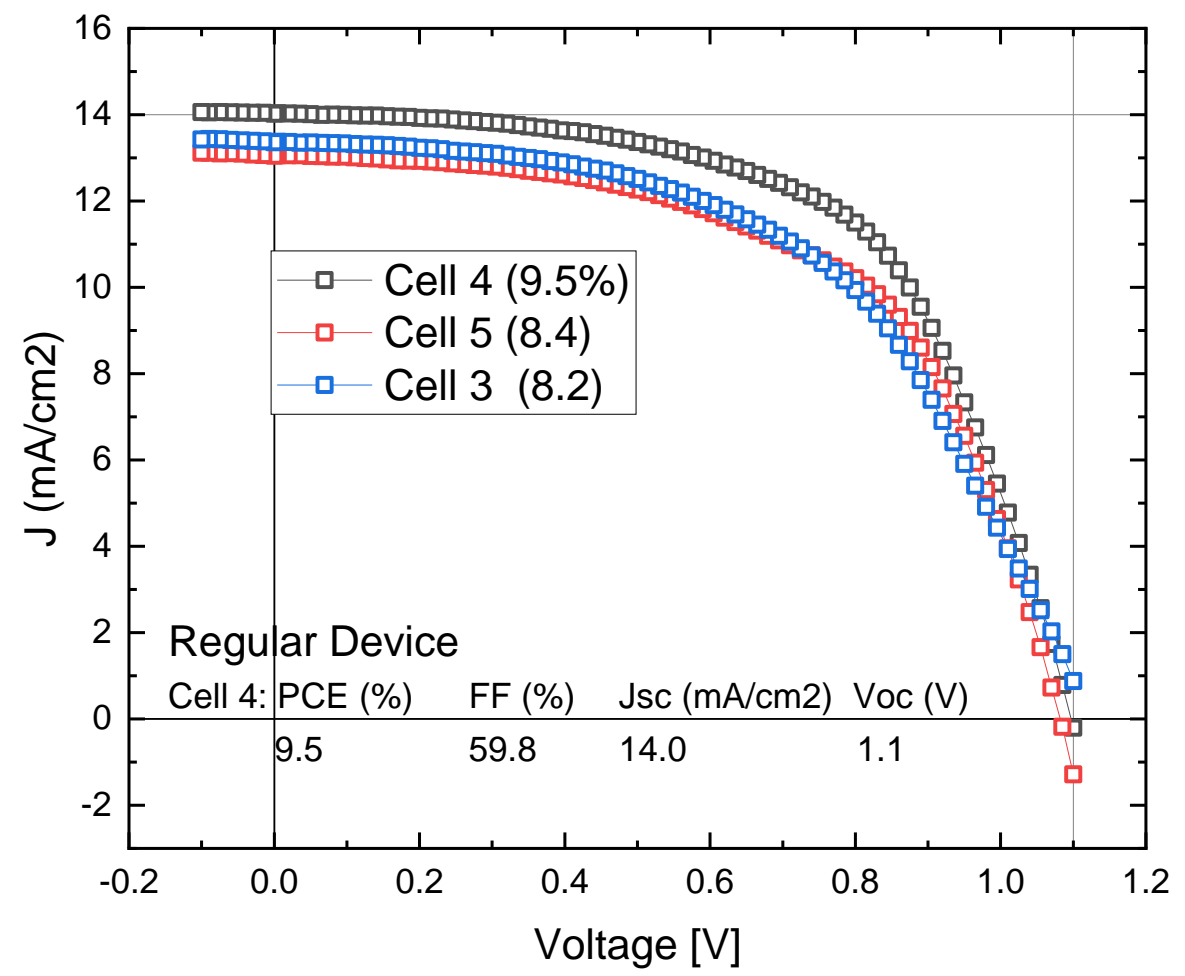

Figure 4.18 $\mathrm{JV}$ curves for one of the fabricated regular triple cation devices, using Pedot Au architecture with local cleanroom recipe 
Key factors in making perovskite so difficult to reproduce are the precise required ratios of the multiple components, the order in which the components can be added together, the stability of the solution, the glovebox ambient temperature and atmosphere as mentioned before $^{63}$, as well as the method of deposition of the perovskite solution. The used method for our work was spin-drop deposition ${ }^{110}$, which involves first depositing the perovskite solution on a sample, then initiating a carefully tailored spin-speed program on a spin-coater holding the sample, and depositing the antisolvent (there are various kinds with various reported effects) at the correct time under the correct angle to sublimate the crystalline, "darker" phase of the perovskite. Failure to replicate any of these aspects accurately can result in the wrong phase of perovskite, pinholes, or unstable crystallization. After deposition the layer is annealed for on a hot-plate of 100 degrees Celsius for 40-60 minutes. Again, conditions of the box can have an effect. All this has given perovskite a reputation of being notoriously difficult to reproduce.

In addition, there are certain other aspects which are still not fully understood for the material, such as the so-called light soaking effect which makes the perovskite devices more or less efficient after exposing them to AM1.5 lamp light. The most common explanation for this is the fact that the interstitial ions in the perovskite crystal ( $A$ in Figure 4.14) may in fact be mobile, even in the solid phase, and will accumulate at interfaces depending on the applied voltages and light to which the material is exposed ${ }^{32}$. This implies that even during fabrication, even the lighting of the perovskite in the glovebox may affect the optimal crystallization, which would be another explanation for the variety in cleanroom recipes across research groups.

While these difficulties at reproducing efficiencies may seem troubling and cast doubt on the industrial application of perovskite, there are many groups that have indeed succeeded at creating ever-higher efficiencies with perovskite devices, using special perovskite structures such as a $2 \mathrm{D} / 3 \mathrm{D}$ ordering of the crystal ${ }^{111}$, and even the addition of caffeine ${ }^{112}$. For this reason, it remains a subject of enthusiastic research in the academic world. 